

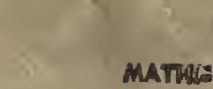

STAT.

UBRARY
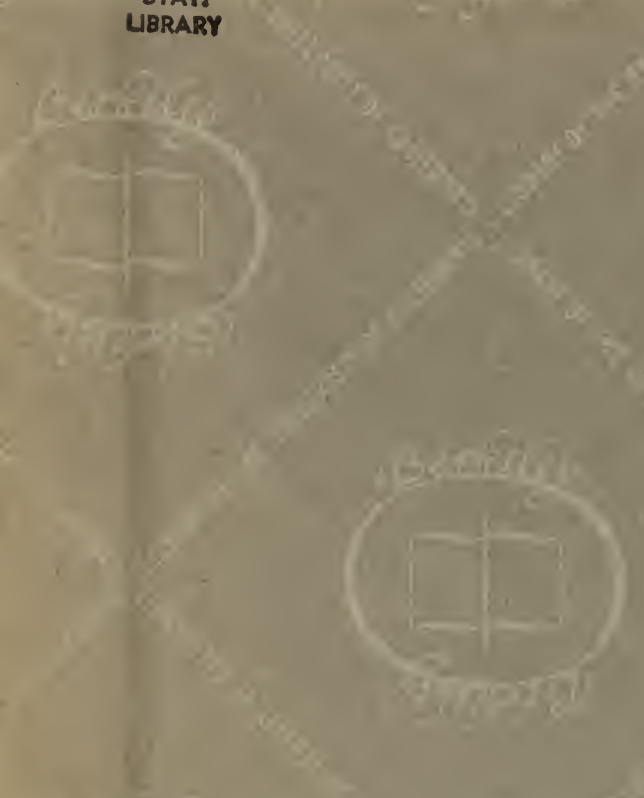

$2020 \%$
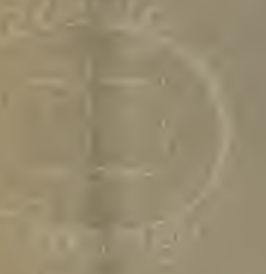

.
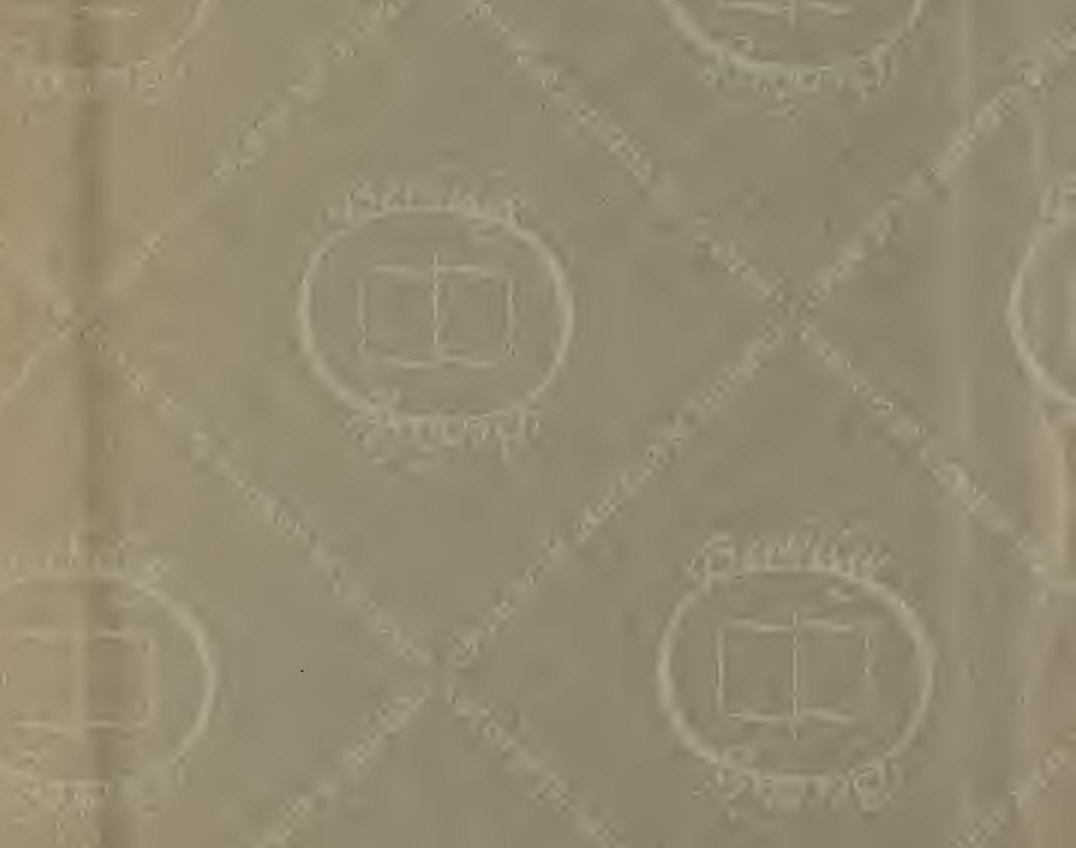


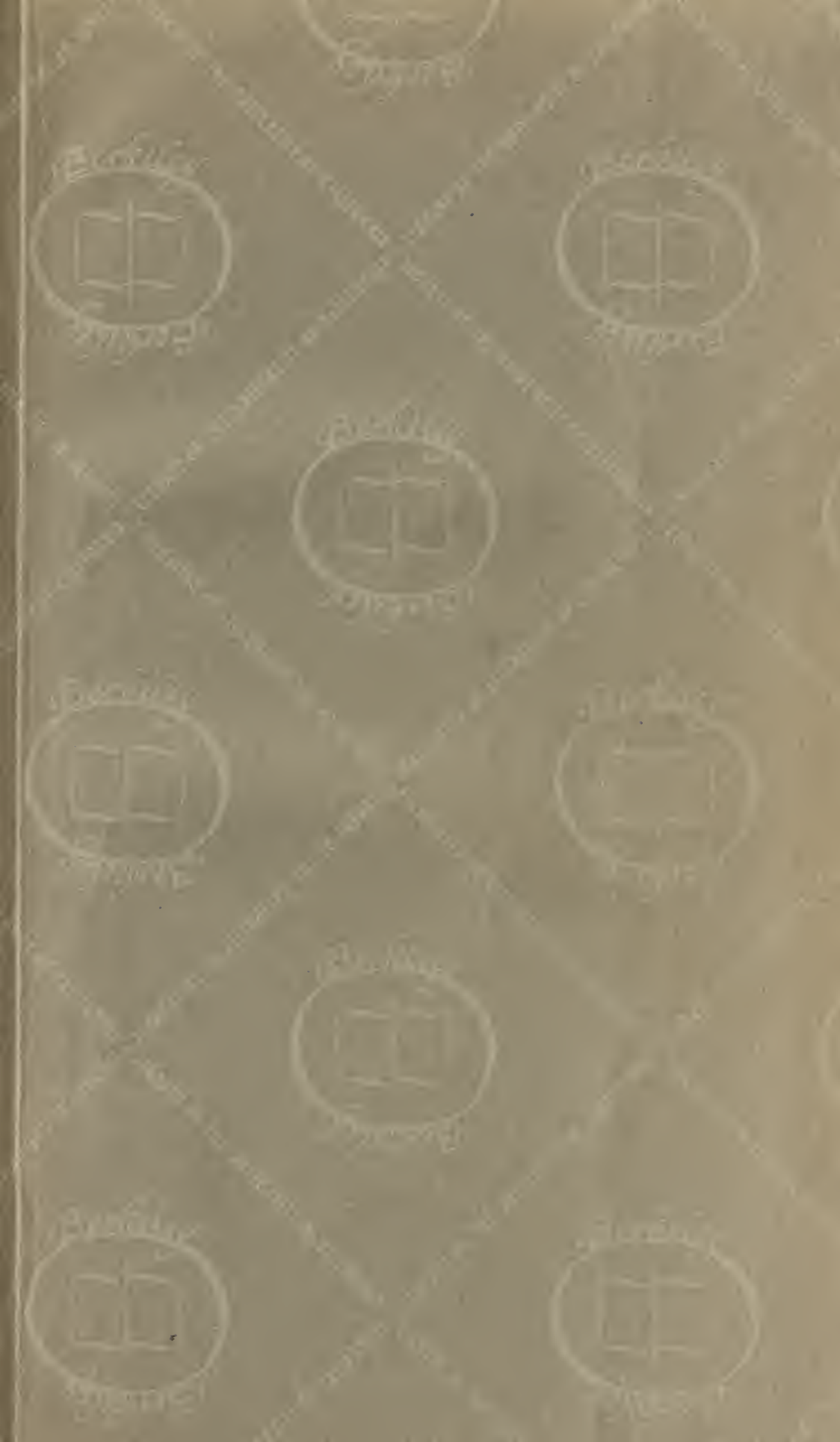







\section{ANALYTICAL VIEW}

\section{SIR ISAAC NEWTON'S}

\section{PRINCIPIA.}

BY

HENRY LORD BROUGHAM, F.R.S.

MEMBER OF THE NATIONAL INSTITUTE OF FRANCE, AND OF THE ROYAL ACADEMY OF NAPLES;

AXD

E. J. ROUTH, B.A.

FELLOW OF ST. PETER'S COLLEGE, CAMBRIDGE.

\section{LONDON :}

LONGMAN, BROWN, GREEN, AND LONGMANS; C. KNIGHT EDINBURGH : A. AND C. BLACK; GLASGOW : R. GRIFFIN. 1855. 


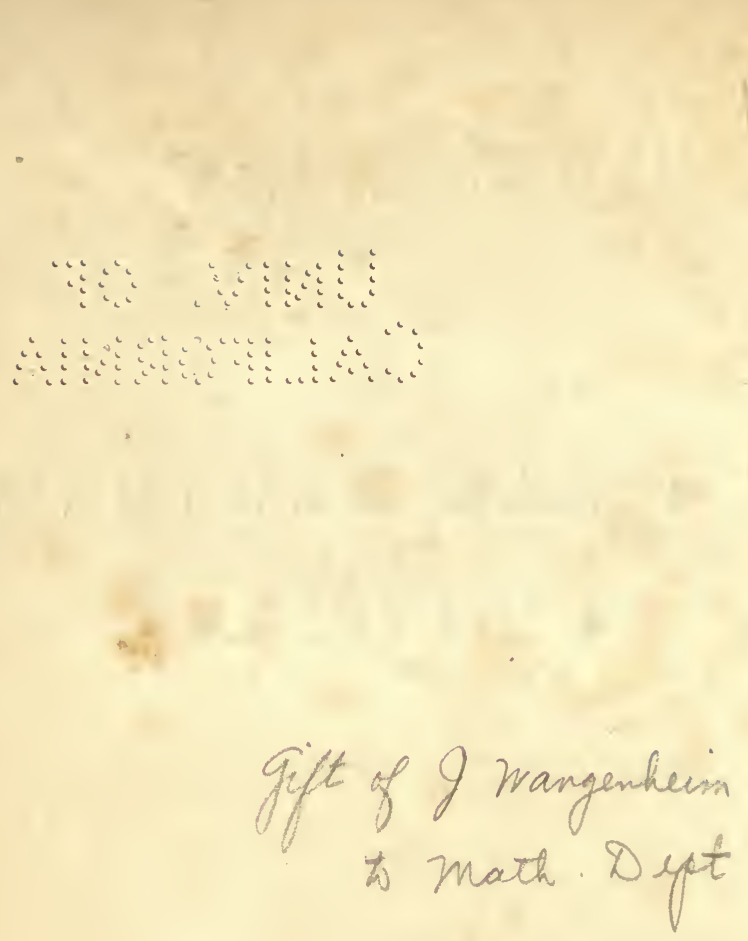


TO

\section{PROFESSOR THE BARON PLANA, OF TURIN,}

THIS WORK IS INSCRIBED,

AS A SMALL TOKEN OF RESPECT FOR THE GREAT TALENTS AND PROFOUNO LEARNING OF THAT EMINENT MATHEMATICIAN. 



\section{CONTENTS.}

General remarks. - Division of the work into Three Books, 1. State of Physical Astronomy and Dynamics before Sir Isaac Newton, 2. - General law of gradual discovery, ib. - Examples - Logarithms, ib. - Fluxions, ib. -- History of this Calculus, 3. - Calculus of Variations, 5. - Euler, Lagrange, Bernouilli, Emerson, 7. - Copernican Theory, ib. - Galileo's discoveries, 8. - Kepler's laws, ib. - Huygens, ib. - Borelli, ib. - Hooke, ib. - Halley, ib. - Peculiar maturity of the Newtonian theory as at first delivered, 10. - Nothing since supplied to its demonstration which Sir Isaac Newton originally had left imperfect, $i b$. note. - Three services beside the discovery of Gravitation, performed by this work to science, $i b$. Prodigious merit, even if gravitation were struck out of it, 11. - Reception of the Principia slow even in England, ib. Editions, ib. - Maclaurin and Voltaire, 12. - Difficulty of reading it from its Conciseness and Synthetical form, ib. - Jesuits' edition, 13. - Submission to papal authority, ib. - Pius VII.'s liberality, Sorbonne and Buffon, ib. note.

\section{I.}

Definitions of the Principia, 14. - Two remarks on them, ib. Early view given of the Great Discovery to which the whole work leads, 15. - Three laws of motion, ib. - Six corollaries

wo them, ib. - Summary of dynamics, as it existed before Sir Isaac Newton, 16. - Scholium to the laws of motion, upon uniform and accelerated motion, 17. - Laws and formulas on velocity, space, and time, $i b$. note.

(Secrion I. Principia.) - Method of prime and ultimate ratios, 19. - Treatise on Fluxions, 20. - Fundamental principle of the generation of quantities, ib. - Generation of curves, 21. - 
Nomenclature, 22. - Notation, ib. - Advantages and disadvantages of the two notations, 23. - Finding fluxion (or differential) of a rectangle, 24. - Square, $i b$. - Solid, $i b$. - Quantity of any power by analogy, $i b$. - Deduction of the rules from other principles, 25. - Finding fluents (or integrals), ib. Method of drawing tangents, 26. - Normals, ib. - Exemplified in the conic sections, 27. - Problems of maxima and minima, ib. - Example, ib. - Quadrature of curves, 28. - Example: Parabola, $i b$. - Rectification of curves, $i b$. - Example: Circular arcs, ib. - Measurement of solids, 29. - Example : Cone, sphere, and cylinder, $i b$. - Finding radius of curvature, $i b$. Example: Parabola, 30. - Addition of constant quantity in integration, $i b$. - Method of investigation used by Sir Isaac Newton, $i b$.

Subjects of the Three Books, 31.

(Sectron II. Principia.) - Areas proportional to the times, round a centre of forces, $i b$. - Empirical discovery of Kepler, $i b .-$ Proposition and its converse proved, 32. - Corollaries to this fundamental law of centripetal forces, 33. - Law of circular motion, the force as the square of the arc, and inversely as the distance, 34. - Demonstration, ib. - Importance of this proposition, 35. - Consequences in showing the laws of motion, $i b$. Demonstrates the general law, of which Kepler's rule of the sesquiplicate ratio is one case, 36. - Demonstrates the law of the inverse square of the distance, 37. - Law extended to other curves, $i b$. - Consequence that bodies fall through portions of the diameter, proportional to the squares of the times in which they describe the corresponding arcs, 38. - Moon being deflected from the tangent of her orbit by gravitation proved from hence, 40. - Reference to other proofs of it, 41. note. - Investigation of General Expressions for Centripetal Force, 42. - Five formulas given, 43. - Herrman's, 44. - Laplace's, 46. - Maclaurin's, ib. - J. Bernouilli's, ib. - Proof that this is taken from Prop. VI. B. I., Principia, 47. - Keill's imperfect acquaintance with this subject, ib. - Herrman's mistake, 48. - Formulæ exemplified in the case of the parabola, 49. - Ellipse and hyperbola, $i b$. - Centrifugal forces. - Formulæ of Huygens, 50. Subject of Centripetal forces divided into four heads, ib. - $\mathrm{i}$. The force required to describe given conic sections. - ii. The drawing conic sections from points or tangents being given; 1 . When one focus is given; 2. When neither is given. - iii. The finding the motion in trajectories that are given.-iv. The finding trajectories generally when the forces are given.

i. 'The first head is treated of in the remainder of the Second, and the whole of the Third Sections of the Principia. Central force in a circle, when the centre of forces is the centre of the circle, or any other point in the diameter, or in the circumference re- 
spectively, 51. - Central force in an ellipse when the centre of force is the centre of the ellipse, 53. - Converse of the proposition, 54. - Equality of periodic times in concentric similar curves, when the law of the force is as the distance, $i b$. - Consequence of the sun being in the centre of the system, 55 .

(Section III. Principia.) - Law of forces when the centre of forces is in the focus of the curve, ib. - General theorem that in each of the three conic sections the law is the inverse square of the distance, $i b$. - Converse of the proposition proved, 57. - J.Bernouilli's objection to Sir Isaac Newton's proof, 58. - Shown to be groundless, $i b$. - His objection to Herrman's demonstration, 59. - Refuted, $i l$. - Motion in concentric conic sections, the centre of forces being in the focus, $i b$. - Demoivre's theorem, 60. - Demonstration of Kepler's law of sesquiplicate ratio generally, 61. - Inverse problem of finding the orbit from the force being given, $i b$. - Determination of the nature of the orbit from the forces, 62. - Sir Isaac Newton's observations on the investigation of disturbing forces, $i b$. - Anticipates Lagrange's investigation, 63. note. - Importance of Perpendicular to the Tangent and Radius of Curvature in all these inquiries, 63.

i. - (Secrions IV. V. Principia.) - General observations on these sections, 64. - Illustration of their use in Physical Astronomy, 65. - Further illustration from their application to the problems on comets, ib. - Comparison of theory with observation by Newton, 66. - By Halley, 67. - Comets of 1680, 1665, 1682, 1683, ib. - General remarks on the importance of these sections, 68.

iii. Motion (1) in given conic sections, (2) in straight lines, ascending or descending.

iii. - (1.) - (Section VI. Principia.) - Method of determining the place of a body in a given trajectory, being a conic section, at any given time, 69. - Solution for the parabola, 70. - Method conversely of finding the time, the places being given, 71. Solution for the ellipse, or Kepler's problem, ib. - Difficulty of the problem, 72. - Sir Isaac Newton's proof that no oval is quadrable, ib. - Class of curves returning into themselves and quadrable, beside the class mentioned by him of ovals connected with infinite branches, 73. - Demonstration respecting the ellipse, 74. - Observations, ib. - Sir Isaac Newton's solution of Kepler's problem indirectly by the cycloidal, $i b$. - Another solution directly by a cycloidal curve, 75. - Astronomical Nomenclature, 76 .

ii - (2.) - (Section VII. Principia. - Motion ascending and descending in straight lines, $i b$. - Determination of times of descent and ascent, $i b$. - Determination of velocities in case of parabolic lines, 77. - Time of moon falling to the eartli, 79. - 
Analogy of the case of planets falling into the sun, to the structure of bees' cells, $i b$. note. - General solution of the problem for all kinds of centripetal force and orbit, 79 .

iv. - (Section VIII. Principia.) - Observations upon the general inverse problem of centripetal forces, or finding the orbit, the force being given, 80. - Sir Isaac Newton's solution, though geometrical, is less synthetical than usual, 82. - Determination of the trajectory generally by the method of quadratures, $i b .-$ Remarks on that method, 85. - The subject illustrated in the case of the inverse cube of the distance, $i b$. - Another solution given by a polar equation, 86. - Conclusion of the subject of centripetal forces in fixed orbits, and round an immoveable centre, $i b$.

Of motion in moveable orbits divided into two heads, 87.- i. When the orbit and centre are in the same plane. - ii. When the orbit's plane is eccentric.

i. - (Section IX. Principia.) - Determination of the motion of the apsides, ib. - Proportion of force to distance, which make the axis or apsides advance and retire respectively, 88. - Determination of motion of apsides from the force and conversely, 89. - Gravitation the only force by which the line of apsides can coincide with the fixed axis, 90. - Motion of the apsides with different centripetal forces, $i b .-$ Application of the theory to the motion of the moon's apsides, 91. - To the motion of the earth's apsides, 92. - Sir Isaac Newton did not reconcile the theory with observation, as regards the moon, $i b$. - Misstatement of Bailly on this subject, $i b$. - History of the question respecting the agreement of the theory with the observation, 93. - Euler, D'Alembert, Clairaut, ib. - Clairaut's error, and his discovery of the agreement between the theory and fact, 94. - Laplace's solution and discoveries, $i b$. - Reference to the papers of the three mathematicians on the problem of these bodies, $i b$. note. - Bailly's further erroneous statement respecting Sir Isaac Newton, 95. - Proof of that error, ib. - General opinion of Bailly on the Newtonian lunar theory erroneous, 96 . - Testimony of Laplace, 97. - Error of Laplace respecting Sir Isaac Newton's assumption as to the perigeal motion, $i b$.

ii.-(Secrron X. Principia.) Determination of trajectnries in a given plane, when the centre is out of that plane, 98. - Of trajectories on a curve surface, 100. - Example of the circle and cylinder, ib. - Motion of pendulums, 101. - Properties of hypercycloids, and hypocycloids, ib. - Isochronism of the cycloid, 102. - General solution for all curves by the evolutes, $i b$. - Peculiarity of cycloid and logarithmic spiral in being their own evolutes, 103. - Reason why Sir Isaac Newton took the case of hypercycloids and hypocycloids, and not cycloids, $i b$ - Measurement of gravity by the pendulum, deduced from these propositions, $i b$. - Conclusion 
of the subject of motion where the centre of forces is immoveable, ib.

(Secrion XI. Principia.) Motion in orbits where the centre is disturbed, or where other forces disturb the motion - divided into three heads,

i. Disturbance produced by the mutual action of two bodies revolving round one another, 104. - Demonstration of their motion round each other, and round the common centre of gravity, 105. - Motion referred to a body in the centre of gravity, 107. - Amount of the body which must be in the immoveable centre that it may act there, as the bodies would act on each other, were one to be in the fixed centre, $i b$. - Determination of their absolute trajectories in space, $i b$. - Application to the earth and moon, 108.

ii. Disturbances produced by the action of the whole bodies of any subordinate system on each other, and by the bodies of other systems on any given subordinate system, illustrated from Laplace, $i b$. - Remarks on Newton's investigations, and the problem of three or more bodies, 109. - Comparative disadvantages under which he laboured, $i b$. - Improvement, first, of the calculus itself, and secondly, by the introduction of that of variations, peculiarly fitted to facilitate these inquiries, 110. - How the latter especially bears on the subject, $i b$. - Motion of the moon's apsides and nodes, 112. - Variation in the rate of both their motions, ib. - Acceleration of the moon's motion, $i b$. - The cause discovered by Laplace from the algebraical expression, 113. Connexion between the transverse axis and the mean motion, $i b$. - Kepler's law demonstrated, 114. - Proved by the mere examination of the algebraical expression only to be true if there are no disturbing forces in action, 115. - Same inspection likewise shows the retardation of the apsides and nodes to be caused like the moon's acceleration by the decrease of the earth's eccentricity, ib. - Confirmation of the calculus by actual observation, $i b$. - Slow secular inequality of the moon discovered by Laplace, in diminution of her secular acceleration, 116. - Irregularity of other orbits and motions, $i b$. - Motion of earth's apsides produced by the disturbing forces of the greater planets, 117. - Variation of orbits of other planets, $i b$. - Disturbances at first seem not reducible to any fixed rule, 118. - Euler's attempt and errors, ib. - His important discovery, $i b$. - Discovery by Lagrange and Laplace of the stability of the system, and universal operation of the rule, 118. - Mean motions of Jupiter and Saturn commensurable, 119. - Proportion of motion and distances of Jupiter's satellites, $i b$. — Laplace's remarks on Jupiter and Saturn, ib. No satellite but the moon disturbs its primary, 120.- The greater axes of the planetary orbits do not vary from one long period to another, 121. - The period of their change being 
short, the mean motions of the planets undergo no secular variation, 122. - General law of stability of the system, 123. - General reflexion, 124. - No resistance of an ethereal medium, nor any transmission of gravity in time, $i b$.

iii. Marvellous powers of Sir Isaac Newton in discussing the subject of disturbing forces, 125. - Great superiority to all his successors, ib. - Determination of the disturbances arising from a third body's action upon other two, and theirs upon the third and each other, - or problem of three bodies, 126. - Attraction as the distance, alone preserves all motion undisturbed, $i b$. - Produces immense velocities, 127. - The small actual derangement shows the inverse square of the distance not to be much departed from, 128. - Investigation of the general problem, $i b$. - Case of moving bodies and proportion of masses to forces, 131. - Accelerations and retardations at different parts of the orbit: quadrature and syzygies, $i b$. - Different planetary variations deduced by Sir Isaac Newton from the solution, 132. - Extraordinary generalization of the problem to precession and tides, $i b$. - Sixty-sixth proposition and its corollaries embrace all that has been done on the subject, 134. - Error of Laplace, ib. note.

Attraction under two heads, i. that of spherical; ii. that of nonspherical bodies.

i. (Section XII. Principia.)-Attraction of spherical snrfaces, 135. - Remarkable inferences showing the solidity of the earth, 136. - Attraction of spheres on particles beyond their surface, 137. - On particles within their surface, 139. - Five general theorems, $i b$. - Corollary comparing corpuscular attraction with centripetal forces, 140. - Peculiarity of the actual law of gravitation, ib.-General solution for all other laws of attraction, 141.Reduced to the quadrature of a curvilinear area, $i b$. - Solution of this quadrature, 142. - Remarkable result when the force is inversely as the cube, or any higher power of the distance, 144 . - Attraction of spherical segments, $i b$.

ii. (Section XIII. Principia.) - Attraction of bodies not spherical, 145. - Proportion of attraction to homologous sides of similar bodies, 146. - General theorem for attraction of all bodies as related to the centre of gravity, the force being gravitation, 147. - Attraction according to any power of the distance in any symmetrical solids, $i b$. - General solution, 148. - Laplace's formula for attraction, 149 .

Motions of infinitely small bodies like light, 150. - (SECtion XIV. Principia.) - Proportion of angles of incidence, refraction and reflection, $i b$. - Inflection and deflection, 151. - Subsequent experiments on the coloured fringes by flexion, $i b$. - General remark on the perfection of Newton's discoveries, 152.- Solution of Descartes' focal problem, $i b_{0}$ - Newton's optics, $i b_{0}$ - Dates 
of the publication of Lectiones Opticæ, Principia, and Optics, ib. note.

General conclusions from the Newtonian discoveries relating to attraction, 153. - Universal prevalence of gravitation, ib. Further proof of this from Herschel's discoveries in double stars, 154. - Those observed by Cassini, $i b$. - Not understood till the elder Herschel's time, $i b$. - Their revolutions round each other and periods, 155. - Apparently follow the law of sesquiplicate proportion, $i b$. note.

Three other important applications of the Newtonian theory of attraction, $i b$.

1. To find the weights of bodies at the sun and different planets, $i b$.

2. To find the masses of the heavenly bodies, and their densities, 156. - Singular confirmation of the Newtonian theory by Laplace's calculation on Jupiter derived from different sources, 157 .

3. Application of the theory to the figures of the heavenly bodies. $i b$. - The earth's figure as determined by Newton, ib. - Of Jupiter, compared with observations, 158. - Measurements and pendulum experiments show the earth not to be homogeneous, ib. - Newton's computation respecting the earth's figure, on the supposition of its being homogeneous, 159. - His fraction for the excess of the quotient diameter, $\frac{1}{2} 30$, still used as accurate, $i b$. - Remarks upon the wonderful completeness of the theory at its first establishment deduced from hence, $i b .-$ No improvements whatever upon that theory since his time, nor any defect found in its proofs, 160. - Note on structure of the Earth, 161.

II.

Introductory remark, 162. - Resistance of media in all motions except those of the heavenly bodies, $i b$. - Resistance of air, $i b$. Water, $i b$. - Pressure and motion of fluids, ib. - Hydrostatics, Hydraulics, Pneumatics, ib.

Arrangement of the Second Book of the Principia under five heads.

I. Motion of bodies in media which resist in different proportions to the velocity.

i. Where the resistance is as the velocity. (Section I. Principia.)

ii. Where the resistance is as the square of the velocity. (Section II. Principia.)

iii. Where the resistance is partly in one proportion to the velocity, partly in another. (Section I11. Principia.)

II. Spiral motion in resisting media. (Section $I V$. Principia.)

III. Motion of pendulums in resisting media. (Section $V$. Principia.) 
IV. Motion of projectiles in resisting media. (Section VII. Principia, in part.)

V. Pressure and motion of fluids.

i. Statics of fluids, or hydrostatics. (Section V. Principia.)

ii. Motion of fluids, or hydraulics. (Section VII. Principia, in part.)

iii. Motion propagated through fluids, whether elastic or nonelastic, including the pulses and waves, or acoustics. (Section VIII. Principia.)

iv. Circular motion of fluids, or vortices. (Section IX. Principia.)

Two general remarks on Book II., 163. - Much done before Newton, $i b$. - Summary of former discoveries, ib. - Archimedes, ib. - Galileo, ib. - Pascal, ib. - Torricelli, 164. - Much left to succeeding inquirers, $i b$. - Bernouilli, $i b$. - Clairaut, $i b$. Laplace, 165. - Value of this portion still great, and only less than that of the rest of the Principia, $i b$.

\section{ANALYTICAL VIEW. \\ PRINCIPIA.-BOOK SECOND.}

\section{CHAP'TER I.}

THE ELEMENTARY PRINCIPLES OF HYDROSTATICS, AND THE LAWS OF DENSITY OF AN ELASTIC FLUID COLLECTED ROUND A CENTRE OF FORCE.

I. Elementary Principles of Hydrostatics $\quad-\quad 167$ 1. What a fluid is, the terms viscosity, solidity, \&c., - $i b$.

2. What the foundation is on which the theory of Hydrostatics is built. Newton, xIx. - - $\quad-168$

3. The fundamental equation by which we know the properties of a fluid in equilibrium. Note I. - - 315

4. Three consequences of this equation. Note I. - $i b$.

(1.) That there must in all cases be a certain relation among the forces - $\quad-\quad-\quad-\quad i b$.

(2.) Level surfaces are surfaces of equal density $\quad-318$

(3.) Level surfaces are surfaces of equal temperature - 319

5. Newton, xx., fluids under the action of gravity only $\quad-170$

II. The Law of Density in a compressible Fluid under the action of a central force $\quad-\quad+\quad \quad-\quad \quad-170$ 
1. Solution of the question; defect in the application to physical questions. Newton, xxı. xxı. \&c. - $\quad 172$

2. First application - measurements of heights. Note II. 320 Second application - form of our atmosphere, the zodiacal light not part of the sun's atmosphere. Note II. -

322

\section{CHAPTER II.}

\section{THE FIGURE OF THE EARTH.}

1. Newton's calculation of the ellipticity of the earth; its defects - - - - - - -

2. An accurate investigation of the ellipticity on the supposition that the earth is homogeneous; the form thus found proved to be stable - $\quad$ - $\quad$ - $\quad$ - 178 Note III. - $\quad$ - $\quad$ - $\quad$ - $\quad$ - $\quad$ - 324

3. Newton's calculation of the law of variation of gravity $\quad-179$

4. Newton's application of his theory to the planet Jupiter - 181

5. The figure of the earth considered as heterogeneous, Clairaut and Laplace's results. Note IV. - $\quad-328$

a. The form of the strata - $\quad$ - $\quad-\quad \quad-328$

$\beta$. The law of variation of gravity - $\quad-\quad \quad-329$

6. The law of density in the interior of the earth. Note IV. 330

7. Whether the interior of the earth is solid or fluid. Note IV. 333

8. Measures to determine by observation the ellipticity of the earth's surface. Note IV. $\quad$ - $\quad$ - $\quad-337$

a. Measurement of degrees - $\quad$ - $\quad$ - $\quad 337$

f. Observations on the pendulum - $\quad-\quad \quad-338$

$\gamma$. Astronomical observations $\quad$ - $\quad$ - $\quad ~-~ 3411$

\section{CHAPTER III.}

THE MOTION OF A PARTICLE IN A RESistiNg MEDIUM.

1. The object and mode of conducting the inquiry - $\quad 183$

2. When the resistance varies as the velocity - $\quad 186$

a. Rectilinear motion - $\quad$ - $\quad$ - $\quad$ - $i b$.

$\beta$. Curvilinear motion. Section I. - - $\quad-\quad i b$.

3. When the resistance varies as the square of the velocity - 196

a. Rectilinear motion - - - $\quad i b$.

$\beta$. Curvilinear motion. Section II. - - $\quad-\quad i b$.

4. When the resistance varies partly as the velocity and partly as the square of the velocity, and the motion is rectilinear. Section III. - -

5. When the resistance varies as any power of the velocity and the motion is rectilinear - consideration of an analytical difficulty in the solution - the terminal

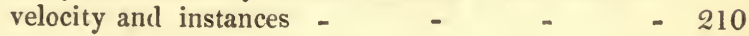


6. The motion of a particle in a resisting medium round a

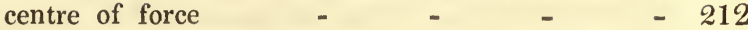

a. The method used by Newton - $\quad-\quad$ - $i b$.

$\beta$. The method supplied by the Planetary Theory.

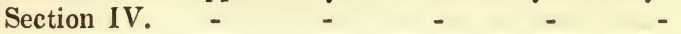

\section{CHAPTER IV.}

THE MOTION OF FLUIDS, AND THE RESISTANCE TO BODIES MOVING IN THEM. SECTION VIr.

1. Newton's investigation of the law of resistance to similar bodies

2. The manner in which the resistance depends on the form. of the body. Newton, xxxiv. and xxxv. - $\quad-226$

3. Their resistance when the body is a surface of revolution

4. The surface of least resistance, its properties and form Scholium, Prop. xxxiv. - $\quad-\quad-{ }_{-}-$

5. The law of resistance deduced from experiment. Prop. xL. and Scholium

NOTE V.-The Resistance Made to Bodies Moving in FLUIDS DEDUCED FROM THE GENERAL PRINCIPLES OF DYNAMICS.

1. The equation of motion - $\quad$ - $\quad$ - $\quad$ - $\quad-344$

a. The ordinary hydrodynamics $\quad-\quad-\quad-\quad-i b$.

$\beta$. How changed when "internal friction" is taken into $\begin{array}{llllllll}\text { account } & - & - & - & - & & - & 345\end{array}$

2. The ordinary law of resistance $\quad-\quad \quad-\quad 346$

a. How deduced from the equations of motion $\quad-347$

$\beta$. The general results of experiments made since Newton's time compared with the law - $\quad-\quad$ -

$\gamma$. The resistance should be deduced from a rigorous solution of the equations of motion adapted to the case under consideration, - case of the pendulum - 357

3. The resistance to a pendulum - $\quad-\quad-\quad-\quad-i b$.

$a$. Bessel's mode of expressing the resistance - $-i b$.

$\beta$. The careful experiments of Sabine, Baily, Coulomb, \&c. - - - $\quad$ - $\quad$ - $\quad$ -

$\gamma$. Poisson deduces the nature of the motion and the resistance from the ordinary Hydrodynamic equations -

$\delta$. On comparing the theory with experiment they are found not to agree - $\quad-\quad$ - $\quad$ -

$\varepsilon$. Professor Stokes takes into account the effects of $\begin{array}{llllll}\text { internal friction } & - & - & - & & -\end{array} 365$

$\zeta$. The results agree with experiment $\quad-\quad-366$ 
4. The resistance to floating bodies _ _ _ Page

a. The phenomenon of emersion - $\quad$ - $\quad$ - $i b$.

3. Waves are excited in the Fluid - - _ - $\quad$ ib.

$\gamma$. Strange variations of the resistance as the body

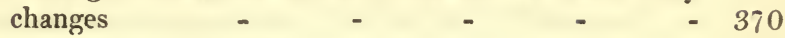

\section{CHAPTER V.}

THE MOTION OF A PENDULUM.

1. Some general considerations - $\quad-\quad-\quad-\quad-240$

2. Motion in vacuo. Newton, xxiv. Note VI. - $\quad-372$

3. The properties of a pendulum. Note VI. - - $\quad 375$

4. Motion in a resisting medium; modern method of considering the perturbations of a pendulum; the Newtonian method - $\quad-\quad$ - $\quad$ - $\quad$ - 242

5. Newton's experiments to discover the law of resistance $\quad-\quad 252$

\section{CHAPTER VI.}

MOTION OF FLUIDS RUNNING OUT OF SMALL ORIFICES.

1. Newton's solution of the question, without limiting the orifice - $\quad$ - $\quad$ - $\quad$ -

2. Newton's corollary - to deduce the resistance made by a fluid to a body moving in it $\quad-\quad$ - $\quad-260$

3. The fallacy of Newton's reasoning - How others attempted to pursue the investigation. Note VII. - -

4. The velocity as given by the Equations of Motion. Note VII.

5. The efflux of an elastic fluid through a small orificestrange conclusions to be deduced from the formula St. Venant and Wantzels' experiments. Note VII. -

\section{CHAPTER VII.}

THE MOTION OF WAVES.

1. General consideration on the nature of Waves - $\quad-266$

2. Waves in air — sound - $\quad$ - $\quad$ - $\quad$ - $\quad$ - 269

a. The nature of sound deduced from the phenomena.

Scholium, Prop. L. $\quad$ - $\quad$ - $\quad$ - $\quad$ - 269

$\beta$. Examination of the case considered by Newton - 271

$\gamma$. Velocity of sound - Newton's error - - $\quad 274$

$\delta$. The manner in which sound spreads after entering through an orifice. Newton, xLII. $\quad-\quad \quad-277$

$\varepsilon$. The notes sounded by different pipes. Scholium, Prop. ‥ 
3. Waves in water

a. The motion is of the vibratory kind

$\gamma$. Velocity of waves. Newton, xLv. \& xLvi. - $\quad 283$

$\varepsilon$. The nature of the motion of waves as given by a strict hydrodynamic theory. Note VIII. - $\quad$ - $\quad 389$

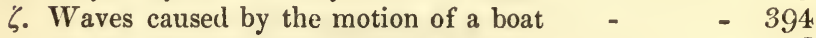

$\mu$. Cause of breakers over sunken rocks - $\quad-396$

$\theta$. How the wind raises the waves - $\quad-\quad \quad-397$

\section{CHAPTER VIII.}

\section{THE THEORY OF THE TIDES.}

I. Newton's investigation on the tides - $\quad-\quad \quad-285$

a. The tides considered as a question in the motion of fluids, deduced from the Lunar Theory - $\quad-286$

$\beta$. General explanations of eight phenomena of the tides 288

$\gamma$. The calculation of the beight of the lunar and solar

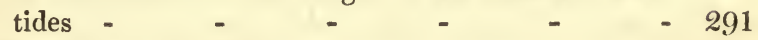

$\delta$. The tides in the moon - $\quad$ - $\quad-\quad$ - 292

II. The theories that have been proposed since Newton's time.

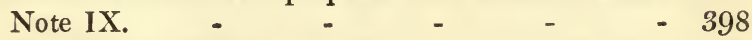

1. The Equilibrium Theory - $\quad$ - $\quad$ - $\quad$ - $i b$.

a. Its fundamental hypothesis $\quad-\quad$ - $\quad$ - 399

$\beta$. The results of calculation made according to this theory, - three kinds of tides - $\quad-\quad$ - $\quad$ - 401

$\gamma$. Airy's opinion of this theory $\quad-\quad \quad-\quad \quad-403$

The Hydrodynamic theory - $\quad$ - $\quad-\quad$ - $i b$.

a. Its fundamental assumptions - - - $i b$.

$\beta$. The results of calculation, - three kinds of tides - 404

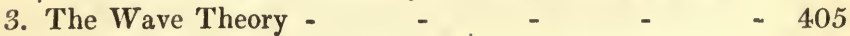

a. Consideration of ocean tides - - - $\quad$ - 406

$\beta$. River tides, results of calculation and explanation of the chief phenomena - - - - $\quad-408$

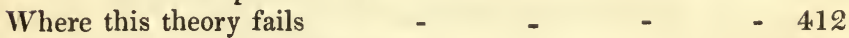

1II. Some results of observation - $\quad-\quad$ - $\quad$ - 413

\section{CHAPTER IX.}

THE CIRCULAR MOTION OF FLUIDS.

1. The hypothesis of the Cartesian theory - - 296

2. Newton's hypothesis as to the law of internal friction in fluids, - the motion of a cylindrical vortex, - Bernoulli's objections to this result. Prop. LI. - $\quad-297$ 
Some difficulties of the Cartesian theory which are considered

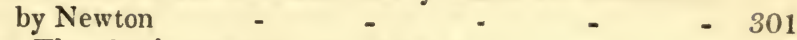

a. The Sun's rotation. Lir., Cor. 4. $\quad$ - $\quad 303$

$\beta$. The third law of Kepler. Scholium Lirr. - $i b$.

$\gamma$. The two first laws of Kepler, Scholium LiII. - $\quad i b$.

$\delta$. The density of the planets, LIII. - - - $\quad i b$.

$\varepsilon$. The disturbance of the sun's motion by the planetary vortices, LII., Cor. 5. \&c. $\quad$ - $\quad-\quad$ - 304

\section{CHAPTER $\mathbf{x}$. \\ THEORY OF COMETS.}

1. The comets are planets moving in very eccentric conic

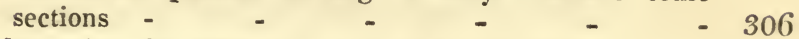

2. To determine the particular orbit of a given comet $\quad-307$

\section{APPENDIX.}

Note I. Galileo's persecution, 417 .

Note II. Another demonstration to p. 58, 420.

Note III. Force varying inversely as the distance, 421.

Note IV. Central forces to more than one point, 424 .

Note V. Leibnitz's Dynamical tracts, 437. 



\section{INTRODUCTI $0 \mathrm{~N}$.}

THE object of this Analytical View is two-fold; first, to assist those who are desirous of understanding the truths unfolded in the Principia, and of knowing upon what foundation rests the claim of that work to be regarded as the greatest monument of human genius - secondly, to explain the connexion of its various parts with each other, and with the preceding and the subsequent progress of the science.

I. It cannot be denied that fully to comprehend the propositions, to follow the demonstrations throughout, requires the reader to be well acquainted with the Mathematics; and even one so prepared, will find the task far from easy in consequence of the synthetical method almost everywhere adopted, the geometrical form of the investigation in many cases where the algebraical would be more convenient, and the extreme conciseness very generally studied, often to the omission of 
many steps in the process.* But it is equally undeniable that with very moderate mathematical acquirements, a distinct and accurate knowledge may be obtained of the fundamental truths unfolded, of the reasoning by which some of them, and these the most important, are sustained, and of the nature of the proof on which the others rest. There is not much difficulty, indeed, in learning those truths, in comprehending the propositions without going further. But this is in every way a most imperfect knowledge, and neither can give satisfaction though retained, nor is likely to be retained without a knowledge also of the demonstrations. A great advantage, however, is gained, if the learner can not only follow the demonstration of the more important propositions, so as to be satisfied of their truth, but can comprehend the nature of the proof in the other instances. He has both made solid progress in mastering the principles of the science, and has become able to judge for himself the merits of the great work which first taught it to the world.

Thus two classes of readers may benefit by this Analytical View; those who only desire to become

* No one needs scruple to confess how difficult he has found the reading of the Principia, when so consummate a geometrician as Clairant has made a like observation, (Mem. Acad. 1745, p. 329.), though somewhat qualified. 
acquainted with the discoveries of Newton, and the history of the science, but without examining the reasoning, and those who would follow the reasoning to a certain extent, and so far as a knowledge of the most elementary parts of geometrical and analytical science may enable them to go. It has been found upon trial that readers of both descriptions have been able to peruse the work with advantage, even readers of the second description. These have easily followed, not only the commentary upon the gradual progress of discovery, and the state of the science before Newton *, but passing over the exposition of the differential calculus $\dagger$ have pursued the demonstration of the fundamental law of gravitation $\ddagger$, and even apprehended the proof of its universal action according to the inverse proportion of the squares of the distances. $\$$ Passing over the detailed discussion of central forces $\|$, the illustration of its application to planets and comets is involved in little difficulty $\mathbf{~ \% ; ~ a n d ~ t h e ~}$ manner of finding the place of these bodies at given periods of their revolution may be understood without entering into the details of the investigation.** The ascertainment of the force

* First 20 pages.

$\ddagger$ P. 31. to 41 .

\|I P. 50. to 64.

**P. 69. to 76 .
† P. 20. to p. 31 .

$\S$ P. 4I. to 50 .

. P. 64. to 69 . 
answering to a given orbit is much easier than the converse of finding the orbit from knowing the force; accordingly the subject of the inverse problem may be passed over.* So may the great head of disturbing forces $\uparrow$, but the interesting history of the problem of three bodies may without difficulty be comprehended. $\$$ The investigation, however, of Lagrange and Laplace of the principle upon which the stability of the system depends, must be taken upon the results without entering into the steps of the process. $\$$ But the wonderful anticipation by Newton of subsequent discoveries may be generally understood and appreciated.\| The subject of the attraction of masses, spherical and others, may be regarded as not coming within this elementary view of the work. 9 The application of dynamical principles to the rays of light**, and the general statement respecting the Newtonian discoveries, and the constitution of the universe, may easily be followed. $\dagger$ Nor can there be great difficulty in understanding the explanation given under the Third Book, of the effects of attractive forces upon the figures of the heavenly bodies, the motions of comets, so far

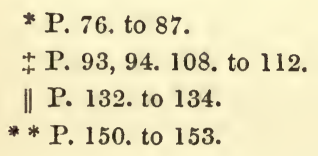

† P. 87. to 108 .

§ P. 112. to 124 .

TP. 134. to 150 .

$\dagger \dagger$ P. 153. to 160 . 
as that subject has not already been dealt with, and the doctrine of the tides, and in tracing the course of reasoning by which these important subjects are investigated.*

The theory of motion in resisting media, and generally whatever relates to fluids, whether their pressure or their movements, forms the next subject of inquiry; but in the elementary view with which we are now occupied, it may be properly passed over, with only this remark, that the matters contained in the Second Book are conveniently described at the end of the account given of the First, although it has been found expedient to follow a different plan of arrangement in the Analytical View of the Second from that originally laid down. The great progress which has been made in hydrodynamics since the time of Newton, has rendered it necessary to enter more minutely into the investigations connected with the Second Book, than into those of the First and the corresponding portions of the Third. Hence this portion of the present work cannot fail to be found less elementary than the former. An acquaintance with the subject, too, is less indispensable towards obtaining a knowledge of the Newtonian philosophy as exposing the system

* P. 285. et seq. 
of the universe, its structure and motions, although it must be sedulously studied by all who would become acquainted with physical science, and all who desire to understand the whole of the Principia.

It has been deemed expedient in giving the propositions of the First Book, to anticipate in some degree their application to the motions of the heavenly bodies, which form the subject of the Third. This course was naturally suggested by the circumstance that the greater part of these propositions bear a distinct reference to the heavenly motions. But it is truly gratifying to find, as we now do, from Sir D. Brewster's valuable Life of Sir Isaac Newton (one of the most precious gifts ever made both to scientific history and physical science), that the illustrious author himself considered this the best method of teaching the Principia to those not thoroughly conversant with mathematics. Applied to by the celebrated Dr. Bentley, who was desirous of so far understanding the book that he might be able to lecture upon its uses in behalf of Natural Religion, he laid down a plan of reading closely resembling that sketched in the beginning of this Introduction; recommending that after the earlier propositions of the First Book, the Third should be taken so far as to perceive its scope, and then such 
parts of the First as " he should have a desire to know, or the whole, in order, if he should think fit." Newton also requires in his correspondent a much more moderate provision of geometrical and algebraical knowlenge than another mathematician laid down as requisite, to whom application had been made for advice, and who gave Dr. Bentley so formidable a list of books as necessary to be read, that he at once appealed to Sir Isaac Newton himself, who prescribed three or four instead of above thirty. (Vol. i. p. 464.)

If it has been made manifest that a very limited acquaintance with mathematics may suffice for attaining a competent notion of the general scope of Newton's discoveries and of the great work which revealed them to the world, it is no less certain that the knowledge thus acquired must be superficial, except as regards the fundamental doctrine of gravitation, the foundation of the system ; and that in order well to understand the dynamical researches which have exercised so mighty an influence upon the whole of Natural Science, a much more full and minute study of the Principia is required. It is to be hoped, therefore, that readers of the two classes referred to, those of the second especially, may be encouraged to pass into the third, for whose use this Analytical View is designed; may make 
themselves, in some measure, masters of the Calculus, the help of which is required in most of the investigations ; and may follow these so as to understand the whole of the propositions, by satisfying themselves of their demonstrated truth. That this also can be done with only a previous knowledge of elementary geometry and algebra, has been proved upon trial, that knowledge sufficing to attain an acquaintance with the rules explained in those parts of this treatise above recommended to be passed over by the more general reader. It is by no means intended to affirm that a complete knowledge of the Principia can be attained without much further study. An intimate familiarity with the Calculus, or with the analogous method of Limits, is required by those who desire to follow the whole of the demonstrations, and to perceive the connexion between the different steps as clearly as they can trace those of any elementary process in geometry or algebra. Other helps than this work are required, and are not wanting, to facilitate the entire mastery of the subject by such as would thoroughly understand the Principia in all its parts. The present treatise is not designed for their use, further than as it may aid them in the earliest part of their studies. It is intended for those who may not be able, or may not be disposed, to go beyond acquiring such a knowledge of the 
subject and of the Book, as can be attained by a moderate degree of labour, and an acquaintance with only elementary mathematics.

II. The accomplishment of the other object of this Treatise, examining the connexion of the different parts of the Principia with each other, and with former and subsequent discoveries, showing its transcendent merits, and removing the objections, or rather the criticisms, that have sometimes been offered upon a few comparatively unimportant portions of the great work, will, beside performing that service, also afford additional help to the study of it, and tend to promote the taste for understanding it, so as to judge of its unparalleled excellence. It is satisfactory to find that many of the propositions are capable of demonstration by a process different from that employed by Newton, especially when this process is more easily followed. In many cases the analytical substituted for the synthetical method, is interesting as a matter of curiosity, independently of its more didactic character. This may also be predicated of the occasional preference of algebraical to geometrical reasoning. The greatest interest, however, belongs to observing the mutual bearings of the propositions, perceiving sometimes how one arises out of another, sometimes how the two are so connected that together they exhaust the subject, sometimes how the 
establishment of one mere general truth furnishes the proof of others less general which had been previously reached by a different route; often to mark the diversities as well as resemblances of propositions, and the particular circumstances upon which these severally depend; not rarely to note in what way others had imperfectly obtained the knowledge of these truths, or altogether had failed to observe them; frequently to find them deriving new support from things afterwards brought to light, and to see them explain phenomena subsequently for the first time observed; above all, to see, and as we see to marvel how, beside those doctrines, the teaching of which forms the main object of the work, which are expounded with an exhaustive fulness, and are at their first discovery established in absolute perfection, so that scarce any addition has, in the vast majority of instances, been found either possible or required, there are also the foundations laid of new discovery in other directions, the rudiments provided of other systems, and the very course plainly pointed out by which these unthought of truths should in remote ages be explored.

On some few points differences of opinion having arisen as to other men's claims to the discovery, all controversial matters are purposely avoided. But although it must be confessed that philoso. 
phers, as well in foreign countries as among ourselves, have shown no reluctance to allow Newton's title to the first place, there have occasionally been criticisms hazarded rather than objections made, touching several parts of his great work; and these in most cases have originated rather in inadvertence than in any unworthy prejudice. It became necessary to correct such errors, in justice to the illustrious author, who could not have been aware of the statements, except perhaps in one instance. It is possible he might have known the groundless remarks of $\mathrm{J}$. Bernouilli in that particular; the equally erroneous statements of Bailly and Laplace of course he could not have been aware of, and we may confidently add, never could have foreseen; indeed they could only be ascribed to oversight in those eminent authors. The error detected by F. Boscovich respecting the comet's path belongs to another class, and arose entirely from a neglect of that careful examination of the limits of a problem peculiar to the ancient analysis, that exhaustive process by which the prolixity sometimes complained of finds ample compensation in its precluding the possibility of mistake.

Thus it is hoped that, partly by the account of the work and partly by discussions connected with the subjects of which it treats, the study 
of it may be both promoted and facilitated; and this kind of service towards the progress of science is not to be altogether contemned. They who are incapable themselves of advancing it by the discovery of new truths, may usefully employ themselves in helping others to a knowledge of what the great masters have done; and they may best do this if they shall not disdain the office of elementary explanation and discussion. The wisest of the ancients was said to have brought Philosophy down from heaven to earth; he certainly valued himself chiefly on his unceasing efforts to stir up in men's minds the desire of knowledge. What he found necessary with regard to the nature of the subject, we in our day may perceive to be equally necessary because of the clouds in which writers of vast and original genius almost unavoidably involve the records of their inquiries after unknown truths.

But whatever brings men acquainted with those profound researches, raises their minds to contemplations far more sublime than any which are connected merely with worldly science. To survey the most wonderful works of creation, to comprehend the laws by which the system of the universe is governed, the principles which everywhere pervade it, and bear irrefragable testimony to the unity as well as the power of the divine Author and Dis- 
poser of all, is the most impressive lesson that unassisted reason can teach our species. It is an observation of Paley, marked with his wonted sagacity, that though Physical Astronomy, until well understood, presents less striking proofs of design to the mind than the other branches of science, yet when fully apprehended, it very far exceeds all the other evidences of Natural Religion. We must recollect, too, that Newton himself regarded this as the most precious part of his philosophy; declared that in framing it he had been moved by a desire to inculcate religious belief, expressed his gratification in finding that his efforts had not been vain, and closed the exposition of its principles with a commentary upon the nature and attributes of God. His followers may be permitted to indulge the hope that he would have prized their humble attempts at diffusing a knowledge of his immortal labours, rather as falling in with his pious wishes for the happiness of others, than as contributing to the illustration of a fame which is imperishable, nor admits of any increase. 


\section{ERRATA.}

Page 33. In the figure between $\mathrm{S}$ and $c$, put $\mathrm{C}$ at end of line $\mathrm{V}$.

Page 42. In the figure at end of PS, put V.

Page 47. line 9, for Y P read Y S ; and after "circle," transpose, "and joining VF" to before "we."

Page 48. line 10, add " and the Fathers fall into the same error." I. 109. 


\section{NEWTON'S PRINCIPIA.}

\section{ANALYTICAL VIEW.}

THIS work is justly considered by all men as the greatest of the monuments of human genius. It contains the exposition of the laws of motion in all its varieties, whether in free space or in resisting media, and of the action exerted by the masses or the particles of matter upon each other, those laws demonstrated by synthetic reasoning; and it unfolds the most magnificent discovery that was ever made by man - the Principle of Universal Gravitation, by which the system of the universe is governed under the superintendence of its Divine Maker. Two of the three Books into which the treatise is divided are chiefly composed of mathematical investigations, conducted by the most refined and profound, but at the same time the most elegant application of geometry, and of a calculus which is only a particular form of the fluxionary method invented by the illustrious author in his early years. The Third Book contains an explanation of the motions of the heavenly bodies, deduced chiefly from the first portion of the former part, and grounded upon the 
phenomena observed by astronomers. This concluding portion, however, of the great work, is also interspersed with geometrical reasoning of the same admirable description as characterized the former, and applied to the solution of problems respecting the heavenly motions.

Before Sir Isaac Newton appeared to enlighten mankind, and to found a new era in the history of physical science, the eminent men who had preceded him had made, during the century immediately preceding his birth, very important steps in furthering the advancement of our knowledge; and they had approached exceedingly near that point which forms the most important of all his discoveries, according to a kind of law which seems to regulate the progress of human improvement-a law of continuity, which apparently prevents any sudden, and, as it were, violent change, from being made in the intellectual condition of the species, and prescribes the unfolding of all great truths by slow degrees, each mighty discovery being preceded by others only less considerable than itself, and conducting towards it. The great discoveries in pure mathematics afford striking examples of this truth. That of Logarithms by Napier is, perhaps, the instance in which the most considerable deviation has been made from the rule; but even here there had been some curious methods of mechanical calculation invented before, and the discoverer of logarithms himself had reached the point very nearly by other most ingenious contrivances, before he actually made his great step.

But the Fluxionary or Differential Calculus gives a remarkable exemplification of the general principle; and its subsequent most important extension, the Calculus of Variations, furnishes another not less striking. Ever since Descartes's happy application of Algebra to Geometry had 
opened the way to the grand discovery of Newton and Leibnitz, the foundation of modern science, mathematicians had been intent upon the resolution of problems connected with the rectification and quadrature of curves, and the determination of points that possess properties of maxima and minima, as well as the finding of normals, tangents, and osculating circles. These inquiries had led them to consider the laws by which the relations between the ordinates and abscissæ referred to any given axis are governed at different points of that axis; for in truth that implies the nature of the curvature itself, and includes the manner in which the length of the curve line increases or diminishes, as well as the space which it incloses. They were thus led to examine the generation of those curve lines and curvilinear spaces, whether that is conceived to be effected by the movement in the one case of points, and in the other of straight lines, or is supposed to be produced by the constant juxtaposition of indefinitely small straight lines inclined to each other according to a given law, in the one case, and indefinitely small rectangles in the other. The latter is perhaps the more natural supposition of the two, and not the less easy. For if any one is set to measure the area of a field bounded by a curvilinear outline, as he can at once measure a space inclosed within straight lines, his course will be to divide the given space into rectangles, and then to divide each of the smaller curvilinear spaces into other rectangles, and so on till he has exhausted the whole by a series of rectangles, always decreasing in size as they increase in number, and the last of which seem to coincide nearly or sensibly with the area of the outer or curved line of boundary. Thus he would proceed by trial and actual measurement of the space; and thus do land-measurers (the lineal descendants of the first 
geometers, as well as their namesakes) still proceed. But speculative mathematicians being aware of the general properties of the lines they have to examine, and these being regularly formed, which the boundary of the field is not, they could calculate the relations to each other of the sides of the rectangles into which they divided the figure, and could thus form series of rectilinear figures diminishing in size, and which series might be carried to any length so as ultimately to exhaust the curvilinear area. Thus $\mathrm{ABC}$ being a semicircle, it was easy to find the area

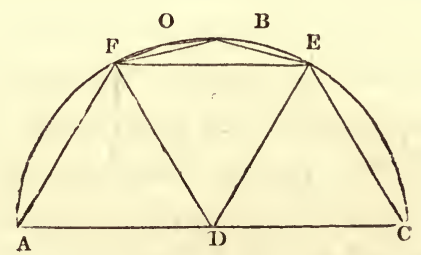

of the semihexagon or three equilateral triangles $\mathrm{ADF}$, $\mathrm{FDE}$, and $\mathrm{DEC}$, and then of the triangles $\mathrm{FBE} \times 3$, and again of the triangles $\mathrm{FOB} \times 6$, and so on; so that the radius $\mathrm{AD}$ being called $r$, there was obtained a series of this form, $\frac{3}{2} r^{2} \sqrt{3}+\frac{3}{2} r^{2}(2-\sqrt{3})+\frac{3 r^{2}}{2} \sqrt{2}$ $(2 \sqrt{3}-2-\sqrt{2})+*$ \&c.: And thus we have also the approximation to the length of the circle.

But the extreme cumbrousness of this calculus, which is still more unmanageable in other curves where the radii are not, as in the case of the circle, equal, made it necessary to find some other method; and geometricians accordingly examined the laws by which the areas increase in each curve, so that by adding all those innumerable increments together their sum might give the exact space

* The three first terms give 3.10582 ; the seven first come very near the ordinary approximation, $3.14159,-$ for they give 3.14144 . 
required. The same process was attempted with the lengths of the curves, considering them as polygons whose sides diminished while their numbers increased indefinitely. In this way Cavalleri, Fermat, and Wallis, and still more Harriot and Roberval, appear to have come exceedingly near the discovery of the general rule for performing these operations before Newton and Leibnitz, unknown to each other, made the great step. Roberval especially had solved many problems of quadrature and of drawing tangents, by methods extremely similar to the Newtonian. Nor were the ancient methods of Exhaustion and Indivisibles so far distant as to let us doubt that, had the old geometers been possessed of the great instrument of algebra, and bethought them of its truly felicitous application according to the idea of Descartes, long before our times they would have anticipated the discoveries which form the great glory of modern science.*

The discovery of the Calculus of Variations affords a similar example of gradual progress. When the differential calculus had enabled us to ascertain the maxima and minima of quantities, for example the value of one co-ordinate to a curve, at which the other becomes a maximum or a minimum, or, which is the same thing, the point of greatest and least distance between the curve and a given right line, or, which is the same thing, when the general relation of the co-ordinates being given we were enabled by means of the calculus to examine what that particular value was at which a maximum property belonged to one of them - then geometricians next inquired into the maxima and minima of different curves, that is to say, into the general relation between the co-ordinates which gave to every

* Among other marvels in Galileo's history he seems to have made a near approach to the calculus. - See M. Libri's most able and learned work, Hist. de Math. en Italie, tom iv. 
portion of the curve a maximum or a minimum value in some respect. Thus, instead of inquiring at what value of $x$ (the abscissa) in a known equation between $x$ and the ordinate $y, y$ became a minimum, or the curve approached the nearest to its axis, the question was what relation $x$ must have to $y$ (or what must be the equation as yet unknown) in order to make the whole curve, for example, of the shortest length between two given points, or inclose with two given lines the largest space, or (having some property given) inclose within itself the largest space, or be traversed in the shortest possible time by a body impelled by a given force between two given points. Here the ordinary resources of the differential calculus failed us, because that calculus only enabled us, by substituting in the differential equation the value of one co-ordinate in terms of the other, to make the whole equal to nothing, as it must be at the maximum or minimum point where there is no further increase or decrease. But here no means were afforded of making this substitution, and the problem seemed, as far as this method went, indeterminate. Various very ingenious resources were employed by Sir Isaac Newton, who in the Principia seems to have first solved a problem of the Isoperimetrical class - that is, finding the solid of least resistance; and soon after by the Bernouillis and other continental mathematicians, who worked by skilful constructions and suppositions consistent with the data. The calculus called that of Variations has since been invented for the general solution of these and other similar problems. It consists in treating the relations of quantities, or of their functions, as themselves varying, but varying according to prescribed rules, just as the differential calculus regards the quantities themselves, or their functions, as varying according to prescribed rules. It bears to the differential 
calculus somewhat of the relation which that bears to the calculus of fixed and finite or unvarying quantities.

It is wonderful how very near Bernouilli, when he solved the problem of finding the line of swiftest descent, came to finding out this calculus; if, indeed, he may not be said to have actually employed it when he supposed, not as in the case of the differential calculus, two ordinates of a known curve infinitely near one another, but three ordinates infinitely near, including two branches of an unknown curve, each infinitely small; for he certainly made the relation of these ordinates to the abscissa vary. Euler used the calculus more systematically in the solution of various problems; but he was much impeded for want of an algorithm. This important defect was supplied by Lagrange, who reduced the method to a system and laid down its general principles; but had Euler gone on a little step further, or had Bernouilli been bent on finding out a general method instead of solving particular problems, or had Emerson, who has one or two similar investigations in his book on Fluxions, reduced the method by which he worked them to a system by giving one general rule (which, writing a book on the subject, he was very likely to have done), the fame of that discovery would have been theirs, which now redounds so greatly and so justly to the glory of Lagrange.

The discovery of Gravitation as the governing principle of the heavenly motions, is no exception to the rule which we have stated of continuity or gradual progress. When Copernicus had first clearly stated the truth to which near approaches had been made by his predecessors, from Pythagoras downwards, that the planets move round the sun, and that the earth also moves on its axis while the moon revolves round the earth, he yet 
accompanied his statement with so little proof beyond the agreement with the phenomena, which the Ptolemaic hypothesis could equally boast of*, that for more than half a century afterwards it had no general acceptance, Bacon himself rejecting it; when Galileo, by his telescopic discoveries, especially of the phases of Venus and the satellites of Jupiter, and by his yet more important discoveries in the laws of motion, may be said first to have proved the truth of the Copernican system. Afterwards the satellites of Saturn, added to Kepler's observation of Mercury's transit over the sun, afforded most important confirmation. The great discoveries of this eminent man followed close after those of Galileo: First, the motions of the planets were found to be in ellipses with the sun in one focus; secondly, lines drawn to the sun from them were found to describe areas proportional to the times of their revolution; and, thirdly, the relation was established between the squares of those times and the cubes of the distances of the bodies from the focus.

How near this brought scientific men to the cause or law of the whole is manifest, especially when we regard the connexion thus established between the revolving bodies and the great luminary in the centre. Although Kepler himself erroneously mingled with the influence which this law of motion led him to ascribe to the sun, a transverse force which he deemed necessary to maintain the projectile motion of the planets round the centre; yet others formed more correct ideas of the matter. - It seems to have been Huygens, who, fourteen years before the "Principia" was published, first showed the true nature of centrifugal forces. - Several years earlier, however, Borelli, in treating of the motion of Jupiter's

* It is certain that its greater simplicity was, before Galileo's time, the only argument in favour of the Copernican theory against the Ptolemæan. 
satellites, considers the planets as having a tendency to resile from the sun and the satellites from the planets, but as being "drawn towards and held by those central bodies, and so compelled to follow them in continued revolutions." He also most accurately compares the receding (or centrifugal) force with the tendency of a stone whirled in a sling to fly off at every instant of its motion.Hooke, a man of unquestionable genius, and whose partial anticipations of many great discoveries are truly remarkable, about the same time with Borelli, asserted that the attraction of the sun draws away the planets from moving in straight lines, and that the force of the attraction varies with the distance. He had, as early as 1666 , read to the Royal Society a paper explaining the curvilinear motion of the planets by attraction.- Halley, as well as others, had even hit upon the inverse duplicate ratio, by supposing that the influence from the sun was diffused in a circle, or rather a sphere, and that therefore the areas proportioned to that influence were as the squares of the radii, and that consequently the intensities, being inversely as those areas, were inversely as the squares of the radii or distances. - Finally, Hooke had foretold, that whoever set himself to investigate the subject experimentally would discover the true cause of all the heavenly motions.

Such were the near approaches which had been made to the law of Gravitation before its final and complete discovery. But although in this gradual progress it resembles almost all the other great improvements in science, in one material respect it differs from them all. The theory was perfect which Newton delivered, and the whole subject was at once thoroughly investigated. It was not merely that the general principle hitherto anxiously sought for, and of which others had caught many glimpses, was now unfolded and established upon appropriate founda- 
tions; but almost every consequence and application of it was either traced, or plainly sketched out; it was pursued into all the details; a systematic account of its operation was given, symmetrical, and in its main branches complete; so that, however nearly former inquirers had approached the general law, the distance was prodigious between their conjectures, how learned and happy soever, and the magnificent work which the genius of Newton had accomplished.*

It must be observed, too, that, beside this grand achievement, the Principia performed three other most important services to physical and mathematical science. First. It laid a deep and solid foundation for subsequent discoveries in the science of physical astronomy, both by the general principles of dynamics which it unfolded, and by the application which it made of these to the heavenly bodies and their motions. Secondly. It gave a complete system of dynamics applicable to all subjects connected with motion and force and statics - a system throughout abounding in the most important original mathematical truths, expounded and proved with singular beauty, though with extreme conciseness. Thirdly. It propounded and showed the application of a new calculus, or method of mathematical investigation, that method by the help of which those truths had been discovered; and by which others, before resting upon an empirical foundation, were demonstrated. Thus it is no exaggeration to say that, even if the great dis-

* The subsequent discoveries of mathematicians by means of the improvements in the calculus, have added new illustrations, and traced further consequences of the theory. But there is only one of their improvements which can justly be said to have advanced the evidence of the fundamental principle further than Sir I. Newton had carried it, by supplying any defect which he had left; we allude to the reconcilement by Clairaut of the moon's apogeal motion according to the theory with the observations. This is fully explained in the sequel. It forms one of the most interesting passages in the whole history of science. 
covery of the law which governs the universe were taken away from the Principia, it would still retain its rank at the head of all the works of mathematicians, as the most wonderful series of discoveries in geometrical science, and its application to the principles of dynamics.

That the reception of this work was not such as might have been expected has frequently been alleged; and although an ingenious and well-meant attempt has lately been made by an eminent author* to relieve this country from its share of the imputation, chiefly by showing the estimation the author was held in immediately after its publication; it is, on the one hand, certain that Newton's previous fame was great by former discoveries, and that after its appearance the Principia was more admired than studied. There is no getting over the inference on this head which arises from the dates of the two first editions. There elapsed an interval of no less than twenty-seven years between them; and although Cotes speaks of the copies having become scarce and in very great demand when the second edition appeared in 1713, yet had this urgent demand been of many years' continuance, the reprinting could never have been so long delayed; nor was the next edition required for thirteen years after the second. So that in forty years the greatest work ever composed by man reached only a third edition; and that third has, during the succeeding hundred years, been the one generally in use; although translations and excerpts have been published from time to time, and two editions were printed on the Continent, one at Amsterdam and one at Cologne. The doctrines of the work were, however, much more readily embraced and more generally diffused in this country, which had the benefit of Maclaurin's ad- 
mirable view of the more general principles of the system, publiabed about the middle of the last century. On the Continent they made their way far more slowly; nor was it until Voltaire employed his great powers of clear apprehension and lucid statement to give them currency, that the Carterian prejudices of our neighbours gave way, and the true doctrine found a general and a willing acceptance.

It must be adruitted that the manner in which the truths of the Principia were unfolded, not only added somewhat to the slowneis of the world at large in embracing them, but has also contributed to the reluctance with which men have generally undertaken the task of reading that great work, and eaticfying themselves of the proofs upon which its doctrines rest. Conciseness is everywhere rigorously studiod. Not only does the author avoid all neodless prolixity and repetition in unfolding his discoveries, but he leaves out so many of the steps of his demonstration, and assumes his reader to be so expert a geometrician, that the labour of following him is often sufficient to deter ordinary students from making the effort. If mathematical reading is never the same passive kind of operation with other studies, the perusal of the Principia is emphatically an active exercise of the mind. For what, to the intuitive glance of him who could discover the theorem or solve the problem, appeared too plain to require any proof, may well stop common minds in their progreas towards the point whither he is guiding them; the dis tancess which he can stride at once over this difficult path must, by weaker persons, be divided into many portions, and travelled by successive steps. Add to which, that, as the method of proof is throughout synthetical, and as it is geometrical, the helps of modern analysis are thus withlield. Upon the whole, therefore, a most valuable 
service was rendered to students by the able and learned commentary of the Fathers, Le Seur and Jacquier, who, in 1739 and 1742, published the Principia, with very copious illustrations, although it is to be regretted that they rosort far less frequently to analysis than was desirable. It is remarkable enough, and affords an additional proof of the slow progress which truth had then made in some parts of Europe, that these excellent authors deemed it nocessary to accompany their publication of the Third Book, which treats of the hearenly motions, with a declaration in these words: "Newtonus in hoc tertio libro Telluris mota hypothesim asserit. Autoris propositiones aliter explicari non poterant, nisi câdem quoque facta hypothesi. Hine alienam coacti sumus gerere personam; caterum latis a summis Pontificibus contra Telluris motum Decretis nos obsequi profitemur." This edition is dated, as might be supposed, at Rome.*

The Principia begins with a definition of terns, and a compendious statement of the science of dynamics as it existed previous to Newton's discoreries. The definitions. eight in number, comprise that of quantity of matter, which is in the proportion of its bulk and density, the density being the proportion of its mass to its bulk - the quantity of mofion, which is in proportion to the relocity and quantity of matter jointly - the eis inertice, which is

* It must, howeror, be observed, that sach bigotry and intolerance mas not confined to Rome. As late as 1769, biaffom was compelled, br the interference of the Sorbonne, to pahlish a recantation of some portion of his fantastical shoory of the caryh, comprehending, as it happencu, the rery fer things in it which had any rosisonalle foundarion. We onght also to mention, for the eredit of the Parkal Goreroment, that a late peontiff (Pivs VII.) procured a repoal of the docree Against the Coporwican srstem. 
the force or power of matter to persist in any given state, whether of rest or of motion in a straight line, and to resist any external force impressed upon it to change that state-centripetal force, which is the power that draws towards a given point or centre bodies at a distance from it - finally, the three kinds of amount of centripetal force; the absolute amount, in proportion to the intensity of the power exerted in drawing towards the centre; the accelerating, in proportion to the velocity generated in a given time; and the moving, in proportion to the motion generated in a given time towards the centre.*

Two things are worthy of remark in these definitions: first, that, as if foreseeing the cavils to which his doctrines would give rise, he guards, in a scholium, against the supposition that he means to give any opinion respecting the nature or cause of centripetal force, much less that he ascribes any virtue of attraction to mere centres or mathematical points; whereas he only means to express certain known and observed facts: secondly, that, in illustrating his definition of centripetal forces, he really anticipates his great discovery; for, after giving the examples of magnetic action, and of a stone whirled in a sling, he proceeds to the motion of projectiles, and shows how, by increasing the centrifugal force, they may be made to move round the earth, as may also, he says, the moon, if she be a heavy body, or in any other way be deflected towards the earth, and retained in her orbit. That force, he adds, must be of a certain amount, neither more nor less; and the business of mathematicians is to find this necessary amount; or, conversely, having the amount

* There are eight definitions in the book, though we have only given them under seven heads, not having made a separate definition of the force impressed, which is here mentioned under the important head of the vis inertia. 
given, to find the curve in which it makes the body move. The connexion between the inquiries which form the main subject of the two first books of the Principia and Physical Astronomy, the subject of the third, is thus explicitly stated; but a plain indication is also here afforded of the great discovery in which the whole investigation is to end.

The doctrines of dynamics, known previously to his discoveries, are then given in the form of corollaries to the three general Laws of Motion. The first law is that of the vis inertice, already explained; and it is to be observed here that a steady and clear conception of the tendency of all moving bodies to proceed in a straight line unless deflected from it, is, perhaps, more than anything else, that which distinguished the New tonian from the immediately preceding doctrines, mixing up as these did more influences than one proceeding from the centre with a view to explain the composite motion of the planets.

The second law is, that all changes in the motion of any body, or all changes from rest to motion, are in proportion to the moving force impressed, and are in the straight line of that force's direction.

The third law is, that reaction is always equal and opposite to action; or that the mutual actions of any two bodies are always equal to one another, and in opposite directions.

From these laws the six corollaries which are added deduce the fundamental principles of dynamics; and there is a scholium to the whole, which states the application of those principles to the descent of heavy bodies, and the parabolic motion of projectiles. Of all the principles, the most important is that of the Composition and Resolution of forces. As by the first law a body always 
perseveres in the straight line it moves in, unless in so far as some other force alters its direction; and as by the second law any new force impressed tends to move it in its own direction, - it follows that, if two forces, not in the same nor in directly opposite directions, act at one time, and by an instantaneous impulse, on any body at rest, it must move in such a direction as that it shall be found both in a line parallel to the direction of the one force, and in a line parallel to the direction of the other; that is to say, in the diagonal of a parallelogram whose two contiguous sides are in the directions of the two forces, and are respectively equal to the space each force would carry it through in its own direction. Moreover, as each force separately would have carried it to the end of the line of its direction in the given time, it must move through the diagonal in the same time which it would have taken to move through either side if either force had acted alone. Thus the direction of every motion occasioned by any two forces acting at an angle to each other, may always be found by completing the parallelogram of which the directions of those forces are the contiguous sides; and so of any motion occasioned by any number of forces whatever acting angularly. And, conversely, every motion of a moving body may be resolved into two, of which the one is in any given direction whatever, and the other is found by completing the parallelogram, whereof that given direction is one of the sides, and the direction the body moves in is the diagonal.

From this resolution of forces it is easily shown, that if any weights or other powers acting in parallel lines are applied to the opposite ends of a lever moving on a centre or fulcrum, the effect of each will be directly as its distance from that centre, in other words, as the length of the contiguous arm of the lever; consequently, that if the 
weights or powers are made inversely as those lengths, the whole will be in equilibrio or balanced. This is the well known and fundamental principle of the lever, the foundation of mechanics; and it applies also to the wheel and axle and the pulley. The fundamental properties of the screw, the wedge, and the inclined plane are deduced in like manner from this important proposition. So may all the properties of the centre of gravity, and the method of finding it; for, in fact, the fulcrum of the lever is the common centre of gravity of two bodies equal to the two weights, and placed at the opposite ends of the lever; and the line joining the bodies is divided in the inverse proportion of those bodies. It also is easily shown that the common centre of gravity of two or more bodies is not moved, nor in any way affected, by their mutual actions on each other, but it either remains at rest, or moves forward in a straight line. So are the relative motions of any system of bodies, whether the space they occupy is at rest, or moves uniformly in a straight line.

The Scholium to the Laws of Motion first considers very briefly the motion of falling bodies which descend with a velocity uniformly accelerated, that velocity which is given to them by the attraction of the earth during the first instant continuing and having at each succeeding instant a new impulse added. The acceleration, therefore, is as the time; and they move through a space proportional to the velocity and the time jointly, consequently proportional to the square of the time, since the velocity is itself proportional to the time.*

* Velocity is as time, i. e., $v$ is as $m t$; space is as relocity $\times$ time, or $s$ as $v \times t$; therefore space is as time $\times$ time, or as square of time, that is, $s$ is as $m t \times t$, or $m t^{2}$.-The proportion of the space fallen through by the force of gravity (or moved through by any body uniformly accelerated) to the square of the times, is also demonstrated thus. Let the 
The Scholium next, with equal brevity, states the projectile motion of heavy bodies. If a body be impelled in one direction by a force producing a uniform motion, and in another direction at any angle with the former by a force not uniform but accelerated, the diagonals which it will move through will at every instant change their direction towards the quarter to which the accelerating force tends. But a series of such diagonals is a polygon of an infinite number of sides, infinitely small: in other words, a curve line. Now in the case of a projectile, this continued or accelerating force is such as to make the body, if no other force acted on it, fall through spaces proportional to the square of the times.

velocity acquired at any moment $\mathrm{P}$ of the time $\mathrm{A} P$ be $\mathrm{P} M$, and because the velocity uniformly increases, or as the time, $\mathrm{PM}: \mathrm{AP}: \mathrm{BC}: \mathrm{A}$,

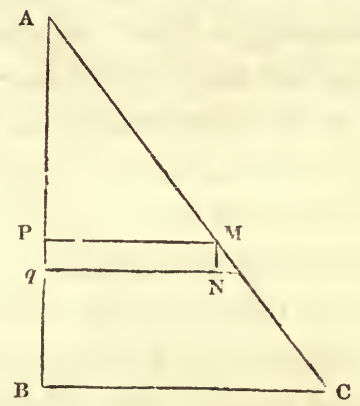

and therefore the line $\mathrm{A} \mathrm{C}$ is a straight line, and the triangles A P M, $\mathrm{A}$ B C, are similar. But if $q \mathrm{~N}$ is infinitely near $\mathrm{P} \mathrm{M}$, or $\mathrm{P} q$ represents the smallest conceivable time, the motion during that time may be conceived to be uniform and not accelerated. Now the space through which any body moves is as the velocity multiplied by the time $(s=v t)$, therefore the space moved through in the time $\mathrm{P} q$ is as $\mathrm{P} q \times q \mathrm{~N}$. So the space moved through in the time $\mathrm{AB}$ will be as the sum of all the small rectangles $\mathrm{P} q \times \mathrm{N} q$, or as the triangle A B C. But the triangle A B C is to any other of the triangles $\mathbf{A P M}$ as $\mathbf{A B}^{2}: \mathbf{A P}^{2}$; therefore the spaces are as the squares of the times. The great general importance of this proposition which Galileo first proved, makes it necessary to have the demonstration clearly fixed in the reader's recollection. 
The other force acting once for all would make it, were there no gravity acting, move in spaces proportioned to the times simply. The latter or projecting force would make it move through A B uniformly, or in spaces proportional to the times; the force of gravity would make it move

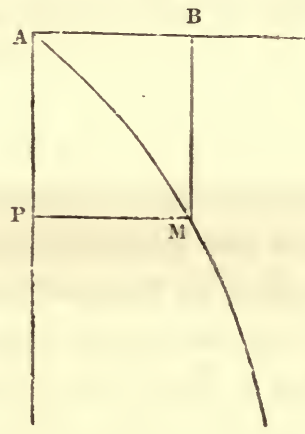

through $\mathrm{AP}$ with a motion proportioned to the square of the times; therefore it will move in a curve passing through $\mathrm{M}$, if $\mathrm{PM}$ is equal, and parallel to $\mathrm{AB}$; and $\mathrm{AP}$ will be as the square of $\mathrm{AB}$ or $\mathrm{PM}$, which is the property of the conic parabola $m \cdot \mathrm{AP}=\mathrm{PM}^{2}, m$ being the parameter to the point $A$.

The Scholium concludes by stating some consequences of the equality of action and reaction, the third Law of Motion, with respect to oscillation and impact, and also with respect to mutual attractions; of which consequences the most important is that the attraction or weight of heavy bodies in respect of the earth, and of the earth in respect of them, is equal.

The great work itself, after these preliminary though essential matters, proceeds to its proper subject. But in order to show how the demonstrations are conducted, a short treatise is prefixed upon the method of Prime and Ultimate Ratios, in eleven Lemmas, with their corollaries. This method consists in considering all quantities as 
generated by the uniform progression or motion of other quantities, and examining the relations which the smallest conceivable spaces thus generated by this motion bear to one another, and to the spaces generated at the moment of their inception, or when they are nascent, which is termed their prime ratio, and at the moment of their vanishing, or when they are evanescent, which is termed their ultimate ratio. Thus a point moving along in a straightforward direction generates a straight line; a line moving parallel to itself, or two. lines moving at right angles to one another, generate a rectangle: one line moving, while a point in it moves along it so that its progress on the moving line always bears a given ratio to the progress the line has made $(m . \mathrm{AP}=\mathrm{PM})$, describes a triangle; the same motion, if the progress of the point bears a variable

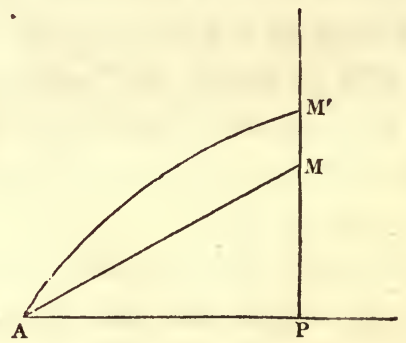

relation to that of the line $\left(\mathrm{x} \cdot \mathrm{AP}=\mathrm{PM}^{\prime} ; \mathrm{x} \cdot \mathrm{x} \mathrm{AP}\right.$ being some function of $A P$ ), describes a curve line and curvilinear area; and so of solids, which are generated by the motion of planes.

It follows from this mode of generation that if the length of any curve line be divided into an infinite number of lines, the sum of these will not differ from the curve line by any assignable quantity, nor will each differ from a straight line; and if its area be divided into an infinite number of smaller areas by lines drawn parallel to the 
line whose progressive motion generated the curvilinear area, the sum of these infinitely narrow areas will differ from the area of the curve by a difference less than any assignable quantity, nor will each differ from a rectangle; in other words, the ratio of the nascent curve line and nascent curvilinear area will be that of equality with the small lines and small rectangles, and the ultimate ratio of the sums of the lines and rectangles to the whole curve line and curvilinear area, respectively, will be that of equality :- Or to put it otherwise, if the axis of the curve be divided into parts $\mathrm{P} P, \& \mathrm{c}$., and the area into spaces PMRP, \&c., by ordinates PM, PR, \&c., and the number of these spaces be increased, and their breadth $\mathrm{PP}$ be diminished indefinitely, which is the operation of the

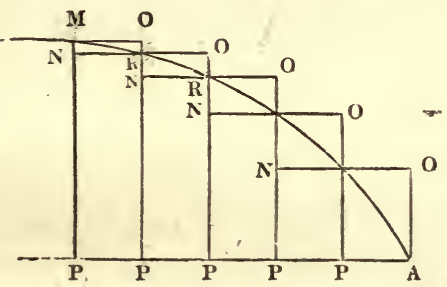

generative motion of PM, the size of each of the small spaces MNRO (by which the curvilinear areas differ from the rectangles) diminishes indefinitely, and the ultimate ratio of all the curve areas PMRP, and all the rectangles PNRP, becomes that of equality, and therefore the sum of evanescent differences NMOR, NROR, \&c., whereby the whole curvilinear area differs from the whole amount of the rectangles $\mathrm{PNRP}$, becomes less than any assignable quantity, or the curvilinear area coincides with the sum of the rectangles. And so of the sum of all the diagonals $M R, R R, \& c$. , which becomes the curve line MRA.

Hence we infer that the amount of these small spaces 
or quantities NMOR, formed by multiplying together two evanescent quantities, is as nothing in comparison with the rectangles $\mathrm{P}$ MO P formed by only one evanescent quantity multiplied into a finite quantity, and may be neglected in any equation that expresses the relations of those rectangles with each other. But if some other quantities be found which are, in comparison with these small ones, themselves infinitely small, the areas formed by multiplying this second set of small quantities may be rejected in any equation expressing the relations of those first small quantities.

Thus we have the origin and constitution of quantities which in the Newtonian scheme are called fuxions of different orders, because conceived to express the manner of the generation of quantities by the motion of others, and in Leibnitz's language are called infinitesimals or differences, because conceived to express the constant addition of one indefinitely small quantity to another. Obtaining the fluxions, or the differences, from the quantity generated by the motion or by the addition, is called the direct method; obtaining the quantity generated from the fluxions, or finding the sum of all the differences, is called the indirect method. The one theory calls the direct method that of finding fuxions, the indirect that of finding fuents; the other theory calls the former differentiation, or finding differentials, the latter integration, or finding integrals. The two systems, therefore, in no one respect whatever differ except in their origin and language; their rules, principles, applications, and results, are the same.

A different symbol has been used in the two systems; Newton expressing a fluxion by a point or dot, and the fluxion of that fluxion, or a second fluxion, by two dots, and so on. Leibnitz prefixes the letter $d$, and its powers 
$d^{2}, d^{3}, \& c$., instead, to express the differentials. In like manner $\int$ for sum is used by the latter to express the integral, and $f$ by the former for the fluent. Although the continental method of notation is now generally used, and is on the whole most convenient, yet it has its inconvenience, as the $d$ is sometimes confounded with co-efficients of the variable quantities; it is in some respects, too; not very consistent with itself; as by making $d x^{2}$ mean the square of the fluxion, or differential of $x$; whereas it, strictly speaking, appears to denote the differential of $x^{2}$. There can be no doubt, however, which notation is the most convenient in the extension of the system to the calculus of variations, where the symbol is $\delta$; for, although the variation of a fluxion or differential may perhaps even more conveniently be expressed by $\delta \dot{x}$ than by $\delta d x$, yet the fluxion of a variation can with no convenience be expressed by $\frac{\dot{\delta}}{\delta x}$, or otherwise than by $d \delta x$. The expression of second fluxions undeveloped is also far less convenient by the Newtonian notation. Thus the fluxion of $\frac{d y}{d x}$ is sometimes required to be expressed without developement, as in the expression for the radius of curvature, where it is often expedient not to develope it in the general equation, but to find $\frac{d y}{d x}$ in terms of $x$ or $y$ before taking its fluxion; yet nothing can be more clumsy than to place a dot over the fraction, whereas $d\left(\frac{d y}{d x}\right)$ is perfectly convenient.

Several important considerations arise out of the nature and origin of these infinitesimal quantities as we have described them; and to these considerations we must now shortly advert, as they give the rules for finding the

$$
\text { C } 4
$$


fluxions or differentials of all quantities, and, conversely, lead to those for investigating or finding the fluents or integrals of fluxional or differential expressions.

A rectangle A $\mathrm{M}$ being generated by the side $\mathrm{P} M$ moving along $\mathrm{A} P$ while the side $\mathrm{N} \mathrm{M}$ moves along $\mathrm{A} N$, the movement or fluxion or differential of $\mathrm{AM}$, or of $\mathrm{A} \mathrm{P} \times \mathrm{PM}$, is $\mathrm{PS}+\mathrm{MO}$, part of the gnomon $\mathrm{P} \mathrm{S}$

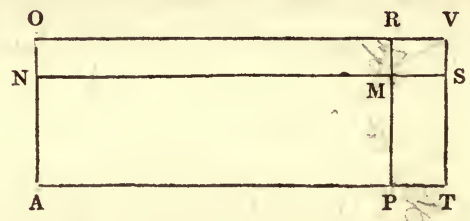

$+\mathrm{S} \mathrm{O}$, because the rectangle $\mathrm{MV}$ is evanescent compared with the other two, and is to be rejected. Therefore the differential of $\mathrm{AP} \times \mathrm{PM}=\mathrm{PM} \times \mathrm{PT}+\mathrm{NM}$ $\times \mathrm{N} \mathrm{O}$, or $\mathrm{PM} \times \mathrm{PT}+\mathrm{AP} \times \mathrm{MR}$. Calling $\mathrm{A} \mathrm{P}$ $=x$, and $\mathrm{PM}=y$, and $\mathrm{P} \mathrm{T}=d x$, and $\mathrm{MR}=d y$, we have the differential of $x y=x d y+y d x$. But if the figure be a square, and $\mathrm{AP}=\mathrm{P} \mathrm{M}$, or $x=y$, then the differential is $2 x d x$. So if we would find the differential of a parallelopiped whose sides are $x, y$, and $z$, we shall in like manner find that it is $x y d z+x z d y$ $+y z d x$; if $x=z$, then it is $2 y x d x+x^{2} d y$; and if $x=y=z$, or the figure be a cube, it is $3 x^{2} d x$. From hence, although the geometrical analogy serves us no further (as there are only three dimensions in figures), we derive by analogy the rule that the differential of $x^{m}$ is $m x^{m-1} d x$. Also there is no dimension of figure less than unity; but by the same analogy we obtain the differential of $x^{-m}$, or $\frac{1}{x^{m}}$, namely, $-m x^{-m-1} d x$, or $-\frac{m d x}{x^{m+1}}$, 
and of $\frac{x^{m}}{y^{n}}$, or $x^{m} \times y^{-n}=m x^{m-1} y^{-n} d x-n x^{m} y^{-n-1} d y$, or $=\frac{m x^{m-1} y^{n} d x-n x^{m} y^{n-1} d y}{y^{2 n}}$.

Consistently with the same principles, we may deduce this rule otherwise and more strictly. Let $x+d x$ be the quantity when increased by the differential. This multiplied by itself, or its square when completed, is $x^{2}$ $+2 x d x+(d x)^{2}$; but to have the mere increment or differential we must deduct $x^{2}$, and we must also reject $(d x)^{2}$ as evanescent compared with the function $2 x d x$, which leaves $2 x d x$ for the differential. So the cube is $x^{3}+$ $3 x^{2} d x+3 x\left(d x^{2}\right)+(d x)^{3}$, and rejecting, in like manner, we have $3 x^{2} d x$; and by the binomial theorem $(x+d x)^{m}$ is $x^{m}+m x^{m-1} d x,+\& c .+(d x)^{m}$, of which only the second term can upon the same principles be retained; that is $m x^{m-1} d x$ : And the same rules apply to the differentials of surds; so that the differential of $(x+y)^{\frac{1}{2}}$ is $\frac{d x+d y}{2 \sqrt{x+y}}$.

It also follows that the fluent or integral is a quantity such that, by taking its fluxion or differential according to the foregoing principles, you obtain the given fluxional or differential expression. Thus if we have to integrate any quantity as $x^{m} d x$, we divide by $m+1$, and increase the exponent by unity, and erase the differential quantity; so that $\frac{x^{m+1}}{m+1}$ is the integral required. But as every multiplication of any two quantities whatever gives a finite product, and every involution a finite power, while we can only divide so as to obtain a finite quotient, or extract so as to obtain a finite root, where the dividend or the power operated upon happens to be a perfect product or a perfect power; so in like 
manner we can only obtain the exact integral where the expression submitted to us is a complete differential. Thus, though such an expression as $\frac{x d x}{\sqrt{1+x^{2}}}$ is integrable, such an expression as $\frac{d x}{\sqrt{1+x^{2}}}$ is not integrable, for want of the $x$ in the numerator; and various approximations and other contrivances are resorted to in order to accomplish or, at least, approach this object, of which the methods of series, of logarithms, and circular arcs are the most frequently used. The simplest case of integration by series may be understood in examples like the last; for if the square root be extracted by a series, we may be able to integrate each term, and so by the sum of the integrals to approach the real value of the whole.

From the doctrine as now explained, and the original foundations of the method as traced above, it follows that a variety of the most important problems may be solved with ease and certainty, which by the ancient geometry could only in certain cases, or by a happy accident, be investigated. Thus the tangents of curves may be found. For as the subtangent S P : P M :: M N : T N, S P $=\frac{P M \times M N}{N T}$

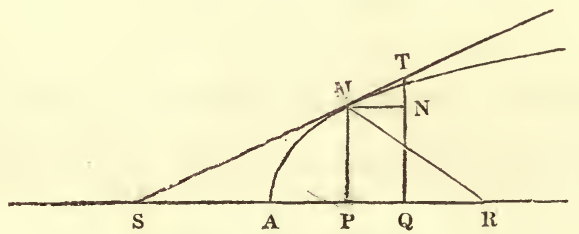

$=\frac{y d x}{d y}:$ And so the perpendicular may always be drawn; for the subnormal $\mathrm{RP}=\frac{\mathrm{P} \mathrm{M}^{2}}{\mathrm{SP}}=\frac{y^{2} d y}{y d x}=\frac{y d y}{d x}$. Therefore we have only to insert the one of these quantities in terms of the other from the equation between $x$ and $y$ (the equation to the curve), and we get the expressions for the 
subtangent and subnormal. Thus in the common parabola, whose equation is $y^{2}=a x$, the subtangent $\frac{y d x}{d y}=\frac{2 y d y}{a}$ $\times \frac{y}{d y}=\frac{2 y^{2}}{a}$ or $2 x$; and in the hyperbola, whose equation is $x y=a^{2}$, the subtangent is $-x$. So in the circle $y^{2}=2 r x-x^{2}, \frac{y d y}{d x}$ (the subnormal) $=r-x$ ( $r$ being the radius); all which we know from geometrical demonstration to be true.

Next, it is evident that when a quantity increasing has attained its maximum, it can have no further increment; or when decreasing it has attained its minimum, it can have no further decrement; consequently in such cases the differential of the quantity is equal to nothing.* Hence a ready solution is afforded of problems of maxima and minima. Thus would we know the proportion which two sides of a rectangle must have to each other, in order that, their sum being given, they may form a rectangle containing the greatest space possible; the differential of the rectangle must be put equal to nothing. Thus their sum being $=a$, the quantities are $x$ and $a-x$, and their rectangle is $a x-x^{2}$, its differential $a d x-2 x d x$, and this being put $=0$, we have $a d x=2 x d x$, or $x=\frac{a}{2}$; therefore the figure must be a square. So would we know the point of the parabola $(b-x)^{2}=a(y-c)$ where the curve comes nearest the line $b$, the ordinate $y$ must be a minimum, and $d y=0$. Now $y=\frac{(b-x)^{2}}{a}+c$, and $d y=\frac{2(b-x)}{a} \times$

* Sir I. Newton's own statement of the method is here followed. Methodus Fluxionum - Opuscula, tom. i. p. 86. edit. Geneva, 1744. It has, however, been since universally admitted that the more accurate view is to regard the change of the sign as the criterion, both as to maximum and minimum values, and as to points of contrary flexure. 
$-d x$, which being put $=0$ gives us $x=b$; or, at the extremity of the line $b$, the curve approaches the nearest; and that whatever be its parameter; for $a$ has vanished from the equation.

Again, we have seen that the ultimate ratio of the sum of all the rectangles $\mathrm{M} P \times \mathrm{P}$, contained by the ordinates and the increments of the abscissa to the curve's area $\mathrm{A} \mathrm{P} \mathrm{M}$ is that of equality; or, in other words, that the differential of a curvilinear area being the rectangle contained by the ordinate and the differential of the abscissa, or $y d x$, the integral of this, or the sum of all those small rectangles, is equal to the area. In this expression, then, let $y$ be inserted in terms of $x$, and the integral gives the area. Thus in the parabola $y=\sqrt{a x}$; therefore $d x \sqrt{a} x$ is the differential of the area, and its integral, or which is the same thing, the integral of $\frac{2 y^{2} d y}{a}$, is $\frac{2}{3} \times \frac{y^{3}}{a}$, or $\frac{2}{3} \times \frac{y^{2}}{a}$ $\times y$, that is, $\frac{2}{3} x y$, or two-thirds of the rectangle of the co ordinates; as we also know from conic sections.

Next, we have seen that the ratio of the infinitely small rectilinear sides into which a curve line may be divided (each of those small lines being the hypothenuse of a right-angled triangle, the sides of which are the differentials $\mathrm{N} \mathrm{T,} \mathrm{M} \mathrm{N} \mathrm{of} \mathrm{the} \mathrm{co-ordinates),} \mathrm{to} \mathrm{the} \mathrm{infinitely} \mathrm{small}$ portions of the curve itself is that of equality; therefore the differential of the curve is equal to the square root of the sum of the squares of the differentials of the ordinate and abscissa, and that differential is equal to $\sqrt{d x^{2}+d y^{2}}$. Hence in the circle, an arc whose cosine is $x$ and radius $r$ is equal to the integral of $\frac{r d x}{\sqrt{r^{2}-x^{2}}}$. And an arc whose cosine is $r-x$, is equal to the integral of $\frac{r d x}{\sqrt{2 r x-x^{2}}}$. 
Again, because solids may in like manner be considered as composed of infinitely thin solids or plates, one placed upon the other, their differential is the area of the surface multiplied by the differential of the axis. Thus the base of any solid generated by the revolution of a surface rectilinear or curved must be a circle, and the proportion of the radius to the circumference being taken as $r: c, y$ being the ordinate to the line bounding the vertical section, the surface will be $\frac{c y^{2}}{2 r}$ and the differential of the axis $x$ being $d x$, the differential of the solid will be $\frac{c \dot{y}^{2} d x}{2 r}$, in which $y$ in terms of $x$ being inserted from the boundary line's equation, the integral gives the solid content. Thus if the line which bounds is straight and parallel to the axis, or the solid is a cylinder, its content is the circle multiplied by the axis; and if the line is drawn to a point in the axis, or the solid is a cone, then its content is one-third of the same product, or one-third of the cylinder-well-known properties of those two figures, proved by ordinary geometry. So in like manner we find the sphere to be two-thirds of the circumscribing cylinder, the celebrated discovery of Archimedes, of which he caused the diagram to be inscribed on his tomb.

Lastly, it may in like manner be shown that the radius of the osculating circle at any point of any curve, that is, the circle touching it at such point, and having the same curvature with it at that point, is equal to $\frac{\left(d x^{2}+d y^{2}\right)^{\frac{3}{2}}}{ \pm d x^{2} \times d\left(\frac{d y}{d x}\right)}$, where $d y$ being found in terms of $x$, the differential of $\frac{d y}{d x}$ is to be taken, so that there will in the result in each case be no differential at all. 
Thus in the parabola $y^{2}=2 \alpha x$, the radius of curvature is $=\sqrt{\frac{2 x+a}{a}} \times(2 x+a)$.

In all these operations, however, it must be observed, that as constant or invariable quantities have no differentials, so when we reverse the operation and find integrals from given differential expressions, we never can tell whether a constant must not be added in order to complete that quantity, by taking whose differential the given expression was originally obtained. The determining of this constant quantity, and the finding whether there be any or not, depends upon the particular conditions of each problem. It is always added as a matter of course. Thus when we integrate $d x+d y$, we cannot tell whether this quantity arose from taking the differentials of $x$ and $y$ only, or from taking the differential of $x+y+c$; and it must depend upon the nature of the question whether $c$ is to be added to the integral or no; and if to be added, how it shall be ascertained.

Having explained this important method of investigation, by the help of which Newton was enabled to make his greatest mathematical discoveries, and by the principles of which he demonstrates them in the Principia, it only remains, before proceeding to the analysis of those discoveries, that we should remark the preference which he gives to the geometrical methods, improved and adapted to his purpose by the doctrine of Prime and Ultimate ratios. He uses this doctrine similar in principle to, and the foundation of, the noble and refined calculus which we have been considering; but he does not at all employ that calculus.

The First book treats of the motion of bodies with- 
out regard to the resistance of the medium that fills the space in which they move; and it is principally devoted to the consideration of motions in orbits determined by centripetal forces, and to examining the attraction of bodies. The Second book treats of the resistance of fluids chiefly as affecting the motions of bodies that move in them. The Third book contains the application of the principles thus established to the motions, attractions, and figures of the heavenly bodies.

I.

The fundamental proposition, as it may justly be termed, of the whole system, is one which Newton's predecessors may be said to have nearly reached; which Kepler, had he been more inclined to trust demonstration than empirical observation, probably would have attained; and which Galileo would certainly have discovered had he contemplated the facts discovered by Kepler, particularly his second law*: The proposition is this. If a body is driven by any single impulse or force of projection, and is also drawn continually by another force so as to revolve round a fixed centre, the radius vector, or line drawn from the body to that centre, describes areas which are in the same fixed plane, and are always proportional to the times of the body's motion; and conversely, if any body which moves in any curve described in a plane so that the radius vector to a point either fixed or moving uniformly in a straight line, describes areas proportional to the times of the body's motion, that body is acted on by a centripetal force tending towards and drawing it to the point.

To prove this, we have to consider that if a body moves equably on in a straight line, the areas or triangles

* See the historical notice above respecting this second law, viz., that the planets describe areas proportional to the times by their radii vectores. 
which are described by a line drawn from it to any point are proportional to the portions of the straight line through which the body moves, (that is to the time, since, moving equably, it moves through equal spaces in equal times,) because those triangles, having the same altitude, are to one another in the proportions of their bases. S being the point and $\mathrm{AO}$ the line of motion, $\mathrm{SAB}$ is to $\mathrm{SB} c$ as $\mathrm{AB}$ to $\mathrm{B} c$. If then at $\mathrm{B}$ a force acts in the line $\mathrm{SB}$, drawing the body towards $\mathrm{S}$, it will move in the diagonal $\mathrm{BC}$ of a parallelogram of which the sides are $\mathrm{B} c$ and $\mathrm{BV}$, the line through which the deflecting force

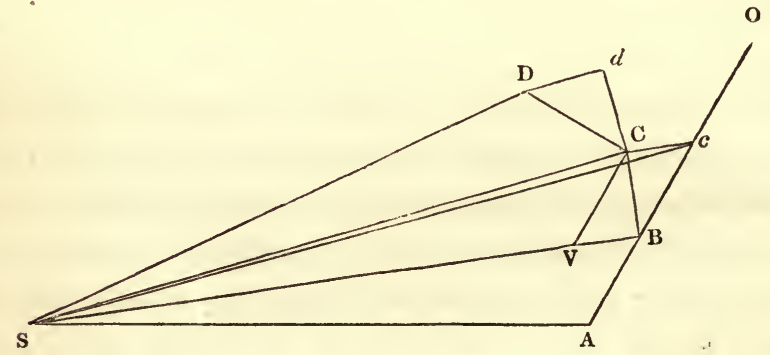

would make it move if the motion caused by the other force ceased. $\mathrm{C} c$ therefore is parallel to $\mathrm{VB}$, and the triangle $\mathrm{SBC}$ is equal to the triangle $\mathrm{SB} c$; consequently the motion through $\mathrm{AB}$ and $\mathrm{BC}$, or the times, are as the two triangles $\mathrm{SAB}$ and $\mathrm{SBC}$ : and so it may be proved if the force acting towards $\mathrm{S}$ again deflects the body at $\mathrm{C}$, making it move in the diagonal CD. If, now, instead of this deflecting force acting at intervals $\mathrm{A}, \mathrm{B}, \mathrm{C}$, it acts at every instant, the intervals of time become less than any assignable time, and then the spaces $\mathrm{AB}, \mathrm{BC}, \mathrm{CD}$ will become also indefinitely small and numerous, and they will form a curve line; and the straight lines drawn from any part of that curve to $\mathrm{S}$ will describe curvilinear areas, as the body moves in the curve $\mathrm{ABCD}$, those areas being proportional to the times.-So conversely, if the triangles 
$\mathrm{SB} c$ and $\mathrm{SBC}$ are equal, they are between the same parallels, and $c \mathrm{C}$ is parallel to $\mathrm{SB}$, and $\mathrm{D} d$ to $\mathrm{SC}$; consequently the force which deflects acts in the lines SB and SC, or towards the point $\mathrm{S}$. - It is equally manifest that the direction of the lines $\mathrm{B} c, \mathrm{C} d$, from which the centripetal force deflects the body, is that of tangents to the curve which the body describes, and that consequently the velocity of the body is in any given point inversely proportional to the perpendicular drawn from the centre to the tangent; the areas of the triangles whose bases are equal, being in the proportion of their altitude, that is, of those perpendiculars, and those areas being by the proposition, proportional to the times.

There are several other corollaries to this important proposition which deserve particular attention. B $c$ and $\mathrm{D} e$ are tangents to the curve at $\mathrm{B}$ and $\mathrm{D}$ respectively;

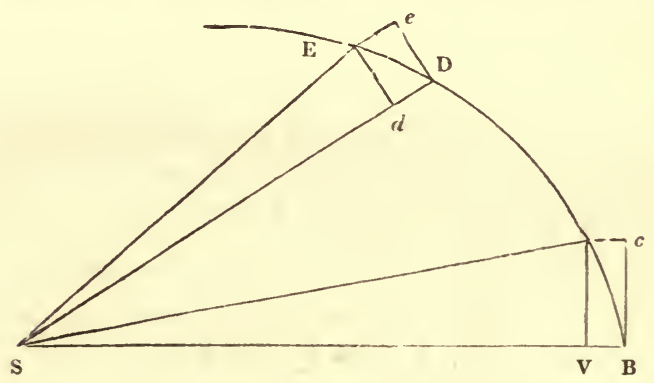

$\mathrm{B} \mathrm{C}$ and $\mathrm{D} \mathrm{E}$ the arcs described in a given time; $\mathrm{C} c$ and $\mathrm{E} e$ lines parallel to the radii vectores $\mathrm{S} \mathrm{B}$ and $\mathrm{S} \mathrm{D}$ respectively; and $\mathrm{C} \mathrm{V}, \mathrm{E} d$ parallel to the tangents. The centripetal forces at $\mathrm{B}$ and $\mathrm{D}$ must be in the proportion of $\mathrm{V} \mathrm{B}$ and $d \mathrm{D}$ (being the other sides of the parallelograms of forces) if the arcs are evanescent, so as to coincide with the diagonals of the parallelograms $\mathrm{V} c$ and $d e$. Hence the centripetal forces in $\mathrm{B}$ and $\mathrm{D}$ are as the versed sines 
of the evanescent arcs; and the same holds true if instead of two ares in the same curve, we take two arcs in different but similar curves.*

From these propositions another follows plainly, and its consequences are most extensive and important. If two or more bodies move in circular orbits (or trajectories) with an equable motion, they are retained in those paths by forces tending towards the centres of the circles; and those forces are in the direct proportion of the squares of the arcs described in a given time, and in the inverse proportion of the radii of the circles.

First of all it is plain, by the fundamental proposition, that the forces tend to the centres $\mathrm{S}, s$, because the sectors $\mathrm{A} \mathrm{SB}$ and $\mathrm{PBS}$ being as the $\operatorname{arcs} \mathrm{AB}, \mathrm{B} \mathrm{P}$, and the sectors $a s b, p b s$, as the $\operatorname{arcs} a b, b p$, which arcs being all as the times, the areas are proportional to those times of describing them, and therefore $\mathrm{S}$ and $s$ are the centres of the deflecting forces. Then, drawing the tangents A C, $a c$, and completing the parallelograms $\mathrm{D} \mathrm{C}, d c$, the diagonals of which coincide with the evanescent $\operatorname{arcs} \mathrm{A} B, a b$, we have the centripetal forces in $\mathrm{A}$ and $a$, as the versed sines $\mathrm{A} \mathrm{D}, a d$. But because $\mathrm{A} \mathrm{B} \mathrm{P} \mathrm{and} a b p$ are right angles (by the property of the circle), the triangles $\mathrm{ADB}$, $\mathrm{A} \mathrm{P} \mathrm{B}$, and $a d b, a p b$, are respectively similar to one another. Wherefore $\mathrm{A} D: \mathrm{AB}:: \mathrm{AB}: \mathrm{A} \mathrm{P}$ and $\mathrm{AD}$ $=\frac{\mathrm{AB}}{\mathrm{AP}}$; and in like manner $a d=\frac{a b^{2}}{a p}$, or, as the evanescent arcs coincide with the chords, $\mathrm{AD}=\operatorname{arc} \frac{\mathrm{A} \mathrm{B}^{2}}{\mathrm{AP}}$ and $a d=\operatorname{arc} \frac{a b^{2}}{a p}$. Now these are the properties of any arcs described in equal times; and the diameters are in the pro-

* If $\mathrm{BC}, \mathrm{DE}$, are bisected, the proportion is found with the halves of $\mathrm{V} \mathrm{B}, \mathrm{Dd}$; and that is the same proportion with the whole versed sines. 
portion of the radii; therefore the centripetal forces are directly as the squares of the arcs, and inversely as the radii.
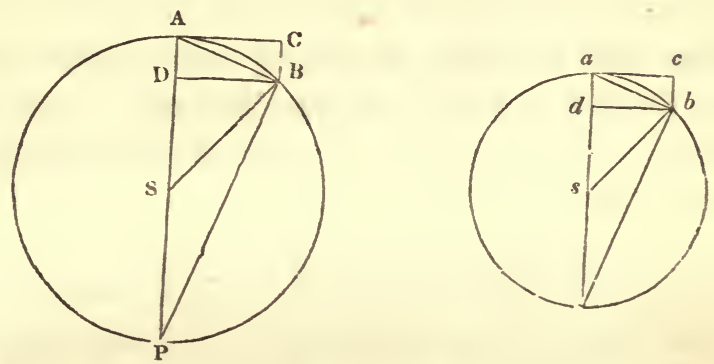

It is difficult to imagine a proposition more fruitful in consequences than this; and therefore it has been demonstrated with adequate fulness.

In the first place, the ares described being as the velocities, if $\mathrm{F}, f$ are the centripetal forces, and $\mathrm{V}, v$ the velocities, and $\mathrm{R}, r$ the radii, $\mathrm{F}: f:: \mathrm{V}^{2}: v^{2}$; and also $:: r: \mathrm{R}$; or $\mathrm{F}:$

$f:: \frac{\mathrm{V}^{2}}{\mathrm{R}}: \frac{v^{2}}{r}$. Now as in the circle $\mathrm{V}$ and $\mathrm{R}, v$ and $r$ are both constant quantities, the centripetal force is itself constant, which retains a body by deflecting it towards the centre of the circle.

Secondly. The times in which the whole circles are described (called the periodic times) are as the total circumferences or peripheries; $\mathrm{T}: t:: \mathrm{P}: p:$ But the peripheries are as the radii or $:: \mathrm{R}: r$. Therefore $\mathrm{T}:$

$t:: \mathrm{R}: r ;$ also $\mathrm{V}: v:: \frac{\mathrm{P}}{\mathrm{T}}: \frac{p}{t}$, therefore inversely as the radii, or $\mathrm{T}: t:: \frac{\mathrm{R}}{\overline{\mathrm{V}}}: \frac{r}{v}$ and $\mathrm{V}^{2}: v^{2}:: \frac{\mathrm{R}^{2}}{\mathrm{~T}^{2}}: \frac{r^{2}}{t^{2}}$. But the centripetal forces $\mathrm{F}: f:: \frac{\mathrm{V}^{2}}{\mathrm{R}}: \frac{v^{2}}{r}$; substituting for the 
ratio of $\mathrm{V}^{2}: v^{2}$, its equal the ratio of $\frac{\mathrm{R}^{2}}{\mathrm{~T}^{2}}: \frac{r^{2}}{t^{2}}, \mathrm{~F}: f:$ : $\frac{\mathrm{R}}{\mathrm{T}^{2}}: \frac{r}{t^{2}}$; or the centripetal forces are directly as the distances and inversely as the squares of the periodic times; the forces being as the distances if the times are equal; and the times being equal if the forces are as the distances. - It also follows that if the periodic times are as the distances, then $\mathrm{F}: f:: \frac{\mathrm{R}}{\mathrm{R}^{2}}: \frac{r}{r^{2}}$; that is, $:: \frac{1}{\mathbf{R}}: \frac{1}{r}$, or inversely as the distances. - In like manner if the periodic times are in proportion to any power $n$, of the distance, or $\mathrm{T}: t:: \mathrm{R}^{n}: r^{n}$, we shall have $\mathrm{T}^{2}: t^{2}:: \mathrm{R}^{2 n}: r^{2 n}$ and $\mathrm{F}: f:: \frac{\mathrm{R}}{\mathrm{R}^{2 n}}: \frac{r}{r^{2 n}}$; that is $\therefore \frac{1}{\mathrm{R}^{2 n-1}}: \frac{1}{r^{2 n-1}}$; and conversely if the centripetal force is in the inverse ratio of the $(2 n-1)^{\text {th }}$ power of the distance, the periodic time is as the $n^{\text {th }}$ power of that distance.-Likewise, as the velocities of the bodies in their orbits or $\mathrm{V}: v:: \frac{\mathrm{R}}{\mathrm{T}}: \frac{r}{t}$, if we make $\mathrm{T}: t:: \mathrm{R}^{n}: r^{n}$, then $\mathrm{V}: v:: \frac{\mathrm{R}}{\mathrm{R}^{n}}: \frac{r}{r^{n}}$, or $:: \frac{1}{\mathrm{R}^{n-1}}: \frac{1}{r^{n-1}}$. Thus, suppose $n$ is equal to $\frac{3}{2}$ we have for the velocities $\mathrm{V}: v$ $:: \frac{1}{\sqrt{R}}: \frac{1}{\sqrt{r}}$, or they are in the inverse subduplicate proportion of the distances; and for the centripetal forces we have $\mathrm{F}: f:: \frac{1}{\mathrm{R}^{3-1}}: \frac{1}{r^{3-1}}:: \frac{1}{\mathrm{R}^{2}}: \frac{1}{r^{2}} ;$ or the attraction to the centre is inversely as the square of the distance. 
Now if $n=\frac{3}{2}, \mathrm{~T}: t:: \mathrm{R}^{\frac{3}{2}}: r^{\frac{3}{2}}$, or $\mathrm{T}^{2}: t^{2}:: \mathrm{R}^{3}: r^{3} ;$ in other words the squares of the periodic times are as the cubes of the distances from the centre, which is the law discovered by Kepler from observation actually to prevail in the case of the planets. And as he also showed from observation that they describe equal areas in equal times by their radii vectores drawn to the sun, it follows from the fundamental proposition, first, that they are deflected from the tangents of their orbits by a power tending towards the sun; and then it follows, secondly, from the last deduction respecting it, (namely, the proportion of $\mathrm{F}: f:: \frac{1}{\mathrm{R}^{2}}: \frac{1}{r^{2}}$,) that this central force acts inversely as the squares of the distances, always supposing the bodies to move in circular orbits, to which our demonstration has hitherto been confined.*

The extension, however, of the same important proposition to the motion of bodies in other curves is easily made, that is to the motion of bodies in different parts of the same curve or in curves which are similar. For in evanescent portions of the same curve, the osculating circle, or circle which has the same curvature at any point, coincides with the curve at that point; and if a line is drawn to the extremity of that circle's diameter, A M B and $a m b$ may be considered as triangles; and as they are right angled at $\mathrm{M}$ and $m, \mathrm{~A} \mathrm{M}^{2}$ is equal to $\mathrm{A} \mathrm{P} \times \mathrm{AB}$ and $a m^{2}$ to $a p \times a b$; and where the curvature is the same as in corresponding points of similar curves, those squares are proportional to the lines $\mathrm{A} \mathrm{P}$, or $a p$; or those versed

* We shall afterwards show, from other considerations, that this sesquiplicate proportion only holds true on the supposition of the bodies all moving without exerting any action on each other, when we come to consider Laplace's theorems on elliptical motion. 
sines of the arcs $\mathrm{A} \mathbf{M}$ and $a m$ are proportional to the squares of the small ares. Hence if the distances of two
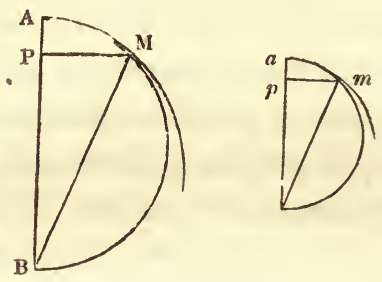

bodies from their respective centres of force be $\mathrm{D}, d$, the deflecting force in any points $\mathrm{A}$ and $a$ being as the versed sines, those forces are as $\mathrm{A} \mathrm{M}^{2}: a m^{2}$; and from hence follows generally in all curves, that which has been demonstrated respecting motion in circular orbits.

The planets then and their satellites being known by Kepler's laws to move in elliptical orbits, and to describe round the sun in one focus areas proportional to the times by their radii vectores drawn to that focus, and it being further found by those laws that the squares of their periodic times are as the cubes of the mean distances from the focus, they are by these propositions of Sir Isaac Newton which we have been considering, shown to be deflected from the tangent of their orbit, and retained in their paths, by a force acting inversely as the squares of the distances from the centre of motion.

But another important corollary is also derived from the same proposition. If the projectile or tangential force in the direction A T ceases (next figure), the body, instead of moving in any $\operatorname{arc} \mathbf{A} \mathrm{N}$, is drawn by the same centripetal force in the straight line A S. Let A $n$ be the part of $\mathrm{A} \mathrm{S}$, through which the body falls by the force of gravity, in the same time that it would take to describe the are $\mathbf{A} \mathbf{N}$. Let $\mathbf{A} \mathbf{M}$ be the infinitely small 
are described in an instant; and $\mathrm{A} \mathrm{P}$ its versed sine. It was before shown, in the corollaries to the first proposition, that the centripetal force in $A$ is as $A P$, and the body would move by that force through A P, in the same time in which it describes the arc A M. Now the force of gravity being one which operates like the centripetal force at every instant, and uniformly accelerates the descending body, the spaces fallen through will be as the squares of the times. Therefore, if $\mathbf{A} n$ is the space through which the body falls in the same time that it

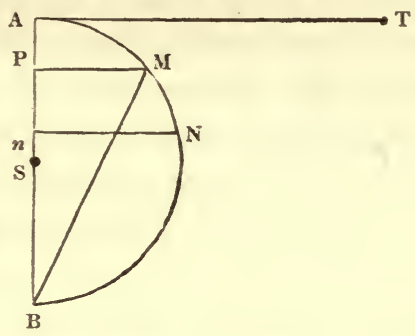

would describe $\mathrm{A} \mathrm{N}, \mathrm{A} \mathrm{P}$ is to $\mathrm{A} n$ as the square of the time taken to describe $\mathbf{A} \mathbf{M}$ to the square of the time of describing $\mathrm{A} \mathrm{N}$, or as $\mathrm{A} \mathrm{M}^{2}: \mathrm{A} \mathrm{N} \mathrm{N}^{2}$, the motion being uniform in the circular arc. But $\mathbf{A} \mathbf{M}$, the nascent arc, is equal to its chord, and $\mathrm{A}$ M B being a right angled triangle as well as A P M, A B : A M :: A M : A P and $\mathrm{A} P=\frac{\mathrm{A} \mathrm{M}^{2}}{\mathrm{~A} \mathrm{~B}}$. Substituting this in the former proportion, we have $\frac{\mathrm{AM}^{2}}{\mathrm{AB}}: \mathrm{A} n:: \mathrm{A} \mathrm{M}^{2}: \mathrm{A} \mathrm{N}^{2}$, or $\mathrm{A} n: \mathrm{A} \mathrm{N}^{2}$ $: \frac{\mathrm{AM}^{2}}{\mathrm{AB}}: \mathrm{A} \mathrm{M}^{2}$, that is $:: 1: \mathrm{AB}$. Therefore $\mathrm{A} \mathrm{N}^{2}$ $=\mathrm{A} n \times \mathrm{A} \mathrm{B}$, or the arc described, is a mean proportional between the diameter of the orbit, and the space through which the body would fall by gravity alone, in the same time in which it describes the arc. 
Now let A M N B represent the orbit of the moon; A $\mathrm{N}$ the arc described by her in a minute. Her whole periodic time is found to be 27 days 7 hours and $43 \mathrm{mi}$ nutes, or 39,343 minutes; consequently $\mathrm{A} \mathrm{N}: 2 \mathrm{~A} \mathrm{~N} \mathrm{~B}$ $:: 1: 39,343$.

But the mean distance of the moon from the earth is about 30 diameters of the earth, and the diameter of her orbit, 60 of those diameters; and a great circle of the earth being about 131,630,572 feet, the circumference of the moon's orbit must be 60 times that length, or $7,897,834,320$, which being divided by 39,343 (the number of minutes in her periodic time), gives for the arc A $\mathrm{N}$ described in one minute 200,743, of which the square is $40,297,752,049$, or $\mathrm{A} \mathrm{N}^{2}$, which (by the proposition last demonstrated) being divided by the diameter $\mathrm{A} \mathrm{B}$ gives $\mathrm{A} n$. But the diameter being to the orbit as $1: 3.14159$ nearly, it is equal to about $2,513,960,866$. Therefore A $n=16.02958$, or 16 feet, and about the third of an inch. But the force which deflects the moon from the tangent of her orbit, has been shown to act inversely as the square of the distance; therefore she would move $60 \times 60$ times the same space in a minute at the surface of the earth. But if she moved through so much in a minute, she would in a second move through so much less in the proportion of the squares of those two times, as has been before shown. Wherefore she would in a second move through a space equal to $16 \frac{1}{3}$. nearly (16.02958). But it is found by experiments frequently made, and among others by that of the pendulum *, that a

* It is found that a pendulum, vibrating seconds, is about the length of 3 feet $3 \frac{1}{5}$ inches in this latitude; and the space through which a body falls in a second is to half this length as the square of the circumference of a circle to that of the diameter, or as $9.8695: 1$, and that is the proportion of the half of 3 feet $3 \frac{1}{5}$ inches to somewhat more than 16 feet. 
body falls about this space in one second upon the surface of the earth. Therefore the force which deflects the moon from the tangent of her orbit, is of the same amount, and acts in the same direction, and follows the same proportions to the time that gravity does. But if the moon is drawn by any other force, she must also be drawn by gravity; and as that other force makes her move towards the earth 16 feet $\frac{1}{3}$ inch, and gravity would make her move as much, her motion would therefore be 32 feet $\frac{2}{3}$ inch in a second at the earth's surface, or as much in a minute in her orbit; and her velocity in her orbit would therefore be double of what it is, or the lunar month would be less than 13 days and 16 hours. It is, therefore, impossible that she can be drawn by any other force, except her gravity, towards the earth.*

Such is the important conclusion to which we are led from this proposition, that the centripetal forces are as the squares of the arcs described directly, and as the distances inversely. The great discovery of the law of the universe, therefore. is unfolded in the very beginning of the Principia. But the rest of the work is occupied with tracing the various consequences of that law, and first of all in treating generally of the laws of curvilinear motion. The demonstration of the moon's deflection has been now anticipated and expounded from the Third Book, where it is treated with even more than the author's accustomed conciseness. But there seemed good ground for this anticipation, inasmuch as the Scholium to the Fourth Proposition refers in general terms to the con-

* The proposition may be demonstrated by means of the Prop. XXXVI. of Book I., as well as by means of the proposition of which we have now been tracing the consequences (Prop. IV). But in truth the latter theorem gives a construction of the former problem (Prop. XXXVI.), and from it may be deduced both that and Prop. XXXV. 
nexion between its corollaries, and the Theory of Gravitation.

The versed sine of the half of any evanescent arc (or sagitta of the arc) of a curve in which a body revolves, was proved to be as the centripetal force, and as the square of the times; or as $\mathrm{F} \times \mathrm{T}^{2}$. Therefore the force $\mathrm{F}$ is directly as the versed sine, and inversely as the square of the time. From this it follows that the central force may be measured in several ways. The arc being $\mathrm{Q} \mathrm{C}$, we are to measure the central force in its middle point $\mathrm{P}$. Then the areas being as the times; twice the triangle $\mathrm{S} \mathrm{P} Q$, or $\mathrm{Q} \mathrm{L} \times \mathrm{S} \mathrm{P}$ is as $\mathrm{T}$ in

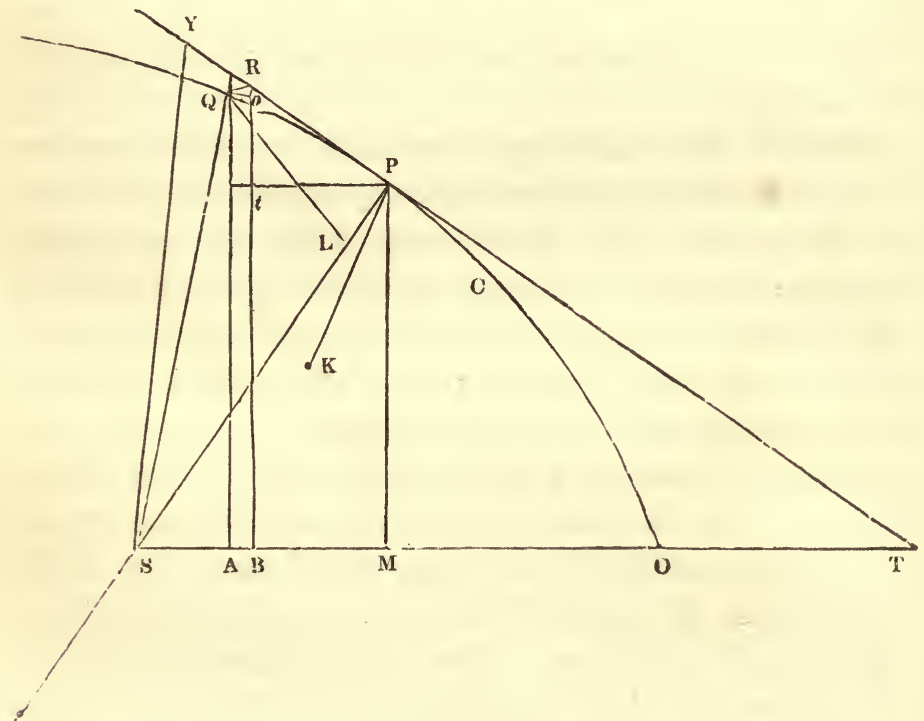

the last expression; and, therefore, $\mathrm{Q} \mathrm{R}$ being parallel to $\mathrm{LP}$, the central force at $\mathrm{P}$ is as $\frac{\mathrm{QR}}{\mathrm{SP}^{2} \times \mathrm{LQ}^{2}}{ }^{\circ}$ So if $S Y$ be the perpendicular upon the tangent $P Y$, because $P R$ and the arc $P Q$, evanescent, coincide, twice the triangle $\mathrm{SPQ}$ is equal to $\mathrm{S} \mathrm{Y} \times \mathrm{QP}$; and the 
central force in $\mathrm{P}$ is as $\frac{\mathrm{QR}}{\mathrm{SY}^{2} \times \mathrm{QP}^{2}}$. Lastly, if the revolution be in a circle, or in a curve having at $\mathrm{P}$ the same curvature with a circle whose chord passes from that point through $\mathrm{S}$ to $\mathrm{V}$, then the measure of the central force will be $\frac{1}{\mathrm{SY}^{2} \times \mathrm{PV}^{-}}-\mathrm{By}$ finding the value of those solids in any given curve, we can determine the centripetal force in terms of the radius vector SP; that is, we can find the proportion which the force must bear to the distance, in order to retain the body in the given orbit or trajectory; and conversely, the force being given, we can determine the trajectory's form.

This proposition, then, with its corollaries, is the foundation of all the doctrine of centripetal forces, whether direct or inverse; that is, whether we regard the method of finding, from the given orbit, the force and its proportion to the distance, or the method of finding the orbit from the given force. We must, therefore, state it more in detail, and in the analytical manner, Sir Isaac Newton having delivered it synthetically, geometrically, and with the utmost brevity.

- It may be reduced to five kinds of formulæ.

1. If the central force in two similar orbits be called $\mathrm{F}$ and $f$, the times $\mathrm{T}$ and $t$, the versed sines of half the $\operatorname{arcs} \mathrm{S}$ and $s$, 一then $\mathrm{F}: f:: \frac{\mathrm{S}}{\mathrm{T}^{2}}: \frac{s}{t^{2}}$; and generally $\mathrm{F}$ is as $\frac{\mathrm{S}}{\mathrm{T}^{2}}$.

2. But draw $S P$ to any given point of the orbit in the middle of an infinitely small arc Q C. Let $\mathrm{T} P$ touch the curve in $\mathrm{P}$, draw the perpendicular $\mathrm{S} \mathrm{Y}$ from the centre of forces $\mathrm{S}$ to $\mathrm{P} T$ produced, draw $\mathrm{S} Q$ infinitely near $\mathrm{SP}$, and $\mathrm{Q} R$ parallel to $\mathrm{S} P, \mathrm{Q} o$ and $\mathrm{R} o$ 
parallel to the co-ordinates $\mathrm{SM}, \mathrm{M}$ P. Then $\mathrm{P}$ being the middle of the arc, twice the triangle $\mathrm{S} P \mathrm{Q}$ is proportional to the time in which $\mathrm{C} Q$ is described. Therefore $\mathrm{Q} P \times \mathrm{SY}$ or $\mathrm{QL} \times \mathrm{PS}$ is proportional to the time; and $Q R$ is the versed sine of $\frac{C Q}{2}$, therefore $\mathrm{F}$ as $\frac{\mathrm{S}}{\mathrm{T}^{2}}$ becomes $\mathrm{F}$ as $\frac{\mathrm{QR}}{\mathrm{LQ}^{2} \times \mathrm{SP}^{2}}$; and if $\mathrm{S} \mathrm{M}=x$, M P $=y$, and because the similar triangles $\mathrm{QR} o$ and $\mathrm{S} M \mathrm{P}$ give $\mathrm{QR}=\frac{\mathrm{Q} o \times \mathrm{SP}}{\mathrm{SM}}$, and because $\mathrm{A} \mathrm{M}$ being the first differential of $\mathrm{S} \mathrm{M}, o \mathrm{Q}$ is its second differential (negatively), therefore $\mathrm{Q} \mathrm{R}=\frac{-d^{2} x \times \sqrt{x^{2}+y^{2}}}{x}$ (taken with reference to $d t$ constant), and $\mathrm{F}$ is as $\frac{-d^{2} x \sqrt{x^{2}+y^{2}}}{x \times \mathrm{L} \mathrm{Q}^{2} \times\left(x^{2}+y^{2}\right)}$. But $\mathrm{L} \mathrm{Q}^{2}=\mathrm{QP}^{2}-\mathrm{L}^{2}$ and $\mathrm{L} \mathrm{P}$ is the differential of $\mathrm{S} \mathrm{P}$ or $\sqrt{x^{2}+y^{2}}$. Therefore $\mathrm{L} \mathrm{Q}^{2}=\frac{(x d y-y d x)^{2}}{x^{2}+y^{2}}=\frac{y^{4}\left(d \frac{x}{y}\right)^{2}}{x^{2}+y^{2}}$, and $\mathrm{F}$ is as $\frac{-d^{2} x \sqrt{x^{2}+y^{2}}}{x y^{4}\left(d \frac{x}{y}\right)^{2}}$.

But as the differential of the time ( $\mathrm{Q} Q \times \mathrm{P} S$ ) may be made constant, $\mathrm{Q} R$ will represent the centripetal force; and that force itself will therefore be as $-\frac{d^{2} x \sqrt{x^{2}+y^{2}}}{x}$, * taken with reference to $d t$ constant.

* Of these expressions, although I have sometimes found this, which was first given by Herrman, servieeable, I generally prefer the two, which are in truth one, given under the next heads. But the expression first given $-\frac{d^{2} x \sqrt{x^{2}+y^{2}}}{x y^{4}\left(d \frac{x}{y}\right)^{2}}$ is without integration an useful one. 
3. The rectangle $\mathrm{S} Y \times \mathrm{Q} P$ being equal to $\mathrm{Q} \mathrm{L} \times \mathrm{S} \mathrm{P}$ and $\mathrm{S} \mathrm{Y}=\frac{y d x-x d y}{\sqrt{d x^{2}+d y^{2}}}$, we have $\mathrm{F}$ as

$\frac{\mathrm{QR}}{\mathrm{S}^{2} \times \mathrm{QP}^{2}}=\frac{\mathrm{QR}}{(y d x-x d y)^{2}}=\frac{\mathrm{QR}}{y^{4}\left(d \frac{x}{y}\right)^{2}}$.

4. Because $\mathrm{F}=\frac{\mathrm{QR}}{\mathrm{SY}^{2} \times \mathrm{QP}^{2}}$ and $\frac{\mathrm{QP}^{2}}{\mathrm{QR}}$ is equal to the chord $\mathrm{P} \mathrm{V}$ of the circle, which has the same curvature with $\mathrm{Q} P \mathrm{O}$ in $\mathrm{P}$, and whose centre is $\mathrm{K}$ (and because $\mathrm{Q} \mathrm{P}^{2}=\mathrm{QR} \times \mathrm{P} \mathrm{V}$ by the nature of the circle and the equality of the evanescent arc $\mathrm{Q} P$ with its sine, and thus $\mathrm{PV}=\frac{\mathrm{Q} \mathrm{P}^{2}}{\mathrm{QR}}$, - therefore $\left.\frac{\mathrm{QR}}{\mathrm{Q} \mathrm{P}^{2}}=\frac{1}{\mathrm{PV}}\right), \mathrm{F}$ is as $\frac{1}{\mathrm{SY}^{2} \times \mathrm{PV}^{*}}$. In like manner if the velocity, which is inversely as $\mathrm{SY}$, be called $v, \mathrm{~F}$ is as $\frac{v^{2}}{\mathrm{PV}}$. Now the chord of the osculating circle is to twice the perpendicular $\mathrm{S} Y$ as the differential of S P to the differential of the perpendicular; and calling $\mathrm{S} \mathrm{P}$ the radius vector $r$, and $\mathrm{S} \mathrm{Y}=p$, we have $\mathrm{PV}=\frac{2 p d r}{d p}$, and $\mathrm{F}$ is as $\frac{d p}{2 p^{3} d r}$; and also $\mathrm{F}$ is as $\frac{v^{2} d p}{2 d r}$. In these formulas, substituting for $p$ and $r$ their values in terms of $x$ and $y$, we obtain a mean of estimating the force as proportioned to $r$, which is $\sqrt{x^{2}+y^{2}}$.

5. The last article affords, perhaps, the most obvious methods of arriving at central forces, both directly and inversely. Although the quantities become involved and embarrassing in the above general expressions for all curves, yet in any given curve the substitutions can more easily be made. A chief recommendation of these expres- 
sions is, that they involve no second differentials, nor any but the first powers of any differentials. But it may be proper to add other formulas which have been given, and one of which, at least, is more convenient than any of the rest.

One expression for the centrifugal force (and one sometimes erroneously given for the centripetal) $*$ is $\frac{d s^{2}}{2 \mathrm{R}}, s$ being the length of the curve and $R$ the radius of curvature. This gives the ready means of working if that radius is known. But its general expression involves second differentials, the usual formula for it being $\frac{d s^{3}}{d x^{2} \times d\left(\frac{d y}{d x}\right)}$; consequently we must first find $\frac{d y}{d x}=\mathrm{X}$ (a function of $x$ ), and then there are only first differentials.

Another for this radius of curvature is $\frac{d s^{2}}{\sqrt{\left(d^{2} y\right)^{2}+\left(d^{2} x\right)^{2}}}$, and this is used by Laplace; and another is $\frac{r d r}{d p}$, which, with other valuable formulas, is to be obtained from Maclaurin's Fluxions. But the formula generally ascribed to John Bernouilli (Mém. Acad. des Sciences, 1710), is, perhaps, the most elegant of any, $\mathrm{F}=\frac{r}{2 \cdot p^{3} \times \mathrm{R}}$; and this results from substituting $2 \mathrm{R}$ for its value $\frac{2 r d r}{d p}$, in the equation to $\mathrm{F}$, deduced above from Newton's formula, namely, $\mathrm{F}=\frac{d p}{2 p^{3} d r}$.

* This error appears to have arisen from taking the case where the radius of curvature and radius veetor coincide, that is, the case of the circle, in which the centrifugal and centripetal forces are the same. - See Mrs. Somerville's truly admirable work on the Mec. Cel., where the error manifestly arises from this circumstance. 
But the proposition is so important, that it may be well to prove it, and to show that it is almost in terms involved in the third corollary to Prop. VI. Book I. of the Principia. - By that corollary $\mathrm{F}=\frac{1}{p^{2} \cdot \mathrm{C}}$ (C being the osculating circle's chord which passes through the centre of forces). But drawing S Y, the perpendicular to the tangent, and P C F through the centre of the

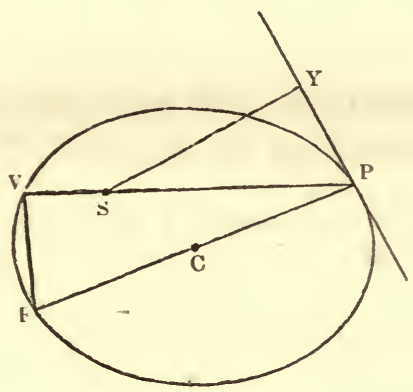

circle, and joining $\mathrm{VF}$, which is, therefore, parallel to $\mathrm{Y}$ P, we have V P : P F :: S Y : S P or C : $2 \mathrm{R}:: p: r$ and $\mathrm{C}=\frac{2 \mathrm{R} \cdot p}{r}$, which substituted for $\mathrm{C}$ in the above equation, gives $\mathrm{F}=\frac{r}{2 \mathrm{P}^{3} \cdot \mathrm{R}}$.

It is remarkable that the circumstance of this formula being thus involved in that of Sir Isaac Newton seems never to have been observed by Keill, who, in the Philosophical Transactions, xxvi. 74, gives a demonstration of it much more roundabout, and as of a theorem which Demoivre had communicated to him, adding, that Demoivre also informed him of Sir Isaac Newton having invented a similar method before. In fact, he had, above 20 years before, given it in substance, though not in express terms, in the Sixth Proposition, the addition of two lines to which would at once have led to this formula. But, again, when John 
Bernouilli, two years afterwards, wrote his letter to Herrman (Mém. Acad. des Sciences, 1710), he gives it as his own discovery, and as such it has generally been treated, with what reason we have just seen. $\mathrm{He}$ is at much pains to state, p. 529., that he had sent it in a letter to Demoivre in February, 1706 ; but the Principia had been published nineteen years before. Herrman, in his Phoronomia, erroneously considers the expression as discovered by Demoivre, Grandi, and Bernouilli. (Lib. I. Prop. XXII.)

In all these cases $p$ is to be found first, and the expression for it (because, pp. 42, 43., T P : P M :: T S: S Y and $\mathrm{T} \mathrm{S}=\frac{y d x-x d y}{d y}$, and $\left.\mathrm{P} \mathrm{T}=\frac{y}{d y} \sqrt{d y^{2}+d x^{2}}\right)$ is $p$ $=\mathrm{S} \mathrm{Y}=\frac{y d x-x d y}{\sqrt{d y^{2}+d x^{2}}}=\frac{y^{2} d \frac{x}{y}}{\sqrt{d y^{2}+d x^{2}}}$. Also $r=\mathrm{S} \mathrm{P}$ $=\sqrt{x^{2}+y^{2}}$. Then the radius of curvature $\mathbf{R}=$ $\frac{\left(d x^{2}+d y^{2}\right)^{3}}{d x^{2} \times d \mathrm{X}}$ (X being $\frac{d y}{d x}$ in terms of $x$, and having no differential in it when the substitution for $d y$ is made). Therefore, the expression for the centripetal force becomes $\frac{\sqrt{x^{2}+y^{2}} \times d x^{2} \times d \mathrm{X}}{2 y^{4}\left(d \frac{x}{y}\right)^{2}}$, in which, when $y$ and $d y$ are put in terms of $x$, as both numerator and denominator will be multiplied by $d x^{3}$, there will be no differential, and the force may be found in terms of the radical - that is, of $r$, though often complicated with $x$ also. It is generally advisable, having the equation of the curve, to find $p, r$, and $\mathrm{R}$, first by some of the above formulas, and then substitute those values, or $d p$ and $d r$, in either of the expressions for $\mathrm{F}, \frac{d p}{2 p^{3} d r}$ or $\frac{r}{2 p^{3} \mathrm{R}}$. 
To take an example in the parabola, where $\mathrm{S}$ being the focus, and $\mathrm{OS}=a, y^{2}=4 a x$, and $\mathrm{T} \mathrm{M}=2 x$, and $p=\mathrm{Y} \mathrm{S}=\sqrt{(a+x) a} ; r=\mathrm{S} \mathrm{P}=a+x$, and $\mathrm{R}=\frac{r d r}{d p}=2(a+x) \sqrt{\frac{a+x}{a}}$; we have therefore $\mathrm{F}$ as

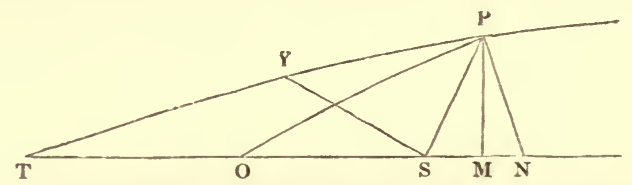

$\frac{r}{p^{3} \cdot \mathrm{R}}=$

$\frac{a+x}{(a(a+x))^{\frac{3}{2}} \times 2(a+x) \sqrt{\frac{a+x}{a}}}=\frac{a+x}{2 a(a+x)^{3}}=$

$\frac{1}{2 a(a+x)^{2}}=\frac{1}{2.0 \mathrm{~S} . \mathrm{SP}^{2}}$, or, because O S (the parameter) is constant, inversely as the square of the distance: And the other formula $\mathrm{F}$ as $\frac{d p}{p^{3} d r}$ gives the same result for the law of force, or $\frac{1}{4 \mathrm{SP}^{2}}$.

Again, in the ellipse, if $a$ be half the transverse axis, and $b$ half the conjugate, and $r$ the radius vector, we have $p=b \sqrt{\frac{r}{2 a-r}}$, and $d p=\frac{a b d r}{\sqrt{r}(2 a-r)^{\frac{3}{2}}} ;$ therefore the formula $\frac{d p}{p^{3} \cdot d} r$ becomes $\frac{a b d r}{b^{2} \sqrt{ }-\bar{r} \times r^{\frac{3}{2}} \times d r}=\frac{a}{b^{2} r^{2}}$, or the force is inversely as the square of the distance.

* This result coincides with the synthetical solution of Sir Isaac Newton in Prop. XIII. 
Lastly, as the equations are the same for the hyperbola, with only the difference of the signs, the value of the force is also inversely as $r^{2}$, or the square of the distance. In the circle $a=$ the radius $=r=p$; hence $\frac{r}{p^{3} \mathrm{R}}$ becomes $\frac{a}{a^{4}}$, which, being constant, the force is everywhere the same. But if the centre of forces is not that of the circle, but a point in the circumference, the force is as $\frac{1}{r^{5}}$.

Respecting centrifugal forces it may be enough to add, that if $v$ is the velocity and $r$ the radius, the centrifugal force $f$, in a circle, is as $\frac{v^{2}}{r}$. Also if $\mathrm{R}$ be the radius of curvature, $f$ for any curve is $=\frac{v^{2}}{\overline{\mathrm{R}}}$ When a body moves in a circle by a centripetal force directed to the centre, the centrifugal force is equal and opposite to the centripetal. Also the velocity in uniform motion, like that in a circle, being as $\frac{s}{t}$, the space divided by the time, and the arc being as the radius $r, f$ is as $\frac{s^{2}}{r \cdot t^{2}}$ or as $\frac{r}{t^{2}}$. If two bodies moving in different circles have the same centrifugal force, then the times are as $\sqrt{r}$. - It is to the justly celebrated Huygens that we owe the first investigation of centrifugal forces. The above propositions, except the second, are abridged from his treatise.*

- The rest of the investigation of centripetal forces is an expansion of the formulas above given, and their application to various cases, but chiefly to the conic sections. It may be divided into four branches. First, the rules

* Horologium Oscillatorium, ed. 1673, p. 159, App. 
are given for determining the central force required to make the body move in a given orbit of one of the four conic sections. Secondly, the inquiry becomes material how curves of a given kind, namely, the conic sections, may severally be found by merely ascertaining certain points in them, or certain lines which they touch, because this enables us to ascertain, among other things, the whole of a planet's orbit, from ascertaining certain points by actual observation. This branch of the subject is purely mathematical, consisting of the rules for drawing those curves through given points, or between, or touching given straight lines; and it is subdivided into two heads according as one or neither focus is given. The third object is to ascertain the motion, place, and times of bodies moving in given trajectories generally; and, among others, also of bodies descending, or retarded in ascending, by gravity. The fourth branch treats of the converse inquiry into the figures of the trajectories, and the places, times, and motion, when the nature of the centripetal force is known.

It is thus manifest that the great importance of motion in the Conic Sections made Sir Isaac Newton consider those curves in particular, before discussing the general subject of trajectories.

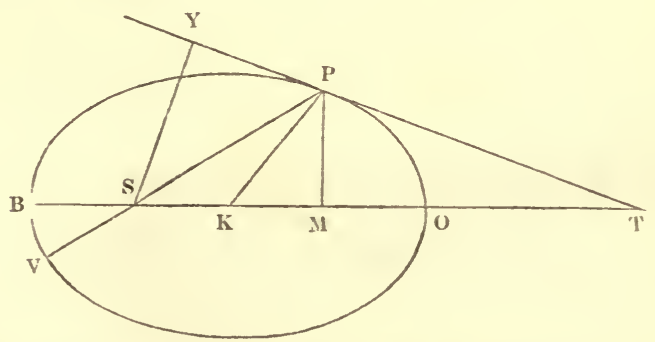

i. In exemplifying the use of the formulas we have E 2 
shown the proportion of the force to the distance in the conic sections generally, their foci being the centres of forces. Let us now see more in detail what the proportion is for the circle. Let $\mathrm{S}_{-}$be the centre of forces and $\mathrm{K}$ of the circle, $\mathrm{P}$ T a tangent, S Y a perpendicular to it, $\mathrm{K} \mathrm{M}$ and $\mathrm{MP}$ co-ordinates, $\mathrm{S} \mathrm{K}=b, \mathrm{KO}=a$, $\mathrm{P} \mathrm{M}=y$, and $\mathrm{M} \mathrm{K}=x$. Then, by similar triangles, $\mathrm{T} \mathrm{K} \mathrm{P}$ and $\mathrm{TSY}$, we have $\mathrm{SY}=\frac{\mathrm{ST} \times \mathrm{KP}}{\mathrm{TK}}$, or (because the sub-tangent $\mathrm{M} \mathrm{T}=\frac{y^{2}}{x}$, and $\left.a^{2}=x^{2}+y^{2}\right) \frac{a^{2}+b x}{a}$ or $\left(\frac{2 a^{2}+2 b x}{2 a}\right)$; also $\mathrm{S} \mathrm{P}=\sqrt{a^{2}+2 b x+b^{2}}$, and because by the property of the circle $\mathrm{OS} \times \mathrm{SB}$ or $(a+b)$ $(a-b)=a^{2}-b^{2}=\mathrm{P} \mathrm{S} \times \mathrm{S} \mathrm{V}$; therefore $\mathrm{S} \mathrm{V}=\frac{a^{2}-b^{2}}{\sqrt{a^{2}+2 b x+b^{2}}}$ and $\mathrm{P} \mathrm{V}=\frac{2 a^{2}+2 b x}{\sqrt{a^{2}+2 b x+b^{2}}}$.

Now by the formula already stated as Bernouilli's, but really Sir Isaac Newton's, the centripetal force in $\mathrm{P}$ is as $\frac{\mathrm{SP}}{\mathrm{SY}^{3} \times \mathrm{R}}, \mathrm{R}$ being the radius of curvature, and in the circle that is constant being $=a$, the semidiameter; therefore the force is as $\frac{\sqrt{a^{2}+2 b x+b^{2}}}{\frac{a\left(2 a^{2}+2 b x\right)^{3}}{8 a^{3}}}$, or as $\frac{8 \times a^{2} \sqrt{a^{2}+2 b x+b^{2}}}{\left(2 a^{2}+2 b x\right)^{3}}$, that is $\frac{\mathrm{B} \mathrm{O}^{2} \times \mathrm{S} \mathrm{P}}{\left(2 a^{2}+2 b x\right)^{3}}$; or as $\frac{\mathrm{B} \mathrm{O}^{2} \times \mathrm{S} \mathrm{P}^{3}}{\left(2 a^{2}+2 b x\right)^{3} \times \mathrm{S} \mathrm{P}^{2}}$, or as $\frac{\mathrm{BO}^{2}}{\frac{\left(2 a^{2}+2 b x\right)^{3}}{\mathrm{SP}^{3}} \times \mathrm{P} \mathrm{S}^{2}}$. But $\frac{2 a^{2}+2 b x}{\mathrm{SP}}$ $=\frac{2 a^{2}+2 b x}{\sqrt{a^{2}+2 b x+b^{2}}}=\mathrm{PV}$. Therefore the central 
force is as $\frac{\mathrm{BO}^{2}}{\mathrm{PV}^{3} \times \mathrm{SP}^{2}}$; or (because $\mathrm{OB}^{2}$ is constant) the central force is inversely as the square of the distance and the cube of the chord jointly. Of consequence, where $\mathrm{S}$ is in the centre of the circle and $b=o$, the force is constant, $\mathrm{SP}$ becoming the radius and $\mathrm{PV}$ the diameter; and if $\mathrm{S}$ is in the circumference of the circle as at $\mathrm{B}$, or $a=b$, then the chord and radius vector coinciding, the force is inversely as the fifth power of the distance, and is also inversely as the fifth power of the cosine of the angle P S O.

By a similar process it is shown that in an ellipse the force directed to the centre is as the distance. Indeed, a property of the ellipse renders this proof very easy. For if $\mathrm{SY}$ is the perpendicular to the tangent $\mathrm{T} \mathrm{P}$, and $\mathrm{N} \mathrm{P}$ (the normal) parallel to $\mathrm{SY}$, and $\mathrm{SA}$ the semi-conjugate

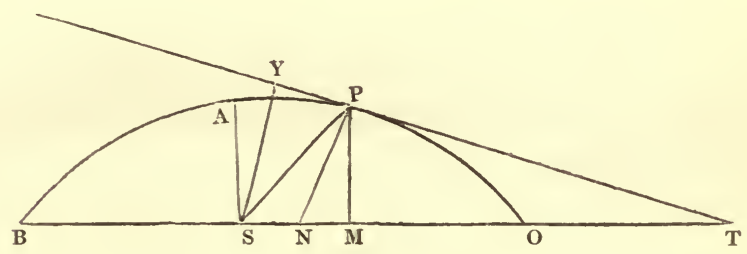

axis; $\mathrm{S} \mathrm{A}$ is a mean proportional between $\mathrm{SY}$ and $\mathrm{PN}$, and therefore $S Y=\frac{A S^{2}}{\mathrm{PN}^{-}}$; also the radius of curvature of the ellipse is (like that of all conic sections) equal to $\frac{4 \mathrm{P} \mathrm{N}^{3}}{\mathrm{P}^{2}}, \mathrm{P}$ being the parameter. Therefore we have to substitute these values for $\mathrm{S} \mathrm{Y}$ and the radius of curvature, $R$, in the expression for the central 
force, $\frac{\mathrm{SP}}{\mathrm{K} \times \mathrm{SY}^{3}}$, and we have $\frac{\mathrm{SP}}{\frac{4 \cdot \mathrm{PN}}{\mathrm{P}^{2}} \times \frac{\mathrm{A}^{6}}{\mathrm{PN}^{3}}}=$ $\frac{\mathrm{P}^{2}}{4 \mathrm{~A} \mathrm{~S}^{6}} \times \mathrm{SP}$; so that, neglecting the constant $\frac{\mathrm{P}^{2}}{4 \mathrm{AS}^{6}}$, the centripetal force is as the distance directly

From hence it follows, conversely, that if the centripetal force is as the distance, the orbit is elliptical or circular, for by reversing the steps of the last demonstration we arrive at an equation to the ellipse; or, in case of the two axes being equal, to the circle. It also follows that if bodies revolve in circular or elliptical orbits round the same centre, the centre of the figures being the centre of forces, and the force being as the distance, the periodic time of all the bodies will be the same, and the spaces through which they move, however differing in length from each other, will all be described in the same time. This proposition, which sometimes has appeared paradoxical to those who did not sufficiently reflect on the subject, is quite evident from considering that the corce and velocity being increased in proportion to the distance, and the lengths of similar curvilinear and concentric figures being in some proportion, and that always the same, to the radii, the lengths are to each other as those radii, and consequently the velocity of the whole movement is increased in the same proportion with the space moved through. Hence the times taken for performing the whole motion must be the same. Thus, if $\mathrm{V}$ and $v$ are the velocities, $\mathrm{R}$ and $r$ the radii, $\mathrm{S}$ and $s$ the lines described in the times $\mathrm{T}$ and $t$, by two such bodies round a common centre, $\mathrm{V}: v:: \mathrm{R}: r$, and $\mathrm{S}: s:: \mathrm{R}: r$; and because

$$
\mathrm{V}=\frac{\mathrm{S}}{\mathrm{T}} \text { and } v=\frac{s}{t}, \frac{\mathrm{S}}{\mathrm{T}}: \frac{s}{t}:: \mathrm{R}: r \text {, and } \mathrm{S}: s:: \mathrm{TR}:
$$


$t r$; or $\mathrm{R}: r:: \mathrm{TR}: t r ;$ and therefore $\mathrm{T}=t$. Hence if gravity were the same towards the sun that it is between the surface and centre of each planet, or if the sun were moved but a very little to one side, so as to be in the centre of the ellipse, the whole planets would revolve round him in the same time, and Saturn and Uranus would, like Mercury, complete their vast courses in about three of our lunar months instead of 30 and 80 years, - a velocity in the case of Uranus equal to 75,000 miles in a second, or nearly one-third that of light.

It also follows from this proposition that, if such a law of attraction prevailed, all bodies descending in a straight line to the centre would reach it in the same time from whatever distance they fell, because the elliptic orbit being indefinitely stretched out in length and narrowed till it became a straight line, bodies would move or vibrate in equal times. through that line. This is the law of gravity at all points within the earth's surface, and Sir I. Newton has alapted one of his investigations to it, when treating of the pendulum.

Another consequence of this proposition is, that if the centre of the ellipse be supposed to be removed to an infinite distance, and the figure to become a parabola, the centripetal force being directed to a point infinitely remote, becomes constant and equable; a proposition discovered first by Galileo.

Sir Isaac Newton having treated of the centripetal force in conic sections, where the centre of forces is the centre of the figure (and generally whatever be the centre in the case of the circle), proceeds to treat of that force where it is directed towards the focus of one or other of those curves, and not to the centre. It is easy to demonstrate a compendious theorem, that which forms the subject of his three first propositions, in which he determines the 
law of the force for the three curves (parabola, hyperbola, and ellipse) severally. For this purpose a simple reference to the formulas already stated will suffice; indeed our illustration of those formulas has already anticipated this.

If $\mathrm{OPA}$ be a conic section whose parameter is $\mathrm{D}$, S Y the perpendicular to the tangent T P, P R the radius of curvature at $\mathrm{P}$; then $\mathrm{SY}: \mathrm{SP}:: \frac{1}{2} \mathrm{D}$ : $\mathrm{PN}$ (the normal), and $\mathrm{SY}=\frac{\mathrm{D} \cdot \mathrm{S} \mathrm{P}}{2 \cdot \mathrm{PN}}$; also $\mathrm{PR}=$ $\frac{4 \mathrm{P} \mathrm{N}^{3}}{\mathrm{D}^{2}}$. Substitute these values of $\mathrm{S} \mathrm{Y}$ and $\mathrm{P} \mathrm{R}(p$ and $R$ ) in the expression formerly given for the central

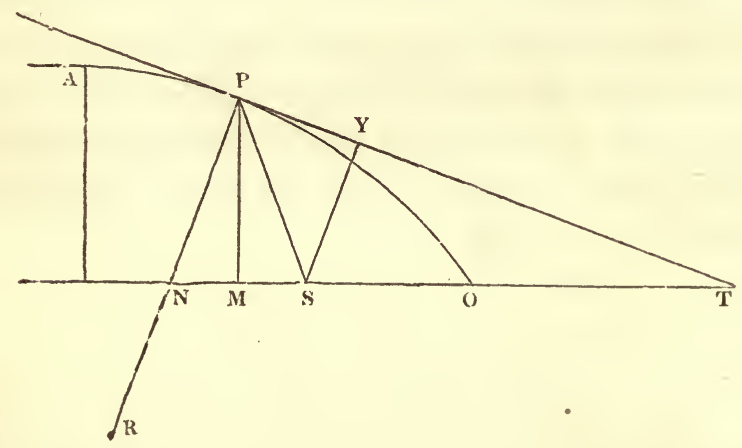

force $\frac{r}{p^{3} \times \mathrm{R}}$, and we have $\frac{\mathrm{S} \mathrm{P}}{\frac{\mathrm{D}^{3} \cdot \mathrm{SP}^{3}}{8 \mathrm{P} \mathrm{N}^{3}} \times \frac{4 \mathrm{P} \mathrm{N}^{3}}{\mathrm{D}^{2}} \text { or }}$ $\frac{2}{\mathrm{D} \times \mathrm{SP}^{2}}$, which is ( $\mathrm{D}$ being invariable) as the inverse square of the distance. Therefore any body moving in any of the conic sections by a force directed to the focus, is attracted oy a centripetal force inversely as the square of the distance from that focus. This demon- 
stration, therefore, is quite general in its application to all the conic sections.

It follows that if a body is impelled in a straight line with any velocity whatever, from an instantaneous force, and is at the same time constantly acted upon by a centripetal force which is inversely as the square of the distance from the centre, the path which the body describes will be one or other of the conic sections. For if we take the expression $\frac{1}{\mathrm{D} . \mathrm{S} \mathrm{P}^{2}}$ and work backwards, multiplying the numerator and denominator both by $\mathrm{S} P$, and then multiplying the denominator by $\frac{8 \mathrm{D}^{2} \cdot \mathrm{P} \mathrm{N}^{3}}{8 \mathrm{D}^{2} \cdot \mathrm{P} \mathrm{N}^{3}}$, we obtain the expressions for the value of $\mathrm{SY}$, the perpendicular, and for $R$, the radius of curvature. But no curves can have the same value of $S Y$ and $R$, except the conic sections; because there are no other curves of the second order, and those values give quadratic equations between the co-ordinates.

By pursuing another course of the same kind algebraically, we obtain an equation to the conic sections generally, according as certain constants in it bear one or other proportion to one another. The perpendicular $\mathrm{S} \mathrm{Y}$ and the radius of curvature are given in terms of the normal; and either one or the other will give the equation. Thus $\mathrm{R}=\frac{\left(d x^{2}+d y^{2}\right)^{\frac{3}{2}}}{d x^{2} \times d\left(\frac{d y}{d x}\right)}=\frac{4 \mathrm{P} \mathrm{N}^{3}}{\mathrm{D}^{2}}=\frac{4 y^{3}}{\mathrm{D}^{2} d x^{3}} \times\left(d x^{2}+d y^{2}\right)^{\frac{3}{2}}$ which gives $\mathrm{D}^{2} d x^{3}=4 y^{3} \times\left(d^{2} y d x-d^{2} x d y\right)$ an equation to the co-ordinates. Now whether this be resolvable or not, it proves that only one description of curres, of one order, can be such as to have the property in question. The former operation of going back from the expression of the central force, proves that the conic sec- 
tions answer this condition. Therefore no other curves can be the trajectories of bodies moving by a centripetal force inversely as the square of the distance.*

It may be remarked that J. Bernouilli objects (Mém. Acad. des Sciences, 1710) to Sir Isaac Newton that he had assumed the truth of this important proposition without any demonstration. But this is not correct. $\mathrm{He}$ certainly gives a very concise and compendious one; but he states distinctly that the focus and point of contact being given, and the tangent given in position, a conic section may be described which shall at that point of contact have a given curvature; that the curvature is given from the velocity and central force being given; and that two orbits touching each other with the same centripetal force and velocity cannot be described. This is in substance what we have expounded in the above demonstration. But it must also be observed, as Laplace has remarked, that Newton has in a subsequent problem shown how to find the curve in which a body must move with a given velocity, initial direction, and position; and since, when the centripetal force is inversely as the square of the distance, the curve is shown to be one or other of the conic sections, he has thus demonstrated the proposition in question; so that if he had not done so in the corollary to one problem, he has in the solution of another. $\uparrow$

J. Bernouilli objects also to a very concise and elegant

* The equation may be resolved and integrated ; there results, in the first instance, the equation $d x=\frac{2 y d y}{\sqrt{2 c y^{2}-\mathrm{D}^{2}}}$, and therefore the integral is this quadratic, $c^{2} x^{2}-2 c y^{2}-2 c \mathrm{C} x+\mathrm{C}^{2}+\mathrm{D}^{2}=0$. - Another demonstration is given in the Appendix, No. 2.

† Système du Monde, liv. v. ehap. 5. It is to be observed, that the Serenteenth Prop. Book I., is exactly the same in the first as in the subsequent editions, exeept the immaterial addition of a few lines to the demonstration. Consequently, Bernouilli must have been aware of it when he wrote in 1710 . 
solution of the inverse problem given by Herrman in the same volume of the Mémoires, and which had been communicated to him before it was presented to the Academy. This solution proceeds upon his general expression for the centripetal force, $-\frac{d^{2} x}{x} \sqrt{x^{2}+y^{2}}$; and the objection made is that he works the problem (as he does in a few lines) by multiplications and divisions which show that he was previously aware of the solution in the case of the conic sections. But this is no objection to a solution which being of a problem already known, can only be regarded as a demonstration that the former solution was exact. It is an objection which, if valid, applies certainly to the demonstration which we have just given of the proposition; but so it does to all the demonstrations of the ancient geometrical analysis. It is a more substantial objection that Herrman omitted a constant in his integration; but by adding it, Bernouilli shows that the equation which Herrman found, when thus corrected, expresses the conic sections generally.

This truth, therefore, of the necessary connexion between motion in a conic section and a centripetal force inversely as the square of the distance from the focus, is fully established by rigorous demonstration of various kinds.

If we now compare the motion of different bodies in concentric orbits of the same conic sections, we shall find that the areas which, in a given time, their radii vectores describe round the same focus, are to one another in the subduplicate ratio of the parameters of those curves. From this it follows, that in the ellipse whose conjugate axis is a mean proportional between its transverse axis and parameter, the whole time taken to revolve (or the periodic time) being in the proportion of the area (that is in the proportion of the rectangle of the axes) directly, and in 
the subduplicate ratio of the parameter inversely, is in the sesquiplicate ratio of the transverse axis, and equal to the periodic time in a circle whose diameter is that axis. It is also easy to show from the formula already given respecting the perpendicular to the tangent, that the velocities of bodies moving in similar conic sections round the same focus, are in the compound ratio of the perpendiculars inversely and the square roots of the parameters* directly. Hence in the parabola a very simple expression obtains for the velocity. For the square of the perpendicular being as the distance from the focus by the nature of the curve (the former being $a^{2}+a x$, and the latter $a+x$ ), the velocity is inversely as the square root of that distance. In the ellipse and hyperbola where the square of the perpendicular varies differently in proportion to the distance, the law of the velocity varies differently also. The square of the perpendicular in the ellipse (A being the transverse axis and $\mathrm{B}$ the conjugate, and $r$ the radius vector) is $\frac{\mathrm{B}^{2} \times r}{\mathrm{~A}-r}$; in the hyperbola, $\frac{\mathrm{B}^{2} \times r}{\mathrm{~A}+r}$, or those squares of the perpendicular vary as $\frac{r}{\mathrm{~A}-r}$ and $\frac{r}{\mathrm{~A}+r}$, in those curves respectively, $\mathrm{B}^{2}$ being constant. Hence the velocities of bodies moving in the former curve vary in a greater ratio than that of the inverse subduplicate of the distance, or $\frac{1}{\sqrt{r}}$, and in a smaller ratio in the latter curve, while in the parabola $\frac{1}{\sqrt{r}}$ is their exact measure.

To these useful propositions, Demoivre added a theorem of great beauty and simplicity respecting motion in the

* By parameter is always to be understood, unless otherwise mentioned, the principal parameter, or the parameter to the principal diameter. 
ellipse. The velocity in any point $\mathrm{P}$ is to the velocity in $\mathrm{T}$, the point where the conjugate axis cuts the curve, as the square root of the line joining the former point $\mathrm{P}$ and the more distant focus, is to the square root of the line joining $\mathrm{P}$ and the nearer focus. It follows from these propositions that in the ellipse, the conjugate axis being a mean proportional between the transverse and the parameter, and the periodic time being as the area, that is as the rectangle of the axes directly, and the square root of the parameter inversely, $t$ being that time, $a$ and $b$ the axes, and $p$ the parameter, $t=\frac{a b}{\sqrt{p}}$, and $b^{2}=a p$; therefore $a b=a \sqrt{a p}=\sqrt{ } a^{3} \times \sqrt{ } \bar{p}$; and $t=$ $\sqrt{a^{3}}$, and $t^{2}=a^{3}$; or the squares of the periodic times are as the cubes of the mean distances. So that all Kepler's three laws have now been demonstrated, à priori, as mathematical truths; first, the areas proportional to the times if the force is centripetal-second, the elliptical orbit, -and third, the sesquiplicate ratio of the times and distances, if the force is inversely as the squares of the distances, or in other words if the force is gravity.

Again, if we have the velocity in a given point, the law of the centripetal force, the absolute quantity of that force in the point, and the direction of the projectile or centrifugal force, we can find the orbit. The velocity in the conic section being to that in a circle at the given distance $\mathrm{D}$ as $m$ to $n$, and the perpendicular to the tangent being $p$, the lesser axis will be $\frac{2 m p}{\sqrt{2 n^{2}-m^{2}}}$, and the greater axis $\frac{2 \mathrm{D} n^{2}}{2 n^{2}-m^{2}}$, the signs being reversed in the denominator of each quantity for the case of the hyperbola. Hence the very important conclusion that the length of 
the greater axis does not depend at all upon the direction of the tangential or projectile force, but only upon its quantity, the direction influencing the length of the lesser axis alone.

Lastly, it may be observed, that as these latter propositions give a measure of the velocity in terms of the radius vector and perpendicular to the tangent for each of the conic sections, we are enabled by knowing that velocity in any given case where the centripetal force is inversely as the square of the distance, and the absolute amount of that force is given, as well as the direction of the projectile force and the point of the projection, to determine the parameters and foci of the curve, and also which of the conic sections is the one described with that force. For it will be a parabola, an hyperbola, or an ellipse, according as the expression obtained for $p^{2}$ (the square of the perpendicular to the tangent) is as the radius vector, or in a greater proportion, or in a less proportion. This is the problem above referred to, which John Bernouilli had entirely overlooked, when he charged Sir Isaac Newton with having left unproved the important theorem respecting motion in a conic section, which is clearly involved in its solution.

Before leaving this proposition, it is right to observe that the two last of its corollaries give one of those sagacious anticipations of future discovery which it is in vain to look for anywhere but in the writings of this great man.* He says, that by pursuing the methods indicated in the investigation, we may determine the variations impressed upon curvilinear motion by the action of disturbing, or, what he terms, foreign forces; for the changes intro-

* See a singular anticipation respecting dynamics, by Lord Bacon, in $D e$ Ang. Lib. III., under the head Translation of Experiments. It was pointed out to me by my learned friend B. Montague. 
duced by these in some places, he says, may be found, and those in the intermediate places supplied, by the analogy of the series. This was reserved for Lagrange and Laplace, whose immortal labours have reduced the theory of disturbed motion to almost as great certainty as that of untroubled motion round a point by virtue of forces directed thither.*

We have thus seen how important in determining all the questions, both direct and inverse, relating to the centripetal force, are the perpendicular to the tangent and the radius of curvature. Indeed it must evidently be so, when we consider, first, that the curvature of any orbit depends upon the action of the central force, and that the circle coinciding with the curve at each point, beside being of well-known properties, is the curve in which at all its points the central force must be the same; and, secondly, that the perpendicular to the tangent forms one side of a triangle similar to the triangle of which the differential of the radius vector is a side; the other side of the former triangle being the radius vector, the proportion of which to the force itself is the material point in all such inquiries. The difficulty of solving all these problems arises from the difficulty of obtaining simple expressions for those two lines, the perpendicular $p$ and the radius of curvature $R$. The radius vector $r$ being always $\sqrt{x^{2}+y^{2}}$ interposes little embarrassment; but the other two lines can seldom be concisely and simply expressed. In some cases the value of $\mathrm{F}$, the force, by $d r$ and $d p$ may be more convenient than in others; because $p$ may involve the investigation in less difficulty than $\mathrm{R}$; besides that $p^{3}$ enters into the expression which has no differentials. But in the greater number of

* Laplace (Méc. Cél. lib. xv. ch. i.) refers to this remarkable passage as the germ of Lagrange's investigations in the Berlin Mémoires for 1786 . 
instances, especially where the curve is given, the formula $\frac{r}{p^{3} R}$ will be found most easily dealt with.

ii. The next branch of the inquiry relates to the describing the conic sections severally, where certain points are given through which they are to pass, or certain lines which they are to touch. The subject is handled in two sections, (the fourth and fifth,) the first of which treats the case where one of the foci is given; the second the case where neither focus is given. This whole subject is purely geometrical; and exhibits a fertility of resources in treating these difficult problems, as well as an elegance in the manner of their solution, which has few parallels in the history of ancient or modern geometry. This portion of the Principia, however, is incapable of abridgment; and there is no advantage whatever in resolving the problems analytically, but rather the contrary; for with the exception of one of the lemmas, in demonstrating which Sir Isaac Newton himself has recourse to algebraical reasoning in order to shorten the proofs, the geometrical process is in almost every instance extremely concise, in all cases much more beautiful, and less encumbered than the algebraical. The superiority of the former to the latter method of investigation in such solutions is apparent on trying algebraically some simple case, as that of describing a circle through three points, or through two points and touching a line given in position; no little embarrassment results from the number and entanglement of the quantities in the solution. Even so great a master of analysis as Sir Isaac Newton, in solving the problem of describing a circle through two points, and touching a given line, could find no better expression than $x=\frac{-e^{2} b \sqrt{e^{2} b^{2}+e^{2} a^{2}-d^{2} a}}{d^{2}-a^{2}}$, although geometrically the construction is easy by drawing a circle 
on one segment of the line joining the given points, and another on the given line.* These are comparatively simple problems; in the more difficult cases of the conic sections this embarrassment is often inextricable. $\dagger$

To illustrate the application of these important problems, let us suppose that by observation we obtain three points in the orbit of any planet, and would ascertain from those points the position of the greater axis, and the focus in which the sun is placed, the eccentricity of the orbit or distance of the focus from the centre of the ellipse, and the aphelion, or greatest distance to which in its course the planet ever is removed from the sun; this is easily done by means of Prop. XVIII. (Book I.), for that enables us to find the elliptical and hyperbolical trajectories, which pass through given points, when one focus and the transverse axis are given; and thus to find the other focus, and the centre of the curve, and the distance from the given focus to the further extremity of the axis, which is the aphelion.

In like manner the problem which Sir Isaac Newton calls by far the most difficult of any, and says that he had tried to solve in various wayst, that of finding the trajectory of a comet from three observations, supposing it to move in a parabolic orbit, is reduced by an elaborate and difficult process of reasoning to describing a parabola through two given points, which are found in its own orbit from the observations. Now Prop. XIX. of Book I. gives an easy solution of this problem. $\S$ It is only to

* The abore algebraical solution is that of Prop. 43. of the Arith. Univ., where Props, 59, 60, and 61. are also solutions of the three first problems of Sect. V. of the Principia, B. I.

† Maria Agnesi's Instituzioni Analitiche abounds in elegant algebraical investigations of geometrical problems, but affords no grounds for modifying the above remark.

‡ Problema hocce longe difficillimum multimode aggressus (Lib. III. Prop. 41.).

$\S$ Several other propositions are given in the first book for the purpose 
describe from each of the given points a circle, with the distance of that point from the given focus as a radius, and the straight line touching these two circles will be the directrix of the parabola, and the perpendicular to it from the focus, its axis; the principal vertex being the middle point of that perpendicular. The coincidence of the very eccentric elliptical orbits of the comets with the parabola, makes this parabolic hypothesis answer for determining their places and times in the general case.

The correction of the orbit thus found is reduced to finding the orbit of an ellipse which shall pass through three given points, and this is done by the 21 st proposition of Book I., or rather by the 16 th lemma, to which it is a corollary, for inflecting three straight lines from three given points, the differences, if any, between the lines, being given.

Sir Isaac Newton tried the accuracy of the methods thus found upon several comets, and particularly on the celebrated one of 1680 , called Halley's comet, from the great labour which that mathematician, in aid of his illustrious friend and master, bestowed upon the calculation of its orbit. The following is a short statement of the general result of a comparison between the places computed from the theory, and the places found by actual observation, in the cases tried.

of facilitating the solution of this difficult problem by another method; but the author informs us that he subsequently fell upon the method which he has given in the third book, and which he prefers for its greater simplicity. - It is, however, very remarkable that he overlooked the important circumstance of there being a porism connected with his solution, or a case in which the problem becomes indeterminate and has an infinite number of solutions; and what is still more singular that the case of the comet is that of the porism, - so that the solution is wholly inapplicable. This was first discovered by F. Boscovich in 1749 ; it being found that the solution had thrown the comet upon the wrong side of the sun. (See Life of Simson, Appx.) 
First, as regards the comet of 1680 , or Halley's comet.

In comparing four observations with the geometrical computation, Sir Isaac Newton found an error of $5^{\prime} 3^{\prime \prime}$ on an average in the latitude, and about $1^{\prime}$ in the longitude. But Halley, having afterwards made the computations with greater accuracy by arithmetical operations, found the average error, on sixteen observations, in the latitude only about $52^{\prime \prime}$, and in the longitude $1^{\prime} 28^{\prime \prime}$. The average error found on a comparison of the theory with twentyone observations made abroad, was found by Halley only to be $50^{\prime \prime}$ in the latitude, and $57^{\prime \prime}$ in the longitude.*

Secondly, as regards other comets.

In the computations of the comet 1665 , the error was, on an average of eighteen observations, $8^{\prime \prime}$ in the latitude, and in the longitude $1^{\prime} 25^{\prime \prime}$. In the latitude the errors by excess nearly balance those by defect, the one being to the other as 40 to 49 . In the longitude, supposing the observation of December 7 accurately stated (which, from the error amounting to $7^{\prime} 33^{\prime \prime}$, seems very doubtful), the errors by excess are sixteen times more considerable than those by defect. In the comets of 1682 and 1683, on comparing the observations of Flamstead with the theory, the error was $1^{\prime} 31^{\prime \prime}$ in latitude, and $45^{\prime \prime}$ in longitude, for eleven observations of the former comet, and for seventeen of the latter comet, $1^{\prime} 10^{\prime \prime}$ in latitude, and $1^{\prime} 29^{\prime \prime}$ in longitude. But the comet of 1723 came nearer its computed place; the average error of latitude on fifteen observations of Bradley, compared with the same number by Halley himself, and Pound (his uncle), was only $21^{\prime \prime} \frac{1}{2}$ in the latitude, and somewhat under $25^{\prime \prime}$ in the longitude. It is to be remarked that this is apparently the case in which the

* This omits the observation made 26th December, as there is manifestly an error in the figures of that observation. 
observations were the most accurate, three eminent observers checking each other, and no one observation differing from the computation much more than by the average of the rest, while great differences occur in all the other cases, and give rise to a suspicion of error. For in the comet of 1683, there was one day (Aug. 15) in which the latitude differed between three and four times, and the longitude three times more than the average; and in the observations of the comet of 1665 there are several errors in longitude of twice, and one error of no less than five times, above the average. These particular observations, and not the theory, then, were probably at fault in those instances; but they affect the general average materially.

The intimate connection between the purely geometrical parts of the Principia, the Fifth and Sixth Sections of the First Book, and the most sublime inquiries into the motions of the heavenly bodies, those motions, too, which are the most rapid, and performed in spaces the most prodigious, may suffice to show the student how well worthy these mathematical investigations are of being minutely followed. Were they wholly unconnected with such important speculations in Physical Astronomy, and only to be regarded as a branch of the Higher Geometry, they would deserve the deepest attention, for their interesting development of general relations between figures so well known as the conic sections, for the marvellous felicity of the expedients by which the solutions are obtained, and for the inimitable elegance with which the reasoning is conducted. As a mere matter of mathematical contemplation, beginning and ending in the discovery of the relations which subsist between different quantities and figures, they afford matter of lasting interest to the geometrician. But it certainly heightens that interest to reflect that the same skilful and simple construction which enables us to describe a para- 
bola through given points, or touching given lines, beside gratifying a curiosity purely geometrical, leads us to calculate within $20^{\prime \prime}$ of the truth the place of bodies revolving round the sun in orbits so eccentric that the ellipse which they describe coincides with a parabolic line, instead of being nearly circular like the path of our globe, although our own distance from that luminary is near a hundred millions of miles.

iii. We are next /to consider the motion of bodies in conic sections which are given, and (ascending or descending in straight lines under the influence of gravity; that is, the velocities and the times of their reaching given points, or their places at given times. This branch of the subject, therefore, divides itself into two parts, the one relating to motion in the conic sections, the other to the motion of bodies ascending or descending under the influence of gravitation. The Sixth Section treats of the former, the Seventh of the latter.

(1.) In order to find the place of a revolving body in its trajectory at any given time, we have to find a point such that the area cut off by the radius vector to that point shall be of a given amount; for that area is proportional to the time. Thus, suppose the body moves in a parabola, and that its radius vector completes in any time a certain space, say in half a year moves through a space making an area equal to the square of $\mathrm{D}$; in order to ascertain its position in any given day of that half year, we have to cut off, by a line drawn from the centre of forces, an area which shall bear to $\mathrm{D}^{2}$ the same proportion that the given time bears to the half year, say 3 to $\mathrm{m}^{2}$, or we

have to cut off a section A S P $=\frac{3}{m^{2}} \mathrm{D}^{2}$, A P being the parabola and $\mathrm{S}$ the focus. This will be done if $\mathrm{AB}$ 
be taken equal to three times $\mathrm{A} \mathrm{S}$, and $\mathrm{BO}$ being drawn perpendicular to $\mathrm{A} \mathrm{B}$, between $\mathrm{B} \mathrm{O}, \mathrm{B} \mathrm{A}$ asymptotes, a

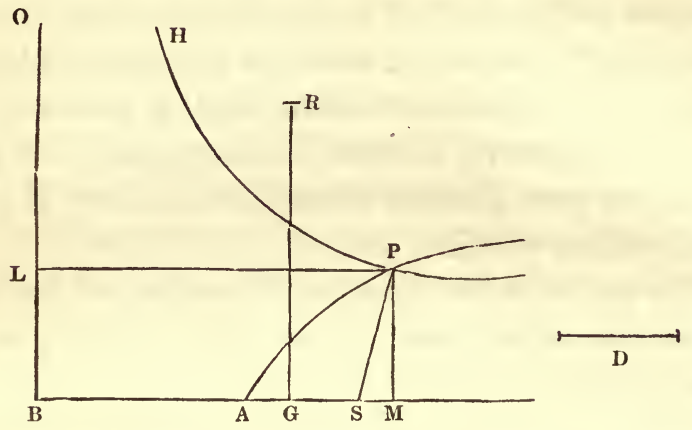

rectangular hyperbola is drawn, $\mathrm{HP}$, whose semi-axis or semi-parameter is to $\mathrm{D}$ in the proportion of 6 to $\mathrm{m}$; it will cut the parabolic trajectory in the point $\mathrm{P}$, required. For calling $\mathrm{A} \mathrm{M}=x$ and $\mathrm{P} \mathrm{M}=y$ and $\mathrm{A} \mathrm{S}$ $=a$; then $\mathrm{AB}=3 a$ and $y \times(x+3 a)=$ half the square of the hyperbola's semi-axis, which axis being equal to $\frac{6 \mathrm{D}}{m}, y(x+3 a)=\frac{36 \mathrm{D}^{2}}{2 m^{2}}=\frac{18 \mathrm{D}^{2}}{m^{2}}$, or $y\left(\frac{x}{3}+a\right)$ $=\frac{6 \mathrm{D}^{2}}{m^{2}}$, and $y\left(\frac{x}{6}+\frac{a}{2}\right)=\frac{3 \mathrm{D}^{2}}{m^{2}}$, or $y \times\left(\frac{2}{3} x-\frac{1}{2} x+\frac{1}{2} a\right)$ $=\frac{3 \mathrm{D}^{2}}{m^{2}}$. Therefore $\frac{2}{3} x y-\frac{1}{2}(x-a) y=\frac{3 \mathrm{D}^{2}}{m^{2}}$, and $\frac{2}{3}, \mathrm{~A} \mathrm{M} \times \mathrm{P} \mathrm{M}=\frac{2}{3} x y ;$ and $\frac{1}{2}(x-a) y=\frac{1}{2}$ $\mathrm{S} \mathrm{M} . \mathrm{P} \mathrm{M}=\mathrm{S} \mathrm{M} \mathrm{P}$; therefore the sector A S P $=\frac{3 \mathrm{D}^{2}}{m^{2}}$ : so that the radius from the focus $\mathrm{S}$ cuts off the given area, and therefore $\mathrm{P}$ is the point where the comet or other body will be found in $\frac{3}{m^{2}}$ parts of the time.

If the point is to be found by computation, we can easily find the value of $y$ by a cubic equation, $y^{3}+3 a^{2}$ 
$y=\frac{18 a^{2} \mathrm{D}^{2}}{m^{2}}$, and making $\mathrm{BL}=y, \mathrm{~L} \mathrm{P}$ parallel to A M, cuts $\mathrm{A} \mathrm{P}$ in the point $\mathrm{P}$ required. Sir Isaac Newton gives a very elegant solution geometrically by bisecting $\mathrm{A} \mathrm{S}$ in $\mathrm{G}$, and taking the perpendicular $\mathrm{G} \mathrm{R}$ to the given area as 3 to $4 \mathrm{~A} \mathrm{~S}$, or to $\mathrm{SB}$, and then describing a circle with the radius $\mathrm{R} S$; it cuts the parabola in $\mathrm{P}$, the point required.* This solution is infinitely preferable to ours by the hyperbola, except that the demonstration is not so easy, and the algebraical demonstration far from simple.

It is further to be observed, that the place being given, either of these solutions enables us to find the time. Thus, in the cubic equation, we have only to find $\frac{3 \mathrm{D}^{2}}{m^{2}}$. It is equal to $\frac{y^{3}+3 a^{2} y}{6 a^{2}}$; and as $\mathrm{D}^{2}$ is the given integer, or period of $e . g$. half a year, the body comes to the point $\mathrm{P}$ in a time which bears to $\mathrm{D}^{2}$ the proportion of unity to $\frac{6 a^{2} \mathrm{D}^{2}}{y^{3}+3 a^{2} y}$.

Sir Isaac Nerrton proceeds to the solution of the same important problem in the case of the ellipse, which is that of the planetary system, and is termed Kepler's problem from having been proposed by him when he had discovered by observation that the planetary motions were performed in this curve, and that the areas described by the radii were proportional to the times. In the parabola which is quadrable and easily so, the area being twothirds of the rectangle under the co-ordinates, the solution of this problem is extremely easy. But the ellipse not

* The most singular relation subsists between the hyperbolas and parabolic areas, giving rise to very curious Porisms connected with Quadratures.-See Phil. Trans. 1798, part ii. 
admitting of an expression for its area, or the area of its sectors, in finite terms of any product of straight lines, the problem becomes incapable of a definite solution. Newton accordingly begins his investigation by a lemma, in which he endeavours to demonstrate that no figure of an oval form, no curve returning into itself and without touching any infinite arch, is capable of definite quadrature. It is rarely, indeed, that the expression "endeavour," can be applied to Sir Isaac Newton. But some have questioned the conclusiveness of his reasoning in this instance. The demonstration consists in supposing a straight line to revolve round a point within the oval, while another point moves along it with a velocity as the square of the portion of the revolving line between the given centre and the oval, that is, as the radius vector of the oval from the given centre. It is certainly shown, that the moving point describes a spiral of infinite revolutions; and, also, that its radius is always as the area of the oval at the point where that radius meets the oval. If then the relation between the area and any two ordinates from the oval to any axis is such as can be expressed by a finite equation, so can the relation between the radius of the spiral and co-ordinates drawn parallel to the former, or the coordinates to the same axis. Therefore it will follow, that the spiral can be cut only in a finite number of points by a straight line, contrary to the nature of that curve. Indeed, its co-ordinates being related to each other by an algebraical equation is equally contrary to its nature; consequently the possibility of expressing the relation between the area of the oval and the co-ordinates leads to this absurd conclusion, and therefore that possibility cannot exist; and hence it is inferred that the oval is not quadrable.

Sir Isaac Newton himself observes that this demonstration does not apply to ovals which form parts of curves, 
being touched by branches of infinite extent. But it does not even apply to all cases of ovals returning into themselves, and unconnected with any infinite branches. There is, for example, a large class of curves of many orders, those whose equation is $y^{m}=n^{m} x^{(n-1) m} \times\left(a^{n}-x^{n}\right)$; and when $m$ is even these curves are quadrable; and in every case where $m$ and $n$ are whole positive even numbers, it is the equation to a curve returning into itself. This is manifest upon inspection: for $\int y d x=\int n x^{n-1}$ $\left(a^{n}-x^{n}\right)^{\frac{1}{m}} d x$ is integrable because the power of $x$ without is one less than that of $x$ within the radical sign; and because there is no divisor there can be no asymptote; while it is plain that the $\frac{1}{m}$ root of $a^{n}-x^{n}$ is impossible when either $+x$ or $-x$ is greater than $a, n$ and $m$ being both whole even numbers. Wherefore the curve returns into itself; and as $y=0$, both when $x=0$, and when $x=+a$, or $-a$, therefore the figure consists of two ovals meeting or touching in the origin of the abscissæ. These two ovals admit of a perfect quadrature; the integral being $\mathbf{C}-\frac{m}{n(m+1)}\left(a^{n}-x^{n}\right)^{\frac{m+1}{m}}$. Thus if $m=n=2$ the area is $\mathrm{C}-\frac{1}{3}\left(a^{2}-x^{2}\right) \frac{3}{2}$, the latter quantity being one-half of an area that has to one-third the rectangle of the co-ordinates the same proportion which the difference of the squares of the diameter and abscissa has to the square of the abscissa; for $\frac{2}{3}\left(a^{2}-x^{2}\right)^{\frac{3}{2}}=\frac{1}{3} x y \times$ $\frac{a^{2}-x^{2}}{x^{2}}$.

The particular inquiry respecting motion in the ellipse did not perhaps require the proposition to be proved in the very general form in which Sir Isaac Newton has 
given it. That the ellipse cannot be squared might perhaps be sufficiently proved from this consideration, founded upon a reasoning analogous to that on which the lemma in question proceeds. - If a curve be described such that its co-ordinates, or the rectangle contained by the co-ordinates, shall always bear a given proportion to the areas of the ellipse on the same axis, this curve cannot be algebraical, not merely because of its equation involving quantities not integrable (for that may be said to be the question), but because it will stop short at a given line, which no algebraical curve can do. It will have no branch extending beyond the perpendicular at the end of the axis : and moreover its equation is known to be that of a transcendental curve. This reason cannot be applied to all curves returning into themselves; because, as we have seen in one class, the equation to the curve, whose co-ordinates should express their areas, is algebraical; and also because, in that class, the secondary curve is found to have two branches which meet in cusps, and so do not stop short. If described by the proportion of areas they would seem to stop short, that property only belonging to one of their branches; but their equation discloses the second branch. It is one of many instances of a truth perhaps not sufficiently remarked by geometricians, that curves sometimes have particular portions to which certain properties belong exclusively, no other part of the curve having them.

As the area of the ellipse cannot be found by algebraical quantities, or by the description of algebraical curves, the problem of Kepler cannot be solved otherwise than by transcendental curves, logarithms, circular arcs, or approximation. Sir Isaac Newton gives a solution by means of the cycloid described on an axis at right angles to the transverse axis of the ellipse, at a distance from its vertex which is a fourth proportional to half the trans- 
verse axis, the focal distance, and the eccentricity, and with a generating circle whose radius is the distance of this perpendicular from the centre. A parallel to the cycloid's axis, at the point whose abscissa is to the periphery of the generating circle in the proportion of the given time to the periodic time, cuts the ellipse at the place required. This solution requires a construction beside that of the curve described; but a cycloid may be described which shall cut the ellipse directly at the point required. If a circle is described on A B the transverse axis, and its quadrant $A k$ is cut in $\mathrm{O}$, in the given ratio of the times in which the elliptical area is to be cut; and then a cycloid is described, whose ordinate $\mathrm{P} M$ is always a fourth proportional to the arch $\mathrm{OQ}$, the rectangle of the two axes and the distance between the foci; or to $\mathrm{OQ}, \mathrm{A} \mathrm{B} \times$ 2. C E, and 2. C S, - this cycloid cuts the ellipse in the point required, $\mathrm{P}$. The equation to this curve $\mathrm{G} P$ is simple

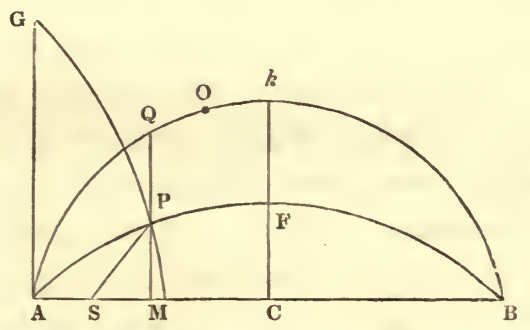

enough, and the construction easy; for the ordinate is in a given proportion to the $\operatorname{arc} \mathrm{Q} \mathrm{O}$ of the quadrant. As, however, an arithmetical approximation by means of series is required in practice, Sir Isaac Newton gives two methods, both of great elegance and efficiency.

It may be proper here to note the names given by astronomers to the lines and angles in the ellipse connected mainly with the investigation of this problem. The sun being in the focus $\mathrm{S}$, and $\mathrm{P}$ the planet's place, the aphelion 
of the planet is $\mathrm{B}$; the perihelion $\mathrm{A}$; the $\operatorname{arch} \mathrm{BP}$, or angle $\mathrm{BSP}$ is the true anomaly; $\mathrm{BO}$ being to the whole circumference as the time in $\mathrm{BP}$ to the whole periodic time, $\mathrm{BO}$, or $\mathrm{OSB}$, is the mean anomaly, and $\mathrm{QB}$, or $\mathrm{QCB}$, is the eccentric anomaly, $\mathrm{C}$ being the centre of the ellipse: A and B are likewise called the apsides (or apses),

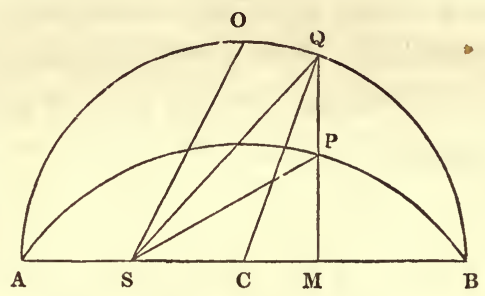

and $A B$, the transverse axis, is called the line of the apsides; S C, or more generally $\frac{\mathrm{S} \mathrm{C}}{\mathrm{AC}}$ is the eccentricity.

(2.) The next subject of inquiry is the comparison of bodies moving in a straight line towards the centre of forces, with those moving by the same centripetal force in the conic sections whose axis is that straight line. If the projectile force by which a body revolves in any of those curves round the focus as a centre, suddenly ceases, and the body falls towards the centre of the curve, it is shown that its place at any given time, will be the point at which the line of descent is cut by a perpendicular from the point of the curve where the radius from the vertex makes its area proportional to the time consumed in the fall. For take the parabola whose area is $\frac{2}{3} x y$, and let the distance of the point where the body begins to descend in a straight line be $\mathrm{C}$; the parabolic sectors, which are as the times, are expressed by $y \times\left(\frac{x+3 \mathrm{C}}{6}\right)\left(=\frac{2}{5} x y+\right.$ $\left.(\mathrm{C}-x) \frac{y}{2}\right)$ or $\frac{\sqrt{a x}}{6} \times(x+3 \mathrm{C})$; and if another parabola 
with the same vertex, and with a smaller parameter, $b$, is drawn nearer the straight line, its sectors are $\frac{\sqrt{b x}}{6}(x+3 \mathrm{C})$. Now the times in the first parabola, or the areas, at any two points referred to the abscissa $x$ and $z$, being $\frac{\sqrt{a x}}{6}(x+3 \mathrm{C})$, and $\frac{\sqrt{a z}}{6}(z+3 \mathrm{C})$, the times or areas in the second parabola will be $\frac{\sqrt{b x}}{6}(x+3 \mathrm{C})$, and $\frac{\sqrt{b z}}{6}$ $(z+3 \mathrm{C})$, respectively; and therefore it is evident that the areas at the distances $x$ and $z$, in the one curve are in the same proportion to one another with the areas in the other curve at those distances. If the parameter be continually diminished of the second curve, until that curve coincides with the axis, the same proportion holds; and the times, therefore, in falling through the axis, will be as the areas of the first curve, corresponding to the points of that axis. - And so it may be shown in the ellipse and hyperbola.

Hence it follows, that in the case of the parabola, the velocity of the falling body in any given point is equal to that with which the body would, moving uniformly, describe a circle having for its centre, the centre to which the body is falling, and for its diameter the distance of the given point from that centre. In the circle, the velocity at the given point is to the velocity in the circle described from the centre, with the distance of the given point for the radius, as the square root of the distance fallen through to that of the whole distance of the point where the fall begins. Thus let $d$ be the distance of the given point to which the body has fallen, $\mathrm{D}$ the distance of the point at which it began to fall; the velocity in the case of a parabola is equal to that of the body moving in a circle, whose 
radius is $\frac{1}{2} d$; in the case of a circle, it is to that of a body moving in a circle whose radius is $d$, as $\sqrt{\mathrm{D}-d}$ : $\sqrt{\mathrm{D}}$. - And the like proportion subsists in the case of the hyperbola.

Further, a rule is thus deduced for determining, conversely, the time of descent, the place being given. A circle is to be described on $\mathrm{A} \mathrm{S}=\mathrm{D}$, as the diameter, and another from $\mathrm{S}$ the centre, towards which the body falls, with the radius $\frac{\mathrm{D}}{2}$. $\mathrm{P}$ being the point to which it has fallen, if the area $\mathrm{S} \times \mathrm{X} \mathrm{B}$ be taken equal to SC A, the time taken to fall through $\mathrm{AP}$ is equal to the time

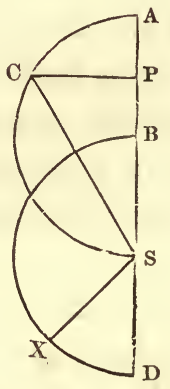

in which the body would move uniformly from $\mathrm{B}$ to $\mathrm{X}$. Hence the periodic times being in the sesquiplicate ratio of the distances $\left(t=d^{\frac{3}{2}}\right)$ and because $2^{\frac{3}{2}}=2 \sqrt{2}$, the time taken to fall through the whole distance to the centre is to the periodic time of a body revolving at twice that distance round the same centre as 1 to $4 \sqrt{2}$; and thus we can calculate the time (supposing the planetary orbits to be circular) which any one would take to fall in a straight line to the sun, or any satellite to its principal planet, if the projectile motion were suddenly to cease. 
The moon in this way would fall to the earth in about four hours less than five days.*

The inquiry is closed with a solution of the general problem, of which the preceding solutions for the conic sections, and for the force inversely as the squares of the distances, are only particular cases; and the times and velocities are found from the places, or the places from the times and velocities, where a body ascends from or descends to the centre, influenced by a centripetal force of whatever kind. On the given straight line of ascent or descent a curve is to be described whose co-ordinates are the centripetal force at each point of the axis, or whose equation is $y=\mathrm{X}, \mathbf{X}$ being a function of $x$, the distance from the beginning of the motion. The area of the curve at each point is $\int y d x=\int \mathbf{X} d x$; and if that integral is equal to $\mathrm{Z}^{2}, \mathrm{Z}$ is as the velocity at the distance $a-x$, from the centre. Another curve described on the same axis, and whose equation is $u=\frac{1}{\mathrm{Z}}$, gives by its areas $\int \frac{d x}{\mathrm{Z}}$ $=\zeta$, the time taken to move through the distance $a-x$; it is equal to 3 . This is easily demonstrated; for, first, if the velocity be $v$, and the time $d t$, the space being $d x$, we have the force $y=\frac{d v}{d t}$; and as $d t=\frac{d x}{v}$, therefore $y=\frac{v d v}{d x}$, and $y d x=v d v$, and $\int y d x=\frac{v^{2}}{2}$; but $\mathrm{Z}^{2}=\int y d x$; therefore $\mathrm{Z}=\frac{v}{\sqrt{2}}$, and the velocity is as the area Z. - Again; for the time in the other curve;

* It is comparing the greatest with the smallest things, to observe that the time of the revolution of a planet round the sun, or the planetary year, bears the same proportion to the time in which the planet would fall to the sun, which the square of the side of a bee's cell does to one of the six triangles, or to the sixth part of the rhomboidal plate. (See Appendix to vol. i., Paley Illustrated.) 
$u=\frac{1}{\mathrm{Z}}$, and $v=\sqrt{2} \cdot \mathrm{Z}$; also $d t=\frac{d x}{v}=\frac{d x}{\sqrt{2} \cdot \mathrm{Z}}$.

Therefore $t=\sqrt{2} \int \frac{d x}{\mathrm{Z}}=\sqrt{ } \overline{2} \cdot \zeta$, or the time is as

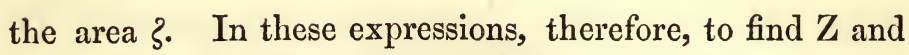
$\zeta$ we have to substitute the values of $\mathrm{X}$ and $\mathrm{Z}$ in terms of $x$, and integrate.

It is hardly necessary to add, that if, instead of the velocity and the time being sought $(Z$ and $\zeta)$, these are given, and the place reached by the body be sought, we find it by the same construction; and ascertaining what value of $x$ gives the value of $Z$, the square root of the area. But it may be well to note here, that if $\mathrm{O} M$ be the curve, whose ordinate $\mathrm{P} \mathrm{M}$ or $y=\mathrm{X}$, the centripetal

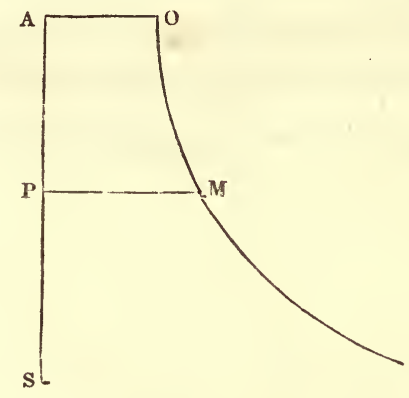

force at $\mathrm{P}$ in terms of $\mathrm{A} \mathrm{P}$ or $x$, or the gravitation of any particle of a homogeneous fluid towards $\mathrm{S}$ at the point $\mathrm{P}$; then the column of that fluid whose altitude is $\mathrm{A} P$ will press at $\mathrm{P}$, as the area $\mathrm{A} P \mathrm{M} \mathrm{O}$, or as $v^{2}$, the square of the velocity acquired by a body falling through $\mathrm{A} P$.

iv. The next object of research is to generalise the preceding investigations of trajectories from given forces, and of motion in given trajectories, applying the inquiry to all kinds of centripetal force, and all trajectories, instead of confining it to the conic sections, and to a force inversely 
as the square of the distance. This forms the subject of the Eighth Section, which therefore bears to the Third, Fourth, Fifth, and Sixth, the same relation that the concluding investigation of the Seventh Section (on rectilinear motion influenced by centripetal force) bears to the rest of that section.

The length at which we before went into the solution of the problem of central forces (inverting somewhat the order pursued in the Principia) makes it less necessary to enter fully into the general solution in this place. We formerly gave the manner of finding the force from the trajectory in general terms, and showed how, by means of various differential expressions, this process was facilitated. It must, however, be remarked, that the inverse problem of finding the trajectory from the force, is not so satisfactorily solved by means of those expressions. For example, the most general one at which we arrived of $\frac{\sqrt{y^{2}+(x-a)^{2}} \times d x^{2} \cdot d \mathrm{X}}{2(y d x-(x-a) d y)^{3}}$ being put $=\frac{\mathrm{C}}{y^{2}+(x-a)^{2}}$, or the force inversely as the square of the distance, presents an equation in which it may be pronounced impossible to separate the variables so as to integrate, at least while $d \mathrm{X}$, the differential of $\frac{d y}{d x}$, remains in so unmanageable a form; for then the whole equation is $\frac{d^{2} y d x-d^{2} x d y}{2(y d x-(x-a) d y)^{3}}$ $=\frac{\mathrm{C}}{\left(y^{2}+(x-a)^{2}\right)^{\frac{3}{2}}}$, and thus from hence no equation to the curve could be found. It cannot be doubted that Sir Isaac Newton, the discoverer of the calculus, had applied all its resources to these solutions, and as the expressions for the central force, whether $\frac{r}{2 p^{3} \cdot \mathrm{R}}$, or $\frac{d p}{p^{3} d r}$, or 
$-\frac{d^{2} x \sqrt{ } x^{2}+y^{2}}{x}$ (in some respects the simplest of all, being taken in respect of $d t$ constant, and which is integrable in the case of the inverse squares of the distances, and gives the general equation to the conic sections with singular elegance), are all derivable from the Sixth Proposition of the First Book, it is eminently probable that he had first tried for a general solution by those means, and only had recourse to the one which he has given in the Forty-first Proposition when he found those methods unmanageable. This would naturally confirm him in his plan of preferring geometrical methods; though it is to be observed that this investigation, as well as the inverse problem for the case of rectilinear motion in the preceding section, is conducted more analytically than the greater part of the Principia, the reasoning of the demonstration conducting to the solution and not following it synthetically.

$A$ is the height from which a body must fall to acquire the velocity at any point $\mathrm{D}$, which the given body moving in the trajectory V I K (sought by the investigation) has at the corresponding point I; D I, E K, being circular arcs from the centre $\mathrm{C}$, and $\mathrm{C} \mathrm{I}=\mathrm{C} \mathrm{D}$ and $\mathrm{C} \mathrm{K}=\mathrm{C} \mathrm{E}$. It is shown previously that, if two bodies whose masses are as their weights descend with equal velocity from $A$, and being acted on by the same centripetal force, one moves in V I K and the other in A V C, they will at any corresponding points have the same velocity, that is at equal distances from the centre C. So that, if at any point D, $\mathrm{D} b$ or $\mathrm{D} \mathrm{F}$ be as the velocity at $\mathrm{D}$ of the body moving in $\mathrm{A} \mathrm{V} \mathrm{C}, \mathrm{D} b$ or $\mathrm{D} \mathrm{F}$ will also represent the velocity at $\mathrm{I}$ of the body moving in $\mathrm{V} \cdot \mathrm{I} K$. Then take $\mathrm{D} \mathrm{F}=y$ as the centripetal force in $\mathrm{D}$ or $\mathrm{I}$ (that is, as any power of the distance $\mathrm{D} \mathrm{C}$, or $a-x, \mathrm{~V} \mathrm{C}$ being $a$, and $\mathrm{C} \mathrm{D}, x$ ) 
$\frac{\mathrm{Q} d x}{x \sqrt{f y d x-\frac{\mathrm{Q}^{2}}{x^{2}}}} ;$ and multiplying by $x, \frac{x^{2} d z}{a}$ (twice the sector I C K $)=\frac{\mathrm{Q} d x}{\sqrt{\int y d x-\frac{\mathrm{Q}^{2}}{x^{2}}}}$. Again $a d z: \frac{x^{2} d z}{a}$ $:: a^{2}: x^{2} ;$ and $a d z=\frac{x^{2} d z}{a} \times \frac{a^{2}}{x^{2}}=\frac{a^{2}}{x^{2}} \times$ $\frac{\mathrm{Q} d x}{\sqrt{\mathrm{Q}^{2}}}=$ twice the sector $\odot \mathrm{Y} \mathrm{CX}$. $\sqrt{f y d x-\frac{\mathrm{Q}^{2}}{x^{2}}}$
Hence results this construction. Describe the curve $a b \mathrm{Z}$, such that $(\mathrm{D} b=u)$ its equation shall be $u=\frac{\mathrm{Q}}{{ }_{2} \sqrt{\int y d x-\frac{\mathrm{Q}^{2}}{x^{2}}}}$ and the curve $a c x$ such that (D $c=\phi)$ its equation may be $\phi=\frac{\mathrm{Q} a^{2}}{2 x^{2} \sqrt{\int y d x-\frac{\mathrm{Q}^{2}}{x^{2}}}}$ Then the differentials of the areas of these curves, or $u d x$ and $\phi d x$, being respectively $\frac{\mathrm{Q} d x}{2 \sqrt{\int y d x-\frac{\mathrm{Q}^{2}}{x^{2}}}}$ and $\frac{\mathrm{Q} a^{2} d x}{2 x^{2} \sqrt{\int y d x-\frac{\mathrm{Q}^{2}}{x^{2}}}}$ and those being equal to $\frac{x^{2} d z}{2 a}$ and $\frac{a d z}{2}$, or the sectors which are the differentials of the areas V I C and V X C, the areas themselves are equal to those areas; and therefore from $\mathrm{V} \mathrm{X} \mathrm{C} \mathrm{being} \mathrm{given} \mathrm{(if} \mathrm{the} \mathrm{area} c \mathrm{D} \mathrm{V} \boldsymbol{a}$ be found), and the radius $\mathrm{C} \mathrm{V}$ being given in position and magnitude, the angle $\mathrm{V} \mathrm{C} \mathrm{X}$ is given; and from $\mathrm{C} \mathrm{X}$ being given in position, and $\mathrm{C} \mathrm{V}$ in magnitude and position, and also the area $\mathrm{CIV}$, (if $\mathrm{VD} b a$ be found), the point $\mathrm{I}$ is found, and the curve VIK is known. This, however, depends upon the quantities made equal to $u$ and $\phi$ 
severally being expressed in terms of $x$, for this is necessary in order to eliminate $y$ from the equations to these curves; and then it is necessary to integrate these expressions; for else the angle V C X, and the curve V I K, are only obtained in differential equations. Hence Sir Isaac Newton makes the quadrature of curves, that is, first the integration of $f y d x$, to eliminate $y$, and then the integration of the equations resulting in terms of $u$ and $x, \phi$ and $x$ respectively, the assumptions or conditions of his enunciation.-The inconvenience of this method of solving the problem gave rise to the investigations of Hermann and Bernouilli. The equation of the former, involving, lowever, the second differential of the co-ordinate, is to the rectangular co-ordinates; that of the latter is a polar equation, in terms of the radius vector and angle at the centre of forces.

To illustrate the difficulty with which this method of quadratures is applied, in practice - take the case of the centripetal force being inversely as the cube of the distance; then $y=\frac{\mu}{x^{3}}$ and the curve $\mathrm{BLF}$ is quadrable. If we seek the circle V X Y by rectangular co-ordinates $\mathrm{X} \mathrm{O}, \mathrm{O} \mathrm{V}$, we find the equation to obtain $\mathrm{OV}=\mathrm{D}$ in terms of $x$, is of the form

$$
\begin{aligned}
\frac{1}{2} \frac{a^{2} d \mathrm{D}}{\sqrt{2 a \mathrm{D}-\mathrm{D}^{2}}}= & \frac{\mathrm{Q} a^{2} d x}{2 x^{2} \sqrt{\int y d x-\frac{\mathrm{Q}^{2}}{x^{2}}}} \\
& =\frac{\mathrm{Q} a^{2} d x}{\sqrt{2} x \sqrt{2 c x^{2}-\mu-2 \mathrm{Q}^{2}}}
\end{aligned}
$$

( $c$ being the constant introduced by integrating $\int y d x$ ). Now there is no possibility of integrating these two quantities otherwise than by sines, and we thus obtain, nor can we do more, the following equation to $\mathrm{D}$ in terms of $x$; 


$$
\begin{gathered}
\mathrm{C}-a^{2} \arcsin \frac{a-\mathrm{D}}{a}= \\
\frac{\sqrt{2} \cdot \mathrm{Q} \cdot a^{2}}{\sqrt{\mu+2 \mathrm{Q}^{2}}} \times \arccos \cdot \frac{\sqrt{\mu+2 \mathrm{Q}^{2}}}{\sqrt{2 c} \cdot x} .
\end{gathered}
$$

And if we get $\mathrm{D}$ from this, in terms of cos. $x$, we have then to obtain P C by similar triangles, and from I P C being right-angled and $\mathrm{I} \mathrm{C}=x$, to obtain $\mathrm{P} \mathrm{I}$, in order to have the curve V I K.

But if we proceed otherwise, and instead of working by quadratures, take $v$ the velocity of the body at $\mathrm{I}$, or in the straight line at $\mathrm{D}$, and make $\frac{c}{4}$ the area described in a second, and $\theta$ the angle VCI, we obtain as a polar equation to V I K, $d \theta=\frac{c d x}{x \sqrt{4 x^{2} v^{2}-c^{2}}}(x$ being in this case both $\mathrm{CD}$ and the radius vector). Then, to apply this general equation to the case of the centripetal force being as $\frac{1}{x^{3}}$, let the force at the distance 1 be put equal to unity, and supposing the velocity of projection to be that acquired in falling from an infinite height, the equation to the trajectory becomes

$$
\begin{aligned}
d \theta=\frac{c d x}{x \sqrt{4-c^{2}}}, \text { and integrating, } \theta= & \frac{c}{\sqrt{4-c^{2}}} \\
& \times \log \cdot \frac{x}{a} .
\end{aligned}
$$

The whole subject of centripetal forces, inverse and direct, under the four heads which we began by stating, has therefore been discussed, but always upon the assumption that the bodies acted upon move in orbits which remain at rest, and thus that the axis of the curve which they describe remains constantly in the same position. Another subject of inquiry is presented to us if that axis 
itself moves, revolving round the centre of forces, and we are required to ascertain the line in which the body moves in this moving orbit, as related to the line described by a body moving in a fixed orbit; or conversely to ascertain the motion in the two orbits. This subject divides itself into two branches, according as the planes in which the motions are performed pass through the centre of forces or not. Motions in the planes of the centre form the subject of the Ninth Section; the Tenth treats of motions in eccentric planes. Under the former division, a principal object of investigation is that which indeed measures the orbit's motion, and is identical with it, the motion of the apsides; in other words, the positions successively taken by the two points of the revolving orbit, where the tangents are perpendicular to the axis, and where, consequently, the moving body begins to come back towards the centre from its greatest distance in that direction of the axis; while, under the latter division of the subject, a main point of discussion is the vibration of pendulums.

i. If a body, revolving round a centre of forces, is acted upon laterally by any other force beside the centripetal and the centrifugal (or tangential), though the centre may remain fixed, the orbit will not remain so. The axis of the curve described will move forward or backward, according to the direction of the disturbing force. This motion of the axis is considered as a revolving motion of the orbit, and is the subject of our present consideration. The great practical importance of the inquiry will presently be shown. Suppose a body moves in an ellipse that is very nearly a circle, the centripetal force being inversely as the square of the distance; the centrifugal force is in the direct proportion of the square of the velocity and the inverse proportion of the distance, jointly; that is, ( $a$ being the distance, 
and $v$ the velocity in a circle,) as $\frac{v^{2}}{a}$; and $v$ being as $\frac{1}{a}$, the centrifugal force is as $\frac{1}{a^{3}}$, or inversely as the cubes of the distances. $\mathrm{ACB}$ is the fixed ellipse; $a \mathrm{C} b$, the one described by the body under the influence of a disturbing force, or in any other way made to move in an orbit whose axis, $b \mathrm{~S}$, or line joining the apsides $a$, $b$, is revolving round $\mathbf{S}$. Suppose the angular motion

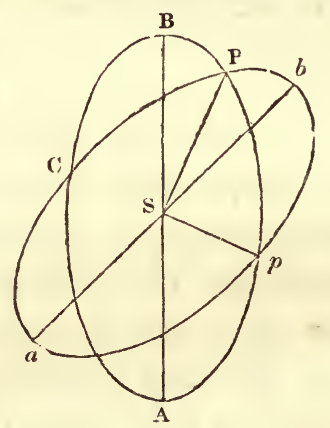

of the second ellipse to be in a given proportion to the motion of the body in the first, or that $s p$ being equal to $\mathrm{SP}$, the angle $\mathrm{BSP}$ is $\frac{m}{n}$ of the angle $b \mathrm{~S} p$. The difference of the centrifugal forces of the two bodies must be equal to the difference of their centripetal forces. Calling $\mathrm{T}$ and $t$ the centrifugal forces in the fixed and moveable orbits respectively; $\mathrm{C}$ and $c$ the centripetal forces; $\mathrm{T}-t=\mathrm{C}-c$, and $c=\mathrm{C}+t$ - T. But $\mathrm{T}: t$ in the proportion of the squares of the velocities, or of the angular motions, that is as $m^{2}: n^{2}$ and $\mathrm{T}-t: t:: m^{2}-n^{2}: n^{2}$; and because the centrifugal forces are at different distances, inversely as the cubes of those distances, therefore the difference of those forces in the two orbits, being in a given ratio to either 
of them, must be inversely as the cube of their common distance from the centre, or of the altitude of the revolving body in its orbit. Hence it follows that $d$ being the common altitude or distance, and $\mathrm{P}$ the parameter, the force required to move the body in the moveable ellipse is as $\frac{m^{2}}{d^{2}} \times \frac{\mathrm{P}\left(n^{2}-n^{2}\right)}{2 d^{3}}$; and, conversely, if such is the force, the motion will be in a moveable ellipse: And again, if $a$ be the transverse axis of the ellipse, the forces in the fixed and in the moveable orbit will be to each other as $\frac{m^{2} d}{a^{3}}$ and $\frac{m^{2} d}{a^{3}}+\mathrm{P} \frac{\left(n^{2}-m^{2}\right)}{2 d^{3}}$. Hence, in order that a body may move in a moveable ellipse, or an arc which advances or moves round in the direction of the body's motion, the centripetal force must vary in a higher proportion than the inverse square of the distance, but less than the cube; and that the body may move in a retiring ellipse, or an arc which moves round in a direction contrary to that of the body's motion, the centripetal force must vary in a less proportion than the inverse square of the distance.

From these propositions, Sir Isaac Newton is enabled to ascertain the proportion of the centripetal force to the distance, when the motion of the elliptical axis, that is of the apsides, or extreme points of it, shall be given; and conversely to ascertain the motion of the apsides when the proportion of the centripetal force to the distance is given. Let $\phi: \theta$ be the proportion of the angular motion by which the body in the moveable orbit comes round to the same lines of apsides, to the angular motion of one revolution, or $360^{\circ}$; then the centripetal force will be as the power of the distance $d$, which is represented by $\frac{\phi^{2}}{\theta^{2}}-3$. Thus, if $\phi=\theta$, or the axis of the move- 
able orbit moves only through the same space with the axis of the fixed orbit, that is if the moveable orbit coincides with the fixed, then the centripetal force is as $d^{1-3}=d^{-2}=\frac{1}{d^{2}}$; and conversely, if the central force is as $\frac{1}{d^{2}}$, the line of the apsides has no motion whatever. Hence the important proposition, that the inverse square of the distance, the actual law of gravitation, is the only proportion which prevents the line of the apsides from moving at all.—Again, if $\phi: \theta:: 363: 360$, or the line of the apsides advances three degrees in each revolution, then the centripetal force is between the inverse square and inverse cube of the distance, but much nearer the former; for $\frac{\theta^{2}}{\phi^{2}}-3$ becomes nearly equal to $2 \frac{4}{2} \frac{3}{3}$, or about $2 \frac{1}{61}$. But suppose the excess of the angle between the axes in one orbit over that angle in the other orbit to be only $11^{\prime \prime} 53^{\prime \prime \prime}$, * then $\frac{\theta^{2}}{\phi^{2}}-3$ becomes equal to $-2 \frac{1}{\overline{5} \frac{1}{9} \overline{5}}$, or say $-2_{\overline{6} \frac{1}{0} \overline{0} \overline{0}}$, and the force as $\frac{1}{d^{2 \frac{1}{60000}}}$. In like manner, if some extraneous force is impressed upon the revolving body, from knowing the amount of that force we can find the motion of the apsides, and conversely. It is found by following the method of Sir Isaac Newton, that the advance in a single revolution on the supposition of the disturbing force being to the centripetal force as 1 to $357 \cdot 45$, is equal to $1^{\circ} 31^{\prime} 28^{\prime \prime}$.

Now it is found that in the planetary motions these

* The amount of $12^{\prime \prime}$ is often given for the advance of the axis of the earth's orbit; but we have followed Laplace's number of $36^{\prime \prime} 7^{\prime \prime \prime}$, which on the sexagesimal scale is $11^{\prime \prime} 89^{\prime \prime \prime}$, or $11^{\prime \prime} 53^{\prime \prime \prime}$. This small difference makes a difference of 1000 years in the total revolution. 
variations of the centripetal force actually take place. The action of the sun, for example, upon the moon, while she is acted upon by the earth, coincides with that of the earth in some parts of her orbit, and in some parts opposes this action, thus alternately adding to and taking away from the force of her gravitation towards the earth; and this increase and diminution is greater at the greater distances of the moon from the earth. Hence the proportion of the centripetal force which keeps her in her orbit, is somewhat different from the exact ratio of the inverse square of the distance. There is more taken away from this centripetal force by the sun's action while the bodies are placed towards each other in one direction, than there is added when in the other position; and therefore there is a total diminution of the moon's gravitation, or the centripetal force decreases in a somewhat higher ratio than as the square of the distance increases;

in other words, the denominator of the expression $\frac{1}{d^{2}}$ is

greater than this exact power of $d$, which we have seen keeps the orbit and its axis fixed with respect to the centre, which in this case is the centre of the earth. Hence this axis of the moon's orbit revolves in the direction of the moon's motion, and in a certain period makes a complete revolution. So that at one time, half this period, the moon's greatest and least distances from the earth (her apogee and perigee) have changed places, and at the end of the period they resume their former position. The amount of this motion of her apsides is about $3^{\circ}$ in each revolution, or $39^{\circ}$ in a year; so that the axis of her ellipse revolves in nine years;

and the centripetal force is not as $\frac{1}{d^{2}}$ but $\frac{1}{d^{2 \frac{1}{6} \mathrm{~T}}}$, nearly 
the proportion above shown to belong to a progression of the apsides, equal to $3^{\circ}$ in a revolution. In like manner the orbit of the earth is not immoveable, owing to the disturbing forces of the larger planets, Jupiter, Saturn, Mars, and Venus. But the disturbance here is, of course, incomparably more minute. The apsides of the earth's orbit only move $11^{\prime \prime} 53^{\prime \prime \prime}$ in the year, instead of $39^{\circ}$; and the expression for the centripetal force is therefore, as we have seen above, the inverse not of $d^{2 \frac{1}{61}}$, but of $d^{2 \frac{1}{60000}}$. The axis of the earth's orbit thus revolves in a period of about 109,060 years.

It is, however, to be observed that, although this motion of the axis of the earth's orbit is the result of the theory of gravitation, and indeed affords a new proof of it, Sir Isaac Newton did not himself consider it as worthy of attention. He regarded it as indicating so very minute a deviation from the law of the inverse square of the distance, as not to alter sensibly the form and position of the orbits resulting from thence. He therefore did not give any calculation respecting it. To say that he was ignorant of it, or that he affirmed the absolute quiescence of the planetary apsides, as some have done *, is wholly erroneous. The statements and methods in the Forty-fifth proposition and its corollaries are quite general, applying to all bodies acted on by disturbing forces; so is the Sixty-seventh, with the Sixth, Seventh, and Eighth corollaries, of general application; and even in the proposition (the Fourteenth of the Third book) in which he affirms that the aphelia and nodes of the orbits are at rest, he refers to inequalities arising from disturbing forces, while in the scholium that immediately follows he expressly states the motion of the aphelion of 
Mars, and collects from thence that of the Earth, Venus, and Mercury, by the law which regulates the motion of the apsides, namely, the sesquiplicate proportion to the distances. By this he makes the motion of the Earth's aphelion $17^{\prime} 40^{\prime \prime}$ in a century, or $10^{\prime \prime} 36^{\prime \prime \prime}$ yearly, being not a second and a half different from what it is now understood to be.

The calculation of the motion of the moon's apsides, however, which he deduced from these propositions, differed widely from the truth. He made it, as we have seen, amount to little more than a degree and a half each revolution*, or about one-half of the truth; and for the discrepancy between the theory and the phenomena he seems to have failed in accounting. Others, in the earlier part of the eighteenth century, having applied to the subject a different investigation, but founded upon his principles, obtained a different result, but erring by excess; for they made the motion $3^{\circ} 27^{\prime}$ each revolution, or nearly $45^{\circ}$ in the year instead of $39^{\circ}$. About the year 1745 the three great mathematicians of that age, Clairaut, Euler, and D'Alembert, investigated the subject; and applying the whole resources of analysis to its discussion as a case of the problem of three bodies, obtained general solutions of great beauty. However, they still found the theory differ with the fact nearly as much as Newton himself had done; and Clairaut was even driven by this to devise a new law for the purpose of explaining the apparent discrepancy. He supposed the centripetal force to be not as $\frac{1}{d^{2}}$ but as $\frac{1}{d^{2}}+\frac{1}{d^{4}}$. In a very short time, however, he candidly gave up this theory, and announced the important fact that he had found the whole error to arise from his hav- 
ing in his approximation neglected some quantities as extremely minute, and supposing they could not affect the result, whereas one of the quantities upon which the result mainly depends, having a small numerator, is nearly doubled by the introduction of the quantities omitted. Upon again going through the investigation without those omissions, this great geometrician had the satisfaction of finding that the result made the motion of the moon's apsides agree with the fact; and both Euler and D'Alembert now found that in their solutions they had, by a singular coincidence, fallen into the same error. Laplace has since in his great work* given a complete investigation of the problem, and the results to which he is conducted by the theory are also most satisfactory. He finds the amount to differ only one four hundred and forty-fourth part from that given by observation, which, reduced to our sexagesimal degrees, is only a difference of $24^{\prime \prime} 12^{\prime \prime \prime}$ from the observed amount. His solution in the case of the nodes does not come so near the observation; it is only correct within the 350th part; and yet the success of the theory in the case of the nodes was always reckoned its great victory in the hands of its author, while the case of the apogee cast some doubt upon it. Laplace made a discovery in the course of this inquiry of a similar variation in the apogeal movement, and that it becomes slower at the rate of $15^{\prime \prime}$ in 100 years, which the recent observations confirm.

It was certainly impossible for the Newtonian theory to obtain a more brilliant triumph. $\dagger$ But it deserves to

* Méc. Cél. liv. vii. s. 16.

† For Clairaut's papers, see Mém. de l'Acad. des Sciences, 1745 and 1748. But there is an admirable paper of the same illustrious mathema. tician on the motions of the orbits in the Mém. for 1754. The first cited volume contains both Clairaut and D'Alembert's famous investigation of the problem of the three bodies, to which reference is made in the text 
be mentioned, that the statement made by Bailly is even more incorrect upon this subject of the moon's apsides than upon the motion of the planetary axis. He asserts that Newton represented the theory as "giving the quantity of the moon's apogeal motion with exactness;" and that this having been a mere dictum of his without a demonstration, philosophers waited to find it proved by subsequent inquiry, as the theory had been on so many other points. The great inaccuracy of the substance is assuredly not rendered the less distasteful by the manner of this statement. "Il avait souvent parlé à la manière des prophètes qui disent ce qu'on ne peut voir: alors c'est la foi qui croit, il faut que la raison se soumette." (Hist. de I Astron. iii. 150.) Newton never asserts anything which may not, from what he himself lays down, be strictly demonstrated. He certainly leaves much to be supplied; but he never leaves the reader who would, with due knowledge of the mathematics, follow his reasoning, to trust his word. Even the scholium at the close of the Lunar theory (after Proposition xxxv. B. iii.), where more of the investigation is omitted than perhaps in all the rest of the Principia together, may be followed argumentatively by a learned and diligent reader, as the Jesuits have shown in their inimitable commentary upon it. But touching the particular instance referred to by Bailly, nothing can be more contrary to the fact than his statement. Sir Isaac Newton in the general proposition which we have analysed above, after finding that any body acted upon by a disturbing force in the given proportion to the centripetal, will have by the theory a progressive motion of its apsides equal to $1^{\circ} 31^{\prime} 28^{\prime \prime}$, although he had not in the whole

as haring been undertaken by them and Euler at the same time.-See Life of D'Alembert, p. 427., where the history of this celebrated investigation. is giren at length. 
corollary made any particular application to the moon's motion, adds, "the apsis of the moon has a velocity twice as great nearly" (apsis lunæ est duplo velocior circiter), (Cor. 2. to Prop. xlv. B. i.) ; ${ }^{*}$ and though in the proposition in which he applies his theory to find the disturbing force of the sun, (xxv. B. iii.) he finds it to be to the centripetal force as 1 to $178 \frac{1}{2}$ nearly (or double what he had argued upon in the former proposition), he is so far from deducing from thence any inference that the apsides by the theory move $3^{\circ}$ in each revolution, that he makes no application at all of the proposition to finding their motion. But in the celebrated scholium where he sums up all the disturbances, he treats of this motion, and he expressly shows that it only comes out to be anything like the true motion of $3^{\circ}$ by an assumption contrary to the theory; that is, by taking not the true equation to the sun's mean motion, but the equation on the hypothesis of its following the inverse triplicate ratio. The words above quoted from the general proposition upon the apsides in the first book, are quite sufficient to protect Newton's memory from any such aspersion as that now under consideration.

It may further be remarked, that Bailly's general criticism on Newton's whole investigation of the moon's motion is singularly unfortunate. He represents him as having only given a rough sketch of the subject, leaving others to fill up. He says, that this is the part of Newton's work most involved in obscurity; that, concealing the route he pursued, he plainly has not taken the problem in its full extent, but only shown generally, and by a few examples, that those irregularities could be deduced from the theory; though he renders

* It is remarkable that these words are not in the first edition of the Principia. 
ample justice to Newton's transcendent merits in other respects. But here Bailly has a far higher authority than his own against him, justly as his own name is held in respect. Laplace, in his earlier writings*, had seemed not sufficiently impressed with the inestimable value of that part of the Principia; and had, while he distinctly gave the work at large the "pre-eminence over all other productions of the human understanding," yet appeared to regard the theory of disturbed planetary motion, and especially of the moon's motion, as a sketch left for others to complete when the calculus should be more improved. Yet in his last work, the concluding part of the Mécanique Céleste, published the year before his death, he distinctly declares this very portion of the Principia to be among the greatest monuments of the author's genius. "Je n'hésite point à les regarder (recherches sur la théorie de la lune) comme une des parties les plus profondes de cet admirable ouvrage." $\dagger$

It remains, however, that we mention an unaccountable statement of the truly great geometrician whom we have last cited. In treating of the history of the lunar theory, he says that Newton, when seeking the correction of the sun's disturbance of the moon's gravitation towards the earth, "supposes that disturbance to be $\frac{2}{3} \frac{1}{5} 7$ of the moon's gravity, or that which results from the observed amount of the lunar apogee." (Méc. Cél. lib. xv. chap. 1.) For this he refers to Book iii. Prop. iv. of the Principia, which is evidently a wrong reference, that proposition, and indeed that part of the book, treating of other subjects. Nor can any place be found that Laplace could have had in his view, except the Twenty-first proposition of the Third

* Système dn Monde, liv. v. chap. 5.

† Méc. Cél. liv. xvi. chap. 1.; published in 1825. 
book, in which the sun's disturbing force on the moon's motion is investigated. But respecting that proposition, it is wholly inaccurate to say that he there makes any hypothesis or assumption of the proportion between the disturbing force and the moon's gravity; for he deduces the proportion of 1 to $178 \frac{2}{4} \frac{9}{0}$, (or which is nearly the same thing, 2 to 357 ,) from the duplicate ratio of the periodic times, and deduces it as a consequence of the Seventeenth corollary to the Sixty-sixth proposition of the First book, which corollary comes easily from the Second corollary of the Fourth proposition of the First book. It is, therefore, wholly impossible to represent that position as a mere assumption to suit the observation of the moon's actual variations.

ii. The next subject of consideration is the motion of bodies along given surfaces, not in planes passing through the centre of forces, to which case our inquiries have hitherto been confined.

Let a body move in any plane, in a trajectory, by a force

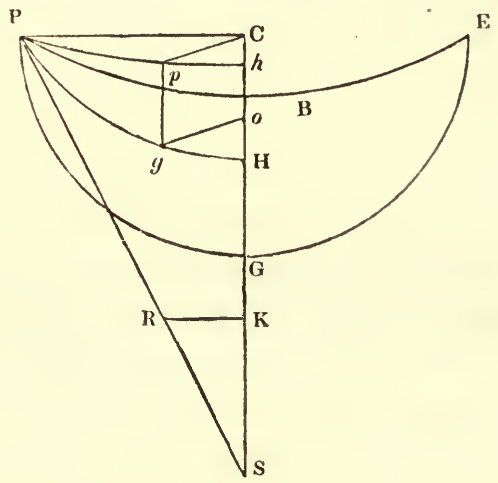

directed towards a centre out of that plane, and we are to examine its motion under two heads, as we did the motion of a body when the centre was in the plane of the trajectory; that is, first, the curve described by the given force; 
and next the force, with the velocity, when the curve is given.

For this purpose, let $\mathrm{P}$ be the perpendicular $\mathrm{S} \mathrm{C}$ to the plane from $S$ the given centre, this being the shortest line from the point to the plane; $\mathrm{D}$ the distance $\mathrm{S} \mathrm{P}$ from the centre to any point $\mathrm{P}$ of the curve; the distance $\mathrm{C} \mathrm{P}=d$ of that point $\mathrm{P}$ to the centre in the plane, that is, to the point $\mathrm{C}$ where $p$ falls on the plane; and let $\mathrm{F}$, the central force, be represented by $\mathrm{R} \mathrm{S}$. It is evident that the force $\mathrm{R} \mathrm{S}$, acting in the line $\mathrm{P} \mathrm{S}$ (without the plane), is compounded of two, $R K$ and $S K$, of which $R K$ only can have any effect on the motion in the plane, the other $\mathrm{S} \mathrm{K}$ which tends to draw the body out of the plane being by the supposition nothing, because the body moves wholly in the plane C P B E. But by similar triangles, $R \mathrm{~K}=\frac{\mathrm{S} \mathrm{R} \times \mathrm{C} \mathrm{P}}{\mathrm{P} \mathrm{S}}$ $=\frac{\mathrm{F} \cdot d}{\mathrm{D}} ;$ therefore if the proportion of the centripetal force to the distance be known, that is, if $\mathrm{F}=\mathrm{D}^{n}, \mathrm{RK}=d$. $\mathrm{D}^{n-1}$. But $\mathrm{D}^{2}=d^{2}+p^{2}$, and $\mathrm{D}=\sqrt{d^{2}+p^{2}}$; therefore $\mathrm{R} K$, the force acting at $\mathrm{P}$ towards the centre $\mathrm{C}$, is $d \times$ $\left(d^{2}+p^{2}\right) \frac{n-1}{2}$, which gives it in terms of the distance $\mathrm{C} \mathrm{P}$, and the given line SC. Thus if the central force is as the distance $\mathrm{SP}$, the force acting towards the centre becomes equal to $d$, or as the distance on the plane. So if the central force is inversely as the distance, then $n=-1$, and the force to the centre on the plane is $\frac{d}{\mathrm{D}^{2}}$ or $\frac{d}{d^{2}+p^{2}}$, and if it is inversely as the square of the distance, the force on the plane is $\frac{d}{\left(d^{2}+p^{2}\right)^{\frac{3}{2}}}$. But the central force being given in the plane, the investigation 
is reduced to that formerly explained, for finding motions and trajectories when the centre is in the same plane with the motion. Hence in the case first put, of the force towards $\mathrm{S}$ being as $\mathrm{D}$, and the force towards $\mathrm{C}$ being, consequently, as $d$, it follows from what was formerly shown respecting motion in the same plane, that the curve described on the plane of the centre $\mathrm{C}$, or $\mathrm{P} \mathrm{B}$, in this case is an ellipse; that the times in which the ellipse is described will be the same in whatever plane the bodies move; and that if the ellipse, by lengthening its axis indefinitely, becomes a straight line, the vibrations of the body in that line will be performed in equal times to and from the centre on both sides of it.

By a somewhat similar process, we find the motion and trajectory of a body moving on a curve surface, by a force directed towards a given centre in the axis of the solid of revolution which forms that curve surface. It is first shown, that if from any point of the trajectory $\mathrm{P} g \mathrm{H}$ on the curve surface (which being a curve of double curvature we shall call the double curve), a perpendicular $g o$ be drawn to the axis C S, and from any other point of the axis there be drawn a line equal and parallel to $g o$, as $\mathbf{C} p$, $\mathrm{C} p$ will describe areas proportional to the times. By means of this proposition and the former ones respecting motion in the same plane, we are enabled to find the curve $\mathrm{P} p h$ on the plane P B E, the points of which curve are, as it were, a projection on that plane of the trajectory, or double curve, $\mathrm{P} g \mathrm{H}$; and having found $\mathrm{P} p h$, the double curve is found by drawing perpendiculars to the plane $\mathrm{P} \mathrm{B} \mathrm{E}$, from the curve $P p h$ to the curve surface $\mathrm{P} \mathrm{G} \mathrm{E}$, whose form is given. Thus suppose the solid to be a cylinder, in which case the curve $\mathrm{P} p h$ will be the circle which is the section of the cylinder; then if the central force acts (by $\mathrm{S}$ being removed to an infinite distance) in lines 
parallel to the axis, and we suppose the body to begin its motion in the double curve $\mathrm{P} g \mathrm{H}$, with the same velocity as that given or central velocity, with which it would describe $\mathrm{P} p h$, the double curve is found by taking the ordinates $p g$ in a given proportion to the square of the circular $\operatorname{arch} \mathrm{P} p$, or as $\frac{\mathrm{P} p^{2}}{m}$; and consequently $\mathrm{P} g \mathrm{H}$ is a species of quadiatrix described on a cylinder.

The motion of pendulums is evidently a case of motion in a curve surface by a force directed towards a point in the axis of the solid, of which solid the curve described by the pendulous body is a section; and Sir Isaac Newton discusses this subject fully. As subservient to this inquiry, he gives some important properties of the cycloid, or rather of the hypocycloid and hypercycloid: For he is not satisfied with the investigation, which is sufficiently easy, of the ordinary cycloid's properties, the curve described by a point in a circle or wheel running along a straight line, but examines what is more difficult, the properties of the hypercycloid and hypocycloid, or the curves described by a wheel moving on the convex, and the concave great circle of a sphere respectively. Of these properties the most important is this. If $\mathrm{D}$ be the diameter of the sphere, and $d$ that of the wheel, the length of the hypercycloid is equal to four times $\frac{d}{\mathrm{D}} \times(\mathrm{D}+d)$, or four times the length of a fourth proportional to the sum of the two diameters, the wheel's diameter and the sphere's. It is then shown how a pendulum may be made to vibrate in a given cycloid, or rather hypocycloid, namely, by taking a distance, which is a third proportional to the part of $D$, which the hypocycloid cuts off (that is, the distance of the hypercycloid from the centre of the 
sphere) and $\frac{D}{2}$; and from that distance $\mathrm{S}$ so found, drawing two cycloids touching the sphere, or its great circle, and

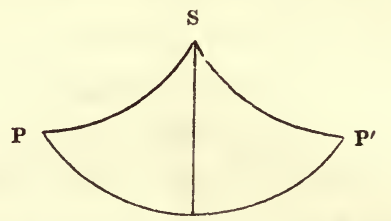

meeting in the point so found. If to that point $\mathrm{S}$, a flexible line or thread be attached and bent round one of the cycloids SP, it will unrol itself and then bind itself round the other cycloid $\mathrm{SP}^{\prime}$, and its extremity will describe the cycloidal curve $\mathrm{P} \mathrm{P}^{\prime}$ required, one of whose properties is, that all the vibrations in its arches are performed in equal times, however unequal the lengths of these arcs may be, provided that the centripetal force is in each part of the curve directly as the distance from the centre, and that no other force acts on the moving body.

But the same solution may be generalised and applied to any given curve whatever; for the curves found, and along which the flexible line is traced and from which it is then unrolled, are the evolutes of the given curve; and are found in each case by means of the radius of curvature, being the curve formed by its extremity, or the locus of the centres of the osculating circles to all the given curve's points. If the curve in which the body is to move be a circle, the evolute is, of course, a point, the centre of that circle, the radius of curvature being that of the circle. If the curve is a conic parabola, it will be found that the evolutes, or the lines from which the pendulum's thread must wind off, are cubic parabolas, whose equation is $y^{2}=\left(\frac{2}{3} x\right)^{3}$, the length of the pendulum being unity. The only case of the problem investigated by Sir Isaac Newton is that of the cycloid, which has the remarkable property, 
that its evolute is an equal and similar cycloid,-a property which it has in common with another curve, the logarithmic spiral, whose tangent makes with the radius vector a constant angle. He investigated the case of hypocycloids and hypercycloids, rather than the common cycloids, because it is that of the earth's gravity, which above the surface decreases inversely as the square of the distance from the centre, but within the sphere increases as the distance simply.

It follows, from the propositions respecting the vibration of pendulums, that the times of the descent of falling bodies may be compared together and with the times of vibrations of the pendulum: So that the time of a vibration round a given centre being given, as a second, the time of the falling body's descent to the centre of forces can be found, or the equal time of vibration in the circular arch of $90^{\circ}$ with any radius. The time is to the given time as 1 to $\frac{\sqrt{\mathrm{L}}}{\mathrm{D}}, \mathrm{L}$ being the length of the pendulum, and $\mathrm{D}$ the distance from the point of suspension to the centre of forces; and since D becomes infinite and the lines in which the central force acts parallel, and since half the length of the pendulum is to the line fallen through in the time of one vibration as 1 to 9,869 nearly (the proportion of the square of the diameter to that of the circumference), we can easily ascertain the force of gravity at any point by the length of the pendulum vibrating seconds. It is found to be in these latitudes about $34 \cdot 44$; consequently a body falls in a second through about 16 feet 9 inches.

Hitherto we have only considered the motions and trajectories of bodies acted upon by forces directed towards a fixed centre whether in the plane of their motion or out of that plane, and supposing that plane either to be fixed or to be moved round the centre of forces. But as action 
and reaction are equal and opposite, by the third of the Laws of Motion originally stated, it is evident that the case of a fixed centre cannot exist when the attraction, which we call the centripetal force, proceeds from a body placed in the centre, unless, indeed, some counteracting force shall fix this body to one point; for if no force exists but the mutual action of the two bodies, the central body must be acted upon by the one which moves round it, and its position must be affected by this action. Hence, for example, if there were only two heavenly bodies, $\mathbf{M}$ and $\mathbf{E}$, and the one, $\mathbf{M}$, moved round the other, E, by a projectile force originally impressed upon it, the other, $\mathbf{E}$, would also move round $\mathbf{M}$, unless the mass of the latter body was infinitely small, and its attraction, proportional to this mass, could not sensibly affect the larger body. Again, if two bodies, the one moving round the other, both together move round a third, $S$, the action of this third will affect the motions of the other two relatively to each other. Thus each smaller system will be affected, both as to the motions and orbits of the bodies composing it, by the action of the body in the common centre of the whole; and they will also be affected by the action of the bodies in the other systems, having the same common centre. The inquiry, therefore, divides itself into two branches; first, the difference between the motions which we have hitherto been considering when the centre was fixed, and the actual motions of the system, as that of the moon and earth revolving round each other with a moveable centre; secondly, the still more important difference between the motions already considered, and the actual motions, which difference is caused by the mutual actions of the whole bodies on each, and varies both the motions and the orbits of all.

i. Suppose two bodies mutually attracting cach other 
and impelled by a single original force of projection, as E and $M$, their centre of gravity being $G$; it is clear that

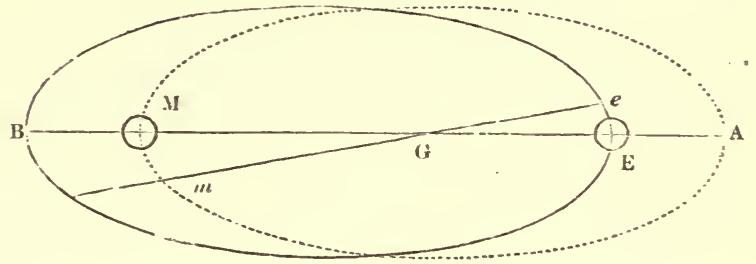

if $\mathrm{M}$ moves a very small space to $m$ by the attraction of $\mathrm{E}$, so will $\mathrm{E}$ move to $e$ by the attraction of $\mathrm{M}$, and the two triangles $\mathrm{E} \mathrm{G} e$ and $\mathrm{M} \mathrm{G} m$ will be similar in all respects; for the lines $\mathrm{M} \mathrm{G}, m \mathrm{G}$ and $\mathrm{EG}, e \mathrm{G}$ are proportional, because the segments of the lines EM and $e m$ are always in the same proportion, $\mathrm{G}$ being the centre of gravity, and those segments, therefore, inversely as the masses of $\mathrm{E}$ and $\mathrm{M}$. Therefore the curves which the bodies describe round the centre of gravity will be entirely similar. In like manner they will describe similar curves each round the other, and the radius vector of each from the other, as well as from the centre of gravity, will describe areas proportional to the times. It follows from this and from what was before shown respecting centripetal forces, that the two bodies will move in concentric ellipses round one another and round their common centre of gravity, if the centripetal force is as the distance, and that each will describe one or other of the conic sections, having the other, or the common centre of gravity, in the focus, if the centripetal force is inversely as the square of the distance. In like manner, because of the ratio between the squares of the periodic times and the cubes of the distances, it may be shown that if $\mathrm{T}$ be the periodic time of the bodics moving round their centres of gravity, and $t$ 
the periodic time of $\mathrm{M}$ moving in a similar figure round $\mathrm{E}$ at rest, $\mathrm{T}: t:: \sqrt{\mathrm{E}}: \sqrt{\mathrm{M}+\mathrm{E}}$. Further if these bodies move with forces inversely as the squares of the distances round their centres of gravity, and $\mathrm{A}$ be the greater axis of the ellipse described by $\mathrm{M}$ round $\mathrm{E}$, $a$ the greater axis of the ellipse it would describe round $\mathrm{E}$ at rest in the same time, and if $\mathrm{M}+\mathrm{E}: m:: n: \mathrm{E}$, then $\mathrm{A}: a:: \mathrm{M}+\mathrm{E}: m$. Hence, if we have the periodic times of the planets, we can find the greater axes of their orbits by taking $\mathrm{A}^{3}$ to $a^{3}$ in the proportion of $\mathrm{T}^{2}$ to $t^{2}$ (the ellipse being supposed described round the sun), and multiplying it by $\frac{n}{\mathrm{E}}$. So the masses may, likewise, be found from the distances.

The motions and paths of bodies thus mutually acting are now to be considered.-And first our author shows, that if two bodies act on each other, and move without any other, or foreign, influence whatever, their motion will be the same as if, instead of acting on one another, some third body placed in their centre of gravity acted upon each of them with the same force with which each acts on the other; and the same law will prevail (but referred to the distances from the centre) which prevailed in their mutual actions when referred to their distances from each other. Suppose the bodies M and E to attract with forces directly as their masses $\mathrm{M}$ and $\mathrm{E}$, and inversely as any power $n$ of their distances, that is, suppose their attraction to be as $\frac{\mathrm{M}}{\mathrm{D}^{n}}$, and $\frac{\mathrm{E}}{\mathrm{D}^{n}}$, and that the distances of the centre from $\mathrm{M}$ and $\mathrm{E}$ are $\mathrm{C}$ and $c$ respectively; then because $\mathrm{C}: c:: \mathrm{E}: \mathrm{M}$, and $\mathrm{C}: \mathrm{C}+c$ (or $\mathrm{D}$ ) :: $\mathrm{E}: \mathrm{E}+\mathrm{M}$, a body in the centre will attract $\mathrm{M}$ with a force as $\frac{\mathrm{E}}{\mathrm{D}^{n}}$, if it be equal to $\frac{\mathrm{E} \times \mathrm{C}^{n}}{\mathrm{D}^{n}}$, that is 
equal to $\frac{\mathrm{E}^{n+1}}{(\mathrm{E}+\mathrm{M})^{n}}$, and, in like manner, it will attract $\mathrm{E}$ with a force equal to $\frac{\mathrm{M}}{\mathrm{D}^{n}}$, if it be equal to $\frac{\mathrm{M}^{n+1}}{(\mathrm{E}+\mathrm{M})^{n}}$. If $n=2$, or the force be as the inverse square of the distance, the body placed in the centre will be equal to $\frac{\mathrm{M}^{3}}{(\mathrm{E}+\mathrm{M})^{2}}$; if $n=-1$, or the attraction be directly as the distance, the body will be in both the case of $M$ and $\mathrm{E}$ equal to $\mathrm{E}+\mathrm{M}$; and if the attraction be as the square of the distance directly, the central body will be in the two cases of the two bodies, $\frac{(M+E)^{2}}{M}$ and $\frac{(M+E)^{2}}{E}$ respectively.

Next as to the absolute trajectory of the bodies thus acting on one another, or their path in space, we have an investigation analogous to those inquiries formerly instituted where the centre of forces was fixed. For the body or bodies being known (by what we have last shown) whose mass gives at the centre the same attractions as the two bodies exercise on each other, we can determine for each of these bodies the path in which it will move, provided we know the initial direction and velocity. Thus let $m=2$ in the last expressions, we have for the mass by which $M$ is attracted towards the common centre of gravity $\frac{\mathrm{E}^{3}}{(\mathrm{M}+\mathrm{E})^{2}}$; and proceeding as was formerly shown in the case of immoveable centres, we find that if the curve described round the centre at rest be a circle, if that centre moves in a straight line, the orbit in space will be of the cycloidal kind; if the centre moves in a circle, it will be an epicycloid or hypercycloid; and if the curve be a conic parabola, the motion of the centre will change this into a cubic parabola, which will thus be the path arising from 
its parabolic motion combined with the advance of the centre of gravity. The moon in this way describes thirteen cycloidal curves in a year, and all of them concave towards the sun.

It appears then, that the orbits of the system composed of our earth and its satellite, must be considered as traced, not by either of these bodies but by their centre of gravity. While neither body describes an ellipse round the sun, but both revolve round each other and round their centre of gravity, that centre itself describes an elliptical line - a line which would be a perfect ellipse if no disturbances of another kind than those which we have been considering interfered to alter the form of the orbit. To these disturbances we now proceed.

ii. While the primary planets and their satellites are influencing each other and while the whole motion of each subordinate system round the sun, the common centre, is the elliptical orbit described by the centre of gravity of each such system, there are disturbing forces exerted on each planet by the rest, and on the motions of satellites by the action of the sun also; so that many sensible deviations take place from the motions, and from the orbits, which those bodies, both primary planets and satellites, would have, if they moved round the common centre undisturbed; that is, if they described elliptical orbits round the sun by his attraction, without any other force acting on them, except that attraction of the sun on each planet, and the attraction of each planet on its satellites. If no such disturbances existed, and the only forces that acted were the mutual actions of the primary and satellites on each other, and of the sun on the common centre of gravity of the primary and satellites, the centre would describe an cllipse round the sun, and the primary and satellites would describe ellipses round each other and round that 
centre of gravity. Such, however, is not the case; and we are now to consider the effects of the disturbance occasioned by the sun's action upon the satellites, and the disturbance occasioned by the action of the planets on one another.-This forms the subject of Sir Isaac Newton's investigations in the second branch of that section which we have been considering; an inquiry regarded by some as the most extraordinary portion of the great work which forms the principal monument of his genius. From this opinion it is difficult to withhold our concurrence; but it may be admitted that here, as in the operations for finding orbits from given forces and conversely, the great improvements of modern analysis have afforded easier and more manageable methods of investigation. That this must be true as regards the planetary disturbances, will be apparent upon a little reflection.

The grand problem in every case is to find the precise effect of a disturbing force upon the path of a given body moving by a combined centripetal and projectile force; and what has been called the Problem of Three Bodies presents the simplest case of the question, being the determination of the motions of two bodies acted upon by one another and by a third body. But though this is the simplest case of the general question, it has been found to present difficulties of the highest order; and a general and rigorous solution of it has been found to exceed the powers of the most improved analysis. In the time of Sir Isaac Newton, that analysis of which he was the inventor had not attained any thing like its greatest perfection. Hence, in grappling with the subject, he had much of the difficulty to contend with, which made him give less convenient formulas than we now possess for the solution of the other problems relating to orbits and motions. The mere improvement of the integral calculus by the advan- 
tageous approximations through series, logarithms, and the arithmetic of sines, would have afforded important facilities for these inquiries; because the solution must come always to an integration. Accordingly Euler, D'Alembert, and Clairaut, availed themselves of that improvement to investigate the problem, as we have already seen. But soon after their researches had led to the important result formerly described, a great refinement was introduced into the calculus, which bore directly upon the subject of these inquiries; and this exceedingly facilitated the solution of the problem in its more extended application. We allude to the invention of the Calculation of Variations by Euler and Lagrange.

We have in the introductory part of this Analytical View explained that this calculus enables us to examine the transition of one curve into another in certain circumstances, by showing how those lines may be found which have certain properties in relation to other lines of a different kind, and thus to investigate problems with respect to curves whose nature changes under the investigation, because the relation between their co-ordinates is variable, and is indeed the thing sought for. It is evident, therefore, that this calculus has its immediate application to the subject in question. For the effect of the disturbing force is to change at each moment the nature of the path, which, but for that force, would be described; or the inclination of orbits to one another, which, but for such disturbances, would subsist; or the position in space, which, but for the disturbance, these orbits would have. Now, those changes produced by mutual disturbances, really comprise all the effects of the disturbances on the planetary system. Thus, beside the precession of the equinoxes and the motion of the apsides and nodes, which we have just now generally stated, 
the alteration in the form of the curve includes also the change of its eccentricity, and the acceleration or retardation of the motion itself. Hence, we have at once proved that the determination of those effects which arise from disturbing forces, is in a peculiar manner the province of this new and refined analysis, the Calculus of Variations. Therefore, beside the facilities afforded by the improvement of older methods of investigation, the addition of this new instrument to our means of solving the problem has established an entirely novel method, and opened an almost unknown field of inquiry, from which the original author of all these discoveries was necessarily shut out. Instead, therefore, of minutely going over the steps of his solution, as applied to the celestial motions, we shall show the course which he pursued by demonstrating its fundamental principles; but we shall begin by stating concisely the results of the more recent investigations as affecting the science of physical astronomy, and shall reserve the fuller discussion of this subject for the account of Laplace's work.

In considering the motions of the planets and their satellites round the sun, we may first regard him as from his magnitude and distance so little affected by their attractions, that his motion is trifling, and cannot sensibly affect that of the other bodies; so that he may be viewed as at rest; and then the smaller bodies will both move round one another, and round the larger and more distant body as if he were fixed. But not only will the movement of these bodies be thus affected by their mutual actions; they will also be affected in their motions round one another by the action of the third body, the sun; and this action will disturb and alter their relative motions, as regards both their velocities, the forms, and the positions of their orbits. Thus the position of the moon's path 
round the earth is affected by the sun's attraction, that is, by her gravitation towards the sun, which combines with her gravitation towards the earth to determine her absolute motion; and both the position of the axis of her orbit (the line of apsides), and the position of the line joining the intersections of her orbit's plane with the plane of the earth's orbit (the line of nodes), are continually changing; and we have seen in a particular manner how the apsides revolve in one period of time (about nine years), and the nodes in another, (about nineteen years).

But there is a variation in the rate at which both the line of the apsides and the line of the nodes revolve. The quantity by which both of these lines advance in each year sensibly decreases; so that the period in which each effects a complete revolution becomes longer and longer. It appears that the former line revolves now $8^{\prime} 2^{\prime \prime}$ slower than in the earliest ages of astronomical observation, about $25 \frac{1}{2}$ centuries ago; the latter line only $1^{\prime} 42^{\prime \prime} 14^{\prime \prime \prime}$ - the former motion diminishing each century by $36^{\prime \prime} 41^{\prime \prime \prime}$; the latter by $7^{\prime \prime} 51^{\prime \prime \prime}$.

It is equally found that the disturbing forces accelerate the moon's motion by a very small quantity; or that she revolves round the earth in a period of about $11^{\prime \prime} 7^{\prime \prime \prime}$ shorter than she did a century ago; her angular velocity being increased between the 12 and 13,000 millionth part of her total velocity in the period of 100 years. This makes the yearly acceleration wholly insensible; and the total acceleration, or shortening of her periodic time since the creation of our species 60 centuries ago, only 11 minutes and 7 seconds, supposing it to go on as the times; but it increases in a lower proportion (probably as the cube of the times); so that its total amount is more considerable, and Laplace reckons it at about $7^{\prime} 30^{\prime \prime}$ for the last $25 \frac{1}{2}$ centuries. This acceleration had not been 
unobserved in Halley's time, and it was discussed acutely by Mayer; but its cause was first discovered by Laplace : it is the sun's action upon the moon, combined with the variation in the orbit of the earth, the eccentricity of which has been diminishing regularly, though by an extremely small quantity (only $\sqrt{.0000007667}$ of the greater axis of our orbit); so that the orbit has been slowly approaching more and more to the circular form.

It is a great proof of the usefulness of the calculus in these investigations, that this great geometrician appears to have discovered the connexion between the earth's diminishing eccentricity and the acceleration of the moon's mean motion, by the careful examination of the mere equation or algebraical expression. For the reciprocal of the semi-axis of the moon's orbit $\frac{1}{a}$, as influenced by the sun's attraction combined with the earth's, is found to be represented by an expression, which, among other terms, contains this : $-\frac{3}{4} \frac{a^{2} m^{1} e^{12}}{a^{13}}$ in which $a^{\prime}$ is the semi-axis of the earth's orbit, $m^{\prime}$ the mass of the sun, and $e^{\prime}$ the eccentricity of the earth's orbit. Consequently, as $e^{\prime}$ decreases, $\frac{1}{a}$ increases, the term being negative; and therefore $a$ itself decreases as $e^{\prime}$ decreases; in other words, the moon's orbit is diminished, and her velocity augmented, in consequence of the earth's eccentricity decreasing. But if the diminution of the greater axis is not admitted as necessarily lessening the orbit, we may recollect the relation between the times and the mean distances, the squares of the former being as the cubes of the latter; and the mean motion is, of course, inversely as the periodic time. - However Laplace furnishes us with a still closer reason, and illustrates the 
use of the calculus, as it were, by a new triumph, in another part of the Mécanique Céleste.* For the equation of the mean angular motion is shown to be $n=\frac{t \sqrt{\mu}}{a^{\frac{3}{2}}}$, $t$ being the time, $a$ the transverse axis, and $\mu$ the sum of the masses of the two bodies, in this case the moon and the earth. Therefore $n$, the mean motion, must necessarily be accelerated as $a$, the axis, is diminished.

And here in passing, we also observe how Kepler's law of the sesquiplicate ratio may be anew proved, but only if we make $\mu=\mathrm{S}$ (the sun), and neglect the mass of the planet. For take two planets whose mean motions are $n$ and $n^{\prime}$ round a third body, and their mean motions being as $\frac{t \sqrt{ } \mu}{a^{\frac{3}{2}}}$ and $\frac{t^{\prime} \sqrt{\mu^{\prime}}}{a^{\frac{13}{2}}}$, and because $\left(2 \pi\right.$ being $\left.360^{\circ}\right)$, $n t=2 \pi$, therefore $t=\frac{2 \pi}{n}$, and $t^{\prime}=\frac{2 \pi}{n^{\top}}$, or $t=$ $\frac{2 \pi \cdot a^{\frac{3}{2}}}{\sqrt{\mu}}$, and $t^{\prime}=\frac{2 \pi a^{\frac{3}{2}}}{\sqrt{\mu^{\prime}}}$; consequently $t^{2}: t^{\prime 2}:: a^{3}: a^{13}$ - being Kepler's law, which is thus demonstrated. But it is only demonstrated and is only true if $\sqrt{\mu}$ is the same to both planets, that is, if $\mu=\mathrm{S}$ in each case. Now, this may be assumed in the case of those bodies revolving round the sun, or of the satellites of Jupiter and Saturn revolving round those primary planets, because of the great disproportion between the central body and the others, (the largest of them, Jupiter, being less than a thousandth part of the sun.) But the law would not hold true if $\mu$ were taken, which in strictness it ought to bc, as $\mathrm{S}+\mathrm{P}$, the sum of the masses of the central and the revolving body; for then $\mu$ would differ in each instance, and the sesquiplicate pro- 
portion would be destroyed. Hence, we arrive through the calculus at this important conclusion, that the law only holds, if the mutual actions of the planets on each other are neglected, and that, therefore, the law is not rigorously true where, as in the case of the earth and others, the actions of the other planets are sensible.

Again, the inspection of the algebraical expressions shows that the variation in the eccentricity of the earth's orbit produces, likewise, the retardation of the apsides and nodes; and this discovery was also made, apparently, by the mere inspection of the expressions which the calculus had furnished. Thus the expression for the motion of the perigee (or apsides) involves the integral $f e^{\prime 2} d v$ ( $v$ being the true anomaly); * and this quantity is positive. Therefore the decrease of the eccentricity of the earth's orbit, causes a decrease, also, of the perigeal motion of the moon. And one of the terms of the equation to the motion of the nodes contains the same integral $f e^{\prime 2} d v$; consequently the same eccentricity is likewise the cause of the variation in the period of their revolution. $\dagger$

Now we have seen how extremely small these irregularities in the moon's motion are which the theory gives by this analytical process, and that they are hardly sensible in a whole century; yet it is found that the deductions of the calculus are in a remarkable manner confirmed by actual observation. Practical astronomers, for example, wholly ignorant of Laplace's discoveries, have ascertained that the secular variation in the motion of the moon's apsides, ascertained by comparing the eclipses in the Greek, Arabian, and Chaldean astronomy, with those of the last

* Angle of the radius vector with the axis of the orbit.

† Méc. Cél. liv. vii. ch. 1. This wonderful chapter is a mere series of integrations, and contains, from the inspection of the equations, those extraordinary discoveries respecting the laws of the universe. 
century, is about 3.3 , or 33 tenths of the moon's mean motion; and this is the exact result of the calculus. Laplace also discovered, chiefly by similar means, a very small secular inequality in the moon's motion never before suspected, and produced by the sun's attraction.* It was found by observing, that the divisor of some of the fractional terms of the equation which shows the inequality is extremely small, and that, consequently, the irregularity may become sensible. A correction of the tables was thus introduced by this great geometrician, in which the theory approaches, on an average, to within $\frac{1}{2} \frac{1}{0}$ of the actual observation. The sign of this inequality being negative, it is a retardation of the mean motion, and is to be set against the secular acceleration. It must be observed, moreover, that the errors of the theory, as compared with the observation, are half of them by excess and half by defect; so that they may be said to balance each other. The maximum of this inequality is little more than $15^{\prime \prime}$, and its period is 184 years.

Hitherto of the moon; but we are, in like manner, conducted by the same refined, though complicated, analysis to the variations in the orbits, and consequently in the motions of the earth and of the other planets, as well as of the satellites of Jupiter and of Saturn. The most remarkable variations produced upon these orbits are the changes in their eccentricity and in their aphelion; the former being constantly, though slowly, shortened - the latter moving round in slow revolutions, as the line of the moon's apsides revolves, but revolves much more swiftly.

The expressions obtained in the case of any one planet for the eccentricity and perihelion longitude (revolving motion of the axis), are mainly composed of the masses, 
distances, eccentricities, and perihelion longitude of the disturbing bodies, with the known eccentricity and longitude of the planet in question at a given epoch. Hence we perceive that on these circumstances depends the varintion of the eccentricity and the revolution of the axis of the planet. Thus the secular variation of the eccentricity of the earth's orbit is 0.000045572 of $e$, the eccentricity which at the epoch (1750) was 0.016814 of the semi-axis major of its orbit; and it has the negative sine in the expression; consequently the eccentricity is on the decrease, as we before observed. This diminution of the eccentricity amounts to about $18^{\prime \prime} 79^{\prime \prime \prime}$ yearly (or about 3900 miles). We have already observed that the annual revolving motion of the axis of the earth's orbit is $11^{\prime \prime} 53^{\prime \prime \prime}$, and its period 109,060 years. The examination of the expressions for these irregularities shows, as might be expected, that Mars, Venus, and Jupiter bear the most considerable share in producing the variations.* But it is a truly remarkable circumstance that the direct action of those planets upon the moon's motion is hardly sensible compared with their indirect, or, as it is sometimes called, reflected action upon the same body, through the medium of the sun and the earth. For these planets, Mars, Venus, and Jupiter, by altering the eccentricity of the earth's orbit, very sensibly affect the motions of the moon, as we have seen, while directly their action is incomparably less perceptible.

The perihelion longitudes of all the other planets are increasing, or their orbits advancing, except Venus, whose apsides are retrograde; and the eccentricities of Venus, Saturn, and Uranus, are decreasing, like that of the earth, whilst those of the other planets are on the 
increase. These variations are greater in Saturn than in any of the others,-considerably greater than the variations of Mars, which comes the nearest to them. The variation in the eccentricity of Jupiter's orbit is nearly three times as great as in the Earth's; that of Saturn between five and six times greater than the Earth's; while the variation in the perihelion longitude of the former is about five-ninths of the Earth's variation; and Saturn's exceeds the Earth's in the ratio of about 25 to 18, and exceeds that of Mars only somewhat more than as 49 to 48 .

When the attention of mathematicians and astronomers was first directed closely to examine the disturbances of these planets, it appeared hardly possible to reconcile such vast and numerous irregularities, as were found to exist, with the theory of gravitation, or indeed to reduce them under any fixed rule whatever. The case seemed to become the more hopeless when so consummate an analyst as Euler, the great improver of the calculus, failed in repeated attempts at investigating the subject, committing several important errors which for a time were not detected, but which showed, or seemed to show, a wide discrepancy between the theory and the observations. By one discovery, indeed, to which his researches led him, he may be said to have laid the foundation of the most extraordinary step which has been made in the knowledge of the planetary system. We allude to his theorem on the periodicity of the eccentricities and aphelia of Jupiter and Saturn. But in most other respects his attempts signally failed. D'Alembert made little progress in this inquiry; but at length Lagrange, and still more Laplace, by applying all the resources of the calculus, in its last stage of improvement, and after the method of Variations had been systematised, succeeded in reducing the whole to order, 
and discovered, while investigating these motions, the great law of the stability of the universe.

The circumstance which mainly contributes to render the irregularities in the motions of two planets great, and which especially augments the disturbance of Jupiter's satellites, is that their mean motions should be commensurable, which those of Jupiter and Saturn are after a very remarkable manner. Five times the mean motion of Saturn are equal to nearly twice that of Jupiter; and the three first satellites of Jupiter are so related to each other, that the mean motion of the first, added to twice the mean motion of the third, is equal to three times that of the second; while the longitude of the first added to twice that of the third, and subtracted from three times that of the second, makes up exactly $180^{\circ}$. Laplace showed, that this proportion, if it was not originally fixed between those satellites, must have been established by the action of the attractive and disturbing forces*; and it is a truly remarkable thing, that when the theory had given a value for the three mean motions, $\mathrm{M}-3 m+2 \mu=0$, the comparison of the eclipses for a century was found to make the expression only $9^{\prime \prime}$, and consequently to tally with the theory within that very small difference. The observation of the effects which were produced upon the equations which resulted from the analysis, by the proportions above stated between the mean motions of Jupiter and Saturn, induced Laplace to suspect that this made quantities become of importance, which from the high powers of the denominators might otherwise have been insignificant. For one of the terms in the equation to $\delta r$ (variation of the radius vector of the first satellite), for example, had for its deno-

* Méc. Cél. lir. vi. ch. 1. 2. 12. 13.; also for the analytical investigation, see liv. viii. throughout, and liv. ii. ch. S. s. 65. 
minator $4\left(n^{\prime}-n\right)^{2} \mathrm{~N}^{2}$ in which $n$ and $n^{\prime}$ are the mean motions of the first and second satellite, and $\mathrm{N}$ a composite quantity not materially differing from $n^{\prime}$, which differs hardly at all from $\frac{n}{2}$, inasmuch as $n=2 n^{\prime}$, while $n^{\prime}=$ $2 n^{\prime \prime}$ ( $n^{\prime \prime}$ being the mean motion of the third satellite); and hence the above denominator becoming little or nothing, the term is of large amount; and so of $\delta v$, the variation of the anomaly. ${ }^{*} \mathrm{He}$ accordingly undertook the laborious task of examining this complicated subject by considering all these quantities; and he arrived at the discovery of, among other inequalities, a retardation of Saturn's motion of about $3^{\prime \prime} 6^{\prime \prime \prime}$ yearly, and an acceleration in Jupiter's motion of about $1^{\prime \prime} 18^{\prime \prime \prime}$. Another irregularity in Saturn's motion with respect to the vernal and autumnal equinox had been observed by astronomers in the last century, and could not be explained. Laplace found this, like all the rest, to follow from the Newtonian theory. In short, when summing up the subject in one of his concluding books, he naturally and justly exclaims, "Tel a été le sort de cette brillante découverte, que chaque difficulté qui s'est élevée, est devenue pour elle un nouveau sujet de triomphe; ce qui est le vrai caractère du vrai système de la nature." $\dagger$

There is no sensible disturbance produced by any of the satellites, except the moon, upon the motion of their primaries, from the extreme smallness of their masses compared with those of the sun and of their primaries; for $\delta r$ is equal to a series in which $\frac{m}{\mathbf{M}}, \frac{m^{\mathrm{i}}}{\mathbf{M}}, \frac{m^{\mathrm{iii}}}{\mathrm{M}}$, \&c., are factors of each term $\ddagger, m, m^{\prime}, \&$ c.,

* Méc. Cél. liv. viii. ch. 1. 4.

$\dagger$ Ibid. liv. xv. ch. 1.-Syst. du Monde, liv. v, ch. 3.

‡ Ibid. liv. vi. ch. 4. 
being the masses of the satellites, and $M$ that of the planets. Now, in the case of Jupiter $\frac{m}{\mathrm{M}}=\frac{1}{57710} ; \frac{m^{\mathrm{ii}}}{\mathrm{M}}$ and $\frac{m^{\text {iv }}}{\mathbf{M}}$ are somewhat greater; but the greatest of the four factors $\frac{m^{\mathrm{iii}}}{\mathrm{M}}=\frac{1}{11302}$ only. But in the case of the earth this factor amounts to about $\frac{1}{80}$; so that $\delta r$ and $\delta v$ become sensible; and will be so, even if, instead of $\frac{m}{\mathrm{M}}$, we take the factor $\frac{m}{\mathrm{M}+m}$, which is more correct.*

When Laplace began his celebrated investigations of the orbits of Jupiter and Saturn, he found that, on substituting numerical values for the quantities in the expression of the mean movement of the one body as influenced by the action of the other, the sums destroyed one another, and left the whole effect of this disturbing force equal to nothing, or the mean motion of neither planet at all affected by the other. The formulas could be in each case reduced to terms only involving two co-efficients; and these destroyed one another. $\dagger \mathrm{He}$ soon found that the same principle applies to all the heavenly bodies; that their mean motions and mean distances (the great axis of their orbits) are not affected by any changes other than those which occur within limited periods of time; that consequently the length of the solar year is precisely the same at any one period of time, as it was at a period so far distant as to enable the changes which are produced within those moderate limits to be effected. This important proposition he demonstrated upon the supposition, 
that the squares of the masses, and the fourth powers of the eccentricities, and the angles of the orbits, are neglected in the calculus.* But Lagrange afterwards showed, that the theorem holds true, even if these quantities be taken into the account. The examination of the moon's motion demonstrates the same important fact, with respect to the permanency of the greater axis and mean motion of the planets; for if the solar day were now $\frac{1}{30} 0$ of a second longer than it was in the age of Hipparchus, the moon's secular equation would be augmented above 42 per cent. or would be in that large proportion greater than it now is known to be. Therefore there has not even been the smallest change of the mean movement of the planets.

The other changes which take place in the orbits and motions of the heavenly bodies, were found by these great geometricians to follow a law of periodicity which secures the eternal stability of the system. The motion of the earth's orbit we have already seen is so slow, that its axis takes above 109,060 years to perform a complete revolution; but after that time it occupies precisely the same position in space as it did when this vast period of time began to run. So the eccentricity of the earth's orbit has been for ages slowly decreasing, and the decrease will go on, or the orbit will approach nearer and nearer to a circle, until it reaches a limit which it never can pass. The eccentricity will then begin slowly to increase until it again reaches its greatest point, beyond which the orbit never can depart from the circular form. The same principle extends itself to all the planets. Thus, the time of the secular variation of Jupiter's eccentricity is 70,400 years.

All these deductions are the strict analytical conse- 
quences of the equations to the eccentricity of the planetary orbits, obtained by the investigation of the total effect of the mutual actions of the heavenly bodies. There results from that analysis this remarkable theorem. That if the eccentricities of the different planets be called $e, e^{\prime}$, $e^{\prime \prime}$, \&c., their masses $m, m^{\prime}, m^{\prime \prime}$, \&c., and their transverse axes $a, a^{\prime}, a^{\prime \prime}, \& c$., and if the integration be made of the differential expression for the relation between the differentials of the eccentricities multiplied by the sines of the longitude and the differentials of the time, and for the relation between the differentials of the eccentricities multiplied by the cosines of the longitudes and the differentials of the time, $\left(\frac{d e \sin . \omega}{d t}\right.$, and $\frac{d e^{\prime} \sin . \omega^{\prime}}{d t, 8 \mathrm{c} .}, \frac{d e \cos . \omega^{\prime}}{d t}$, and $\left.\frac{d e^{\prime} \cos . \omega^{\prime}}{d t}\right)$ \&c., we obtain the equation $e^{2} \cdot m \cdot \sqrt{\bar{a}}+e^{\prime 2}, m^{\prime} \cdot \sqrt{a^{\prime}}+e^{\prime \prime 2}$, $m^{\prime \prime} . \sqrt{ } \overline{a^{\prime \prime}}, \& \mathrm{c} .=\mathrm{C} * \mathrm{C}$ being a constant quantity. Now, as all the motions are in the same direction, $\sqrt{a}, \sqrt{a^{\prime}}, \& \mathrm{c}$., are all positive. Hence, it follows that each of the quantities $e \cdot m . \sqrt{a}, e^{\prime} \cdot m^{\prime} \cdot \sqrt{a^{\prime},} \&$ c., is less than $\mathrm{C}$; and suppose at any one period the whole eccentricities $e, e^{\prime}, e^{\prime \prime}, \&$ c., to be very small, which is known to be true, $\mathrm{C}$, which at that period was the sum of their squares, must be very small, the other quantities $m, m^{\prime}$, \&c., being wholly constant, and $\sqrt{a,} \sqrt{a^{\prime}}$, \&c., being invariable in considerable periods of time. Therefore, it is clear that the variation in any one of those eccentricities, as $e$, never can exceed a very small quantity, namely, a quantity proportional to $\sqrt{\mathrm{C}-e^{\prime 2}-e^{\prime \prime 2}}, \& \mathrm{c}$. The whole possible amount of the eccentricity is confined within very narrow limits. It never can for any body, whose eccentrieity is $e$, excecd a quantity equal to 


$$
\sqrt{\frac{\sqrt{\overline{\mathrm{C}}-e^{\prime 2} \cdot m^{\prime} \cdot \sqrt{ } a^{\prime}-e^{\prime \prime 2} \cdot m^{\prime \prime} \cdot \sqrt{a^{\prime \prime}}-8 \mathrm{c}}}{\sqrt{m \cdot \sqrt{a}}}}
$$

So that the eccentricities never can exceed a very small quantity.

Thus the changes which are constantly taking place in the planetary orbits are confined within narrow limits; and the other changes which are the consequences of this alteration of the orbits, as, for instance, the acceleration of the moon which we before showed arose from the variation of the eccentricity of the earth's orbit, are equally confined within narrow limits. Those changes in the heavenly paths and motions oscillate, as it were, round a given middle point, from which they never depart on either hand, beyond a certain small distance; so that at the end of thousands of years the whole system in each separate case (each body having its own secular periods) returns to the exact position in which it was when these vast successions of ages began to roll. For similar theorems are deduced with respect to other revolutions of the system, whose general destiny is slow and constant change, but according to fixed rules, regulated in its rate, confined in its quantity, limited within bounds, and maintaining during countless ages the stability of the whole universe by appointed and immutable laws.

Laplace examined in the last place the possible effects upon the celestial motions of the resistance of a subtle ethereal medium, and of the transmission of gravity or attraction not being instantaneous, but accomplished in a small period of time. The result of his analysis led him to disbelieve in both these disturbing causes. He found that in order to produce its known effects, the transmission of gravity, if effected in time, must be seven 
millions of times swifter than that of light, or 147 thousand millions of miles in a second.*

iii. The great system of most interesting truths which we have now been contemplating is the work of those who diligently studied the doctrines unfolded by Sir Isaac Newton, respecting the motions of bodies which act upon each other, while they are moving around common centres of attraction. He laid down the principles upon which the investigations were to be conducted; he showed how they must lead to a solution of the questions proposed, touching the operation of disturbing forces; and he exemplified the application of his methods by giving solutions of these questions in certain cases. Although his successors, treading in his steps, have reaped the great rewards of their learning and industry, and are well entitled to all praise for the skill with which they both worked and improved the machinery that he had put into their hands, - at once improving the calculus invented by him, and felicitously applying it to advance and perfect his discoveries, - yet the distance at which his fame leaves theirs is at least equal to that by which a Worcester and a Watt outstripped those who, in later times, have used their mechanism as the means of travelling on land and on water, in a way never foreseen by those great inventors. Strict justice requires that we should never lose sight of the truth repeatedly confessed by Euler, Clairaut, Delambre, Lagrange, Laplace, that all the advances made by them in the use of analysis, and in its application to physical astronomy, are but the consequences of the Newtonian discoveries; so that we are guilty of no exaggeration, if we regard the most brilliant achievements of those great men only as corollaries from the propositions of their illustrious master. Let us briefly see 
how he laid the deep and solid foundations of the fabric which we have been surveying.

After examining the motions of a system of two bodies with respect to one another, and their common centre of gravity, and in space, as those motions are affected by the mutual attractions of the two bodies themselves (in the manner which we have already described), Newton proceeds to the great problem of the Three Bodies, as it has been termed, because the solution is so difficult, that generally the attempt has been confined to the case of three only, this also being sufficient for determining the more important disturbances of the moon's motions. The inquiry, however, is general in the Principia; and its subject is, the motion, produced by the mutual actions upon one another of the bodies in a system. Thus, for example, the inquiry already analysed regards the effect produced upon their motion in space, by the mutual attractions of the earth and moon; that to which we now are proceeding regards their motion, as also influenced by the disturbing force of the sun, and indeed, even by the smaller but not evanescent disturbing forces of the other planets. So as the former inquiry may be extended on the same principles to the motions of Jupiter and Saturn, and their satellites; this new inquiry applies also to the disturbances of their systems by ours, and of our system by theirs.

Newton begins by showing that if the attracting force increases as the distance of the bodies from each other, any two, $\mathrm{M}$ and $\mathrm{E}$, will revolve round their common centre of gravity, $\mathrm{G}$, in an ellipse having $\mathrm{G}$ for its centre. This is plain from what was formerly proved when treating of the conic sections, and also more lately respecting the centre of gravity. If, then, each of these is attracted, in the same manner, by a third body $\mathrm{S}$, this force, being resolved into two, one parallel to the line joining $M$ and 
$\mathrm{E}$, the other parallel to the line joining $\mathrm{E}$ and $\mathrm{G}$, the former force will only accelerate the motion of $M$ and $\mathrm{E}$ round $\mathrm{G}$ by an addition to the mutual attraction of $\mathrm{M}$ and $\mathrm{E}$; the latter force will draw the centre $\mathrm{G}$ towards $\mathrm{S}$ or towards $G^{\prime}$, the common centre of gravity of the three bodies, and combined with the action of $\mathrm{M}$ and $\mathrm{E}$ upon their centre $G$ will make $G$ revolve in an ellipse round $\mathrm{G}^{\prime}$, the common centre of the three, round which also, in like manner, $S$ will describe an ellipse, $G^{\prime}$ being the centre of those two ellipses. Thus the bodies $\mathrm{M}$ and $\mathrm{E}$ will describe an ellipse round the centre $G$, and the centre $G$ and body $S$ will describe ellipses round the centre $G^{\prime}$, both $G$ and $G^{\prime}$ being the centres of these ellipses; and so of any greater number of bodies.-Moreover, the absolute amount of the attractive force in each centre will be as the distance of the centre from the bodies or centres of gravity severally, multiplied by the masses of the bodies. So that $\mathrm{E}$ and $\mathrm{S}$ are attracted to $\mathrm{G}$ by a force as $(\mathrm{M}+\mathrm{E}$ + S) multiplied by their respective distances from G.Lastly, the times in which these ellipses are described by the bodies and the centres, are all equal by what was before proved respecting motion when the force varies as the distances.

This law of the centripetal force is the only one which preserves the entire ellipticity of the orbits, notwithstanding any mutual disturbances; but it produces, at great distances, motions of enormous velocity. Thus we have seen that Saturn would move at the rate of 75,000 miles in a second (or a third of the velocity of light itself), were there no disturbance from the other bodies; but the disturbance might greatly accelerate this rapid motion. If the law be the inverse square of the distance, there will be a departure from the elliptical form of the orbits and from the proportion of the areas to the times, indicating 
that the several resulting forces are not directed towards the several centres. But this departure will be less considerable in proportion as the body in the centre of any system, or in the common centre of any number of systems, is of a magnitude exceeding that of the revolving bodies, or systems of bodies, because this will prevent the central body moving far from its place, or much out of a straight line; and also the departure will be less in proportion as the bodies, or systems revolving, are at a great distance from the centres or from the common centre, because the diminution of this distance increases the inclination of the lines in which the disturbing forces act, and thus disturbs the motions of the bodies among themselves.-Again, if the law of the attraction varies from the inverse square of the distance in some, and not in others, the disturbing effect will be increased. So that we may infer the universality of the law and also the small amount of the disturbing force, and its acting in nearly parallel lines, if we find the ellipticity of the orbits not much deranged, and the proportions of the areas to the times not greatly interrupted.

Newton proceeds to examine more minutely the disturb-

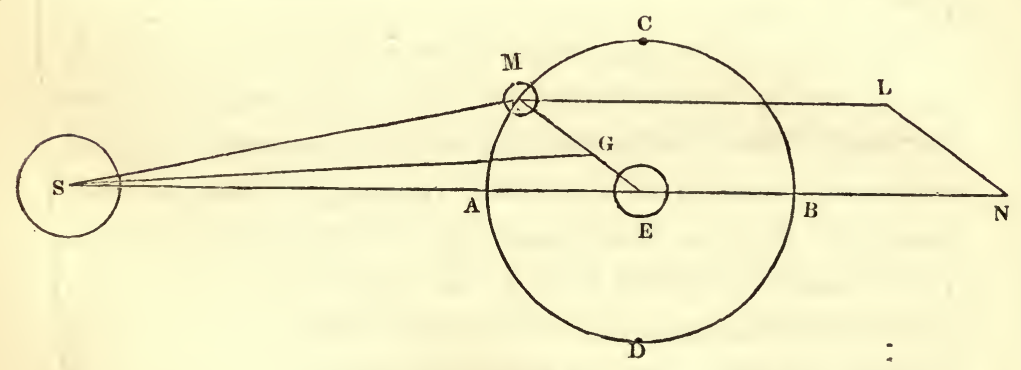

ances caused in a system of Three Bodies, of which two smaller ones move round a third larger one, and all attract one another by forces inversely as the squares of the dis- 
tances. Let $\mathrm{S}$ attract $\mathrm{M}$ with a force inversely as the square of the distance; call the mean distance $=1$; the mean force will be $\frac{1}{1^{2}}=1$. Let the distance from $\mathrm{S}$, successively taken by $\mathrm{M}$ in moving round $\mathrm{E}$, or its true distance, be $\mathrm{SM}$; thence the force at $\mathrm{M}$ is $\frac{1}{\mathrm{~S} \mathrm{M}^{2}}$. Take $\mathrm{SL}=\frac{\mathrm{S}}{\mathrm{S} \mathrm{M}^{2}}$, and drawing $\mathrm{LN}$ parallel to $\mathrm{ME}$, the forces at $M$ are $L N+Q$ ( $Q$ being a quantity that varies as $\frac{1}{M^{2}}$ ) and $\mathrm{SN}$. Now $\mathrm{L} \mathrm{N}: \mathrm{M} \mathrm{E}:$ : $\mathrm{SL}: \mathrm{SM}$; and $\mathrm{L} N=\frac{\mathrm{SL} . \mathrm{ME}}{\mathrm{SM}}=\frac{\mathrm{ME}}{\mathrm{S} \mathrm{M}^{3}} . \quad$ Therefore the force acting upon $\mathrm{M}$ towards $\mathrm{E}$ is as $\mathrm{ME}+\frac{\mathrm{ME}}{\mathrm{SM}^{3}}$; consequently it will increase the attraction of $\mathrm{E}$, but it will not be inversely as the square of the distance; and therefore $\mathrm{M}$ will not describe an ellipse round $\mathrm{E}$, and the force $\mathrm{N}$ S does not tend towards $\mathrm{E}$, nor does the force resulting from compounding $\mathrm{L} \mathrm{N}$, or $\mathrm{ME}$, or $\mathrm{L} \mathrm{N}+\mathrm{M} \mathrm{E}$, with $\mathrm{N} \mathrm{S}$, tend to $\mathrm{E}$. So that the areas will not be proportional to the times. Therefore, also, this deviation from the elliptical form and from the proportional description of the areas will be the greater, as the distances $\mathrm{LN}$ and $\mathrm{N} \mathrm{S}$ are smaller.

Again, let $\mathrm{S}$ attract $\mathrm{E}$ with a force as $\frac{1}{\mathrm{SE}^{2}}$; if this were equal to $\mathrm{S} \mathrm{N}$, it would, by combining with $\mathrm{S} \mathrm{N}$, that is, with the attraction of $\mathrm{S}$ on $\mathrm{M}$, produce no alteration in the relative motion of $\mathrm{M}$ and $\mathrm{E}$. Therefore, that alteration is only caused by the difference between $\mathrm{SN}$ and $\frac{\mathrm{S}}{\mathrm{SE}^{2}}$; wherefore the nearer $\mathrm{SN}$ is to the proportion of $\frac{1}{\mathrm{SE}^{2}}$, that is (because of the 
proportion of $\mathrm{SL}=\frac{1}{\mathrm{SM}^{2}}$ ), the nearer $\mathrm{S} \mathrm{N}$ is to unity, the mean force upon $\mathrm{M}$; and the nearer the forces exerted by $\mathrm{S}$ on $\mathrm{M}$ and on $\mathrm{E}$ approach to equality, the less will the elliptical orbit be disturbed, and the more nearly will the areas be described proportionally to the times. If the disturbing force of $\mathrm{S}$ acts in a plane different from that in which $\mathrm{M}$ and $\mathrm{E}$ are, $\mathrm{M}$ will be deflected from the plane of its orbit; because the force $\mathrm{SN}-\frac{1}{\mathrm{SE}^{2}}$ will not pass through $\mathrm{E}$; consequently this deflection will be greater or less in proportion as this difference is greater or less, and will be least when $\frac{1}{\mathrm{SE}^{2}}$ is nearly equal to the mean force of S upon M.

We have hitherto been supposing $\mathrm{S}$, the greater body round which $\mathrm{M}$ and $\mathrm{E}$ revolve, to be at rest while they revolve round each other (the case of the earth and of other planets having satellites). If we now suppose $\mathrm{E}$ to be the greater and central body, and that $\mathrm{M}$ and $\mathrm{S}$ both move round $\mathrm{E}$ (the case of the planets round the sun), a similar proposition may be demonstrated with respect to the disturbances: And it is further clear in this case that if $\mathrm{S}$ moves round $\mathrm{G}$, the centre of gravity of $\mathrm{M}$ and $\mathrm{E}$, the orbit of $\mathrm{S}$ will be less drawn from the elliptical form, and its radius vector will describe areas more nearly proportional to the times than if it moved round $\mathrm{E}$. This appears clearly from observing that the direction of the centripetal force towards $\mathrm{G}$, that is $\mathrm{S} \mathrm{G}$, must be nearer $\mathrm{E}$ than $\mathrm{M}$; that the attractive forces by which $\mathrm{S}$ is drawn are as $\frac{1}{\mathrm{SM}^{2}}-\frac{1}{\mathrm{SE}^{2}}$; that their resulting force lies in the line $\mathrm{S} \mathrm{G}$; and also that $\mathrm{S} \mathrm{M}$ varies, while $\mathrm{S} \mathrm{E}$ remains the same, or nearly so. 
In all these cases the absolute attractive forces are as the masses of the attracting bodies; and if there are a number of these, A, B, C, E, \&c., of which $\mathrm{A}$ attracts all the rest with forces as $\frac{1}{\mathrm{D}^{2}}, \frac{1}{d^{2}}$, \&cc., (D, $d, \&$ c., being the distances from A,) and $\mathrm{B}$ also attracts A, C, E, \&c., with forces as $\frac{1}{\mathrm{D}^{2}}, \frac{1}{d^{2}}$, the absolute attraction of $A$ and B towards each other are as the masses A and B. Hence in a system, as of a planet and its satellites, if the latter revolve in ellipses, or nearly so, and describe areas proportional, or nearly so, to the times, the forces are mutually as the masses of the bodies; and conversely, if the forces are proportional to the masses, and ellipses are described and the areas as the times, the mutual attractions of all are inversely as the squares of the distances.

It is proved, by reasoning of the same kind, that the disturbing force of $\mathrm{S}$ is greatest when $\mathrm{M}$ is in the points $\mathrm{C}$ and $\mathrm{D}$ of the orbit (or the quadratures), and least when $\mathrm{M}$ is in $\mathrm{A}$ and $\mathrm{B}$ (or the line of conjunction and opposition called the syzygies). When $\mathrm{M}$ is moving from $\mathrm{C}$ to $\mathrm{A}$ and from $\mathrm{D}$ to $\mathrm{B}$, the disturbing force accelerates the motion of $\mathrm{M}$, which then moves along with the disturbing force. When $\mathrm{M}$ moves from $\mathrm{A}$ to $\mathrm{D}$, and from $\mathrm{B}$ to $\mathrm{C}$, its motion is retarded, because the disturbing force acts against the direction of M's motion. So M moves more swiftly in syzygy than in quadrature, and its orbit is more curved in quadrature than in syzygy. But it will recede further from $\mathrm{E}$ in quadrature, unless the eccentricity of the orbit should be such as to counterbalance this recession: for the operation of the combined forces is twofold; it both makes the line of apsides move forward in one point of the body's revolution and backward in another, but more forward than backward, and so upon the whole makes it ad- 
vance somewhat each revolution (as we before saw); and it also increases the eccentricity of the orbit between quadrature and syzygy, and diminishes that eccentricity between syzygy and quadrature. So of the inclination of the orbit, which is always diminished between quadrature and syzygies, and increased between syzygy and quadrature, and is at the minimum when the nodes are in quadrature and the body itself in syzygy.

We found before that the force $\mathrm{L} N$ was as $\frac{\mathrm{M} \mathrm{E}}{\mathrm{SM}^{3}}$. The forces $\mathrm{L} \mathrm{N}$ and $\mathrm{N} \mathrm{E}$ are directly as the mass $\mathrm{S}$, and when $\mathrm{S}$ is very distant, the forces $\mathrm{LN}$ and $\mathrm{N} \mathrm{E}$ vary as $\frac{\mathrm{S}}{\mathrm{SE}^{3}}$, or inversely as the squares of the periodic times; and if at a given distance the absolute disturbing force be as the magnitude of the disturbing body, whose diameter is $d$, these forces are as $\frac{d^{3}}{\mathrm{SE}^{3}}$; or as the cube of the apparent diameter of S. Also if instead of one satellite, M, moving round $\mathrm{E}$, we have several whose orbits are nearly of the same form or inclination (like the first three of Jupiter), the mean motion of their apsides and nodes each revolution are directly as the squares of their periodic times, and inversely as the squares of the planet's time, and the two motions (apsides and nodes) are to one another in a given ratio.

We now have one of those extraordinary instances which abound in his writings, of Sir Isaac Newton's matchless power of generalization; of apprehending remote analogies, and thereby extending the scope of his discoveries. Having shown how the disturbing forces of bodies in a system act upon their motions with respect to each other, he now examines the effect of such forces upon the constitution of the bodies themselves. He supposes, for example, 
that a number of masses of a fluid revolve round $\mathrm{E}$ at equal distances from it by the same laws of attraction by which $\mathrm{M}$ moves round $\mathrm{E}$, and that these masses are thus formed into a ring; then it follows that the portions of this ring will move quicker in syzygy than in quadrature, that is, quicker at $\mathrm{A}$ and $\mathrm{B}$ than at $\mathrm{C}$ and $\mathrm{D}$; also, that the nodes of the ring, or the intersections of its plane with the plane S E, will be at rest in syzygy, and move quickest in quadrature, and that the ring's axis will oscillate as it revolves, and its inclination will vary, returning to its first position, unless so far as the precession of the nodes carries it forward. Suppose now E to be a solid body with a hollow channel on its surface, and that $\mathrm{E}$ increased in diameter until it meets the ring, which now fills that channel, and suppose $\mathrm{E}$ to revolve round its own axis the motion of the fluid, alternately accelerated and retarded (as we have shown), will differ from the equable rotatory motion of the solid on its axis, being quicker than the globe's motion in syzygy, and slower in quadrature. If $\mathrm{S}$ exerts no force, the fluid will not have any ebbs and flows, but move as round a centre that is at rest; but if the varying attraction of $\mathrm{S}$ operates, being greater when the distance is less, the disturbing force acting in the direction $\mathrm{S} L$, and being as $\frac{1}{\mathrm{~S} \mathrm{~N}^{2}}$, will raise the fluid in $\mathrm{A}$ and $\mathrm{B}$, or in syzygy, and from thence to quadrature, C and $\mathrm{D}$, while the force $\mathrm{LN}$ will depress it in quadrature, $\mathrm{C}$ and $\mathrm{D}$, and from thence to syzygy, A and B. If we now suppose the ring to become solid, and the size of $\mathrm{E}$ to be again reduced, the inclination of the ring will vary, and oscillate; and the precession of its nodes will continue the same - and so would the globe, if, without any ring at all, it had an accumulation of matter in the equator, or had matter of greater density there than elsewhere, and 
at the poles. If, on the other hand, there is more matter at the poles, or matter of a less dense kind at the equator, the nodes will advance instead of receding. So that by knowing the motion of the nodes, we can estimate the constitution of the globe; and a perfectly spherical and homogeneous globe will move equally and with a single motion only round its axis. No other will.

The Sixty-sixth Proposition, or rather its twenty-two corollaries, constitute perhaps the most extraordinary portion of the Principia. We have seen that Sir Isaac Newton here deduces most of the leading disturbances in the motions of three bodies, for example, the moon, earth, and sun, from the propositions which had been before demonstrated. We perceive in succession the motion of longitude and latitude; the various annual equations, motion of the apsides (in which, however, by omitting the consideration of the tangential force, he calculated the amount at one half its true value), the evection*, the alteration, and inclination; the motion of the nodes. Even the doctrine of the tides, and the precession of the equinoxes, are all handled clearly, though concisely, in this proposition. The greater part of the Third Book is occupied with the application of these corollaries to the actual case of the moon, earth, and sun; and it is not any exaggeration to affirm that the great investigations which have been undertaken since the time of Sir Isaac Newton, and of which we have just been surveying the principal results, are an application of the improved calculus to continue the inquiries which he thus here began.

The propositions respecting the masses of the attracting bodies which we considered before the corollaries to the

* Laplace has erroneously stated that Newton overlooked the Evection; but it forms, though not by name, the subject of the ninth corollary to this Sixty-sixth Proposition. 
Sixty-sixth Proposition (although they come later in the Principia), and the latter of those corollaries, naturally lead to the subject of the next two sections, the one upon the attraction of spherical bodies, the other upon that of bodies not spherical.

i. The attraction exerted by spherical surfaces and by

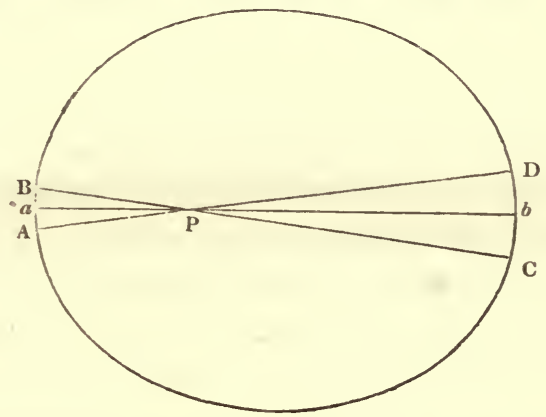

hollow spheres is first considered. If $\mathrm{P}$ be a particle situated anywhere within $\mathrm{ABDC}$, and we conceive two lines A D, B, C, infinitely near each other drawn through $\mathrm{P}$ to the surface, and if these lines revolve round $a \mathrm{P} b$, which passes from the middle points $a$ and $b$, of the small arcs $\mathrm{D} \mathrm{C}$, and $\mathrm{A} \mathrm{B}$, through $\mathrm{P}$, there will two opposite cones be described; and the attraction of the small circles $\mathrm{D} \mathrm{C}, \mathrm{AB}$ upon $\mathrm{P}$, will be in the lines from each point of those circles to $\mathrm{P}$, of which lines $\mathrm{C} \mathrm{P}, \mathrm{DP}$, are two from one circle, and A P, B P, two from the other circle. Now this attraction of the circle $C D$ is to that of the circle $A B$, as the circle $C D$ to the circle $A B$, or as $C^{2}$ to $A$ $\mathrm{B}^{2}$ (the diameters), and by similar triangles $\mathrm{C} \mathrm{D}^{2}: \mathrm{A} B$ $:: \mathrm{P} \mathrm{C}^{2}: \mathrm{P} \mathrm{A}^{2}$. But by hypothesis, the attraction of $\mathrm{C} \mathrm{D}$ is to that of $\mathrm{A} \mathrm{B}$ as $\mathrm{A} \mathrm{P}^{2}: \mathrm{P} \mathrm{C}^{2}$; therefore the attraction of $\mathrm{DC}$ is to the opposite attraction of $\mathrm{AB}$ as $\mathrm{A} \mathrm{P}^{2}$, to $\mathrm{P} \mathrm{C}^{2}$, and also as $\mathrm{P} \mathrm{C}^{2}$ to $\mathrm{A}^{2}$, or as $\mathrm{A}^{2} \times \mathrm{P} \mathrm{C}^{2}$ to $\mathrm{A}^{2}$ $\times \mathrm{P} \mathrm{C}^{2}$, and therefore those attractions are equal; and 
being opposite they destroy one another. In like manner, any particle of the spherical surface on one side of $P$, acting in the direction of $a \mathrm{P}$, is equal as well as opposite to the attraction of another particle acting on the opposite side, and so the whole action of every one particle is destroyed by the opposite action of some other particle; and $P$ is not at all attracted by any part of the spherical surface; or the sum of all the attractions upon $\mathrm{P}$ is equal to nothing. So of a hollow sphere; for every such sphere may be considered as composed of innumerable concentric spherical surfaces, to each of which the foregoing reasoning applies; and consequently to their sum.

We may here stop to observe upon a remarkable inference which may be drawn from this theorem. Suppose that in the centre of any planet, as of the earth, there is a large vacant spherical space, or that the globe is a hollow sphere; if any particle or mass of matter is at any moment of time in any point of this hollow sphere, it must, as far as the globe is concerned, remain for ever at rest there, and suffer no attraction from the globe itself. Then the force of any other heavenly body, as the moon, will attract it, and so will the force of the sun. Suppose these two bodies in opposition, it will be drawn to the side of the sun with a force equal to the difference of their attractions, and this force will vary with the relative position (configuration) of the three bodies; but from the greater attraction of the sun, the particle, or body, will always be on the side of the hollow globe next to the sun. Now the earth's attraction will exert no influence over the internal body, even when in contact with the internal surface of the hollow sphere; for the theorem which we have just demonstrated is quite general, and applies to particles wherever situated within the sphere. Therefore, although the earth moves round its axis, 
the body will always continue moving so as to shift its place every instant and retain its position towards the sun. In like manner, if any quantity of movable particles, thrown off, for example, by the rotatory motion of the earth, are in the hollow, they will not be attracted by the earth, but only towards the sun, and will all accumulate towards the side of the hollow sphere next the sun. So of any fluid, whether water or melted matter in the hollow, provided it do not wholly fill up the space, the whole of it will be accumulated towards the sun. Suppose it only enough to fill half the hollow space; it will all be accumulated on one side, and that side the one next the sun; consequently the axis of rotation will be changed and will not pass through the centre, or even near it, and will constantly be altering its position. Hence we may be assured that there is no such hollow in the globe filled with melted matter, or any hollow at all, inasmuch as there could no hollow exist without such accumulations, in consequence of particles of the internal spherical surface being constantly thrown off by the rotatory motion of the earth.

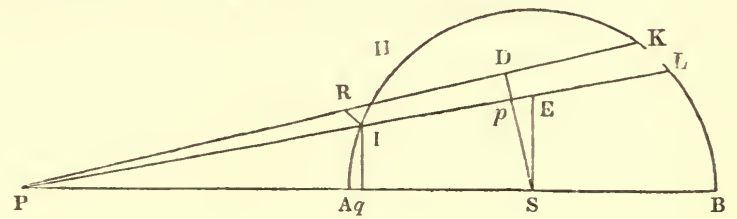

If A H K B be a spherical section (or great circle), PRK and PIL lines from the particle $\mathrm{P}$, and infinitely near each other, SD, SE perpendiculars from the centre, and I $q$ perpendicular to the diameter; then, by the similar triangles $\mathrm{PIR}, \mathrm{P} p \mathrm{D}$, we find that the curve surface bounded by $\mathrm{IH}$, and formed by the revolution of IHKLI round the diameter $A B$, and which is 
proportional to $\mathrm{I} H \times I q$, is as $\frac{\mathrm{I} \mathrm{P}^{2}}{\mathrm{P} p \times \mathrm{PS}}$; and if the attraction upon the particle $\mathrm{P}$ is as the surface directly, and the square of the distance inversely, or $\frac{1}{\mathrm{P}^{2}}$, that attraction will be as $\frac{1}{\mathrm{P} p \times \mathrm{PS}}$. But if the force acting in the line $\mathrm{PI}$ is resolved into one acting in $\mathrm{PS}$ and another acting in $\mathrm{SD}$, the force upon $\mathrm{P}$ will be as $\frac{\mathrm{P} q}{\mathrm{PI}}$, or (because of the similar triangles $\mathrm{PIQ}, \mathrm{PS} p$ ) as $\frac{\mathrm{P} p}{\mathrm{PS}}$. The attraction, therefore, of the infinitely small curvilinear surface formed by the revolution of $\mathrm{I} \mathrm{H}$ is as $\frac{\mathrm{P}}{\mathrm{P} p \times \mathrm{PS}^{2}}$ or as $\frac{1}{\mathrm{PS}^{2}}$; that is inversely as the square of the distance from the centre of the sphere. And the same may be shown of the surface formed by the revolution of $\mathrm{KL}$, and so of every part of the spherical surface. Therefore the whole attraction of the spherical surface will be in the same inverse duplicate ratio.

In like manner, because the attraction of a homogeneous sphere is the attraction of all its particles, and the mass of these is as the cube of the sphere's diameter D, if a particle be placed at a distance from it in any given ratio to the diameter, as $m . \mathrm{D}$, and the attraction be inversely as the square of that distance, it will be directly as $\mathrm{D}^{3}$, and also as $\frac{1}{m^{2} \mathrm{D}^{2}}$, and therefore will be in the simple proportion of $\mathrm{D}$, the diameter. Hence if two similar solids are composed of equally dense matter, and have an attraction inversely as the square of the distance, their attraction on any particle similarly placed with respect to them will be as their diameters. Thus, also, a particle 
placed within a hollow spheroid, or in a solid, produced by the revolution of an ellipsis, will not be attracted at all by the portion of the solid between it and the surface, but will be attracted towards the centre by a force proportioned to its distance from that centre.

It follows from these propositions, first, that any particle placed within a sphere or spheroid, not being affected by the portion of the sphere or spheroid beyond it, and being attracted by the rest of the sphere, or spheroid in the ratio of the diameter, the centripetal force within the solid is directly as the distance from the centre;-secondly, that a homogeneous sphere, being an infinite number of hollow spaces taken together, its attraction upon any particle placed without it is directly as the sphere, and inversely as the square of the distance; - thirdly, that spheres attract one another with forces proportional to their masses directly, and the squares of the distances from their centres inversely; - fourthly, that the attraction is in every case as if the whole mass were placed in the central point; fifthly, that though the spheres be not homogeneous, yet if the density of each varies so that it is the same at equal distances from the centre of each, the spheres will attract one another with forces inversely as the squares of the distances of their centres. The law of attraction, however, of the particles of the spheres being changed from the inverse duplicate ratio of the distances to the simple law of the distances directly, the attractions acting towards the centres will be as the distances, and whether the spheres are homogeneous or vary in density according to any law connecting the force with the distance from the centre, the attraction on a particle without will be the same as if the whole mass were placed in the centre; and the attraction upon a particle within will be the same as if the whole of the body comprised within the spherical 
surface in which the particle is situated were collected in the centre.

From these theorems it follows, that where bodies move round a sphere and on the outside of its surface, what was formerly demonstrated of eccentric motion in conic sections, the focus being the centre of forces, applies to this case of the attraction being in the whole particles of the sphere; and where the bodies move within the spherical surface, what was demonstrated of concentric motion in those curves, or where the centre of the curve is that of the attracting forces, applies to the case of the sphere's centre being that of attraction. For in the former case the centripetal force decreases as the square of the distance increases; and in the latter case that force increases as the distance increases. Thus it is to be observed, that in the two cases of attraction decreasing inversely as the squares of the central distance (the case of gravitation beyond the surface of bodies), and of attraction increasing directly with the central distance (the case of gravitation within the surface), the same law of attraction prevails with respect to the corpuscular action of the spheres as regulates the mutual action of those spheres and their motions in revolution. But this identity of the law of attraction is confined to these two cases.

Having thus laid down the law of attraction for these more remarkable cases, instead of going through others where the operation of attraction is far more complicated, Sir Isaac Newton gives a general method for determining the attraction whatever be the proportions between the force and the distance. This method is marked by all the geometrical elegance of the author's other solutions; and though it depends upon quadratures, it is not liable to the objections in practice which we before found to lie against a similar method applied to the finding of orbits and forces; 
for the results are easily enough obtained, and in convenient forms.

If $\mathrm{A} \mathrm{E} \mathrm{B} \mathrm{is} \mathrm{the} \mathrm{sphere} \mathrm{whose} \mathrm{attraction} \mathrm{upon} \mathrm{the} \mathrm{point}$ $\mathrm{P}$ it is required to determine, whatever be the proportion according to which that attraction varies with the distance, and only supposing equal particles of $\mathrm{A} \mathrm{E} \mathrm{B} \mathrm{to} \mathrm{have} \mathrm{equal}$ attractive forces; then from any point $\mathrm{E}$ describe the circle $\mathrm{E} \mathrm{F}$, and another $e f$ infinitely near, and draw $\mathrm{E} \mathrm{D}, e d$ ordinates to the diameter A B. The sphere is composed of small concentric hollow spheres $\mathrm{E}$ e $f \mathrm{~F}$; and its whole attraction is equal to the sum of their attractions. Now that attraction of $\mathrm{E} e f \mathrm{~F}$ is proportional to its surface multiplied by $\mathrm{F} f$, and the angle $\mathrm{D} \mathrm{E} r$ being equal to D P E (because P E $r$ is a right angle by the property of the circle), therefore $\mathrm{E} r=\frac{\mathrm{PE} \times \mathrm{D} d}{\mathrm{DE}}$, and if we call $\mathrm{P} \mathrm{E}$, or $\mathrm{P} \mathrm{F}=r, \mathrm{E} \mathrm{D}=y$, and $\mathrm{D} \mathrm{F}=x, \mathrm{D} d$ will be $d x$, and $\mathrm{E} r=\frac{r d x}{y}$; and the ring generated by the revolution of $r \mathrm{E}$ is equal to $r \mathrm{E} \times \mathrm{ED}$, or $r \mathrm{E} \times y$; therefore this ring is equal to $r d x$, or the attraction proportional to the whole ring $\mathrm{E} e$ will be proportional to the sum of all the rectangles $\mathrm{PD} \times \mathrm{D} d$, or $(a-x) d x$; that is, to the integral of this quantity, or to $\frac{2 a x-x^{2}}{2}$; which by the property of the circle is equal to $\frac{y^{2}}{2}$. Therefore the attraction of the solid $\mathrm{E}$ ef $\mathrm{F}$ will be as $y^{2} \times \mathrm{F} f$, if the force of a particle $\mathrm{F} f$ on $\mathrm{P}$ be given; if not, it will be as $y^{2} \times \mathrm{F} f \times f$ that force. Now $d x: \mathrm{F} f:: r: \mathrm{P} \mathrm{S}$, and therefore $\mathrm{F} f=$ $\frac{\mathrm{PS} \times d x}{r}$, and the attraction of $\mathrm{E} e f \mathrm{~F}$ is as $\frac{y^{2} \times \mathrm{P} \mathrm{S} \times d x \times f}{r}$; or taking $f=r^{n}$ (as any power of the distance $\mathrm{P} \mathrm{E}$ ), then the attraction of $\mathrm{E} e f \mathrm{~F}$ is as $\mathrm{P} \mathrm{S} \cdot r^{n-1} y^{2} d x$. Take D N 


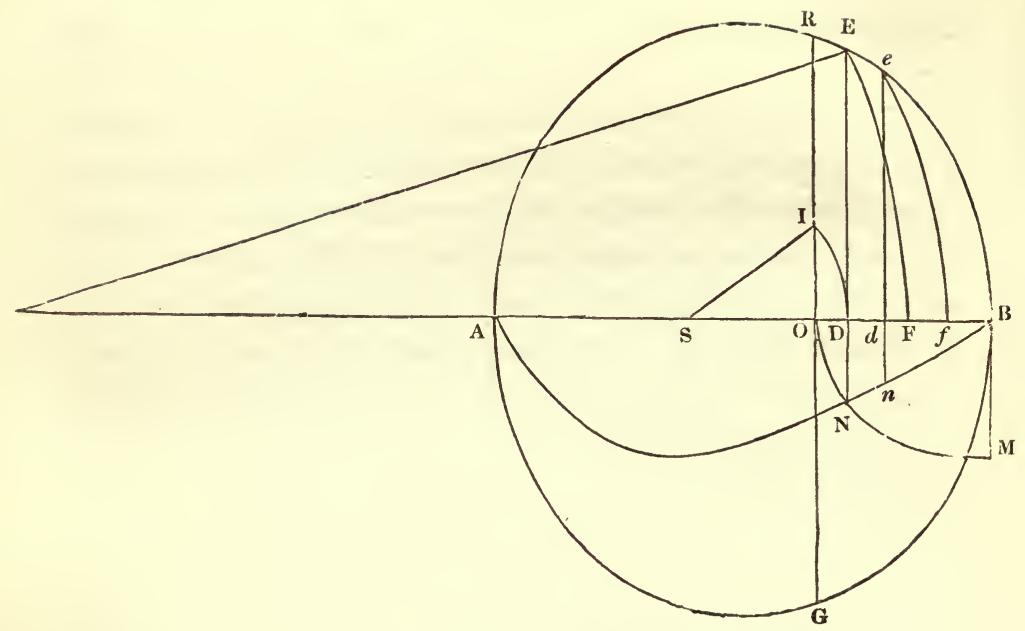

$(=u)$ equal to P S. $r^{n-1} y^{2}$, and let $\mathrm{B} \mathrm{D}=z$, and the curve $\mathrm{BN}$ A will be described, and the differential area $\mathrm{ND} d n$ will be $n d z=$ (by construction) P S. $r^{n-1} y^{2} d x$; consequently $u d z$ will be the attractive force of the differential solid $\mathrm{E} e$ $f \mathrm{~F}$; and $\int u d z$ will be that of the whole body or sphere $\mathrm{A} \mathrm{E} \mathrm{B}$, therefore the area $\mathrm{A} \mathrm{N} \mathrm{B}=f u d z$ is equal to the whole attraction of the sphere.

Having reduced the solution to the quadrature of A N B, Sir Isaac Newton proceeds to show how that area may be found. He confines himself to geometrical methods; and the solution, although extremely elegant, is not by any means so short and compendious as the algebraical process gives. Let us first then find the equation to the curve $\mathrm{AN} \mathrm{B}$ by referring it to the rectangular coordinates $\mathrm{D} \mathrm{N}, \mathrm{A} \mathrm{D}$. Calling these $y$ and $x$ respectively, and making $\mathrm{PA}=b, \mathrm{AS}$ (the sphere's radius) $=a$ and $\mathrm{P} \mathrm{S}$, or $a+b$, for conciseness, $=\frac{f}{2}$. Then $\mathrm{D} \mathrm{E}^{2}=2 a x-x^{2} ; \quad \mathrm{P} \mathrm{E}=\sqrt{(b+x)^{2}+2 a x-x^{2}}$ 
$=\sqrt{b^{2}+2(a+b) x}=\sqrt{b^{2}+f x}$; and $\mathrm{D} \mathrm{N}=y=(\mathrm{by}$ construction) $\frac{(a+b)\left(2 a x-x^{2}\right)}{\left(b^{2}+f x\right)^{\frac{n+1}{2}}}$, the attractive force of the particles being supposed as the $\frac{1}{n}$ th power of the distance, or inversely as $\left(b^{2}+f x\right)^{\frac{n}{2}}$. This equation to the curve makes it always of the order $\frac{n+3}{2}$. If then the force is inversely as the distance, A N B is a conic hyperbola; if inversely as the square, it is a curve of the fifth order; and if directly as the distance, it is a conic parabola; if inversely as the cube, the curve is a cubic hyperbola.

The area may next be determined. For this purpose we have $\int y d x=\int \frac{f\left(2 a x-x^{2}\right) d x}{2\left(b^{2}+f x\right)^{\frac{n+1}{2}}}$. Let $2\left(a f+b^{2}\right)=h$, this integral will be found to be

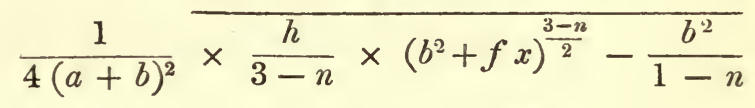

$\times(2 a+b)^{2}\left(b^{2}+f x\right)^{\frac{1-n}{2}}-\frac{\left(b^{2}+f x\right)^{\frac{5-n}{2}}}{5-n}$

$+\mathrm{C}$; and the constant $\mathrm{C}$ is $\frac{1}{4(a+b)^{2}} \times\left(\frac{b^{5-n}}{5-n}\right.$

$\left.+\frac{(2 a+b)^{3} b^{3-n}}{1-n}-\frac{h}{3-n} b^{3-n}\right)$. This in every case gives

an easy and a finite expression, excepting the three cases of $n=-1, n=3$, and $n=5$, in which cases it is to be found by logarithms, or by hyperbolic areas. To find the attraction of the whole sphere, when $x=2 a$, we have $\frac{1}{4(a+b)^{3}} \times\left(\frac{h}{3-n}(2 a+b)^{3-n}-\frac{b^{2}}{1-n} \times\right.$ 
$(2 a+b)^{3-n}-\frac{(2 a+b)^{5-n}}{5-n}+\frac{b^{5-n}}{5-n}+\frac{b^{8-n}}{1-n} \times(2 a+b)^{2}$ $\left.-\frac{h b^{3-n}}{3-n}\right)$ for the whole area $\mathrm{A} \mathrm{N} \mathrm{B,} \mathrm{or} \mathrm{the} \mathrm{whole} \mathrm{attrac-}$ tion. If $\mathrm{P}$ is at the surface, or $\mathrm{A} \mathrm{P}=b=0$, and $n=2$, then the expression becomes as $a$, that is, as the distance from the centre directly. We may also perceive from the form of the expression, that if $n$ is any number greater than 3 , so that $n-3=-m$, the terms $b^{3-n}$ become inverted, and $b$ is in their denominator thus: $\frac{(2 a+b)^{2}}{(1-n) b^{m}}$. Hence, if $n>3$ and $\mathrm{AP}=b=0$, or the particle is in contact with the sphere, the expression involves an infinite quantity, and becomes infinite. The construction of Sir Isaac Newton by hyperbolic areas leads to the same result for the case of $n=3$, being one of those three where the above formula fails. At the origin of the abscissæ we obtain, by that construction, an infinite area; and this law of attraction, where the force decreases in any higher ratio than the square of the distance, is applicable to the contact of all bodies of whatever form, the addition of any other matter to the spherical bodies having manifestly no effect in lessening the attraction.

By similar methods we find the attraction of any portion or segment of a sphere upon a particle placed in the centre, or upon a particle placed in any other part of the axis. Thus in the case of the particle being in the centre $\mathrm{S}$, and the particles of the segment $\mathrm{R} \mathrm{B} \mathrm{G}$ attracting with forces as the $\frac{1}{n}$ power of the distance $\mathrm{SO}$ or $\mathrm{SI}$, the curve A N B will by its area express the attraction of the spherical segment, if $\mathrm{DN}$ or $y$ be taken $=\frac{\mathrm{IO}^{2}}{\mathrm{SD}}$ 
$=\frac{(x-a)^{2}-c^{2}}{(x-a)^{n}}$, S O being put $=c$, and $\mathrm{AD}=x$, and $\mathrm{AS}=a$, as before; $\int y d x$ may be found as before by integrating $\frac{(x-a)^{2} d x-c^{2} d x}{(x-a)^{n}}$. The fluent is $\frac{(x-a)^{3-n}}{3-n}-c^{2} \frac{(x-a)^{1-n}}{1-n}+\mathrm{C} ;$ and $\mathrm{C}=\frac{2 c^{3-n}}{n^{2}-4 n+3} ;$ and the whole attraction of the segment upon the particle at the centre $\mathrm{S}$ is equal to $\frac{a^{3-n}}{3-n}-\frac{c^{2} a^{1-n}}{1-n}+\frac{2 c^{3-n}}{n^{2}-4 n+3}$. Thus, if $n=2$ the attraction is as $\frac{(a-c)^{2}}{a}$, or as $\mathrm{OB}^{2}$ directly, and as S B inversely; and if $c=o$, or the attraction is taken at the centre, it is equal to $a$; and if the attraction is as the distance, or $n=1$, then the attractive force of the segment is $\frac{1}{4}\left(a^{2}-c^{2}\right)^{2}$.

ii. Our author proceeds now to the attractions of bodies not spherical; an inquiry not perhaps, in its greater generality, of so much interest in the science of Physical Astronomy, where the masses which form the subjects of consideration are either spherical, or very nearly spherical, to which our examination has hitherto been confined. But this concluding part, nevertheless, contains some highly important truths available in astronomical science, because it leads, among other things, to determining the attraction of spheroids, the true figures of the planets.

The attractions of two similar bodies upon two similar particles similarly situated with respect to them, if those attractions are as the same power of the distances $\frac{1}{n}$, are to one another as the masses directly, and the $n^{\text {th }}$ power of the distances inversely, or the $n^{\text {th }}$ power of the homo- 
logous sides of the bodies; and because the masses are as the cubes of these sides, $\mathrm{S}$ and $s$, the attractions are as $\mathrm{S}^{3} \cdot s^{n}: s^{3} \cdot \mathrm{S}^{n}$, or as $s^{n-3}: \mathrm{S}^{n-3}$. Therefore, if $n=1$, the attraction is as $\mathrm{S}^{2}: s^{2}$; if the proportion is that of the inverse square of the distance, the attraction is as $\mathrm{S}: s$; if that of the cube, the attraction is as $1: 1$, or equal; if as the biquadrate, the attraction is as $s: \mathrm{S}$; and so on: and thus the law of the attractive force may be ascertained from finding the action of bodies upon particles similarly placed.

Let us now consider the attraction of any body, of what form soever, attracting with force proportioned to the distance towards a particle situated beyond it. Any two of its particles $A B$ attract $P$, with forces as $A \times A P$ and $\mathrm{B} \times \mathrm{BP}$, and if $\mathrm{G}$ is their common centre of gravity, their joint attraction is as $(A+B) \times G P$, because $B P$, being resolved into $B G$ and $G P$, and $A P$ into $A P$ and $G P$, and (by the property of the centre of gravity)

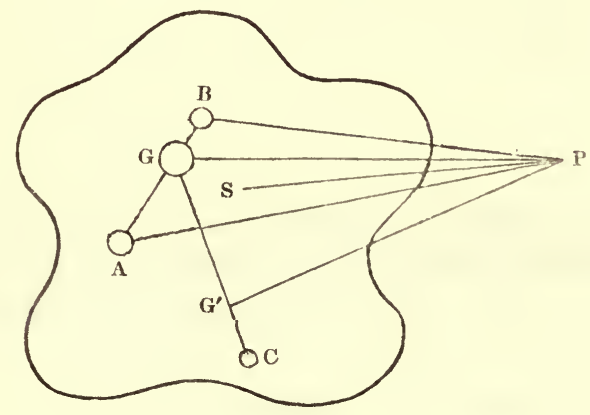

$G P \times A=A P \times G$, therefore the forces in the line $A P$ destroy each other, and there remain only $P G \times B$ and $\mathrm{PG} \times \mathrm{A}$ to draw $\mathrm{P}$, that is $(\mathrm{A}+\mathrm{B}) \times \mathrm{PG}$; and the same may be shown of any other particles $C$ and the 
centre $G^{\prime}$ of gravity, of $A, C$, and $B$, the attraction of the three being $(A+B+C) \times G^{\prime} P$. Therefore the whole body, whatever be its form, attracts $\mathrm{P}$ in the line P S, S being the body's centre of gravity, and with a force proportional to the whole mass of the body multiplied by the distance P S. But as the mutual attractions of spherical bodies, the attraction of whose particles is as their distance from one another, are as the distances between the centres of those bodies, the attraction of the whole body A B C is the same with that of a sphere of equal mass whose centre is in S, the body's centre of gravity. In like manner it may be demonstrated that the attraction of several bodies $\mathrm{A}, \mathrm{B}, \mathrm{C}$, towards any particle $\mathrm{P}$, is directed to their common centre of gravity $\mathrm{S}$, and is equal to that of a sphere placed there, and of a mass equal to the sum of the whole bodies $\mathrm{A}, \mathrm{B}, \mathrm{C}$; and the attracted body will revolve in an ellipse with a force directed towards its centre as if all the attracting bodies were formed into one globe and placed in that centre.

But if we would find the attraction of bodies whose particles act according to any power $n$ of the distance, we must, to simplify the question, suppose these to be symmetrical, that is, formed by the revolution of some plane upon its axis. Let $\mathrm{AM} \mathrm{CHG}$ be the solid, M G the diameter of its extreme circle of revolution next to the particle $\mathrm{P}$; draw $\mathrm{P} \mathrm{M}$ and $p m$ to any part of the circle, and infinitely near each other, and take P D $=\mathrm{P} \mathrm{M}$, and $\mathrm{P} o=\mathrm{P} m ; \mathrm{D} d$ will be equal to $o \mathrm{M}(d n$ being infinitely near $\mathrm{DN}$ ), and the ring formed by the revolution of $\mathrm{M} m$ round $\mathrm{A} \mathrm{B}$ will be as the rectangle $\mathrm{A} \mathrm{M} \times \mathrm{M} m$, or (because of the triangles A P M, $m o \mathrm{M}$, being similar, and $\mathrm{D} d=o \mathrm{M}) \mathrm{PM} \times \mathrm{D} d$, or $\mathrm{PD} \times \mathrm{D} d$. Let $\mathrm{DN}$ be taken $=y=$ force with which any particle attracts at the distance $\mathrm{PD}=\mathrm{PM}=x$, that is as $x^{n}$; and if 
$\mathrm{A} \mathrm{P}=b$, the force of any particle of the ring is as $\frac{b y}{x}$, and the attraction of the ring, described by $\mathbf{M} m$, is as $\frac{b y}{x} \times \mathrm{D} d \times \mathrm{PD}$, or as $b y d x$, and the whole attrac-

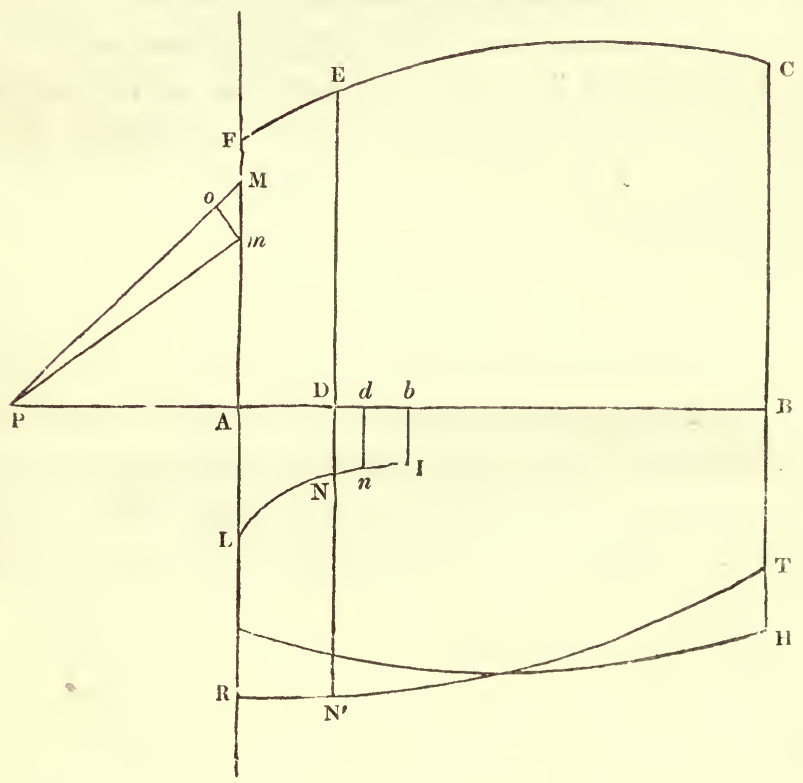

tion of the circle whose radius is $A M$, being the sum of all the rings, will be as $b \int y d x$, or the area of the curve L N I, which is found by substituting for $y$ its value in $x$, that is $x^{n}$. This fluent or area is therefore $=b \int x^{n} d x$ $=\frac{b x^{n+1}}{n+1}+\mathrm{C}$; and $\mathrm{C}=\frac{-b^{n+2}}{n+2} \quad$ Also, making $\mathrm{P}^{n} b$ $=\mathrm{P} \mathrm{E}$ in order to have the whole area of $\mathrm{L} \mathrm{N} \mathrm{I}$, which measures the attraction of the whole circle whose radius is $\mathrm{F} \mathrm{A}$, we have $(x$ being $=\mathrm{P} b=c) \frac{b c^{n+1}}{n+1}-\frac{b^{n+2}}{n+2}$ for 
that attraction. Then taking $\mathrm{D} \mathrm{N}^{\prime}$ in the same proportion to the circle $\mathrm{D} \mathrm{E}$ in which $\mathrm{D} \mathrm{N}$ is to the circle $\mathrm{A} \mathrm{F}$, or as equal to the attraction of the circle $\mathrm{D} \mathrm{E}$, we have the curve $\mathrm{R} \mathrm{N} \mathrm{T}$, whose area is equal to the attraction of the solid L H C F.

To find an equation to this curve, then, and from thence to obtain its area, we must know the law by which $\mathrm{DE}$ increases, that is, the proportion of $\mathrm{DE}$ to $\mathrm{AD}$; in other words, the figure of the section $\mathrm{AFE} \mathrm{C} \mathrm{B,} \mathrm{whose}$ revolution generates the solid.

Thus if the given solid be a spheroid, we find that its attraction for $\mathrm{P}$ is to that of a sphere whose diameter is equal to the spheroid's shorter axis, as $\frac{a \cdot \mathrm{A}^{2}-\mathrm{D} . \mathrm{L}}{d^{2}+\mathrm{A}^{2}-a}$ to $\frac{a^{3}}{3 d^{2}}$, A and $a$ being the two semi-axes of the ellipsoid, $d$ the distance of the particle attracted, and $\mathrm{L}$ a constant conic area which may be found in each case; the force of attraction being supposed inversely as the squares of the distances. But if the particle is within the spheroid, the attraction is as the distance from the centre, according to what we have already seen.

Laplace's general formula for the attraction of a spherical surface, or layer, on a particle situated (as any particle must be) in its axis, is $\frac{2 \pi u d u}{r} \int f d f \times \int d f \mathrm{~F}$, in which $f$ is the distance of the particle from the point where the ring cuts the sphere, $r$ its distance from the centre of the sphere, or the distance of the ring from that centre, $d u$ consequently the thickness of the ring, $\pi$ the semicircle whose radius is unity, and $\mathrm{F}$ the function of $f$ representing the attracting force. The whole attraction of the sphere, therefore, is the integral taken from $f=r-u$ to $f$ 
$=r+u$, and the expression becomes $\frac{2 \pi \cdot u d u}{r}+$ $\int f d f \times \int d f \mathrm{~F}$ with $(r+u)-(r-u)$, substituted for $f$, when $f$ results from this integration. Then let $\mathrm{F}=\frac{1}{f^{2}}$ or the attraction be that of gravitation; the expression becomes $\frac{2 \pi \cdot u d u}{r} \int f d f \times \int \frac{d f}{f^{2}}=\frac{2 \pi \cdot u d u}{r} \times$ $\frac{f^{2}}{2} \times-\frac{1}{f^{\prime}}=\frac{2 \pi \cdot u d u}{r} \times-\frac{(r+u)-(r-u)}{2}=$ $\frac{2 \pi u d u}{r} \times-u=-2 \pi u^{2} d u \times \frac{1}{r}$; and the coefficient of $d r$, taking the differential with $r$ as the variable, is $+\frac{2 \pi u^{2} d u}{r^{2}}$; consequently the attraction is inversely as the square of the distance of the particle from the centre of the sphere, and is the same as if the whole sphere were in the centre.*

The First Book of the Principia concludes with some propositions respecting the motion of infinitely small bodies through media, which attract or repel them in their course, that is to say, of the rays of light, which, according to the Newtonian doctrine, are supposed to be bodies of this kind, hard and elastic, and moving with such rapidity as to pass through the distance of the sun from the earth, or 95 millions of miles, in seven or eight minutes, that is, with a velocity of above 211,000 miles in a second. Sir Isaac Newton shows that, if the medium through which they pass attracts or repels them from the perpendicular uniformly, they describe a parabola, according to Galileo's law of projectiles; but if the attraction or repulsion be

* Méc. Cél. liv. ii. ch. 2. The expression is here developed; but it coincides with the analysis in $\S 11$. 
not equable, another curve will be described; yet, that in either case the sine of the angle of incidence (or that made with the plane where they enter the medium), is to the sine of the angle of refraction (or that made with the plane they emerge from) in a given ratio; that the velocities before incidence and after emerging are inversely as the sines of incidence and refraction; and that if the velocity after incidence is retarded, and the line of incidence inclined more towards the plane of the refracting medium, the small bodies will be reflected back at an angle equal to that of incidence.

$\mathrm{He}$ then remarks on the inflexion and deflexion which light suffers in passing, not through, but by or near bodies, as discovered by Grimaldi *, and as confirmed by his own experiments. He shows that the rays are bent most probably in curve lines, the nearest rays towards the bending body, the furthest rays away from it; and he infers that, in refraction and reflexion, a similar curvilinear bending takes place somewhat before the actual point of refraction and reflexion. He further mentions the colours formed by flexion, as three coloured fringes or bands, "tres colorum fascias." I, however, long ago showed (Phil. Trans. 1797, Part II.) $\dagger$ that this is not the real fact; having found that a much greater number of these fringes are formed by flexion, and that they are, like the prismatic spectrum, images of the luminous body. This experiment has been repeated by Sir David Brewster and others; nor can any doubt be entertained that there are innumerable fringes decreasing in breadth, and in the breadth of the dark intervals between them, until they become evanescent. But as if it were the fate of all this

* Grimaldi termed it diffraction.

† In Phil. Trans., 1850, and Mém. Inst. de France, 1854, are my other papers on Inflexion, showing the same phænomenon, as well as the different flexibility of the rays. 
great man's discoveries, that nothing should ever be added to them but by the use of means which he had himself furnished, it was only by applying a form of experiment which Sir Isaac Newton had used in examining the colours of thick and thin plates, that this important fact was ascertained, he not having subjected the phenomenon first observed by Grimaldi to that mode of investigation.*

The Fourteenth Section concludes with an elegant solution of a local problem in Descartes's Geometry, for finding that form of refracting glasses which will make the rays converge to a given focus, a problem, the demonstration of which Descartes had not given. The brilliant discoveries made by Sir Isaac Newton upon the refrangibility and colours of light, not belonging to dynamics, he pursues the subject no further in this place, having reserved the history of those inquiries for his other great work, the Opticst, perhaps the only monument of human genius that merits a place by the side of the Principia.

The truths which we have been contemplating respecting the attractions of bodies are fruitful in important consequences respecting the constitution of the universe. We have seen that the law of attraction which makes it decrease as the squares of the distances increase, and the law which makes it increase as the distances decrease, are the

* The Undulatory Theory of light, towards which philosophers have of late years appeared to lean, is no exception to this remark; for the principles of that Theory may be found in the Eighth Section of the Second Book of the Principia, and the Scholium which concludes that Section seems to anticipate the application of its principles to Optical Scicnce.

$\dagger$ An abstract of these discoveries had been given in the Lectiones Optice at Cambridge seventeen years before the publication of the Principia in 1687. The Opties only appeared in 1704 . 
only laws which preserve the proportions between the force and the distance, the same for the attraction of the particles of bodies, and for the attraction of the masses in which those particles may be distributed--the only laws which make the attraction of bodies the same with that of their mass placed in the centre of gravity. Now these two laws regulate the actions of bodies gravitating towards each other, the one being the law of gravitation beyond the surface of attracting bodies, the other, the law of gravitation between the surface and the centre. Thus, then, there is every reason to believe that this law pervades the material world universally, acting in precisely the same manner at the smallest and at the greatest distances, alike regulating the action of the smallest particles of matter, and the mightiest masses in which it exists. This action, too, is everywhere mutual; it is always in direct proportion to the masses of the attracted and attracting hodies at equal distances; where the masses are equal, it is inversely as the squares of the distances beyond the bodies, and within the bodies, as the distances from the centre; and where the masses and distances vary, it is as the masses divided by the squares of the distances in the one case, and as the masses multiplied by the distances in the other. This law then pervades and governs the whole system.

The discoveries which astronomers have made since the death of Newton, upon the more remote parts of the universe, by the help of improvements in optical instruments, have further illustrated the general prevalence of the law of gravitation. The double fixed stars, many of which had long been known to astronomers, and which were believed to retain at all times their relative positions, have now been found to vary in their distances from each other, and to move with a velocity sometimes 
accelerated, sometimes retarded, but apparently round one another, or rather round their common centres of gravity. A course of observations continued for above twenty years, led Herschel to this important conclusion about the year 1803; his son has greatly added to our knowledge of these motions; and Professor Struve, of Dorpat, applying geometrical reasoning to the subject, calculated the orbits in which some of the bodies appear to move. One of the most remarkable is the star $\gamma$ Virginis, on which Cassini had made observations in 1720.* It has now been found that one of the stars of which it is composed is smaller than the other; that the revolving motions of the two during the first 25 years had a mean annual velocity of $31^{\prime} 23^{\prime \prime}$; during the next 21 years, of $29^{\prime} 17^{\prime \prime}$; during the next 17 years, of only $2^{\prime} 42^{\prime \prime}$; and during the last two years (1822, $23)$ of no less than $52^{\prime} 51^{\prime \prime}$. The elder Herschel calculated the time of their whole revolution, the periodic times of those distant suns, at 708 years; it is now supposed not to exceed 629. Another pair of stars are found to revolve round one another in between 43 and 44 years, while a third pair take 12 centuries to accomplish their revolution. $\dagger$ Although our observations are far too scanty to lay as yet the ground of a systematic theory of these motions, they appear to warrant us in assuming that the law of attraction which governs our solar system extends to those remote regions, and as their suns revolve round one another, each probably carrying about with it planets that form separate systems, we shall probably one day find that equal areas are there as here described in equal times, and that the orbits are elliptical; or, which would come to the same thing, that the

* Mém. Acad. des Sciences, 1720.

$\dagger$ Phil. Trans. 1803, p. 339; ib. 1824, Part III. 
sesquiplicate proportion of the periodic times and mean distances is observed ${ }^{*}$, from whence the conclusion would of necessity follow, that the centripetal force followed the rule of the inverse square of the distance, and that gravitation such as we know it in our part of the universe, likewise prevails in these barely visible regions. Thus additional confirmation accrues to the first great deduction drawn from the theorems respecting attraction in the Principia.

But other interesting corollaries are also to be deduced from these propositions. They enable us to ascertain, for example, the attractions, the masses, and the figures of the heavenly bodies. Sir Isaac Newton boldly and happily applied them to determine these important particulars, apparently so far removed beyond the reach of the human faculties.

1. The weights of bodies at the surface of the different planets were thus easily determined. The law by which the attractive force of spherical bodies decreases as the square of the distance increases, whether those bodies be homogeneous or not, provided their densities vary in the same proportion, and the other law regulating the proportion between the periodic times and the distances of the planets, enabled him to compute the attraction of each planet, for equal bodies at given distances from their centres, by comparing the observed distances and periodic times of each; and he was thus also enabled, by knowing their diameters, to ascertain the weights of bodies at their surfaces. He found in this manner, that the same body which at the surface of the Earth weighs 435 pounds, at

* It may even seem that already the observed axes of those remote orbits, when compared with their periodic times, approach the sesquiplicate ratio. Thus one his its axis $7 " \cdot 9$, and time 58 years ; and another its axis $30^{\prime \prime} \cdot 8$, and time 452 years. 
that of the Sun weighs 10,000 , at that of Jupiter 943, and at that of Saturn 549.

2. So too the masses of matter in each planet and in the satellites may be ascertained. The motions of the satellites of Jupiter and Saturn afford the easiest means of determining the masses of those planets; and the motions of the other planets round the Sun enable us to solve the problem, though not so accurately, as to them. The mass of Jupiter compared with that of the Earth may be easily supposed to be prodigious, when we find all his satellites revolve round him so much more rapidly than the Moon does round the Earth, although all of them but one have much larger orbits. Thus the second satellite revolves in a seventh of our lunar month, though its path is half as long again: and hence, its velocity is between 10 and 11 times as great. Sir Isaac Newton ascertained the masses of Jupiter, Saturn, and the Earth to be to that of the Sun as $\frac{1}{1067}, \frac{1}{30 \frac{1}{0} T}, \frac{1}{16 \frac{1}{9} \overline{8} \overline{2}}$, to 1 respectively. In like manner the densities are found, being as the weights (first found) divided by the axes. Thus he determined the relative densities of Jupiter, Saturn, and the Earth to be as $94 \frac{1}{2}, 67$, and 400 , to 100 , the density of the Sun. Laplace has ascertained the masses of the heavenly bodies by an entirely different calculus, founded upon the comparison of numerous observations with the formulæ for determining the disturbances. The result is extremely remarkable in one particular. It agrees to a fraction, as regards Jupiter, with the calculation of Newton, making the mass of the planet $\frac{1}{1067}$. But the observations of Pound respecting Saturn's axis, on which Newton had estimated Saturn's mass, were subject to considerable uncertainty; so at least Laplace explains the difference of his own results; but he admits* that

* Méc. Cél. liv. vii. ch. 16, s. 44. 
even in his day there prevailed considerable uncertainty respecting this planet's mass, while that of Jupiter, being well ascertained, agrees perfectly with Sir Isaac Newton's deduction. Laplace gives the masses of the four great planets thus, that of the Sun being unity: Venus $\frac{1}{5 \frac{1}{6} 6 \overline{3}}$; Mars $\frac{2}{2} \frac{1}{4} \frac{1}{6} \overline{5} \overline{0}$; Jupiter $\frac{1}{1067 \cdot 09}$ (differing by $\frac{1}{11}$ only from Newton's, who indeed did ${ }_{a}$ not insert decimals at all); and Saturn $\frac{1}{353^{4} \cdot 08}{ }^{*}$ The Moon's mass he makes $\frac{1}{68.5}$, that of the Earth being unity, while the greatest of Jupiter's satellites is only 0,0000884972 , Jupiter being unity. This great geometrician's observations upon Saturn's ring are peculiarly worthy of attention. The extreme lightness of the matter of which the planet consists, has already been shown; it is six times lighter than the mean density of the Earth; or, if the mean specific gravity of the latter be taken as $5 \dagger$, that of water being as 1, the matter of which Saturn is composed must be only $3 \frac{1}{2}$ times heavier than cork, and lighter than India rubber. But Laplace has satisfactorily shown that his rings must be composed of a fluid, and that no other construction can account for their permanence. $\neq$

3. Sir Isaac Newton, lastly, by the principles which we have been explaining in the latter part of our Analysis, investigated the figures of the heavenly bodies. Thus he especially examined that of the Earth. This planet, in revolving round its axis, gives those particles the greatest tendency to fly off which move with the greatest velocity, that is, those which are furthest from their centres of rotation; in other words, those which are nearest the equator; while those near the poles, describing much smaller circles, move much slower and have far less tendency to

* Méc. Cél. liv. x. ch. 8, 9 ; correcting liv. vi. ch. 6.

$\dagger$ The mean of Maskelyne and Carendish's experiments.

‡ Méc. Cél. liv. iii. ch. 6. 
fly off. Hence there is an accumulation of matter towards the equator, which is raised, while the poles are depressed and flattened, and the equatorial axis is longer than the polar. By comparing the space through which heavy bodies fall in a second in our latitudes with the centrifugal force at the equator, he found that the gravity of bodies there is diminished $\frac{1}{2} \frac{1}{8}$ at least, or that the equatorial axis is, at least, $\frac{1}{2} \frac{1}{89}$ longer than the polar. But he considered this estimate as below the truth, because it does not make allowance for the effect produced on gravitation by the increase of the distance at the equator from the centre. Accordingly, by a skilful application of the method of false position, he corrected this calculation, and ultimately brought out the proportion to be that of 229 to 230 , making the equatorial axis about $34 \frac{1}{5}$ miles longer than the polar, the whole axis being about 7870 miles. $\mathrm{He}$ also estimated the two axes of Jupiter to be as $11 \frac{1}{6}$ to $10 \frac{1}{6}$, supposing the density of the body to be the same throughout; but if it is greater towards the equator, our author observed that the difference between the axes might be decreased as low as 13 to 12, or even 14 to 13 ; which agreed well enough with Cassini's observations in those days, and still more nearly with Pound's. But more accurate observation has since shown that the difference is considerably less, the disproportion being not more than that of 1074 to 1000 ; so that the planet must be very far from homogeneous and its equatorial density greatly exceed its polar. Thus, too, accurate measurements of a degree of latitude in the equatorial and polar regions, and experiments on the force of gravity, as tested by the length of the pendulum vibrating seconds in those different parts of the globe, have led to a similar inference respecting the Earth, its axis being now ascertained to bear the relation, not of 230 to 229 , as 
Newton at last concluded, nor even that of 289 to 288 , according to his first approximation, but only that of 336 to 335 , being an excess of little more than $23 \frac{1}{2}$ miles. The calculation of Newton was formed on the supposition of the Earth being homogeneous; and it is worthy of remark, that although the later observations, by proving the flattening at the poles to be less than he, on this hypothesis, assigned it, have shown the Earth not to be homogeneous, no correction or improvement whatever has been made on his theory in this respect. We find Laplace, on the contrary, in the very passage to which we are now referring, assuming his precise fraction $\frac{1}{2} \overline{0} 0$ as the one given by the theory upon the supposition of the globe being homogeneous, and reasoning upon that fraction. $\dagger$

Now it is fit that we here pause to contemplate perhaps the most wonderful thing in the whole of the Newtonian discoveries. The subject of curvilinear motion, or motion produced by centripetal forces, was certainly in a great measure new, and Sir Isaac Newton's treatment of it was in the highest degree original and successful. But the laws of attraction, the principles which govern the mutual actions of the planets, and generally of the masses of matter, on each other, was still more eminently a field not merely unexplored, but the very existence of which was unknown. Not only did he first discover this field, not only did he invent the calculus by means of which alone it could be explored, and without which hardly a step could be made across any portion of it (for the utmost resources of geometrical skill in the hands of the Simsons and the Stewarts themselves, who in other inquiries had performed such wonders by ancient ana-

* Méc. Cél. liv. iii. ch. 5. $\dagger$ Ibid. liv. iii. ch. 5, s. 41 . 
lysis, would have failed to do anything here), but the great discoverer actually completed the most difficult investigation of this new region, and reached to its most inaccessible heights, with a clearness so absolute, and a certainty so unerring, that all the subsequent researches of his followers, and all their vast improvements on his calculus, have not enabled them to correct by the fraction of a cipher his first results. The Ninetieth and Ninetyfirst Propositions of the First Book, containing the most refined principles of his method, are applied by him in the Nineteenth of the Third Book to the problem of the Earth's figure; his determination of the ellipticity, supposing the mass homogeneous, is obtained from that application. A century of study, of improvement, of discovery has passed away; and we find Laplace, master of all the new resources of the calculus, and occupying the heights to which the labours of Euler, Clairaut, D'Alembert, and Lagrange have enabled us to ascend, adopting the Newtonian fraction of $\frac{1}{2} \frac{1}{5}$, as the accurate solution of this speculative problem. New admeasurements have been undertaken upon a rast scale, patronized by the munificence of rival governments; new experiments have been performed with improved apparatus of exquisite delicacy; new observations have been accumulated, with glasses far excecding any powers possessed by the resources of optics in the days of him to whom the science of optics, as well as dynamics, owes its origin; the theory and the fact have thus been compared and reconciled together in more perfect harmony; but that theory has remained unimproved, and the great principle of gravitation, with its most sublime results, now stands in the attitude, and of the dimensions, and with the symmetry, which both the law and its application received at once from the mighty hand of its immortal author. 


\section{NOTE.}

The argument in page 136. is succinctly and popularly stated respecting the supposition of a hollow in the centre of the Earth, and several steps are omitted. One of these may be here mentioned in case it should appear to have been overlooked. Suppose a mass $m$ detached from the hollow sphere M, and impelled at the same time with that sphere by an initial projectile force,then its tendency would be to describe an elliptic orbit round the sun, the centre of forces, and if it were detached from the earth it would describe an ellipse, and be a small planet. But as the accelerating force acting upon it would be different from that acting on the earth, the one being as $\frac{\mathrm{S}+\mathrm{M}}{\mathrm{D}^{2}}$, and the other as $\frac{\mathrm{S}+m}{\mathrm{D}^{2}}$ (D being the distance and $\mathrm{S}$ the mass of the sun), it is manifest that, sooner or later, its motion being slower than that of the hollow sphere, if $m$ be placed in the inside, it must come in contact with the interior circumference of the sphere, and either librate, or, if fluid, coincide with it, as assumed in the text. Where parts of the spherical shell come off by the centrifugal force, of course no such step in the reasoning is wanted; nor is it necessary to add that neither those parts nor any other within the hollow shell can have any rotatory motion. 


\section{II.}

Hitherto we have considered all motion as performed in vacuo, or in a medium which offers no resistance to the action of forces upon bodies moving in any direction. It was necessary that the subject should first be discussed upon this supposition; and the hypothesis agrees with the fact as far as the motions of the heavenly bodies are concerned. But all the motion of which we have any experience upon or near the surface of the earth, is performed in the atmosphere that surrounds our globe; and therefore, as regards all such motion, a material allowance must be made for the resistance of the air when we apply to practice our deductions from the theory. It is also obvious that a still greater effect will be produced upon moving bodies, if their motion is performed in a denser fluid, as water. Further, the pressure and motion of fluids themselves form important subjects of consideration, independent of any motion of bodies through them and impeded by them. These several matters form the subject of the sciences of Hydrostatics, Hydraulics, and Pneumatics; the first treating of the weight and pressure of watery fluids, the second of their motion, the third of aeriform or elastic fluids. They are discussed in the Second Book of the Principia. It consists of Nine Sections; of which the First Three treat of the motion of bodies to which there is a resistance in different proportions to the velocity of the motion; the Fourth treats of circular or rather spiral motion in resistirg media; the Sixth, of the motion and resistance of pendulums; and part of the Seventh discusses the motion of projectiles; while the rest of the Seventh, and the whole of the four 
remaining sections, treat of the pressure and motion of fluids themselves and propagated in pulses, or otherwise, through fluids. We shall arrange the subjects under these Five heads, instead of following the precise order of the work itself.*

Two observations are applicable to this branch of the subject, and to the treatment of it in the Principia; and these observations lead to our distinguishing this portion of that great work from the rest.

First. Much more had been accomplished of discovery respecting the dynamics of fluids before the time of Sir Isaac Newton, in proportion to the whole body of the science, than in the other branches of Mechanics. The Newtonian discoveries, therefore, effected a less considerable change upon this department of Physics than upon Physical Astronomy and the general laws of motion. As early as the time of Archimedes the fundamental principle of the general or undequâque pressure of fluids had been ascertained; many of the easier problems, and even some of the more complicated, had been investigated by its aid. When dynamical science was newly constructed by the illustrious Galileo, the progress which he made may almost be said to have formed Hydrostatics and Hydraulics into a system; and Pascal's original and inventive genius, socn afterwards applied to it, enabled him clearly to perceive the hydrostatic paradox, and even led him to a plain anticipation of the hydrostatic press. $\dagger$ Torricelli about the same period reduced the atmosphere under the power of weight and measure, making it the subject of calculation by the beautiful experiment which first ascertained its gravity,

* For the arrangement, sce the Summary of Contents.

† He calls a box of water "a new mechanical principle by which we may multiply force ad libitum." (Equil. of Fluids, 1653.) 
which had long been suspected but not proved. Pascal first extended the Torricellian experiment to all the perfection, indeed, which it has ever attained, by showing the connexion between the height of places on the earth's surface, and that of the mercurial column; thus demonstrating satisfactorily the pressure of the atmospherical column. Torricelli had also, from experiments on the spouting of water, inferred that the velocity of the spouting column, or jet, is as the square root of the height of the reservoir of fluid whose pressure causes the flow. So that the fundamental principles being ascertained, considerable progress was also made in their systematic application, when Sir Isaac Newton came to treat the subject as a branch of his general dynamical theory, and to investigate the laws of fluids by means of those profound principles which he had established with respect to all motion. Thus more was done before his time, and less consequently left for him to do here, than in the other branches of the general subject.

Secondly. It is also true that the work which he produced upon this branch of science, did not attain the same perfection under his hands, as the rest of the Principia. Although he treated it upon mathematical principles, he left considerably more to be done by his successors than he left to be added by those who should follow him in the field of Physical Astronomy. A great step was almost immediately made by J. Bernouilli, in ascertaining the effects of the air's resistance upon the motion of projectiles; and an error so apparent was pointed out in one of the Propositions in the Principia (Book II. Prop. 37*), that the correction coming to the author's knowledge, he struck it out of the second edition, then in the press. His original solution of the problem as to spouting columns,

* First Edition, published in 1687. 
having differed from the rule which Torricelli had deduced experimentally, Newton again investigated the question by a different and an admirable process; but eren now the subject remains in a very unsatisfactory state. Nor can it be said that the science of hydrodynamics generally has attained the perfection of the other branches of Mechanical philosophy; while it is certain that the application to it of the calculus by Euler and D'Alembert*, and still more by Clairaut, has greatly added to the theorems left by Sir Isaac Newton; and the researches of Laplace upon capillary attraction form a department of science almost unknown before the latter part of the eighteenth century.

The statement of these particulars was necessary in order to place the relative merits of the different branches of the Principia in their true light. That a great improvement was accomplished in natural knowledge by this portion of Sir Isaac Newton's discoveries, none can doubt. That the Second Book displays at every step the profound sagacity and matchless skill of its author, is undeniable. That it would have conferred lasting renown upon any one but himself, had it been the only work of another man, is cerlain. Nor can we forget that in rating its importance as we have ventured to do, we only undervalue this portion of the Principia, by applying to it the severest of standards, comparing it with the discovery of the laws which govern the system of the universe, and placing it in contrast with the other parts of that unrivalled effort of human genius.

* Their invention of the Calculus of Partial Differences was connected with this subject. (See Life of D'Alembert.) 



\section{ANALYTICAL VIEW.}

\section{- PRINCIPIA.-BOOK SECOND.}

\section{CHAPTER I.}

THE Elementary PRINCIPles OF hydrostatics, AND THE LAWS OF DENSITY OF AN ELASTIC FLUID COLLECTED ROUND A CENTRE OF FORCE. -

I. Elementary Principles of Hydrostatics.

1. What a fluid is, the terms viscosity, solidity, \&c.

2. What the foundation is on which the theory of Hydrostatics is built. Newton, $\mathbf{x I x}$.

3. The fundamental equation by which we know the properties of a fluid in equilibrium. Note $\mathrm{I}$.

4. Three consequences of this equation. Note I.

(1.) That there must in all cases be a certain relation among the forces.

(2.) Level surfaces are surfaces of equal density.

(3.) Level surfaces are surfaces of equal temperature.

5. Newton, xx., Fliuds under the action of gravity only.

II. The Law of Density in a compressible Fluid under the Action of a central Force.

1. Solution of the question - defect in the application to physical questions, Newton, xxi. xxI. \&c.

2. First application. Measurements of heights. Note II.

Second application. Form of our atmosphere, the Zodiacal light not part of the Sun's atmosphere. Note II.

I. 1. "Hydrostatics" is that part of statics which treats of the equilibrium of fluids. A fluid is any bodly whose prarts 
yield to any force impressed on it, and by yielding are easily moved among themselves. This is Newton's definition. It includes gases and aeriform bodies, as well as those to which we, in ordinary conversation, apply the terms "fluid" or " liquid."

The fundamental idea of a fluid is, that of a body whose particles may be moved amongst each other on the application of the slightest possible force. It is therefore directly opposed to a rigid body, whose definition is that its particles cannot be moved amongst each other, no matter how great a force is applied. It is evident that no substance that we meet with in nature is strictly either a fluid or a rigid body; but they approach more or less to the one or the other. When they partake more of the fluid than the rigid nature, they are called "viscous;" when the contrary, they are called "solid." These two are therefore indefinite terms, and no clear boundary can be drawn between them.

2. The science of Hydrostatics is divided into two parts. In one we assume certain general principles as the grounds of all our reasoning. We may consider these as established either by experiment, or as truths which it is the office of the other part of the science to demonstrate. In the other we make certain general assumptions as to the constitution of a fluid, and then we attempt to deduce from these the general principles on which all the rest of hydrostatics is founded. This division occurs in most mechanical sciences. Thus, in Geometrical Optics, we assume the laws of reflection and refraction; it is the part of Physical Optics to establish their truth. It is not here our office to enter into the science of Molecular Hydrostatics; we must postpone, therefore, such consideration to a future chapter.

The mathematical theory of Hydrostatics is founded upon two laws. 
1. The pressure of the fluid upon any element of a surface exposed to it is normal to that surface.

2. Any pressure communicated to a fluid mass in cquilibrium is equally transmitted through the whole fluid in every direction.

Consider any point in a fluid, and let an indefinitely small plane pass through it; by the second law the pressure is the same, whatever be the inclination of the plane to the horizon; by the first it is normal, and proportional to the area of the plane. Let this area be $\alpha$; then the pressure may be represented by $p a$. This quantity $p$ is therefore what we seek to find. It is what the pressure would be if the area were unity, and the pressure constant over that area. It is therefore called the "pressure referred to a unit of area."

The law expressing the equality of pressures in all directions is true in viscous as well as perfect fluids. The difference is this, that in the latter the transmission of the pressure is effected in a moment, in the former it takes time. During this interval the law is not true; but when a short time has been allowed to pass, the fluid takes up its form of equilibrium, and the pressure becomes equal in all directions.

These two laws are not independent. The first contains the second. For, let the fluid contained within the pyramid $\mathrm{O} \mathrm{ABC}$ in the interior of the fluid become solid. This is allowable, for, the fluid being in equilibrium, the pressure on the solidified element will be borne and resisted in exactly the same

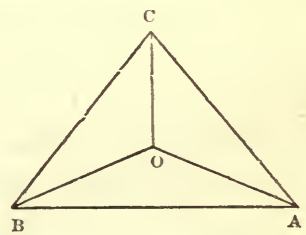

way that it was while still fluid. Let $p$ be the pressure referred to a unit of area on the plane $\mathrm{COA}$ at $\mathrm{O}, q$ that on a plane parallel to $\mathrm{BCA}$ through $\mathrm{O}$. Let the 
pyramid diminish without limit, the pressures on the two sides $\mathrm{COA}$ and $\mathrm{BCA}$ will be normal and respectively equal to

$$
p \times \text { area C OA, } q \times \text { area } \mathrm{BCA} \text {. }
$$

Resolving these, parallel to $\mathrm{O} B$, we have

$$
\frac{p \text { area } \mathrm{COA}}{q \text { area } \mathrm{BC} \mathrm{A}}=\frac{\text { area } \mathrm{COA}}{\text { area } \mathrm{BC} \mathrm{A}},
$$

because this latter ratio expresses the cosine of the inclination of the two planes; hence

$$
p=q \text {. }
$$

Similar equations hold by symmetry for the other sides. And therefore the pressure is equal in all directions. This includes Prop. xIx. of Section V.

$$
\text { 4. }\} \text { Note I. }
$$

5. Newton proceeds to consider the equilibrium of a spherical mass of fluid, like our atmosphere, resting upon a spherical concentric bottom, and gravitating towards the centre of the whole. The object is to determine the pressure on any point $A$ of the bottom. Divide the fluid into concentric orbs of equal thickness $d x$. Now any part of a fluid at rest may be supposed to become rigid; for it will then resist and be resisted by the remainder of the fluid in exactly the same manner as before. Draw, then, any cylindrical canal from the point $\mathrm{A}$ to any point $\mathrm{B}$ in the surface of the fluid, and suppose its superficies to become rigid. This canal will be divided into elements by the concentric orbs. Let $d s$ be the length of any one of these elements, and $F$ the force of gravity; then the weight of that element is $F d s$. This acts directly towards the centre, that is, along $d x$. Resolving along the canal, the force with which this element tends to press the bottom of the canal is $F d x$. The same is true for 
all the elements; hence the whole pressure on the bottom of the canal is

$$
\int F d x
$$

that is to say, it is equal to the weight of a cylinder of fluid, whose base is the area of the part $A$ of the bottom, and whose altitude is the same as that of the superincumbent fluid.

The bottom is not pressed by the whole weight of the incumbent fluid, but only that part which is described above; and it will be the same whether the fluid rises perpendicularly above $A$ in a rectilinear direction, or whether it be contained in crooked cavities and canals, whether these passages be regular or irregular, wide or narrow.

If a body of the same specific gravity as the fluid, and incapable of condensation, be immersed in the fluid, it will neither acquire motion by the pressure of the fluid, nor any change of figure. Any portion of a fluid at rest may clearly be supposed to become solidified without affecting the equilibrium. Let a part of the fluid equal and similar to the body about to be immersed become solid; removing it we may replace it by this body, and the equilibrium will still subsist. It also follows that the resultant of all the pressures exerted by the fluid on the solid is a force equal to the weight of the fluid displaced acting upwards through the centre of gravity of the volume of the body. If, therefore, a solid be immersed in a liquid, it will remain at rest if it be of the same density as the fluid. But if it be of greater density, it will be no longer sustained by the resultant pressures, and will sink to the bottom. If it be of less density, it will rise to the surface, being acted on upwards by a greater force than its own weight. Hence, Newton concludes, bodies placed in fluids have a twofold gravity; one true and absolute, the other apparent, vulgar, 
and comparative. Absolute gravity is the whole force with which the body tends downwards. Relative gravity is the excess of gravity with which the body tends downwards more than the ambient fluid. The bodies, therefore, which we call light, and which appear to fall so slowly, or even seem to rise in the air, are light only in comparison with the air. If there were no air, their apparent gravity would be their real gravity, and all bodies are found to fall when placed in a vacuum.

II. 1. Having discussed some of the fundamental properties of fluids, and obtained the equations of equilibrium, we can proceed to apply them to some of the great problems that Nature presents us with. The first case which Newton considers is the law of density in a compressible fluid which is attracted according to any law by a force tending towards a given centre. $\mathrm{He}$ does not consider this problem in its most general form, nor would there be any advantage in doing so. The only forces which present any interest are those which vary according to some power of the distance.

Let us assume that the attraction upon any particle whose mass is a unit, and distance from the centre $x$, is,

$$
\frac{\mu}{x^{n}}
$$

where $\mu$ is some constant quantity, of $n+1$ dimensions. The fluid will manifestly arrange itself symmetrically round the centre of force. We may therefore consider only those particles that lie in the axis of $x$. Take therefore a small rectangular element at a distance $x$ from the centre of the earth, and whose sides are $d x d y d z$. This element must be at rest under the action of the fluid pressures on its sides and its own gravity. If $p$ be the pressure referred to a unit of area at this point, these two pressures will be clearly 


$$
p d y d z \text { and }-\left(p+\frac{d p}{d x} d x\right) d y d z
$$

acting along the axis of $x$. And the weight will be

$$
-\rho \cdot \frac{\mu}{x^{n}} \cdot d x d y d z ;
$$

and since there is equilibrium the sum of these must be zero. The equation of fluid equilibrium is then

$$
d p=-p \cdot \frac{\mu}{x^{n}} \cdot d x--
$$

To solve the problem we require the relation between $p$ and $\rho$. In fluids generally we have

$$
p=x_{p} \quad-\quad-\quad-(2) \text {. }
$$

This is the law which Newton takes for granted in the two cases which he has worked at length. He also states the results that would be arrived at if we had assumed other laws; and, as we shall see, Laplace has been led to beliere that the above is far from being true within the earth.

Substituting from the second equation the value of $p$ in the first,

$$
x d p=-p \frac{\mu}{x^{n}} d x .
$$

Hence dividing by $\rho$ and integrating,

$$
x \log \rho=\mathrm{C}+\frac{\mu}{n-1 x^{n-1}}-\cdots
$$

where $\mathrm{C}$ is some unknown constant. Hence

$$
p=\mathrm{D} s^{\frac{\mu}{(n-1) x} \cdot \frac{1}{x^{n-1}},}
$$

where $\mathrm{D}$ is the density at the centre or at an infinite distance, according as $u$ is less or greater than unity, and can only be determined by some of the given conditions of the fluid.

Generally, we conclude from the above, that when the 
reciprocals of the $(n-1)^{\text {th }}$ powers of the distances are in arithmetical progression, the densities at those points will be in geometrical progression.

Two cases of the above are worthy of notice, when $n=2$ and when $n=0$. In the former the force attracts inversely as the square of the distance, and the density at any point is given by

$$
\rho=\mathrm{D} \cdot \varepsilon^{+\frac{\mu}{x} \cdot \frac{1}{x}}
$$

that is, if the distances be in harmonical progression, the densities will be in geometrical progression. In the latter case the force is constant and equal to $\mu$, and the density is given by

$$
\rho=\mathrm{D} \cdot \varepsilon^{-\frac{\mu}{k} x}
$$

that is, if the distances decrease in arithmetical progression the densities will decrease in geometrical progression. These cases we might suppose to bear some analogy to the state of our atmosphere, the former holding when the changes of elevation are great, the latter when they are small.

There is one case, especially considered by Newton, in which the preceding general formula fails, viz. when $n=1$, for then $\log \rho$ in equation (3) appears to be always infinite; but this is not really the case, for $\mathrm{C}$ is also infinite and negative. The form of the integral has changed, and by merely repeating the process, we get

$$
\begin{gathered}
x \log \rho=\mathrm{C}-\mu \log x \\
\therefore \rho=\mathrm{D} \cdot x^{-\frac{\mu}{2}}
\end{gathered}
$$

The preceding investigations are not, however, of any very great practical utility. They are all founded on the supposition that the compression varies as the density. 
Now this is only true when the temperature is constant. When it is not, we have seen that the true law is that

$$
p=x p(1+\alpha t) \text {. }
$$

If, however, we attempt to use this equation, we require to know the law according to which $t$, the temperature, varies as we ascend into the air and descend into the earth. We can have but little assistance in determining this from observation. As Humboldt* has remarked, our experimental knowledge of the interior of the earth is limited in the extreme. The greatest depth below the surface of the sea that has yet been obtained, is probably that of the salt-works of New-Salzwerk, near Minden, in Prussia; yet this was only 1993 feet, or less than इ $\frac{1}{20} \overline{0} \overline{0}$ part of earth's radius. The observations even on these small depths are liable to serious errors, - as the different periodic variations of temperature caused by the diurnal or annual heating of the surface, the greater exposure to the surface air, \&c. The temperature of water at the bottom of the salt mine was $90.8^{\circ}$ Fahrenheit, giving a mean decrease of $1^{\circ}$ Fahrenheit for every 53.8 feet. If we tried to make our observations on the law of density instead of that of temperature, - for the knowledge of either would enable us to integrate the equations, - we can succeed no better. The dippings of strata beneath the surface, which rise again at known distances, only reach some twelve thousand feet below the surface of the sea; and if to this we added the height of the highest mountain, we have only a knowledge of $3^{\frac{1}{2}} \frac{1}{4}^{\text {th }}$ part of earth's radius.

We have also observations made on the temperature of the air at the summits of mountains, and in balloon 
ascents. The former will not furnish us with the required law, because the presence of the mountain will affect the temperature of the air by its radiation of the solar rays. Gay Lussac, in his celebrated aerostatic ascent of $3816 \cdot 12$ fathoms, found the temperature at the upper station 14.9 , giving a depression of $1^{\circ}$ for every $95 \cdot 14$ fathoms. A great variety of observations have been made, and many empirical laws invented to suit them. To mention only one: Mr. Atkinson, in the second volume of the Transactions of the Astronomical Society, asserts, that at an altitude of $h$ feet the depression in temperature will be given by

$$
n=\frac{h}{251+\frac{h}{200}}
$$

nearly. We might make use of these results, and by repeating our calculation in the manner indicated, obtain various formula to determine the density at any point. But such results can never be very trustworthy.

II. 2. Note II. 


\section{CHAPTER II.}

\section{THE FIGURE OF THE EARTH.}

1. Newton's calculation of the ellipticity of the earth - its defects.

2. An accurate investigation of the ellipticity on the supposition that the earth is homogeneous - the form thus found proved to be stable. Note III.

3. Newton's calculation of the law of variation of gravity.

4. Newton's application of his theory to the planet Jupiter.

5. The figure of the earth considered as heterogenenus, Clairaut and Laplace's results. Note IV.

a. The form of the strata.

B. The law of variation of gravity.

6. The law of density in the interior of the earth. Note IV.

7. Whether the interior of the earth is solid or fluid. Note IV.

8. Measures to determine by observation the ellipticity of the earth's surface. Note IV.

a. Measurement of degrees.

B. Observations on the pendulum.

$\gamma$. Astronomical observations.

1. Iv the eighteenth proposition of the third book, Newton considers why the earth and planets are protuberant at their equator. He does not investigate the form of the earth, but merely shows that if it had been originally fluid, the matter, by its ascent towards the equator, would enlarge the diameters there, and by its descent towards the poles it will shorten the axis. And even if the earth had not been originally fluid, yet if the earth were not higher at the equator than at the poles, the seas would subside about the poles, and rising towards the equator, would lay all things there under water.

Taking for granted that the true form of the earth is 
a spheroid, Newton proceeded to calculate its ellipticity. This he does nearly as follows:-

(1.) From Picart's and Cassini's measures of a degree, he finds, supposing the earth spherical, that its radius must be 19,615,800 Paris feet. From some observations on falling bodies at Paris, he calculates that the force of gravity at that place is such, that a body will fall 2174 lines in the first second of its descent. Knowing the earth's radius, and its time of rotation, it is easy to calculate the centrifugal force at the equator; viz., such that under its action, a body would describe 7.54064 lines in the first second. Since the resolved part of the centrifugal force perpendicular to the earth varies as the square of cosine of the latitude we can calculate the centrifugal force at Paris, and then adding it to the force of gravity, calculate as above, we find the whole undiminished force of gravity at that place to be such, that a body would describe 2177.267 lines in the first second of its descent. The undiminished force of gravity at the equator will differ from this by a very small quantity; hence rejecting small quantities of the second order, the ratio of centrifugal force at the equator to equatorial gravity is as 1 to 289 . This ratio is still in use.

(2.) If we took a spheroid, whose axes are as 101 to 100, by a simple application of Prop. XCI. Book I., Newton shows that the force of gravity at the pole is to that at the equator as 501 to 500 . Take now two canals, from the surface to the centre; let one meet the surface at the pole, the other at the circumference. That there may be equilibrium the weights of these two canals must be equal. Conceive these divided by transverse parallel equidistant surfaces into parts proportional to the wholes; the weights of any number of parts in the one leg will be to the weights of the same number of parts in the 
other as their magnitude and the accelerative forces of their gravity conjunctly; that is, as 101 to 100 , and 500 to 501 , or as $505: 501$. The difference, viz., four parts, must be supported by the centrifugal force. Hence the ratio of the centrifugal force bears to gravity the ratio $4: 505$.

(3.) Newton now brings in the rule of proportion. If a centrifugal force $\frac{4}{50} 5$ cause a difference of elevation of the two legs $\frac{1}{10} \overline{0}$, what difference will a centrifugal force $\frac{1}{2} \frac{1}{89}$ make? The calculation gives a result $\frac{1}{2} \frac{1}{2}$, or the diameter of the earth at the equator is to its diameter at the pole as 230 to 229 . The ratio of the difference of these diameters to the equatorial diameter, is called the ellipticity of the planet.

This investigation of Newton is manifestly altogether defective. He assumes not only that the spheroid is a form of equilibrium, but that the ellipticity is always proportional to the ratio of the centrifugal force to gravity. These two assertions are indeed true, but they are not self-evident. It was Maclaurin who first demonstrated their truth. It is very remarkable in how wonderful a manner Newton often arrives at correct results by means the most inadequate. Of this there are many other instances besides the present one. He guessed the mean density of the earth - he determined by analogy that the velocity of waves varied as the square root of their length. Another analogy led him to a curious result in regard to the tides.

2. Note III.

3. Newton remarks that the force of gravity will not bethe same at all points of the earth. For draw any radius $\mathrm{OP}=r$ from the centre to any point $\mathrm{P}$ in the circumN 2 
ference. Then the earth, being considered homogeneous, the attraction of the spheroid on any point $\mathrm{Z}$ in $\mathrm{O} \mathrm{P}$, resolved along this radius, will be proportional to its distance $\mathrm{OZ}$ from the centre. The same is true for the centrifugal force. Hence altogether the resolved part of gravity at any point $\mathrm{Z}$ in $\mathrm{O} \mathrm{P}$ resolved along the radius varies as $\mathrm{OZ}$. Let it be represented by $\omega z$, where $z=\mathrm{OZ}$, and $\omega$ may vary with the position of $\mathrm{P}$. The whole weight of the column is therefore

$$
\omega \int_{0}^{r} z d z=\frac{1}{2} \omega r^{2} \text {; }
$$

and this must be the same for all radii; hence $\omega$ varies inversely as $r^{2}$, and therefore the attraction varies as $\frac{z}{r^{2}}=\frac{1}{r}$, when $\mathrm{Z}$ lies on the surface. Hence the force of gravity towards the centre must vary on the surface of the same planet reciprocally as the distance of the bodies from the centre of the earth. As the earth is very nearly spherical, this must be also very nearly true for the whole force of gravity. Let $G$ then be the force of gravity at the equator, $g$ that at the point $\mathrm{P}$, whose latitude is $\lambda$ and radius $r$, then

$$
\begin{aligned}
g & =\mathrm{G} \frac{a}{r} \\
& =\mathrm{G} \frac{a}{b} \sqrt{1-e^{2} \cos ^{2} \lambda} \\
& =\mathrm{G}\left\{1+\varepsilon \sin ^{2} \lambda\right\} \text { nearly. }
\end{aligned}
$$

We shall show in another chapter that the force of gravity is proportional to the length of the seconds' pendulum; hence if $l$ and $\mathrm{L}$ be the lengths in the latitude $\lambda$ and at the equator,

$$
l=\mathrm{L}\left\{1+\varepsilon \sin ^{2} \lambda\right\} .
$$

The value of $l$ was known in the latitude of Paris, whence that of $\mathrm{L}$ can be found, and thence the length in 
any latitude. Newton now refers to a number of observations on the length of the seconds' pendulum in various latitudes, as a means of testing the truth of his theory. It appeared that the length of the seconds' pendulum does decrease as we approach the equator in the ratio of the square of the sine of the latitude, and so far observation confirms the theory. But it also appeared that the decrease of gravity was greater than that given by the theoretical expression. Whence Newton concluded that the value of $\varepsilon$, as given by theory, was a little too small. Here, however, he was wrong; for if the earth be considered as heterogeneous, an exactly opposite conclusion will follow from Clairaut's theorem.

The planet Jupiter, owing to its great angular velocity, is very protuberant at its equator, and thus the difference of the lengths of its two axes could be determined with tolerable accuracy. This planet, therefore, furnished Newton with a good test of the truth of his theory. Since the centrifugal force varies as the square of the angular velocity, and inversely as the radius; and gravity in different planets varies as the radius and as the density conjointly, hence the ratio of the centrifugal force to gravity varies as the square of the angular velocity directly and the density inversely. The ellipticity by what precedes has the same proportion. On substituting the known numerical values of these quantities, we find for the ellipticity of Jupiter $\frac{1}{9 \frac{1}{3}}$. Cassini observed in the year 1691, that the diameter of Jupiter from east to west is greater by about $\frac{1}{15}$ th part than the other diameter. Mr. Pound with his 123 feet telescope and an excellent micrometer, measured the diameters of Jupiter in the year 1719, and found them on four occasions to have the ratios 12 to $11,13 \frac{3}{4}$ to $12 \frac{3}{4}, 12 \frac{2}{3}$ to $11 \frac{2}{5}, 14 \frac{1}{2}$ to $13 \frac{1}{2}$. 
Thus Newton found a great discrepancy between the results of observation and theory. He accounts for this by remarking that there are two causes whose effects have not been allowed for. First, the diameters of Jupiter will appear in the telescope greater than they really are. The magnitude of the correction to be applied depends on the size of the telescope, and the care that has been taken in its construction. The greater diameter of Jupiter being $37^{\prime \prime}$, the lesser, according to the above ratio, will be $33^{\prime \prime} 25^{\prime \prime \prime}$, add thereto $3^{\prime \prime}$ for the effects of aberration, and the apparent diameters of the planet will be $40^{\prime \prime}$ and $36^{\prime \prime} 25^{\prime \prime \prime}$, which are to each other in the ratio of $11 \frac{1}{6}$ to $10 \frac{1}{6}$, very nearly. Secondly, Newton remarks that the theory assumed Jupiter to be of uniform density. But this is not likely to be true.
5.
6. Note IV.
8. 


\section{CHAPTER III.}

\section{THE Motion of A PARTICle in A REsistiNg Medium.}

1. The object and mode of conducting the enquiry.

2. When the resistance varies as the velocity.

a. Rectilinear motion.

$\beta$. Curvilinear motion. Section I.

3. When the resistance varies as the square of the velocity.

a. Rectilinear motion.

$\beta$. Curvilinear motion. Section II.

4. When the resistance varies partly as the velocity and partly as the square of the relocity, and the motion is rectilinear. Section III.

5. When the resistance varies as any power of the relocity and the motion is rectilinear - consideration of an analytical difficulty in the solution - the terminal velocity and instances.

6. The motion of a particle in a resisting medium round a centre of force.

a. The method used by Newton.

$\beta$. The method supplied by the Planetary Theory. Section IV.

1. PART of the theory of the motion of a body in a resisting medium is contained in the first four sections of the second book. The manner in which a medium resists the motion of a body moving in it is not the subject of this inquiry. It manifestly depends on a great many circumstances which we shall presently consider. At present we shall assume that the changes of resistance throughout the motion depend only on the changes of velocity. Again, the resistance will greatly depend on the form of the body, and will change, therefore, as the body during its motion opposes different faces to the resistance of the fluid. If the resultant of the resistances on the several parts of the 
body does not pass through the centre of gravity, the resistance itself will tend to cause rotation in the body. To simplify our analysis we shall consider the bodies to be indefinitely small particles.

The principle on which we proceed is, that the motion of the particle will be the same as if the resisting medium were removed, and that, first, a retarding force is substituted in its stead, which acts along a tangent to the motion of the particle, and depends only on the density of the fluid and the velocity of the particle at the moment under consideration, and that, secondly, all the impressed forces are, by the buoyancy of the fluid or other causes, diminished in a known ratio.

In considering the motions of bodies in vacuo we examined the effects of various laws of gravitation besides that which we know to exist in nature. So we may now examine the consequences of supposing the resistance to vary according to different functions of the velocity.

It is to Newton and Wallis * that we owe the first researches on the theory of the motion of bodies in resisting media. Wallis, in the same year that Newton published his "Principia," communicated his reflections on this subject to the Royal Society, who published them in their Transactions for 1687. Wallis, however, does not go so deeply into the subject as Newton did. A little after Newton's book appeared, Leibnitz asserted that he had already discussed these subjects, and that he had communicated his opinions twelve years previously to the Academy of Sciences at Paris. Huygens also considered some points in this theory at the end of Traité de la Pesanteur, which appeared in 1690. Finally, everything which had been either proved, or stated without proof, was demonstrated by Varignon by the aid of the modern calculus.

* Montucla, Part IV., Lib. VII. 6. 
The process that is now used to determine the motion is founded on the following reasoning. Let us suppose the particle moving in any curved line; let $s$ be the are described measured from any point at the time $t$. The time is supposed to be measured from any epoch anterior to the commencement of the motion. Then in the small time $\delta t$, the particle will, according to the notation of the differential calculus, describe a small arc $\delta s$, hence the mean velocity of the particle during this interval will be $\frac{\delta s}{\delta t}$. Now let $\delta t$ diminish without limit, the mean velocity will become the actual velocity $(v)$ at the instant $t$, and hence

$$
v=\frac{d s}{d t} .
$$

Similarly, the velocity being $v$ at the time $t$, that at the time $t+\delta t$ will be $v+\delta v$; hence the acceleration is such that in time $\delta t$ a velocity $\delta v$ has been added to the motion; hence the mean acceleration in that interval, measured by the velocity that would have been added in a unit of time if it had remained constant during that time, will be $\frac{\delta v}{\delta t}$. Now let $\delta t$ diminish without limit, and the mean acceleration becomes the actual acceleration $(f)$ at the instant, and

$$
\begin{aligned}
f & =\frac{d v}{d t} \\
& =\frac{d^{2} s}{d t^{2}}
\end{aligned}
$$

But an accelerating force is measured by the quantity of velocity it would add to the body in a unit of time, if it remained constant during that interval, so that we have merely to equate the accelerating force as given by the question, to the acceleration as given by the preceding 
formula, and we shall have an equation to determine the motion.

If $x$ and $y$ be the co-ordinates of the particle at any time $t$, it will follow by similar reasoning applied to the second law of motion that the velocities parallel to the axes are

$$
\frac{d x}{d t} \text { and } \frac{d y}{d t}
$$

and the accelerations are

$$
\frac{d^{2} x}{d t^{2}} \text { and } \frac{d^{2} y}{d t^{2}}
$$

parallel to the same axes.

2. Problem. To determine the motron of a particle moving in a straight line in a medium, and thereby resisted in the ratio of its velocity, and acted on by a uniform force.

Let $\mathrm{V}$ be the velocity of the particle at any time which we shall take as an epoch to measure our time from, and the place of the body at this time, as our "origin" from which to measure the distance of the particle at any other time. Take as our direction of measurement the direction in which the particle is moving at that epoch. Let $x$ and $v$ be the distance and velocity of the body at any time $t$. Let $m$ be its mass, and let $x v$ be the resistance when the body is moving with a velocity $v$; that is to say, let $x v$ be the moving force which would be required to keep the body moving, with this velocity in the medium. Let $f$ be the uniform accelerating force measured in the usual way. 
Then the whole moving force on the body will clearly be

$$
m f-x v \text {. }
$$

Also we know that for any particle the accelerating force is $\frac{d v}{d t}$, and therefore the moving force is $m \frac{d v}{d t}$. Equating these two

$$
\begin{aligned}
m \frac{d v}{d t} & =m f-\kappa v \\
\therefore \frac{d v}{d t} & =f-\frac{x}{m} \cdot v, \quad-\quad-\quad-
\end{aligned}
$$

This equation contains the whole of the motion.

First. We know that $v=\frac{d x}{d t}$, substituting, we have

$$
\frac{d v}{d t}=f-{ }_{m}^{x}-\frac{d x}{d t}
$$

$\therefore$ integrating throughout the time $t$,

$$
v-\mathrm{V}=f t-\frac{x}{m} x \quad-\quad-\quad-
$$

If $x$ were nothing, or the medium did not resist the particle, we should have,

$$
v-\mathrm{V}=f t \quad-\quad-\quad-\quad-
$$

Hence the motion lost by resistance is

$$
-\varkappa x
$$

that is, it is proportional to the space gone over. This is Newton's first proposition. A similar proof will apply if $f$ be not constant.

Secondly. Suppose $f=0$; or that the particle moves by its "vis insita" only. Then,

$$
\begin{aligned}
\frac{d v}{d t} & =-\frac{x}{m} \cdot v \\
\therefore \frac{d v}{v} & =-\frac{x}{m} d t
\end{aligned}
$$


integrating throughout the motion

$$
\begin{aligned}
& \log \frac{v}{\mathrm{~V}}=-\frac{x}{m} t \\
& \therefore v=\mathrm{V} \cdot \varepsilon^{-\frac{x}{m} t} \quad-\quad-\quad-
\end{aligned}
$$

That is, when the times are in arithmetical progression, the velocities are in geometrical progression. Also, we have already proved that

$$
v-\mathrm{V}=-\frac{x}{m} x \quad-\quad-\quad-
$$

or the velocity lost in passing over any space varies as that space.

As soon as the value of $x$ is known, the above formulæ may be submitted to accurate calculation. As its value depends on the form of the body, and the density of the medium, it can only be found by experiment in any particular case.

We may, however, learn some curious facts from these formula. From the formula for $v$, in terms of $t$, we see that though $v$ continuously decreases as $t$ increases, yet it never vanishes. The particle will then never stop, though constantly retarded. A little consideration will show that this is just what we should expect. For the resistance, varying as the velocity, takes away from the velocity in any small time $d t$, a certain fraction $\frac{x d t}{m}$ of the velocity that the particle has left. And as by taking away continually the halves of any quantity no one can remove the whole, so neither can this resistance ever destroy the whole velocity.

From the second formula we learn, that since $v$ can 
never be zero, it is always positive, and therefore

$$
x<\frac{m}{x} \mathrm{~V} \text {. }
$$

The resisted particle can never reach a point distant $\frac{m}{x} \mathrm{~V}$ from the origin, and it takes an infinite time to describe this space.

We may also represent the motion of the particle by the several parts of an hyperbola. Construct an hyperbola

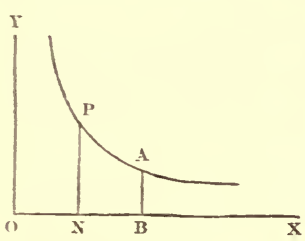

$\mathrm{P} A$, whose asymptotes are the perpendicular straight lines, $\mathrm{O}$ X, O Y. Then $P$ being any point, and $\mathrm{P} \mathrm{N}$ parallel to $\mathrm{OY}$, we know that $\mathrm{O} . \mathrm{N} \cdot \mathrm{P} \mathrm{N}$ is constant, and equal to one quarter the sum of the squares of the axes. Let the hyperbola be such that this is equal to $c^{2}$.

Then take $\mathrm{OB}=\mathrm{V}$, and $\mathrm{ON}=v$. By (5), we have,

$$
\mathrm{B} \mathrm{N}=\frac{\varkappa}{m} s .
$$

Hence the velocity being represented by $\mathrm{ON}$, the space described will be proportional to $\mathrm{B} \mathrm{N}$.

$$
\begin{aligned}
& \text { Also, } \mathrm{P} \mathrm{N}=y \\
& \begin{aligned}
d t & =-\frac{m}{x} \cdot \frac{d v}{v} \\
& =-\frac{m}{x c^{2}} y d v \\
\therefore t & =+\frac{m}{x c^{2}} \int_{\mathrm{v}}^{v} y d v,
\end{aligned}
\end{aligned}
$$

or the time is proportional to the area $\mathrm{PNBA}$. If the hyperbola be so drawn that the number of units of area 
in $c^{2}$ is equal to $\frac{m}{\chi}$, then the number of units of area in P N B A will equal the number of units of time elapsed. Thirdly. We may now proceed to consider the more general case. We have

$$
\frac{d v}{d t}=f-\frac{x}{m} v
$$

Hence by division we have,

$$
\frac{-d v}{\frac{f m}{x}-v}=-\frac{x}{m} d t
$$

Integrating throughout the motion,

$$
\begin{gathered}
\log \frac{\frac{f m}{x}-v}{\frac{f m}{x}-\mathrm{V}}=-\frac{x}{m} t \\
\therefore v-\frac{f m}{x}=\left(\mathrm{V}-\frac{f m}{x}\right)_{\varepsilon}^{-\frac{x}{m} \cdot t}
\end{gathered}
$$

Hence, if from the velocities there be subtracted the constant quantity $\frac{f m}{x}$, then these differences are in geometrical progression, when the times are in arithmetical progression.

Again, since $v=\frac{d x}{d t}$, this equation gives

$$
\frac{d x}{d t}=\frac{f m}{x}+\left(\mathrm{V}-\frac{f m}{\varkappa}\right)_{\varepsilon}-\frac{x_{t}}{m}
$$

integrating throughout the motion,

$$
x=\frac{f m}{x} \cdot t-\frac{m}{x}\left(\mathrm{~V}-\frac{f m}{x}\right)\left(\varepsilon^{-\frac{x}{m}}-1\right)
$$

which gives $x$ in terms of $t$. This equation is the same as the more simple one,

$$
x=\frac{m}{x}(f t-v+\mathrm{V})
$$

already established. 
From these equations we learn by interpretation several facts.

1. Let the velocity of projection be in the direction in which the accelerating force $f$ acts. Hence $f$ is here positive.

$\alpha$. Since $\varepsilon^{-\frac{z}{m} t}$ can never change sign, the quantities

$$
v-\frac{f m}{x} \text { and } \mathrm{V}-\frac{f m}{x}
$$

have always the same sign. Hence the velocity is always greater or always less than $\frac{f m}{x}$ according as the velocity of projection is greater or less than that quantity.

$\beta$. Since $\varepsilon^{-\frac{2}{m} t}$ continually diminishes as time goes on, but never vanishes for any finite value of $t$, hence the velocity $v$ continually approaches $\frac{f m}{x}$, but never actually equals it.

$\gamma$. If $V$ the velocity at any one point should equal $\frac{f m}{x}$, the velocity is always equal to the same quantity.

$\delta$. Since $v$ continually approaches a finite quantity, the expression for $x$ shows that the space described continually increases and finally becomes infinite in an infinite time.

$\varepsilon$. The velocity continually approaches the limit $\frac{f m}{x}$, hence $x$ is the mass of that body whose limiting velocity is $f$.

2. Let the velocity of projection be in a direction opposite to that in which the force $f$ acts. Here $f$ is negative, and our formula become

$$
\left.\begin{array}{r}
v+\frac{f m}{x}=\left(\mathrm{V}+\frac{f m}{x}\right) \varepsilon^{-\frac{x}{m} t} \\
x=\frac{m}{x}(-f t-v+\mathrm{V})
\end{array}\right\}
$$


As time goes on, the second factor on the right hand of the first equation continually decreases. Hence $v$ decreases, until a time comes when

$$
\frac{f m}{x}=\left(\mathrm{V}+\frac{f m}{x}\right) \varepsilon^{-\frac{\varkappa}{m} t}
$$

or,

$$
t=\frac{m}{x} \log \left(1+\frac{\mathrm{V} x}{f m}\right)
$$

then $v=0$ or the body comes to rest.

After this time, $v$ becomes negative, or the body begins to move in the opposite direction. This case has been already considered.

2. Problem. Supposing the force of gravity to be uniform and to tend perpendicularly to the plane of the horizon, to determine the motion of a projectile in a medium which resists in the ratio of the velocity.

Suppose the body projected with a velocity $\mathrm{V}$ and in a direction making an angle $\alpha$ with the plane of the horizon. Take this also as the epoch from which we measure the time, and let $v$ be the velocity and $\theta$ the angle the direction of motion makes with the horizon and $s$ the arc described at any other time $t$. Let the position of the particle be defined by two co-ordinates $x$ and $y$, giving respectively its distance from the point of projection measured along a horizontal line, and its altitude at the time $t$. Our first object is manifestly to find $x$ and $y$ in terms of $t$.

By the second law of motion each force produces its own effect as if the particle were at rest and it the only acting force. In considering then the motions parallel to any di- 
rection, we may wholly omit those perpendicular. The only force acting along the axis of $x$ is the resolved part of the resistance in that direction. This is

$$
-x v \cos . \theta
$$

But we know that $v=\frac{d s}{d t}$ and $\cos \theta=\frac{d x}{d s}$. Hence the moving force is

$$
-x \frac{d x}{d t}
$$

By similar reasoning that parallel to $y$ is

$$
-m g-\times \frac{d y}{d t}
$$

Hence just as in Art. 2., the equations of motion are

$$
\left.\begin{array}{r}
m \frac{d^{2} x}{d t^{2}}=-\times \frac{d x}{d t} \\
m \frac{d^{2} y}{d t^{2}}=-m g-\times \frac{d y}{d t}
\end{array}\right\}
$$

We have met with both these equations before. The motion parallel to $x$ is manifestly the same as that in the second case of Art. 2. We can therefore write down our results at once, viz.

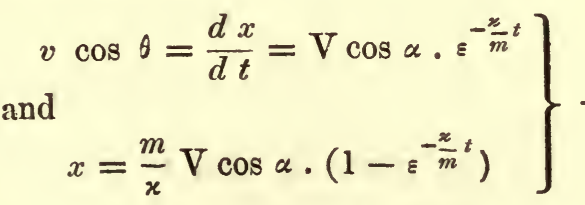

The motion parallel to $y$ is the same as that of the third case of Art. 2. Hence 


$$
\left.\begin{array}{l}
v \sin \theta=\frac{d y}{d t}=-\frac{g m}{x}+\left(\mathrm{V} \sin \alpha+\frac{g m}{\varkappa}\right) \varepsilon^{-\frac{\varkappa}{m} t} \\
y=-\frac{g m}{x} t+\frac{m}{x}\left(\mathrm{~V} \sin \alpha+\frac{g m}{x}\right)\left(1-\varepsilon^{-\frac{\varkappa}{m} t}\right)
\end{array}\right\}
$$

When any one of the five quantities $x, y, v, \theta, t$, are given, these four equations determine the other four. It is therefore reduced to be a mere matter of arithmetic calculation to determine the position of the particle at any time. It may be laborious and tedious, but there is no difficulty in it.

We shall now trace the curve the particle describes. Find $t$ from the second equation and substitute in the fourth, we have

$$
\begin{gathered}
y=\left(\tan \alpha+\frac{m}{x} \cdot \frac{g}{\mathrm{~V} \cos \alpha}\right) x \\
+\left(\frac{m}{x}\right)^{2} g \cdot \log \left(1-\frac{x}{m} \cdot \frac{x}{V \cos \alpha}\right) .
\end{gathered}
$$

Let $\mathrm{O}$ be the origin, $\mathrm{OB}$ the direction of projection,

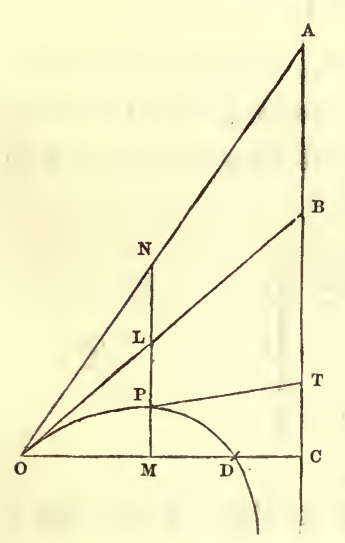

O C a horizontal. Take O C $=\frac{m}{x} \cdot \mathrm{V} \cos \alpha$, and draw $\mathrm{CB}$ vertical. Then from the above equation it is manifest that $\mathrm{BC}$ is an asymptote to the curve. Take B A $=\left(\frac{m}{x}\right)^{2} g$, a quantity, it will be observed, that is independent both of $\mathrm{V}$ and $\alpha$. Join $\mathrm{O} A$, and let $\beta=\angle \mathrm{AOC}$

$$
\therefore \tan \beta=\tan \alpha+\frac{m}{x} \cdot \frac{g}{V \cos \alpha} \text {. - }
$$


If $\mathrm{P}$ be any point in the curve, and N P M vertical

$$
\begin{aligned}
& \mathrm{MN}=\left(\tan \alpha+\frac{m}{x} \cdot \frac{g}{\mathrm{~V} \cos \alpha}\right) \cdot x \\
& \therefore \mathrm{N} \mathrm{P}=-\left(\frac{m}{x}\right)^{2} g \log \left(1-\frac{x}{m} \cdot \frac{x}{\mathrm{~V} \cos \alpha}\right) \\
& \text { put } \mathrm{N} \mathrm{A}=\xi, \mathrm{N} \mathrm{P}=\eta \\
& \therefore \eta=-\left(\frac{m}{x}\right)^{2} g \log \frac{\mathrm{C} \mathrm{M}}{\mathrm{C} \mathrm{O}} \\
&=-\left(\frac{m}{x}\right)^{2} g \log \frac{\xi}{a}-
\end{aligned}
$$

$$
\text { where } a=\frac{m}{x} \cdot \frac{\cos \alpha}{\cos \beta} \cdot V \text {. }
$$

This is a very simple form of the equation to the curve and enables us to investigate many of its properties with ease. We learn that if the successive values of $\mathrm{NA}$ are in geometric progression those of N P will be in arithmetical progression. This is Newton's second corollary.

It is also manifest from the manner in which we eliminated $t$, that we have

$$
\begin{gathered}
y=\left(\tan \alpha+\frac{m}{x} \cdot \frac{g}{\mathrm{~V} \cos \alpha}\right) x-\frac{g m}{x} \cdot t \\
\therefore \mathrm{N} \mathrm{P}=\frac{g m}{x} t ;
\end{gathered}
$$

or the particle moves in such a manner that its distance from $\mathrm{O} \mathrm{A}$, measured parallel to any fixed straight line, varies as the time. This is Newton's first corollary.

Since OB $\cdot \cos \alpha=\mathrm{OC}$, and $\mathrm{OC}=\frac{m}{x} \mathrm{~V} \cos \alpha$

$$
\therefore \mathrm{O} \mathrm{B}=\frac{m}{x} \mathrm{~V}
$$

and since any point may be considered as the origin of projection, we learn that the velocity at $\mathrm{P}$ is always pro- 
portional to the tangent P T. This is Newton's sixth corollary.

If $l$ be the latus rectum of the parabola that would be described under the same circumstances of projection, if the medium offered no resistance, then

$$
\begin{aligned}
l & =\frac{2 \mathrm{~V}^{2} \cos ^{2} \alpha}{g} \\
& =\left(\frac{x}{m}\right)^{2} \cdot \frac{\left.\overline{\mathrm{OC}}\right|^{2}}{g}
\end{aligned}
$$

3. Problem. To determine the motion of a particle moving in a straight line in a medium resisting in the ratio of the square of the velocity and acted on by a uniform force.

Let the symbols $\mathrm{V}, v, x, m, t, x$, have the same meaning that they had in the corresponding problem in which the resistance varied as the velocity. Then the whole moving force upon the particle will clearly be

$$
m f-x v^{2}
$$

also we have the accelerating force on a particle moving in any manner equal to $\frac{d v}{d t}$; which, since $v=\frac{d x}{d t}$, may also be put under the form $v \frac{d v}{d x}$. Taking both these forms, we have

$$
\left.\begin{array}{r}
m \frac{d v}{d t}=m f-x v^{2} \\
m v \frac{d v}{d x}=m f-x v^{2}
\end{array}\right\}
$$

which are identical equations. The first equation gives $v$ in terms of $t$, the second $v$ in terms of $x$.

First. Suppose $f=0$, or that the body moves by its "vis insita" only. 
Then since

$$
\begin{gathered}
\frac{d v}{d t}=-\frac{x}{m} v^{2} \\
\therefore-\frac{d v}{v^{2}}=\frac{x}{m} d t
\end{gathered}
$$

integrating throughout the motion

$$
\frac{1}{v}-\frac{1}{\mathrm{~V}}=\frac{x}{n} t \quad-
$$

Again

$$
\begin{aligned}
v \frac{d v}{d x} & =-\frac{x}{m} v^{2} \\
\therefore \frac{d v}{v} & =-\frac{x}{m} d x
\end{aligned}
$$

$\therefore$ integrating throughout the motion

$$
\begin{gathered}
\log \frac{v}{\mathrm{~V}}=-\frac{x}{m} x \\
\therefore v=\mathrm{V} . \varepsilon^{-\frac{x}{m} x} . \quad-
\end{gathered}
$$

Since $v=\frac{d x}{d t}$, this equation is the same as

$$
\varepsilon^{\frac{x}{m} x} d x=\mathrm{V} d t
$$

integrating throughout the motion

$$
\begin{gathered}
\varepsilon^{\frac{x}{m} x}-1=\frac{x}{m} \cdot \mathrm{V} t \\
\therefore x=\frac{m}{x} \cdot \log \left(1+\frac{x}{m} \cdot \mathrm{V} t\right) . \quad-
\end{gathered}
$$

From these equations we may gather every circumstance of the motion. From (1) we learn that if the times are in Arithmetical Progression the velocities are in Harmonical Progression; and that the velocity varies inversely as the time when counted from an era $\frac{m}{x \mathrm{~V}}$ units of time before 
the epoch at which the velocity is V. Also from (2) we learn that when the spaces are in Arithmetical Progression the velocities will be in geometrical Progression.

A mere inspection of these equations shows that the particle will continue to move for ever with a continually decreasing velocity, and that it will pass over an infinite space.

We may represent, as Newton has done, the motion by the several parts of an hyperbola. Construct the hyperbola $\mathrm{B} P$, whose asymptotes are the perpendicular straight lines $\mathrm{OX}, \mathrm{OY}$. Then $\mathrm{P}$ being any point in it and $\mathrm{P} \mathrm{N}$ an ordinate, we have

$$
\text { O N. } \mathrm{P} \mathrm{N}=c^{2}
$$

Take O A to contain $\frac{m}{\varkappa \mathrm{V}}$ units of space, and A $\mathrm{N}$ to contain $t$ units of space, then $A N$ represents the time.

$$
\begin{aligned}
& \text { Since } \mathrm{ON}=t+\frac{m}{x \mathrm{~V}} \\
& \therefore v=\frac{m}{x c^{2}} \cdot \mathrm{P} \mathrm{N},
\end{aligned}
$$

or the ordinate represents the velocity.

Since $x=\int v d t$

$$
\therefore x=\frac{m}{x c^{2}} \cdot \int y d t
$$

putting $y$ for $\mathrm{P} \mathrm{N}$, hence the area $A P$ represents the space described.

Secondly. We may proceed to consider the more general case. We have

$$
\begin{aligned}
& v \frac{d v}{d x}=f-\frac{x}{m} v^{2} \\
& \therefore \frac{v d v}{1-\frac{x}{m f} v^{2}}=f d x
\end{aligned}
$$


integrating throughout the motion,

$$
\begin{gathered}
\log \frac{1-\frac{x}{m f} v^{2}}{1-\frac{x}{m f} \mathrm{~V}^{2}}=-2 \frac{x}{m} x \\
\therefore v^{2}-\frac{m f}{x}=\left(\mathrm{V}^{2}-\frac{m f}{x}\right) \varepsilon-\frac{2 x}{m} \cdot x
\end{gathered}
$$

which is true, whether the body is moving in the direction in which $f$ acts or the opposite, provided only we give $f$ its proper sign.

Again we have

$$
\begin{gathered}
\frac{d v}{d t}=f-\frac{x}{m} v^{2} \\
\therefore \frac{d v}{1-\frac{x}{m f} v^{2}}=f d t \\
\therefore \frac{1}{2} \log \frac{1+\sqrt{\frac{x}{m f}} v}{1-\sqrt{\frac{x}{m f} v}} \cdot \frac{1-\sqrt{\frac{x}{m f}} \mathrm{~V}}{1+\sqrt{\frac{x}{m f}} \mathrm{~V}}=\sqrt{\frac{x f}{m}} \cdot t
\end{gathered}
$$

provided $f$ be positive, but if negative, say $-f^{\prime}$,

$$
\tan ^{-1} \sqrt{\frac{x}{m f}} v-\tan ^{-1} \sqrt{\frac{x}{m f}} \mathrm{~V}=-\sqrt{\frac{x f}{m}} \cdot t
$$

the first or second equation being true, according as the particle moves in the direction in which $f$ acts or the opposite.

From these equations we can infer the nature of the motion.

(1.) Let the velocity of projection be in the direction in which the force $f$ acts. Then $f$ is positive.

a. Since $\varepsilon^{-\frac{2 x}{m} x}$ can never change sign, the quantities

$$
v^{2}-\frac{m f}{x} \text { and } \mathrm{V}^{2}-\frac{m f}{x}
$$

○ 4 
have always the same sign. Hence the velocity is always greater or always less than $\sqrt{\frac{m f}{x}}$.

B. Since $\varepsilon^{-\frac{2 \pi}{m} x}$ continually decreases as $x$ increases, $v$ continually becomes more and more equal to $\sqrt{\frac{m f}{k}}$.

$\gamma$. As $t$ increases and finally becomes infinite, the equation connecting $v$ and $t$ shows that $v$ continually approaches and finally becomes equal to $\sqrt{\frac{m f}{k}}$, a fact which we could not infer from the first equation. This velocity is called the "terminal velocity." Let us represent it by the letter $u$. The equations may then be put under the simple forms

$$
\begin{gathered}
v^{2}-u^{2}=\left(\mathrm{V}^{2}-u^{2}\right) \varepsilon^{-\frac{2 f x}{u^{2}}} \\
\log \frac{u+v}{u-v}-\log \frac{u+\mathrm{V}}{u-\mathrm{V}}=2 \frac{f}{u} t .
\end{gathered}
$$

Suppose the particle to begin to fall from rest, then we have $\mathrm{V}=0$ and $f=g$.

$$
\begin{gathered}
v=u \cdot \sqrt{1-\varepsilon^{-\frac{2 g x}{u^{2}}}} \\
x=u t-\frac{u^{2}}{g} \log 2+\frac{u^{2}}{g} \log \left(1+\varepsilon^{-\frac{2 g}{u}}\right)
\end{gathered}
$$

(2.) Let the velocity of projection be in a direction opposite to that in which $f$ acts. Here $f$ is negative, write $-f$ for it, we learn that $v=0$, or the particle will come to rest after having described a space given by the equation

$$
\begin{aligned}
\frac{m f}{x} & =\left(\mathrm{V}^{2}+\frac{m f}{x}\right) \varepsilon-\frac{2 x}{m} x \\
\text { or, } x & =\frac{u^{2}}{2 f} \log \left(1+\frac{\mathrm{V}^{2}}{u^{2}}\right)
\end{aligned}
$$


and the time of describing this space is to be found from

$$
\sqrt{\frac{m}{x f}} \tan { }^{-1} \sqrt{\frac{x}{m f}} \mathrm{~V}=t,
$$

or,

$$
t=\frac{u}{f} \tan ^{-1} \frac{\mathrm{V}}{u} \text {. }
$$

In a preceding section Newton had determined the path of a projectile when the resistance varied as the velocity, and here was the place to give the solution of the corresponding problem, when the resistance varied as the square of the velocity. But this is a far harder question; we are even now unable to find quite accurately the path described. Newton considered the problem in an indirect manner. He determined the law of density that a given curve may be described, but he could not thence deduce the curve that gave the density uniform. He even made several mistakes, which were corrected at the suggestion of John Bernoulli, in the edition of 1713.* In 1718 Keill, in the course of the quarrel between the supporters of Newton and Leibnitz, dared the foreigners to attempt this question. Bernoulli was the first who gave a solution, and challenged the proposer to furnish his own solution within a certain time. This, however, Keill was unable to do. Meantime Nicholas Bernoulli, of Padua, supplied a solution; and seventeen days after the time fixed had elapsed, Taylor vindicated the honour of England by a tardy solution. The problem we shall now consider is somewhat more general than that enunciated by Newton, and it is as follows :- 
The resistance of the air being supposed to vary as the square of the velocity and as the density conjointly, and the forces to tend to directly to the plane of the horizon, to determine what must be the law of density of the medium that the particle may describe a given path, and to find the velocity at any point.

Let the axis of $x$ be taken horizontal and that of $y$ vertical, let $x, y$ be the co-ordinates of the particle at any time $t$, and $s$ the arc described. Let $\rho$ be the density of the medium at the point $(x, y)$, and $v$ the velocity of the particle, V the velocity, and $a$ the angle of projection. Then the resistance of the medium may be taken as

$$
\text { Resistance }=x \rho v^{2} \text {. }
$$

Let $\mathrm{Y}$ be the force acting on the particle parallel to the axis of $y$. The equations of motion will be

$$
\left.\begin{array}{l}
\frac{d^{2} x}{d t^{2}}=-\frac{x}{m} \rho v^{2} \frac{d x}{d s} \\
\frac{d^{2} y}{d t^{2}}=\mathrm{Y}-\frac{x}{m} \rho v^{2} \frac{d y}{d s}
\end{array}\right\}
$$

which may be put in the form

$$
\left.\begin{array}{l}
\frac{d^{2} x}{d t^{2}}=-\frac{x}{m} \rho v \frac{d x}{d t} \\
\frac{d^{2} y}{d t^{2}}=\mathrm{Y}-\frac{x}{m} \rho \frac{d y}{d t}
\end{array}\right\}
$$

Multiply these equations by $\frac{d y}{d t}$ and $\frac{d x}{d t}$ and subtract,

$$
\frac{d x}{d t} \frac{d^{2} y}{d t^{2}}-\frac{d y}{d t} \cdot \frac{d^{2} x}{d t^{2}}=\mathrm{Y} \frac{d x}{d t} \quad-
$$

By the theorem in the differential calculus for changing the independent variable, we have, therefore,

$$
\left.\overline{\frac{d x}{d t}}\right|^{2} \cdot \frac{d^{2} y}{d x^{2}}=\mathrm{Y}
$$


But from (1) we have

$$
\begin{aligned}
& \frac{{ }_{d x}{ }^{-1}}{d t} d \frac{d x}{d t}=-\frac{x}{m} \rho d s \\
& \therefore \log \frac{d x}{d t},=\mathrm{C}-\frac{x}{m} \int \rho d s,
\end{aligned}
$$

whence eliminating $\frac{d x}{d t}$, we have

$$
\frac{d^{2} y}{d x^{2}}=\frac{\mathrm{Y}}{\mathrm{V}^{2} \cos ^{2} \alpha} \cdot \varepsilon \cdot \frac{2 x}{m} \int_{o}^{s} s^{d s}
$$

an equation from which either $\rho$ or $Y$ may be found when the relation between $x$ and $y$, which determines the curve is given.

The velocity at any point of the curve is that due to onefourth the chord of curvature. For looking at equation (3), the left-hand side is the denominator of the expression for the radius of curvature $R$, whence

$$
\begin{gathered}
\overline{v^{2}}=\mathrm{Y}_{\frac{d x}{d s}} \\
\text { or } v^{2}=2 \mathrm{Y} \cdot\left(\frac{1}{2} \mathrm{R} \frac{d x}{d s}\right)
\end{gathered}
$$

Since $\frac{d x}{d s}$ is the cosine of the angle, the normal makes with the axis of $y$, the quantity in brackets is one fourth the chord of curvature. Whence the proposition follows.

The equation (4) will also enable us to determine the equation to the path when the law of density and the force is given; as an instance take $\rho$ constant, and $\mathrm{Y}$ $=-g$ the force of gravity. Then

$$
\frac{d^{2} y}{d x^{2}}=-\frac{g}{\mathrm{~V}^{2} \cos ^{2} \alpha} \cdot \varepsilon^{2^{2}} \xi^{s}
$$

an equation which can be only approximately integrated.

Newton takes several examples to illustrate his reasoning. For instance, if the path be a semicircle and the force gravity, we have 


$$
\left.\begin{array}{c}
x^{2}+y^{2}=a^{2} \\
\mathbf{Y}=-g
\end{array}\right\}
$$

where $\alpha$ is the radius of the semicircle, whence, by a simple substitution, we get

$$
\rho=-\frac{3}{2 x a} \cdot \frac{x}{y}
$$

so that the density of the medium at any point varies as tangent of the angle a radius through the point makes with the vertical. Newton also determines the law of density when the particle describes an hyperbola with one asymptote vertical, chiefly with the view of finding an approximation to the curve which a particle will describe in a uniformly resisting medium. This was a problem which Newton was unable to solve, except in this imperfect and indirect manner. We shall not therefore dwell on this, but will proceed at once to indicate the manner in which the question is now answered.

Taking the equation

$$
\frac{d^{2} y}{d x^{2}}=-\frac{g}{\mathrm{~V}^{2} \cos ^{2} \alpha} \cdot \varepsilon^{2 \frac{x}{m} s}
$$

multiply both sides by $\sqrt{1+p^{2}} d x$, where $p=\frac{d y}{d x}$, and integrate

$$
\frac{\varepsilon^{2 \frac{\varkappa}{m} s}-1}{2 \frac{x}{m}}=-\frac{\mathrm{V}^{2}}{2 g} \cos ^{2} \alpha \cdot\left\{p \sqrt{1+p^{2}}\right.
$$

$$
+\log \left(p+\sqrt{\left.1+p^{2}\right)}+c\right\}
$$

In gunnery $p$ is usually small, let us reject the powers of $p$ higher than the second, we get

$$
\varepsilon^{2 \frac{z}{m} s}=1+\frac{2 x V^{2}}{m g} \cos ^{2} \alpha(\tan \alpha-p)
$$




$$
\begin{gathered}
\therefore \frac{d p}{d x}=-\frac{g}{\mathrm{~V}^{2} \cos ^{2} \alpha}-2 \frac{x}{m}(\tan \alpha-p) \\
\therefore \frac{d p}{d x}-2 \frac{k}{m} \cdot p=-\left(2 k \tan \alpha+\frac{g}{\mathrm{~V}^{2} \cos ^{2} \alpha}\right) \\
\therefore p=\tan \alpha-\frac{m g \sec ^{2} \alpha}{2 k \mathrm{~V}^{2}} \cdot\left(\varepsilon^{2 k x}-1\right) \\
\therefore y=x\left(\tan \alpha+\frac{m g \sec ^{2} \alpha}{2 k \mathrm{~V}^{2}}\right)-\frac{m^{2} g\left(\varepsilon^{2} \frac{k}{m}-1\right)}{4 k^{2} \mathrm{~V}^{2} \cos ^{2} \alpha}
\end{gathered}
$$

which is the equation to the path.*

4. Рroblem. To determine the motion of a particle moving in a straight line in a medium resisting partly in the ratio of the velocity, and partly in the ratio of the square of the velocity, and acted on by a uniform force.

Let the symbols $\mathrm{V}, v, x, m, t, f$ have the same meaning that they had in the corresponding problem in which the resistance varied as the velocity. Let the whole resistance $\mathrm{R}$ be represented by the formula

$$
\mathrm{R}=x v+\frac{x}{\alpha} v^{2}
$$

then the whole moving force will then be

$$
m f-x v-\frac{x}{\alpha} v^{2}
$$

and our equations of motion are

$$
\left.\begin{array}{r}
m \frac{d v}{d t} \\
m v \frac{d v}{d x}
\end{array}\right\}=m f-x v-\frac{x}{\alpha} v^{2} .
$$

First. Let $f=o$ or the particle move by its innate force only.

Then

$$
\begin{gathered}
\frac{d v}{d t}=-\frac{x}{m} v-\frac{x}{m} \cdot \frac{v^{2}}{\alpha} \\
\text { * Earnshaw's Dynamics. }
\end{gathered}
$$




$$
\frac{d v}{\left(v+\frac{\alpha}{2}\right)^{2}-\frac{\alpha^{2}}{4}}=-\frac{x}{m} \cdot \frac{d t}{\alpha}
$$

integrating throughout the motion

$$
\begin{gathered}
\log \frac{1+\frac{\alpha}{v}}{1+\frac{\alpha}{\bar{V}}}=+\frac{x}{m} \cdot t \\
\therefore \frac{1}{v}+\frac{1}{\alpha}=\left(\frac{1}{\bar{V}}+\frac{1}{\alpha}\right) \varepsilon^{+\frac{x}{m} t}
\end{gathered}
$$

So that when the times are in Arithmetical Progression, quantities reciprocally proportional to the velocities, increased by a certain given quantity will be in Greometrical Progression. This is Newton's eleventh proposition.

Again, since

$$
\begin{gathered}
v \frac{d v}{d x}=-\frac{x}{m} v-\frac{x}{m} \cdot \frac{v^{2}}{a} \\
\frac{d v}{v+a}=-\frac{x}{m} \frac{d x}{a}
\end{gathered}
$$

integrating throughout the motion

$$
\begin{array}{r}
\log \frac{v+\alpha}{V+\alpha}=-\frac{x}{m} \cdot \frac{x}{\alpha} \\
\therefore v+\alpha=(V+\alpha) \varepsilon^{-\frac{z}{m} \cdot \frac{x}{\alpha} .}
\end{array}
$$

So that if the spaces described are taken in Arithmetical Progression, the velocities augmented by a certain given quantity will be in Geometrical Progression. This is Newton's twelfth proposition. And by eliminating $v$ between the two equations, we can find $x$ in terms of $t$. But the result is complicated and of little value.

Secondly. Let us proceed to the more general case: we have

$$
\frac{d v}{d} t=f-\frac{x}{m} v-\frac{x}{m} \cdot \frac{v^{2}}{\alpha}
$$




$$
\therefore\left(\frac{d v}{\left.v+\frac{\alpha}{2}\right)^{2}-\left(\frac{\alpha^{2}}{4}+\frac{m f \alpha}{x}\right)}=-\frac{k}{m} \cdot \frac{d t}{\alpha}\right.
$$

for the sake of brevity, put

$$
c^{2}=\frac{\alpha^{2}}{4}+\frac{m f \alpha}{k},
$$

and integrating, we get, after correction,

$$
\log \frac{v+\frac{\alpha}{2}+c}{v+\frac{\alpha}{2}-c}-\log \frac{\mathrm{V}+\frac{\alpha}{2}+c}{\mathrm{~V}+\frac{\alpha}{2}-c}=2 \frac{c}{\bar{d}} \cdot \frac{k}{m} t,
$$

this will enable us to find $t$ in terms of $v$, and, by solving the equation, $v$ in terms of $t$.

If the particle be moving in the direction opposite to that in which $f$ acts, and if, also

$$
m f>\frac{x a}{4},
$$

the above integral becomes imaginary; the true expression will manifestly be, if

$$
\begin{gathered}
b^{2}=\frac{m f \alpha}{x}-\frac{\alpha^{2}}{4} \\
\tan ^{-1} \frac{v+\frac{\alpha}{2}}{b}-\tan ^{-1} \frac{\mathrm{V}+\frac{\alpha}{2}}{b}=-\frac{b}{a} \frac{x}{m} t
\end{gathered}
$$

Again, we have

$$
\begin{gathered}
v \frac{d v}{d x}=f-\frac{x}{m} v-\frac{x}{m} \cdot \frac{v^{2}}{\alpha} \\
\therefore \frac{v d v}{v+\left.\frac{\alpha}{2}\right|^{2}-\left(\frac{\alpha^{2}}{4}+\frac{m f \alpha}{x}\right)}=-\frac{x}{m} \cdot \frac{d x}{\alpha}
\end{gathered}
$$

for the sake of brevity put

$$
c^{2}=\frac{\alpha^{2}}{4}+\frac{m f \alpha}{x}
$$


and integrating we have

$$
\left.\log \left(\overline{v+\frac{\alpha}{2}}\right)^{2}-c^{2}\right)+\frac{\alpha}{2 c} \log \frac{v+\frac{\alpha}{2}+c}{v+\frac{\alpha}{2}-c}=\mathrm{C}-\frac{2 x}{m \alpha} \cdot x
$$

where the quantity $\mathrm{C}$ is obviously equal to what the left hand side of this equation becomes when $\mathrm{V}$, the initial velocity, is substituted for $v$.

If the particle move in the direction opposite to that in which $f$ acts, and if

$$
m f>\frac{x \alpha}{4}
$$

this expression becomes imaginary. It is obvious, however, that if we put

$$
b^{2}=\frac{m f \alpha}{x}-\frac{\alpha^{2}}{4}
$$

that the true integral will be

$$
\left.\log \overline{\left(v+\frac{a}{2}\right)^{2}}+b^{2}\right)-\frac{\alpha}{b} \tan ^{-1} \frac{v+\frac{\alpha}{2}}{b}=\mathrm{C}-\frac{2 x}{m} \cdot \frac{x}{\alpha}
$$

where $\mathbf{C}$ is obviously equal to what the left hand side becomes when $\mathrm{V}$ is put for $v$.

The quantity $x$ in these formulx is the mass of that particle whose terminal velocity is

$$
\sqrt{\frac{a^{2}}{4}+\alpha g}-\frac{\alpha}{2}
$$

the quantity $\frac{x}{m}$, therefore, represents a number, thus we see that the preceding expressions are perfectly homogeneous.

In exactly the same manner we may proceed to determine the motion of a particle in a medium resisting according to any other function of the velocity. 
If the resistance vary as $v^{n}$ and the particle nnove by its own "vis insita" only. Then since

$$
\frac{d v}{d t}=v \frac{d v}{d x}=-\frac{x}{m} \cdot v^{n}
$$

$\therefore$ integrating both equations throughout the motion,

$$
\left.\begin{array}{l}
\frac{1}{v^{n-1}}-\frac{1}{\mathrm{~V}^{n-1}}=(n-1) \cdot{ }_{m}^{x} \cdot t \\
\frac{1}{v^{n-2}}-\frac{1}{\mathrm{~V}^{n-2}}=(n-2) \cdot \frac{x}{m} \cdot x
\end{array}\right\}
$$

equations which never become nugatory except when $n=1$ or $n=2$, both which cases have been already considered.

From these equations we may learn several remarkable facts.

First. Suppose $n$ greater than 2. Then both the right hand members of the above equations are positive; hence $v$ can never vanish, and the body will continue moving for ever, with an ever diminishing velocity, and will pass over an infinite space.

Secondly. Suppose $n$ greater than unity but less than 2 . Then $v$ vanishes only when $t$ is infinite, but then

$$
x=\frac{m}{x} \cdot \frac{1}{2-n} \cdot \frac{1}{\mathrm{~V}^{n-2}}
$$

So that the particle $t$ continues to move always with an ever diminishing velocity, and will pass over a finite space.

Thirdly. Suppose $n$ less than unity, then when $v$ vanishes, we have

$$
\left.\begin{array}{l}
t=\frac{m}{x} \cdot \frac{1}{1-n} \cdot \frac{1}{\mathrm{~V}^{n-1}} \\
x=\frac{m}{x} \cdot \frac{1}{2-n} \cdot \frac{1}{\mathrm{~V}^{n-2}}
\end{array}\right\}
$$

that is, the particle moves on with a diminished velocity, 
and finally stops after a time given by the first equation, having described a space given by the second. It is manifest that the particle remains at rest, until it is disturbed by some new force.

But here we have a remarkable singularity in the equations; for according to them, as $t$ increases $v^{1-n}$ does not remain equal to zero, but becomes negative. What is the explanation of this? It must be sought for in the nature of a differential equation. There are always two species of integrals. One called the "general integral," which contains the full number of arbitrary constants, and another, called the "singular solution," not included in the former, and which does not contain the full number of arbitrary constants. These latter in dynamical problems are usually of little value, because they do not agree with the initial conditions of motion. But, if by any chance they should satisfy these conditions, it is possible that they may be the true representatives of the subsequent motion. The choice between them and the general integral must be founded on extrinsic considerations. The differential equation we started with, is a mere statement of the forces, and must be true throughout the motion. This motion must therefore be represented either by the general or the singular solution. We have seen that the general solution only represents the motion up to a certain time; after that we must have recourse to the singular solution. If we proceed to find this, by the usual methods, we arrive at the solution

$$
v=0 \text {, }
$$

which we see represents the motion subsequently to the above mentioned time.*

One of the most remarkable facts connected with motion in a resisting medium is the existence of a "terminal ve-

* Duhamel, Cours de Mécanique. 
locity" when the body moves in a straight line, under the action of a uniform force. This may be defined to be that velocity which makes the resistance equal to the moving force acting on the particle. Let $\phi(v)$ be the law of resistance, and $m g$ the moving force. Then if $u$ be the terminal velocity, we have

$$
\phi(u)=m g
$$

an equation to find $u$. Suppose the resistance to vary as the square of the velocity and $\phi(v)=x v^{2}$, then

$$
u=\sqrt{\frac{m g}{x}}
$$

It is manifest that if the body were projected with this velocity, it would continue to move uniformly in the medium. This is a consequence of the first law of motion. Also if the particle began to fall from rest, its velocity will continually increase under the action of the force $g$, and approach equality with the "terminal" velocity, and only become equal to it when the time is infinite. All this is quite manifest and needs no analytical investigation.

Though the velocity of the particle never becomes equal to the terminal velocity, yet it soon becomes so little different from it that, for all practical purposes, we may consider the particle as moving with an uniform velocity equal to the terminal velocity. In considering the motion of a falling body we arrive at the equation,

$$
v^{2}-\frac{m f}{x}=\left(\mathrm{V}^{2}-\frac{m f}{x}\right) \cdot \varepsilon^{-\frac{2 x}{m} \cdot x}
$$

Now if $\frac{m}{x}$ be a very small quantity, it does not require a very large value of $x$ to render the second factor so small that we may without much error consider

$$
v^{2}=\frac{m f}{x} \text {. }
$$


It is needless to point out how different this is from motion in vacuo, where the velocity would have gone on increasing without any limit.

One familiar instance of motion in a resisting medium is the descent of rain. The drops descend then with a uniform motion, the larger drops going quicker than the smaller, and the velocity of descent increasing as the drops grow in size. If the rain descended with the velocity due simply to the action of gravity, a heavy shower of rain would commit serious injury. A drop of rain falling from a cloud a mile high would have acquired a velocity of about 576 feet a second. The actual velocity is perhaps less than one five thousandth part of this.

In the fourth section Newton discusses the motion of a particle in a resisting medium when acted on by a feebly resisting medium. He begins by considering the case in which the density of the medium varies inversely as the distance from the centre of force, and under peculiar conditions of the motion of the body extends his deductions to any law of distance. We have followed his method, with the exception that, as we have the powerful aid of analysis, we can treat the question with greater generality. But since Newton's time we have discovered much better methods; it has been thought not out of place to give a very brief view of them, so far as they depend only on first principles.

6. a. A particle moves in an equiangular spiral under the action of a central force in the pole, in a medium whose density varies as some function of the distance from the pole. To determine the connexion between the law of density and the law of force that this motion may be possible.

The equiangular spiral, by definition, possesses the property that the tangent at any point makes a constant 
angle with the radius vector: let this angle be called $\alpha$. Let $r$ and $v$ be the radius vector and velocity of the particle at any time $t$. Let $P$ be the central force, and $x v^{2}$ the resistance at any time, then $P$ and $x$ are functions of $r$.

The equation giving the motion along the arc is clearly

$$
\frac{d v}{d t}=-x v^{2}-\mathrm{P} \cos \alpha .
$$

But in all curves

$$
\begin{gathered}
\frac{d r}{d t}=v \cos \alpha \\
\therefore \frac{d v}{d t}=\frac{d v}{d r} \cdot v \cos \alpha
\end{gathered}
$$

Hence the above equation becomes

$$
\frac{d v^{2}}{d r}+\frac{2 x}{\cos \alpha} \cdot v^{2}=-2 \mathrm{P} \quad-\quad-
$$

It is to be observed that this equation is true whatever be the nature of the curve described.

The equation giving the motion perpendicular to the arc is well known to be

$$
\overline{\mathrm{R}}=\mathrm{P} \operatorname{Sin} \alpha
$$

But in the equiangular spiral the radius of curvature $R$ is $\frac{r}{\operatorname{Sin} a}$, hence we have

$$
v^{2}=\mathrm{Pr} \quad \text { - }
$$

If we substitute this in equation (1) we get

$$
\begin{aligned}
\frac{1}{\mathrm{P}} d \mathrm{P} & =-\left(3+\frac{2 \varkappa r}{\cos \alpha}\right) \frac{d r}{r} \\
\therefore \log \mathrm{P} & =\mathrm{C}-\int\left(3+\frac{2 \varkappa r}{\cos \alpha}\right) \frac{d r}{r}
\end{aligned}
$$

which is the required connection.

If the central force vary inversely as the Square of the distance, we have 


$$
\mathrm{P}=\frac{\mu}{r^{2}}
$$

whence it follows that

$$
x=-\frac{\cos \alpha}{2} \cdot \frac{1}{r}-\quad-(3) \text {. }
$$

or the density varies inversely as the distance. The negative sign shows that the angle $\alpha$ must be greater than a right angle, or that the particle continually approaches the centre of force. Let $x=\frac{\mathrm{D}}{r}$, where $\mathrm{D}$ is the resistance at a unit of distance to a unit of velocity, then

$$
\mathrm{D}=\frac{-\cos \alpha}{2} \text {. }
$$

On looking at equation (2) we see that whatever be the law of force, the velocity is the same as that in a circle at the same distance, and when the force varies inversely as the Square of the distance, we have

$$
v=\sqrt{\frac{\mu}{r}}
$$

This is Newton's first corollary.

Again, when $x$ or $D$ is given, equation (3) gives us the means of finding $\alpha$. Thus a spiral may be fitted to any density. This is Newton's second corollary.

And when the $\alpha$ or the spiral is given, the ratio of the resistance to the centripetal force is easily found. We observe that since cos. $\alpha$ must be less than unity, this ratio must be less than $\frac{1}{2}$, otherwise the orbit described will not be the equiangular spiral. When this ratio is $\frac{1}{2}$ exactly, the value of $\alpha$ is zero; that is, the spiral is reduced to its limiting case, viz., a straight line passing through the pole. This includes Newton's third and fourth corollaries.

Also the time of describing any arc may be found; for since 


$$
\begin{aligned}
\frac{d r}{d t} & =v \cos \alpha \\
& =\sqrt{\frac{\mu}{r} \cos \alpha} \\
\therefore t & =\frac{1}{\cos \alpha} \cdot \frac{1}{\sqrt{ } \mu} \int \sqrt{ } r d r
\end{aligned}
$$

So that the time of going from the distance $r_{1}$ to the distance $r_{2}$ is

$$
\mathrm{T}=\frac{2}{3 \sqrt{\mu}} \frac{1}{\cos \alpha}\left(r_{2}^{\frac{3}{2}}-r_{1}^{\frac{3}{2}}\right)-
$$

When $\alpha$ is nearly a right angle, this time becomes very long. If $\alpha=0$, the same formula will give the time of descent $\mathrm{T}^{\prime}$ down any part of a radius vector. Hence we see that

$$
\mathrm{T}=\frac{\mathrm{T}^{\prime}}{\cos \alpha^{\prime}}
$$

and the number of revolutions described may also be found: for if $\theta$ be the angle the radius vector $r$ makes with any fixed straight line, we have

$$
\begin{aligned}
d \theta & =\frac{d r}{r} \tan \alpha \\
\therefore \theta_{2}-\theta_{1} & =\tan \alpha \log \frac{r_{2}}{r_{1}}
\end{aligned}
$$

Hence the number of revolutions will be

$$
\mathrm{N}=\frac{\tan \alpha}{2 \pi} \log \frac{r_{2}}{r_{1}} \quad-\quad-
$$

This includes Newton's fifth and sixth corollaries.

If we neglect the eccentricities of the planetary orbits, the velocity at any point in vacuo is given by the usual formula

$$
v^{2}=P r
$$

Let us then assume as the velocity in a resisting medium

$$
v^{2}=\operatorname{Pr}_{\mathbf{P}_{4}}(1+p)
$$


where $p$ is a very small quantity. Then since the equation

$$
v^{2}=\mathrm{P} \text { R Sin. } \alpha
$$

is always true, we have

$$
\text { R Sin. } \alpha=r(1+p) \text {. }
$$

Substitute for $\mathbf{R}$ and Sin $\alpha$ their known values

$$
\frac{d s}{d \beta} \cdot \frac{r d \theta}{d s}=r(1+p) \text {, }
$$

where $\beta$ is the angle the tangent makes with any fixed straight line,

$$
\begin{gathered}
\therefore \frac{d \theta}{d \beta}=1+p \\
\therefore \beta-\theta=\int \frac{p d \theta}{1+p}+\text { constant. }
\end{gathered}
$$

But $\beta-\theta=\alpha$, hence the variation of $\alpha$ is expressed by the above integral. As an approximation, consider $p$ as a small constant whose square may be rejected, hence

$$
\alpha=\alpha_{0}+p \theta
$$

Now $p$ is so small that it requires a large value of $\beta$, the angle described, to render the latter term sensible. Hence for many revolutions we may regard $\alpha$ as constant, that is, regard the orbit as an equiangular spiral. We may, therefore, apply our preceding conclusions. If the radius of a planet's orbit be $r$, by equations (3) (4) (5) we learn that the time before the radius has decreased by $\delta r$ will be

$$
\mathbf{T}=\frac{1}{2 \sqrt{\mu}} \frac{\delta r}{x r^{\frac{1}{2}}},
$$

and the number of revolutions in that time will be

$$
\mathrm{N}=\frac{1}{4 \pi} \cdot \frac{\delta r}{x r^{2}} \text { nearly. }
$$

The value of $x$ is so small that, as these formulæ show, 
it will require a very long time before $r$ can be perceptibly changed.

But regarding $\delta r$ as indefinitely small, these expressions will be accurate. Hence the whole time which it will take the planet to arrive at the centre will be

$$
\tau^{\prime}=\frac{1}{2 \sqrt{ } \mu} \cdot \int \frac{d r}{\varkappa r^{\frac{1}{2}}},
$$

and the whole number of revolutions will be

$$
\mathrm{N}^{\prime}=\frac{1}{4 \pi} \int \frac{d r}{x r^{2}} \text { nearly. }
$$

These expressions cannot be integrated until the law of density is known as a function of the distance.

If we assume that the density varies inversely as the distance from the centre, we have $x r=\mathrm{D}$, a constant; performing the integrations, we have

$$
\begin{aligned}
\tau^{\prime} & =\frac{r^{\frac{3}{2}}}{3 \sqrt{\mu} \mathrm{D}} . \\
\mathrm{N}^{\prime} & =\infty .
\end{aligned}
$$

The number of revolutions is infinite, because the time of a revolution becomes ultimately infinitely small. Compare the first of these formulæ with (4) and we learn that if $r_{1}$ be the radius at any instant, $r_{2}$ the radius after one complete revolution, the whole time of reaching the centre of force will be to one revolution from radius $r_{1}$ to radius $r_{2}$ in the ratio of $r_{1}^{\frac{3}{2}}$ to $r_{1}^{\frac{3}{2}}-r_{2}^{\frac{3}{2}}$ or $\frac{2}{3} r_{1}$ to $r_{1}-r_{2}$ nearly. In the case of the planets, $r_{1}-r_{2}$ is so small as to be altogether insensible; hence the above time is indefinitely great.

$\beta$. In the seventeenth proposition of the third section of the first book Newton remarks, that " if a body move in a conic section, and is forced out of its orbit by any impulse, we can discover the orbit in which it will afterwards pursue 
its course." " And if that body is continually disturbed by the action of some foreign force, we may nearly know its course by collecting the changes which that force introduces in some points, and estimating the continual changes it will undergo in the intermediate places from the analogy that appears in the course of the series." This method, which Newton only applies to determine the general effect of any disturbing force, we can use, by the aid of the differential calculus, to determine its effect to any degree of accuracy.

Let us conceive a planet to be describing an ellipse round the sun in the focus, and let it be continually disturbed by the resistance of the medium in which the planet moves. At the time $t$, let $a, e, w$ be the mean distance, eccentricity, and longitude of the apse of the ellipse, and let $r, \theta, v$ be the distance, longitude, and velocity of the planet. The attraction of the sun and the resistance of the medium will be represented by $\frac{\mu}{r^{2}}$ and $x v^{2}$, where $\mu$ and $x$ are certain constants.

From the sixteenth proposition of the third section of the first book it is easy to deduce by a known property of the ellipse, that

$$
\begin{aligned}
& v^{2}=\mu\left(\frac{2}{r}-\frac{1}{a}\right)-\quad-\quad(1) . \\
& \therefore \frac{1}{a}=\frac{2}{r}-\frac{v^{2}}{\mu} .
\end{aligned}
$$

But in the time $d t, v$ is decreased by the resisting medium by

$$
\begin{aligned}
-d v & =x v^{2} d t \\
\therefore-\frac{d a}{a^{2}} & =-\frac{2 v}{\mu} d v \\
& =+\frac{2 v^{3} x}{\mu} d t
\end{aligned}
$$


we might express $v$, the velocity, in terms of the time by the usual formulæ of elliptic motion; this would not lead to any lengthy calculations, but as $e$ and $x$ are very small, there is no practical advantage in investigating more than the principal terms in the series expressing the changes of the elements. We may then put

$$
v=a n \text {, }
$$

where $n$ is the mean angular velocity; and hence $\mu=a^{3} n^{2}$,

$$
\therefore \frac{d a}{d t}=-2 \times a^{2} n
$$

and $x$ being very small, we may reject the variations of the quantities on the right hand side,

$$
\therefore a_{1}-a_{0}=-2 \times a^{2} n t \text {, }
$$

where $\alpha_{0}, a_{1}$, are the values of the mean distance at the beginning and end of the interval $t$.

By the fourteenth proposition of the third section and the first of the second, we can easily see that

$$
\mu a\left(1-e^{2}\right)=v^{2} r^{2} \sin ^{2} \alpha,
$$

where $\alpha$ is the angle between the radius vector and tangent. Hence

$$
\begin{aligned}
\mu \frac{d a}{d t}\left(1-e^{2}\right)-2 \mu a e \frac{d e}{d t} & =2 v \sin ^{2} \alpha r^{2} \frac{d v}{d t} \\
& =-2 x v^{3} \sin ^{2} \alpha \cdot r^{2}
\end{aligned}
$$

Now as we retain only the principal terms, we have

$$
\begin{gathered}
\alpha=90^{\circ}, \\
r=a(1-e \cos n t),
\end{gathered}
$$

substituting, we get

$$
\begin{gathered}
\frac{d e}{d t}=-2 \times a n \cos n t, \\
\therefore e_{1}-e_{0}=-2 x a \cdot \sin n t,
\end{gathered}
$$

where $e_{0} e_{1}$ are the values of $e$ at the beginning and end of 
the interval $t$. Thus we learn that in one complete revolution the eccentricity is unchanged.

By a well known property of the ellipse

$$
\begin{aligned}
\frac{a\left(1-e^{2}\right)}{r} & =1+e \cos (\theta-\omega) \\
\therefore \frac{r \cdot v^{2} \sin ^{2} \alpha}{\mu} & =1+e \cos (\theta-\omega)
\end{aligned}
$$

putting $\sin \alpha=1$ we get by differentiating

$$
-\frac{2 r v}{\mu} \frac{d v}{d t}=\frac{d e}{d t} \cos (\theta-\omega)+e \sin (\theta-\omega) \frac{d \omega}{d t},
$$

so that

$$
\begin{gathered}
\frac{d \omega}{d t}=-\frac{2 x a n}{e} \sin n t \\
\therefore \omega_{1}-\omega_{0}=\frac{2 x a}{e} \cdot \cos n t,
\end{gathered}
$$

where $\omega_{0}, \omega_{1}$ are the values of $\omega$ at the beginning and end of the interval $t$. Thus we learn that the changes in $\omega$ are very much greater than those in $e$, but that by the end of one revolution both have returned to their original values. 


\section{CHAPTER IV.}

THE MOTION OF FLUIDS, AND THE RESISTANCE TO BODIES MOVING IN THEM. SECTION VII.

1. Newton's investigation of the law of resistance to similar bodies.

2. The manner in which the resistance depends on the form of the body.

Newton, xxxiv. \& xxxv.

3. Their resistance when the body is a surface of revolution.

4. The surface of least resistance, its properties and form. Scholium, Prop. xxxiv.

5. The law of resistance deduced from experiment. Prop. xl. and Scholium.

1. Suppose we have two systems of an equal number of particles similarly placed, and proportional each to each both in density and volume. Let them begin to move, the particles of one system amongst themselves, and those of the other amongst themselves, with like motions and in proportional times. If no action ever took place between the particles, by the first law of motion the similarity between the systems will always exist. It is also clear that any collisions or reflexions among the particles will not affect this similarity of motion; if any collision occur in one system an exactly similar collision will occur in the other; similar changes of motion will be thereby produced in the two systems.

Next, suppose the particles attract or repel each other with accelerating forces, which are as the squares of the velocities directly and the diameters inversely of the corresponding particles in the two systems. Consider two homologous particles, one in each system, the attractions 
of the rest on these being each in the above ratio, the resultants will also be in the same ratio, and the attracting particles at the beginning of the motion being similarly placed, and the forces in each system proportional, the directions of the resultants will be parallel. Now we know that two similar particles beginning to move in parallel directions will describe similar orbits in proportional times, when at the end of those times the directions of the forces are parallel and proportional to the squares of the velocities and the reciprocals of any homologous sides of their orbits. Hence these two particles begin to move similarly under the action of such forces as tend to preserve the similarity of their motions. And the same is true for all homologous particles in the two systems. Hence all the particles of the one system at the end of any small time $\tau$, are placed similar to those of the other at the end of the small proportional time $\tau^{\prime}$ and are moving in a similar manner. Hence the same thing will again be true at the end of the next proportional intervals, that is, at the end of the proportional times $2 \tau$ and $2 \tau^{\prime}$. Therefore the particles will continue always to move among themselves with like motions and in proportional times.

Let there be two fluids or systems such that the particles of the one are similar to those of the other; let the diameters and distances of any two particles in one system be $d$ times the diameters and distances of the corresponding particles in the other, and let the density of these particles in one system be $\rho$ times that of the corresponding ones in the other system. Let the particles begin to move from similar positions, and if we suppose the forces in the two systems to be always proportional to the squares of the velocities directly and the diameters of the corresponding particles inversely, the several particles will describe similar orbits in similar times. Let the velo- 
city of any particle in one system be $v$ times that of the corresponding particle in the other when at the corresponding part of its orbit. Let two large bodies which are similar to each other in the same manner that two corresponding particles are similar, be similarly projected into these two systems. They will then describe similar orbits in proportional times. The diameter of one body is $d$ times that of the other, and the velocity of one will be $v$ times that of the other. Let us consider the resistances to these bodies: it will arise partly from the centripetal forces with which the particles and the body act on each other, and partly from the collisions and reflexions of the particles and the body. The resistances of the first kind are, by hypothesis, as the squares of the velocities directly and the diameters of the corresponding particles inversely, and the masses of those particles directly, that is, the ratio of the resistances in the two systems is

$$
\frac{v^{2}}{d} \cdot d^{3} \rho=v^{2} \cdot d^{2} \cdot \rho .
$$

The resistances of the second kind are as the number of reflexions and the forces of those reflexions. The number of the reflexions in the two systems are as the velocities of the corresponding particles directly, and the spaces between their reflexions inversely, hence the ratio is $\frac{v}{d}$. The forces of the two systems are as the velocities and masses of the corresponding particles, hence their ratio is $v . d^{3} . \rho$; hence the ratio of the resistances is

$$
\frac{v}{d} \times v d^{3} \rho=v^{2} \cdot d^{2} \cdot \rho
$$

joining these two ratios, the ratio of the whole resistance in the two systems will be

$$
v^{2} \cdot d^{2} \cdot p \text {. }
$$

In such fluids, and under such conditions as those we 
have just been considering, the resistances vary as the square of the velocity, the square of the diameter, and the density of the fluid.

If we have two fluids whose particles when at a distance do not act with any force on each other, such fluids come under the description of the similar systems just considered. Let the particles of the two fluids be equal, then the resistances to equal similar bodies moving in them are accurately as the squares of the velocities of the bodies and the densities of the fluids. Next, suppose the bodies not equal. Because the motion of the fluid varies continuously from point to point, and because the force of collision due to two equal particles moving in the same manner is equal to that of one particle of double size, the forces of collision will be the same if we divided the fluid into elements, and considered them as particles. Let the equal fluids be divided into elements, which are proportional to the volumes of the similar bodies moving in them. Then the resistances will vary as the square of the diameter, the square of the velocity of the body, and the density of the fluid.

But how far are we justified in applying these conclusions to the fluids we meet with in nature? The forces to which collision and reflexion are due, are those which are sensible only at distances which are indefinitely small compared with the average uistances between the particles. Are these the only forces which exist between the particles of a fluid? Incompressible fluids are the nearest approach to such a state of things. In elastic fluids the particles have a tendency to recede from each other, and our previous reasoning cannot therefore apply to them.

Let there be three fluids A, B, C; let them consist of similar and equal particles regularly disposed at equal distances, and let the parts of $\mathrm{A}$ and $\mathrm{B}$ have a tendency to 
recede from each other with forces that are as $\mathrm{T}$ and $\mathrm{V}$, and let the particles of the medium $\mathrm{C}$ be entirely destitute of such forces. Let four equal bodies move in these media, viz.

$\mathrm{D}$ in the medium $[\mathrm{A}$,$] and \mathrm{E}$ in $[\mathrm{B}]$
$\mathrm{F}$ and $\mathrm{G}$ in $[\mathrm{C}]$

and let

$$
\frac{\text { vel. of } \mathrm{D}}{\text { vel. of } \mathrm{E}}=\frac{\text { vel. of } \mathrm{F}}{\text { vel. of } \mathrm{G}}=\sqrt{\frac{\mathrm{T}}{\mathrm{V}}} \text {; }
$$

then since the forces are as the squares of the velocities, and the diameters of the particles are equal, therefore the resistances in the two fluids are as the squares of the velocities, that is

$$
\frac{\text { Res. to } \mathrm{D}}{\text { Res. to } \mathrm{E}}=\frac{\text { Res. to } \mathrm{F}}{\text { Res. to } \mathrm{G}}=\frac{\mathrm{T}}{\mathrm{V}} \quad-
$$

Let us suppose also that

$$
\begin{aligned}
\text { vel. of } D & =\text { vel. of } F \\
\therefore \text { vel. of } E & =\text { vel. of } G
\end{aligned}
$$

augment the velocities of $\mathrm{D}$ and $\mathrm{F}$ in any ratio, and diminish the force $\mathrm{V}$ of the particles in the medium $\mathrm{B}$ in the duplicate of that ratio, the medium $\mathrm{B}$ will approach to the form and condition of the medium $\mathrm{C}$, and therefore the resistances to the equal and equally swift bodies $\mathrm{E}$ and $\mathrm{G}$ moving in those media will approach equality. Hence by (2.) the bodies $\mathrm{D}$ and $\mathrm{F}$, when they move with great swiftness, meet with resistances nearly equal. Hence the resistance to a body moving very swiftly in an elastic fluid is almost the same as if the parts of the fluid were destitute of their centrifugal forces and did not tend to fly from each other. So that the resistance to similar bodies moving very swiftly in an elastic medium vary as the squares of the velocities and the squares of the diameters. 
This reasoning requires that the velocity should be so great that the forces of the particles will not have time to act.

2. The preceding investigation has led us, on certain assumptions, to the law of resistance to similar bodies, but it now remains to discover what change in the resistance would be caused by a change of form in the body. A new assumption becomes necessary. Let us suppose the particles to be so rare that their distances are infinitely greater than their diameters, so that each particle may be able to give its blow to the body and then to make its escape without affecting the particles which have not yet given their stroke. It is manifest that to find the resistance according to this principle we have to divide the surface into elements, find the resistance on those elementary planes separately, and by integration add the results. It is necessary to find the resistance on a small plane inclined at an angle $\theta$ to the direction of motion. Let the area of the plane be $A$, then the number of particles that will strike it will be proportional to $A \cos . \theta$ and to the velocity $v$ conjointly, and each particle will strike the plane with a normal velocity $v \cos . \theta$. The mass of each particle is supposed the same. Hence the whole normal resistance will be proportional to $\left.\mathrm{A} \overline{\cos _{.} \theta}\right|^{2} v^{2}$, and resolving this along the direction of motion the resistance will be proportional to $\overline{\mathrm{A} \cos . \theta^{3}} v^{2}$. Hence if $\varkappa \mathrm{A} v^{2}$ be the resistance on the plane when perpendicular to the direction of motion, the resistance when inclined at an angle $\theta$ will be

$$
\left.\varkappa \mathrm{A} v^{2} \overline{\cos . \theta}\right|^{3} \text {. }
$$

It will be observed that this reasoning is true whether the particles be elastic or not. Any change of elasticity affects the resistance by changing $x$.

Let a cylinder be made to adrance in the direction of its 
axis with a uniform velocity $v$ in a medium, and let us suppose that the particles of the fluid are perfectly elastic. They will then rebound with the same velocity relatively to the cylinder as that with which they struck it. Therefore the cylinder, on striking each particle, givesit a velocity twice its own, and in moving forwards a length half its axis communicates a motion to the particles which is to the whole motion of the cylinder as the density of the medium to the density of the cylinder. Hence the cylinder meets a resistance which is to the force by which its whole motion may be taken away in the time in which it describes half its axis as the density of the medium is to the density of the cylinder. If $l$ be the length of the axis, the time of describing the half axis will be $\frac{1}{2} \frac{l}{v}$, and the accelerating force that would generate a velocity $v$ in this time is $\frac{2 v^{2}}{l}$; hence the moving force, which is the resistance, is

$$
2 \mathrm{~A} v^{2} \rho,
$$

where $A$ is the area of the base, and $\rho$ the density of the fluid.

Next, let us suppose the particles perfectly inelastic; they will not be reflected, and the cylinder will merely communicate its own simple velocity to the particles it strikes against. The resistance is therefore only half as great as before, that is

$$
\text { Resistance }=\mathrm{A} v^{2} \rho \text {. }
$$

Thirdly. If the particles be imperfectly elastic, the particles will rebound from the cylinder with a less velocity than if they were elastic, and a greater velocity than if they were inelastic; hence

$$
\text { Resistance }=x \mathrm{~A} v^{2} \rho,
$$

where $x$ is some quantity lying between 1 and 2 . 
3. Let us now apply this to find the resistance on a surface of revolution moving in the direction of its axis. Let $v$ be the velocity of the body relative to the fluid. We shall then suppose the fluid in front of the body to be at rest. Take the axis of revolution of the surface as the axis of $x$, let $y$ be the ordinate and $s$ the arc of the generating curve. By what has been already said, the resistance or pressure on the annulus $2 \pi y d s$ when resolved along the axis will be

$$
2 \pi y d s \cdot x v^{2} \cdot\left(\frac{d y}{d s}\right)^{3},
$$

because this latter factor expresses the cosine of the angle the normal makes with the line of motion of the body. Hence the whole resistance will be

$$
2 \pi \times v^{2} \int y\left(\frac{d y}{d s}\right)^{2} d y \text {. }
$$

Let the surface be terminated by a plane perpendicular to the axis of $x$, the section will be a circle; let $r$ be its radius. Let $y=\eta \cdot r$, and $s=\sigma \cdot r$, then the whole resistance will be

$$
2 \pi x v^{2} \cdot r^{2} \int \eta\left(\frac{d \eta}{d \sigma}\right)^{2} d \eta \text {. }
$$

Thus, assuming merely that the distances of the particles are infinitely greater than their diameters, we learn that in the same fluid the resistance varies as the square of the velocity, and in similar bodies as the square of their radii.

It is not necessary that all the particles should be of equal size, or at exactly equal distances. It is sufficient that the equal particles be equally scattered in vast numbers throughout the fluid. For as the distances of the particles are supposed indefinitely small, so vast a number of particles strike the surface that it will be sufficient to consider only their average size and distance. 
If the particles of a fluid become packed closer together, so that the fluid becomes denser in the ratio of $\rho$ to 1 , it is manifest that the number of particles striking the surface will be increased in this ratio, and therefore the resistance will be increased in the ratio of $\rho$ to 1 . If the masses of the particles be increased in the ratio $\rho$ to 1 , the density of the fluid will be increased in this ratio, the force of each impact will be increased in this ratio, and the resistance will be increased in the ratio of $\rho$ to 1 . Hence the resistance varies as the density of the fluid.

To show that the resistance on a sphere is half that on one of its great circles. Let $r$ be the radius of the sphere, $\theta$ the angle any radius makes with the direction of the motion. Then, by the general rule, the resistance on the annulus $2 \pi r \sin . \theta \cdot r d \theta$ will be

$$
2 \pi r^{2} \sin . \theta d \theta \overline{\cos \cdot \theta_{j}^{3}} \cdot x v^{2} \text {. }
$$

Hence the whole resistance will be

$$
\begin{gathered}
2 \pi \times v^{2} r^{2} \int \overline{\cos .} \theta \mid 3 \sin \theta d \theta \\
=2 \pi \times v^{2} r^{2} \cdot \frac{r}{4},
\end{gathered}
$$

the limits of integration being from $\theta=0$ to $\theta=\frac{\pi}{2}$. But the resistance on a circle of radius $r$ is

$$
x v^{2} \cdot \pi r^{2} \text {, }
$$

which is just double the former result.

These results were afterwards modified by Newton. A course of reasoning, which we shall consider in another chapter, led him to the conclusion that the resistance on a sphere is equal to $\frac{1}{4} c \rho v^{2}$, where $c$ is the area of one of its great circles.

4. Upon a circular base rad. $r$, construct a frustrum of a cone of given height $h$, such that the resistance on it may be 
less than that on any other frustrum of the same height and base, the motion being in the direction of the axis.

Produce the frustrum to its vertex, and let $2 \theta$ be the angle of the cone. Then it is evident that the radius of one end being $r$, that of the other will be $r-h$ tan. $\theta$. And the resistances on the curved and flat ends will be respectively

and

$$
\left.\sin ^{2} \theta\left(r^{2}-\overline{r-h \tan . \theta}\right)^{2}\right)
$$

the common constant factor $\pi x v^{2}$ being omitted. The sum of these two is easily seen to be

$$
r^{2}-r h \sin .2 \theta+h^{2} \sin ^{2} \theta \text {; }
$$

differentiating this we have

$$
h \sin . \theta \cos . \theta=r-2 r \sin ^{2} \theta \text {. }
$$

Put $x$ for the whole height of the cone, then will

$$
\begin{gathered}
h \cdot \frac{x \cdot r}{r^{2}+x^{2}}=r-2 r \cdot \frac{r^{2}}{r^{2}+x^{2}} \\
\therefore x^{2}-h x=r^{2} \\
\therefore x=\frac{h}{2}+\sqrt{r^{2}+\frac{h^{2}}{4}},
\end{gathered}
$$

whence this construction. Let $\mathrm{O}$ and $\mathrm{D}$ be the given centres of the two ends, and $C$ be any point in the circumference of the base. Bisect $\mathrm{OD}$ in $\mathrm{Q}$ and produce $\mathrm{QD}$ to $\mathrm{S}$, so that $\mathrm{QS}=\mathrm{Q} \mathrm{C}$. $\mathrm{S}$ is the vertex of the required cone. This result Newton thought might be of use in the building of ships.

To find the surface of revolution such that, when altogether immersed, the resistance on it will be less than on any other surface of the same length and breadth.

Take the axis of revolution as the axis of $x$, and let $y$ be the ordinate of the generating curve. And let $p$ be the diff. co. of $y$ with respect to $x$. Then, omitting con- 
stant factors, the resistance on the curve will be proportional to

$$
\int y \frac{p^{3}}{1+p^{2}} d x
$$

Then the general equation given by the Calculus of Variations leads at once to

$$
y=c \cdot \frac{\left(1+p^{2}\right)^{2}}{p^{3}}
$$

this, therefore, is the differential equation to the curve which generates the surface of least resistance. The construction which Newton gives agrees exactly with the above equation.

This equation cannot be integrated; we cannot, therefore, find the equation to the curve. If we could, we should have two constants in it, which are to be determined by the conditions, first, that when $x=o, y=a$, and when $x=l, y=b$, where $l$ is the length of the solid, $a$ and $b$ the radii of the bounding sections.

The equation to the surface of least resistance may be put under a more convenient form. Differentiating it we have

$$
\begin{aligned}
& d y=p d x=c \frac{p^{4}-2 p^{2}-3}{p^{4}} d p \\
& \therefore d x=c\left(\frac{1}{p}-\frac{2}{p^{3}}-\frac{3}{p^{5}}\right) d p .
\end{aligned}
$$

Hence,

$$
\left.\begin{array}{c}
a+\frac{x}{c}=\log \cdot p+\frac{1}{p^{2}}+\frac{3}{4 p^{4}} \\
y=c \cdot \frac{\left(1+p^{2}\right)^{2}}{p^{3}}
\end{array}\right\} .
$$

By eliminating $p$ the equation to the generating curve may be found.

If we wish to find where the curve cuts the axis of $x$, we have merely to put $y=o$; this leads to

$$
\text { Q } 4
$$




$$
c \frac{\left(1+p^{2}\right)^{2}}{p^{3}}=o ;
$$

we cannot have $c=o$, for then $y$ would be always nothing. The numerator of the fraction cannot be nothing, for it is always greater than unity. The denominator cannot become infinite without making the whole fraction infinite. Hence the curve never cuts the axis of $x$; the surface of . least resistance has a flat surface exposed to the resistance of the fluid. The next question naturally is, what is the least value of $y$ ? To find this we must put

$$
\frac{d y}{d p}=o \text {; }
$$

this gives $p= \pm \sqrt{3}$ and $\therefore y= \pm \frac{16}{3 \sqrt{3}} c$. At this point of the curve there must manifestly be a cusp.

As $p$ increases from zero, $y$ decreases from infinity; the curve approaches the axis. The tangent at the cusp lies between the two branches, one going off to $p=o$, the other to $p=\infty$. There are no asymptotes. If $y$ be positive, $p$ is positive. The theory of Equations shows that the equation

$$
p^{4}-p^{3} \frac{y}{c}+2 p^{2}+1=o,
$$

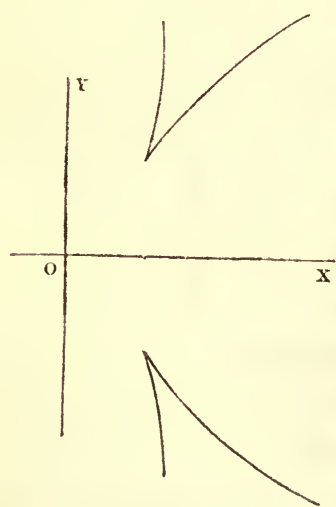

not having more than two changes of sign, cannot have more than two positive roots. Therefore the above two branches contain all that is above the axis of $x$. If we change the sign of $y$, we change the sign of $p$; hence the curve is the same on both sides of the axis of $x$. If we change the sign of $c$, we shall manifestly have the same curve, except that it is turned the other way, and lies on the opposite side of the axis of $y$. 
5. In order to investigate the resistances of fluids by experiment, Newton procured a square wooden vessel whose length and breadth on the inside was nine inches, English measure, and depth nine feet and a half. This he filled with rain-water, and having provided globes made of wax, with lead inclosed therein, he observed the times of the descents of these globes, the height through which they descended being 112 inches.

The resistance being supposed to vary as the square of the velocity, the height $x$, fallen in any time $t$, is given by

$$
x=u t-\frac{u^{2}}{g^{\prime}} \log .2+\frac{u^{2}}{g^{\prime}} \log \cdot\left(1+\varsigma^{-\frac{2 g^{\prime}}{u} t}\right)
$$

where $u$ is the "terminal velocity," and $g$ ' the relative force of gravity. It is necessary to find $u$. Let $\mathrm{A}$ be the weight of the globe in vacuo, $\mathrm{B}$ its weight in the resisting medium. Then the density of the medium is

$$
\frac{\mathrm{A}-\mathrm{B}}{\frac{4}{3} \pi a^{3} g},
$$

where $a$ is the radius of the globe, and $g$ the force of gravity; and therefore the resistance to the globe when moving with velocity $u$, is

$$
\frac{3}{16} \cdot \frac{\mathrm{A}-\mathrm{B}}{a g} \cdot u^{2},
$$

and this is to be equal to the weight of the body, which is $B$; therefore

$$
\left.\begin{array}{l}
u^{2}=\frac{16}{3} \cdot \frac{\mathrm{B}}{\mathrm{A}-\mathrm{B}} \cdot a g \\
g^{\prime}=\frac{\mathrm{B}}{\mathrm{A}} \cdot g .
\end{array}\right\} .
$$

The value of $\mathrm{B}$ can be found by weighing the globe in water. To find A we must weigh it in air, suppose we 
have W. According to Newton the density of water is 860 times that of air; hence

$$
\begin{aligned}
& A-B=860(A-W) \\
& \therefore A=W+\frac{W-B}{859},
\end{aligned}
$$

and

$$
A-B=(W-B)\left(1+\frac{1}{859}\right) .
$$

The value of $a$ can be found from those of $\mathrm{A}$ and $\mathrm{B}$, for the weight of a globe of water one inch in diameter is 132.645 grains in air, and therefore 132.8 grains in vacuo. And since the weights of globes vary as the cubes of their diameters, hence

$$
8 a^{3}=\frac{\mathrm{A}-\mathrm{B}}{132.8},
$$

where $\mathrm{A}-\mathrm{B}$ is measured in grains, and $a$ in inches.

We are now therefore able to calculate the value of $x$ when $t$ is given. If $t$, be not very small, the last term may be neglected. For since when $\alpha$ is small

$$
\log .(1+a)=a \text { nearly, }
$$

the value of that term is very nearly

$$
\frac{u^{2}}{g} \cdot \varepsilon^{-\frac{2 g^{\prime} t}{u} t},
$$

and the exponent being large, this term will be insensible. We may therefore take

$$
x=u t-\frac{u^{2}}{g^{\prime}} \log .2 .
$$

The space deduced from this formula requires a correction depending on the narrowness of the wooden ressel in which the experiments were made. Now if a globe descend in a cylinder, the area of whose section is $\mathrm{L}$, the resistance will be increased in the ratio 


$$
\frac{\mathrm{L}}{\mathrm{L}-\frac{1}{2} \pi a^{2}} \cdot\left(\frac{\mathrm{L}}{\mathrm{L}-\pi a^{2}}\right)^{2}=\mu^{2} \text {. }
$$

Hence $u^{2}$ will be decreased in the same ratio, and $\therefore$ $u$ decreased in the ratio of $\mu: 1$. In all the following experiments the area of the greatest circle of the sphere is small compared with the section of the cylinder; the value of $\mu$ is therefore nearly unity. In the expression for $x$, the first term is usually a hundred times the second; hence it will be sufficiently accurate to reduce the space $x$ by simply multiplying it by $\mu$. This will be evident on an inspection of the numbers in the following experiments.

Experiment 1. A globe whose weight was $156 \frac{1}{4}$ grains in air, and 77 grains in water, described the whole height of 112 inches in 4 seconds. On repeating the experiment, the globe again spent the very same time of 4 seconds in falling.

Here $\mathrm{A}=156 \frac{1}{3} \frac{3}{8}$ grains, $2 a=.84224$ inches,

$\therefore u=29.0311$ inches per second.

The principal term in $x$ is $116.1245,7$,

The second

, is 3.0676,$\}$

$$
\begin{aligned}
& \therefore x=113.0569 \text {, } \\
& \mu=.9914 \text {. }
\end{aligned}
$$

Hence the space which the globe falling in water describes in four seconds is, by

$$
\begin{array}{ll}
\text { theory } & - \\
\text { experiment - } & -112.08,
\end{array}
$$

Experiment 2. Three equal globes, whose weights were severally $76 \frac{1}{3}$ grains in air, and $5 \frac{1}{16}$ grains in water, were let fall successively. Every one fell through the water in 15 seconds of time, describing in its fall 112 inches.

Here $\mathrm{A}=76 \frac{5}{1 \frac{2}{2}}$ grains, $2 a=.81296$ inches,

$$
\therefore u=7.712 \text { inches per second. }
$$


The principal term in $x$ is 115.678 ,

The second

is 1.609 ,

$$
\therefore x=114.069 \text {, }
$$

and

$$
\mu=\quad .895 \text {. }
$$

Hence the space which the globe falling in water describes is, by

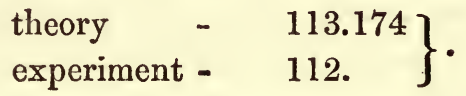

Experiment 3. Three equal globes, whose weights were severally 121 grains in air and 1 grain in water, were successively let fall, and they fell in water through a height of 112 inches in the times $46^{\prime \prime}, 47^{\prime \prime}$, and $50^{\prime \prime}$. By theory these globes ought to have fallen in about $40^{\prime \prime}$. The weight of the globe in water is so small, that any errors in the weighing, or produced by the rarefaction of the wax, by the heat of the hand, or by the weather, or by bubbles of air adhering to the globes, become sensible. That the experiment may be certain, the weight of the globe in water should be several grains.

Newton began the foregoing experiments to investigate the resistances of fluids, before he had discovered the theory laid down in the preceding sections. In order to examine the theory after its discovery, he undertook another course of experiments. He procured a wooden vessel, whose breadth on the inside was $8 \frac{2}{3}$ inches and depth $15 \frac{1}{3}$ feet. He made four globes of wax with enclosed lead, each weighing $139 \frac{1}{4}$ grains in air, and $7 \frac{1}{8}$ grains in water. These he let fall, measuring the times of their falling in the water with a pendulum oscillating half seconds. The globes were cold, and had remained so for some time, both when they were weighed and when they were let fall, because warmth rarefies the wax, and by rarefying it diminishes the weight of the globe in water, 
and wax when rarefied is not instantly reduced by cold to its former density. Before they were let fall, they were totally immersed in water, lest, by the weight of any part of them that might chance to be above water, their descent should be accelerated in the beginning. Then, when after their immersion they were perfectly at rest, they were let go with the greatest caution, that they might not receive any impulse from the hand that let them down. At Newton's first experiments the weather was a little colder than when the globes were weighed, and therefore he repeated the experiments another day. On making the experiments several times over, he found that the globes fell mostly in the times of $49 \frac{1}{2}$ to 50 oscillations, the times varying, however, from $47 \frac{1}{2}$ to 53 oscillations. On computing from the theory the time of descent, it was found to be 50 oscillations, very nearly. Newton tried eight other sets of experiments, in all of which there was a tolerable accordance between theory and experiment. The times in which the balls descended varied, sometimes as much as one-fifth of the whole time of descent; but the errors were generally as much on one side as on the other of the theoretical result. Whence, for such swift motions as these, we may conclude that the resistance on a globe is very nearly represented by the preceding theory. Those globes which fell with a slower motion were found to agree best with the theory, and the reason of this is as follows. When the globes were first let fall, it was found that they oscillated about the centre, that side which is heaviest tending to descend first. The globe in consequence of these oscillations communicated a greater motion to the fluid than it otherwise would, that is, it met with a greater resistance. In the heavier and larger globes this oscillation was not checked by the water until after several oscillations, and hence these were more resisted than the 
lighter ones, which fell slower. Every care was taken to diminish these oscillations, but they could not be altogether prevented.

From the top of St.Paul's, in June, 1710, two glass globes were allowed to fall through the height of 220 English feet. One was full of quicksilver, the other contained air. The two globes rested on a wooden table, which turned round iron hinges on one side, the other side being supported by a wooden pin. The two globes were let fall together by pulling out the pin by means of an iron wire reaching from thence quite down to the ground, so that the pin being removed, the table, which had then no support but the iron hinges, fell downwards, and turning round, the globes dropped off it. At the same instant with the same pull of the iron wire that took out the pin, a pendulum, oscillating seconds, was set in motion. The wooden table was not found to turn so quickly on its hinges as it ought to have done. Hence the times of descent were prolonged $3^{\prime \prime}$. This must be taken into account, as in that time the ball full of mercury would have described 37 feet. The difference between theory and experiment was found to be very small. To mention one instance, one globe of air fell 220 feet in 8.2"; according to theory it should have fallen in that time 225 feet 5 inches. The resistances to the globes of mercury were found to be so small in comparison with their weight, that they fell in nearly the same time which they would have taken to fall through the same height in vacuo. The differences could not be observed with sufficient accuracy to furnish a test of the theory, the difference being sensibly the same for globes of all weights.

In July, 1719, Dr. Desaguliers made some other experiments of the same kind. The globes let fall were formed of hogs' bladders blown full of air. They were 
let fall from the lantern at the top of the cupola of the same church, a height of 272 feet. The agreement between theory and experiment was even more striking than in the last experiments.

The theoretical results are calculated on the supposition that the resistance is proportional to the square of the velocity, the density of the fluid, and the surface of the sphere. The comparison with experiment serves to test all these laws. We may, therefore, conclude that for such velocities as those here experimented on, varying from 6 to 30 feet per second, and for spheres of radii varying from $\frac{1}{2}$ to 3 inches, and for the fluids of air and water, those three laws are tolerably correct.

Newton remarks that these experiments are more accurate than those he made with pendulums; for the vibrations not being very small, the body always excited a motion of the fluid contrary to the motion of the pendulum on its return, and thus the whole resistance appeared greater than it really was.

[See Note V.] 


\section{CHAPTER V.}

THE MOTION OF A PENDULUM.

1. Some general considerations.

2. Motion in vacuo, Newton xxiv.

3. The properties of a pendulum.

4. Motion in a resisting medium - Modern method of considering the perturbations of a pendulum, - the Newtonian method.

5. Newton's experiments to discover the law of resistance.

1. The importance of the pendulum can hardly be overrated. It ministers to our comforts in a variety of ways. But what is more to our present purpose, it is a powerful engine of discovery. It can be made to test the laws of impact, it teaches us the law of resistance by which fluids retard the motion of bodies moving in them. It enables us to note the variations of gravity over the earth, and thus reveals to us not only the form of the world, but also the force with which it attracts external objects. It has even been applied lately to prove ocularly the rotation of the earth. There is no end to its applications. It is the great accuracy with which the time of oscillation may be observed that renders this instrument so useful. By noting the time of any great number of oscillations, and dividing that time by the number of oscillations, we can find the time of any one with great accuracy. It is, therefore, highly necessary for us to consider carefully the properties of a pendulum.

A pendulum is any solid body which oscillates about a fixed horizontal axis. A simple pendulum consists of a 
material particle suspended from a fixed point by an inflexible inextensible string without weight. The length of this string is called the length of the simple pendulum.

A perfect simple pendulum is only a mathematical idea; we may approximate to such an instrument, but we cannot accurately construct it. It will, therefore, in many cases be necessary to have the means of determining, when any compound pendulum is given, the length of the equivalent simple pendulum. The first person who solved this generally was Huygens in his " Horologium Oscillatorium," 1673. The principle on which he proceeded was not so simple as that to which this and such like problems are now referred. But the result is that if $h$ be the distance of the centre of gravity from the axis of suspension, and $m i^{2}$ the moment of inertia about an axis through the centre of gravity parallel to the axis of suspension, the length of the equivalent simple pendulum will be

$$
l=\frac{i^{2}+h^{2}}{h} \text {. }
$$

This is on the supposition that the body moves in vacuo under the action of gravity. If it move in a medium resisting according to any function of the velocity, the above will still be the length of the equivalent simple pendulum. For the resistance on a unit of area being supposed to be $x v^{n}$, according to the usual theory of resistance, the moment of the whole resistance on the surface will be

$$
\text { A. } v^{n} \text {, }
$$

where A depends on the form of the surface, and not on the nature of the motion. Hence to make the simple pendulum move in the same manner, we have merely to suppose that the weight of the particle is equal to the weight of the pendulum, and that it experiences a resistance which 
follows for variations of velocity the same law that any element of the pendulum experiences, but whose magnitude is such that the whole moments of the resistance on the pendulum and particle about the axis of suspension are equal. In reasoning then on the pendulum we may always consider it as a simple pendulum. For when we have determined its motion, the preceding formulæ enable us to determine that of the compound pendulum.

Newton does not confine himself to the case in which the particle describes a circle. Supposing the string flexible, he has given in the first book a way of making it describe any given curve. Of all these the cycloid is that which possesses the most important properties. The motion of a particle in a cycloid is discussed, because it gives us a deeper insight into the laws of pendulous movements than that in any other curve. The motion of a particle in a circle is considered, because in practice most pendulums are so. constructed that any point in them describes a circle.

[See Note VI.]

A particle constrained to move in a cycloid whose axis is vertical is acted on by gravity and resisted by a constant force. To determine the motion. Newt. xxv.

Let $l$ be twice the radius of the generating circle, $s$ the distance of the particle at any time $t$ from the lowest point (C) of the cycloid, $v$ the velocity, and $m$ the mass of the particle. Let $f$ be the constant resistance.

Then the moving force along the tangent is

$$
-\frac{m g}{l} s+f
$$

supposing the particle to be descending. 
But this is also $m \frac{d^{2} s}{d t^{2}}$, hence we have

$$
\frac{d^{2} s}{d t^{2}}+\frac{g}{l} s=\frac{f}{m}
$$

This equation being of a standard form, we can write down its integral,

$$
s=\mathrm{L} \cos \sqrt{\frac{g}{l}}(t-\lambda)+\frac{l f}{m g}
$$

where $L$ and $\lambda$ are constants depending on the initial conditions of motion.

Take a point $\mathrm{O}$ in the arc, so that $\mathrm{CO}=\frac{l f}{m g}$, and let $s^{\prime}$ be the arc when measured from this point. Then

$$
s^{\prime}=\mathrm{L} \cos \sqrt{\frac{g}{l}}(t-\lambda) .
$$

Suppose the particle began its descent from $D$, then $\lambda$ is clearly the time at which the particle was at $\mathrm{D}$, and $\mathrm{L}=\operatorname{arc} \mathrm{OD}$. It is manifest the greatest velocity will be at $O$. If the particle had been undisturbed by $f$, the same equation would have given the arc measured from C.

If then the particle when resisted by $f$ be at $\mathrm{P}$ at any time, and if another particle not resisted by $f$ be at $Q$ at the same time, then $\mathrm{DP}$ bears to $\mathrm{PQ}$ the constant ratio DO to DC.

The effect of any constant resistance is to diminish the arc continually in an arithmetical progression, but not to affect the time of oscillation. 
$A$ particle constrained to move in a cycloid whose axis is vertical, is acted on by gravity and resisted by the medium in which it moves in the ratio of the velocity. To determine the motion. Newt. xxvi.

Let $l$ be twice the radius of the generating circle. Let $s$ be the distance of the particle at any time $t$ from the lowest point of the cycloid, and $v$ be the velocity, and $m$ the mass of the particle. Let $x$ be the coefficient of resistance. Then the moving force along the tangent will be

$$
-\frac{m g}{l} \cdot s-x v,
$$

the particle being supposed ascending its arc. But this is also $m \frac{d^{2} s}{d t^{2}}$. Hence

$$
\frac{d^{2} s}{d t^{2}}+\frac{x}{m} \cdot \frac{d s}{d t}+\frac{g}{l} s=0
$$

is the equation to find $s$ in terms of $t$.

To integrate this equation, put

$$
s=u \cdot \varepsilon^{\mu t}
$$

where $\mu$ is some constant at our disposal ; on substitution, the equation becomes

$$
\frac{d^{2} u}{d t^{2}}+\left(2 \mu+\frac{x}{m}\right) \frac{d u}{d t}+\left(\mu^{2}+\mu \frac{x}{m}+\frac{g}{l}\right) u=0 .
$$

Let us then choose $\mu$ so that

$$
2 \mu+\frac{x}{m}=0
$$

and the above equation is reduced to the standard form

$$
\begin{gathered}
\frac{d^{2} u}{d t^{2}}+\left(\frac{g}{l}-\frac{x^{2}}{4 m^{2}}\right) u=0 . \\
\text { Put } n^{2}=\frac{g}{l}, n^{\prime 2}=\frac{g}{l}-\frac{x^{2}}{4 m^{2}}
\end{gathered}
$$




$$
\therefore u=\mathrm{L} \cos n^{\prime}(t-\lambda) \text {. }
$$

Supposing the time to be counted from the epoch when the pendulum began its first complete oscillation from its highest point, then $\lambda=0$.

$$
\therefore s=\mathrm{L} \varepsilon^{-\frac{x}{2 m} t} \cos n^{\prime} t
$$

Hence we learn,

1. That the oscillations will be isochronal, and the time of oscillation

$$
=\frac{\pi}{n^{\prime}}=\frac{\pi}{\sqrt{\frac{g}{l}-\frac{x^{2}}{4 m^{2}}}}
$$

This differs from the time of oscillation in a vacuum; if $x$ be small, and $T$ the time in vacuo, the difference will be very nearly

$$
=\frac{x^{2} l}{8 m^{2} g} \text {. }
$$

If we neglect terms so small as this, the time will be unaltered.

2. The arcs of each successive oscillation will decrease in geometrical progression, and if $\mathrm{L}$ be any one such arc, the next will be $\mathrm{L} \varepsilon^{\frac{-g z}{m n^{\prime}}}$.

A particle is constrained to oscillate in a cycloid whose axis is vertical under the action of gravity, and is resisted by the medium in which it moves in the ratio of the square of the velocity. Determine the motion. Newt. xxix.

Following the same notation as before, it is manifest that the equation of motion will be

$$
v \frac{d v}{d s}=-n^{2} s-\frac{x}{m} v^{2}
$$


where $n^{2}=\frac{g}{l}$, and the particle is supposed to be moving in the direction in which $s$ is measured. When the particle moves in the opposite direction we must change the sign of $x$.

Although the above equation cannot be completely integrated in finite terms so as to find $s$ in terms of $t$, yet we can always find the velocity at any point of the arc. The equation can be put under the form

$$
\frac{d v^{2}}{d s}+2 \frac{x}{m} \cdot v^{2}=-2 n^{2} . s
$$

This is a standard form, and the integral will be

$$
\begin{aligned}
& v^{2} \varepsilon^{2} x^{\prime} s=-2 n^{2} \int s \cdot \varepsilon^{2 x^{\prime} s} d s \\
& =\mathrm{C}-\frac{n^{2}}{x^{\prime}} \cdot \varepsilon^{2 x^{\prime} s}\left(s-\frac{1}{2 x^{\prime}}\right)
\end{aligned}
$$

where $x^{\prime}$ has been put for $\frac{x}{m}$. We may find $\mathrm{C}$ either in terms of the whole arc described, or the velocity at the lowest point. The latter gives when $s=0, v=\mathrm{V}$, hence

$$
\begin{gathered}
\mathrm{C}=\mathrm{V}^{2}-\frac{n^{2}}{2 \varkappa^{\prime 2}} \\
\therefore v^{2}=\left(\mathrm{V}^{2}-\frac{n^{2}}{2{\chi^{\prime 2}}^{2}}\right) \varepsilon^{-2 x^{\prime} s}-\frac{n^{2}}{x^{\prime}}\left(s-\frac{1}{2 \kappa^{\prime}}\right)
\end{gathered}
$$

This finite expression will always give the velocity. As $x^{\prime}$ is usually small, it may be useful to expand the above in powers of $x^{\prime}$. We have

$$
\begin{aligned}
& v^{2}=\mathrm{V}^{2}-n^{2} s^{2}-\left(\mathrm{V}^{2}-\frac{2 n^{2} s^{2}}{2 \cdot 3}\right) 2 x^{\prime} s+\left(\mathrm{V}^{2}-\frac{2 n^{2} s^{2}}{3 \cdot 4}\right) \\
& \frac{\overline{\left.2 x^{\prime} s\right|^{2}}}{1.2}-\& \mathrm{c} . . . \overline{-1^{r}}\left(\mathrm{~V}^{2}-\frac{2 n^{2} s^{2}}{r+1}\right) \frac{\left.\overline{2 x^{\prime} s}\right|^{r}}{1.2 \ldots r}+\ldots
\end{aligned}
$$


Newton has given a geometrical construction for this velocity. But it is very long, and in the present age, when analysis is the great mathematical weapon, such a complicated construction is of no value except as a matter of curiosity.

If we wish to have the means of deducing the law of resistance from experiments on the pendulum, we must investigate the changes produced in the time and arc of vibration by a resistance that varies according to any law, indeed by any small disturbing cause whatever. The two next propositions of Newton have this for their object. They are entirely geometrical and the investigations too complicated to be inserted here. Of one of them Newton says: "by reason of the difficulty by which the resistance and velocity are found by this proposition we have thought fit to subjoin the following." We shall first discuss the modern analytical method* of determining the effect of any small disturbing cause, and then give an analytical proof of Newton's general proposition.

Let the quantities $s, t, n, \& \mathrm{c}$. have the same meaning as before, and let $f$ be the small disturbing cause which acts along the tangent on the particle.

The equation of motion will then be

$$
\frac{d^{2} s}{d t^{2}}+n^{2} s=f
$$

If $f=o$, the motion will be given by

$$
\left.\begin{array}{rl}
s & =a \sin (n t+b) \\
\frac{d s}{d t} & =a n \cos \cdot(n t+b)
\end{array}\right\}
$$


Assume these to be the true equations of motion when $f$ is not zero, then $a$ and $b$ are functions of $t$.

And since the second equation is the differential of the first,

$$
\sin .(n t+b) \frac{d a}{d t}+a \cos \cdot(n t+b) \frac{d b}{d t}=0 ;
$$

and since they satisfy the original equation of motion,

$$
n \cos .(n t+b) \frac{d a}{d t}-n a \sin \cdot(n t+b) \frac{d b}{d t}=f .
$$

Hence, solving these

$$
\left.\begin{array}{l}
\frac{d a}{d t}=\frac{f}{n} \cdot \cos \cdot(n t+b) \\
\frac{d b}{d t}=-\frac{f}{n a} \sin \cdot(n t+b)
\end{array}\right\}
$$

These equations, when solved, will give the changes in the arc and time produced by the cause $f$.

Suppose $f$ to be a small force. Then the variations of $a$ and $b$ are very small, and being multiplied on the right hand side by the small quantity $f$ we may neglect them. Hence if $a$, and $b$, be the altered values of $a$ and $b$,

$$
\begin{gathered}
a,-a=\frac{1}{n_{\mathrm{e}}} \int f \cos \cdot(n t+b) d t \\
b,-b=-\frac{1}{n a} \int f \sin \cdot(n t+b) d t .
\end{gathered}
$$

Hence we learn that if $f$ consist of two disturbing causes, the total disturbance will be nearly equal to the sum of the separate disturbances.

Suppose $f$ to be the resistance of a medium varying as the $m^{\text {th }}$ power of the velocity $f=x v^{m}$. The velocity in moving from the lowest point is 


$$
\begin{gathered}
v=a n \cos (n t+b) \\
\therefore f=x n^{m} a^{m} \cos ^{m}(n t+b)
\end{gathered}
$$

$\therefore$ substituting and performing the integration between the limits $n t+b=-\frac{\pi}{2}$ and $n t+b=\frac{\pi}{2}$ we have

$\left.\begin{array}{l}a,-a=-x a \cdot n^{m-2}: a^{m} \cdot \frac{m(m-2)(m-4) \& c .}{(m+1)(m-1)(m-3) \& c .} \\ b,-b=0,\end{array}\right\}$

where $a$ is $\pi$ or 2 according as $m$ is odd or even.

Hence when small quantities of the second order are neglected, the time of oscillation is unchanged and the arc continually decreases, and the difference between the arc described in the descent and that described in the subsequent ascent will be proportional to the same power of the arc that expresses the law of resistance with the velocity. This will enable us to find the law. Also if the difference of the arcs be represented by a series of terms such as

\section{$\mathrm{A} \mathrm{V}^{n}$}

where $\mathrm{V}$ is the velocity at the point where it is greatest, the resistance at this point will be represented by a series of terms such as

$$
\mathrm{M} \cdot \frac{g}{l} \cdot \alpha \cdot \frac{m(m-2) \cdots \cdots}{(m+1)(m-1) \cdots} \cdot \mathrm{AV}^{n},
$$

and the value of $\mathrm{V}$, the maximum velocity, can be always found by the formula

$$
\mathrm{V}=a \cdot \sqrt{\frac{\bar{g}}{l}}
$$

Also putting $\mathrm{R}$ for the moving force of the resistance, and W for the weight of the body, it is clear that

$$
a,-a \propto \frac{\mathrm{R}}{\mathrm{W}}
$$


the decrement of the arc varies inversely as the weight of the pendulum.

It will be inconvenient to constrain the particle to move in a cycloid: let us examine what errors would be introduced by making the particle vibrate in a circle.

Here the force is $g \sin \frac{s}{l}$ instead of $\frac{g}{l} s$, hence we must put

$$
\begin{gathered}
f=\frac{g}{l} s-g \sin \cdot \frac{s}{l}=\frac{g}{6 l^{3}} \cdot s^{3}, \text { nearly } \\
=\frac{g a^{3}}{6 l^{3}} \sin ^{3}(n t+b) .
\end{gathered}
$$

Substituting we have

$$
\left.\begin{array}{l}
a_{1}-a=0 \\
b_{1}-b=-\frac{g a \pi}{16 n l^{3}}
\end{array}\right\}
$$

the integrations being performed from $n t+b=o$ to $\pi$. Hence the arc is unaltered and the time increased by a quantity $\frac{b_{1}-b}{n \pi}=\frac{a^{2}}{16 l^{2}}$.

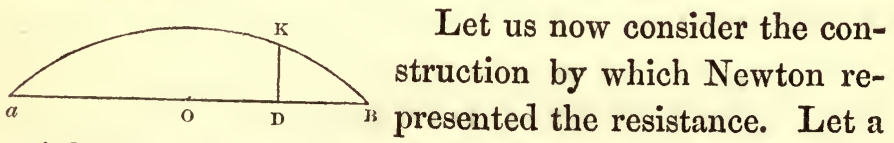
straight line $a \mathrm{~B}$ be drawn equal to the arc of the cycloid which an oscillating body describes, and at each of its points D draw the perpendicular D K equal to $\frac{1}{n^{2}}$ part of the resistance at $\mathrm{D}$, then $a_{0}$ and $a_{1}$ being the ares described in the descent and subsequent ascent, then

$$
\left(a_{1}-a_{0}\right) \frac{a_{1}+a_{0}}{2}=\text { area of curve } a \mathrm{~K} \mathrm{~B} \text {. }
$$


This admits of a very short proof, for the equation of motion being

$$
v \frac{d v}{d s}+n^{2} s=\mathrm{R},
$$

we have by one integration

$$
v^{2}+n^{2} s^{2}=\int \mathrm{R} d s
$$

and at the limits of integration $s=-a_{0}$ and $s=+a_{1}$, we have $v=o$

$$
\therefore n^{2}\left(a_{1}{ }^{2}-a_{0}{ }^{2}\right)=2 \int_{-a_{0}}^{a_{1}} \mathrm{R} d s,
$$

which is what we had to prove.

We have now to consider the nature of the curve $a \mathrm{~KB}$. We have accurately true

$$
\left.\begin{array}{l}
s=a \sin (n t+b) \\
v=a n \cos (n t+b)
\end{array}\right\}
$$

calling $y$ the ordinate and supposing the resistance to be equal to $k v^{m}$

$$
\therefore y=x a^{m} n^{m-2} \cos ^{m}(n t+b) \text {. }
$$

Let $x$ be the abscissa measured from the middle point $\mathrm{O}$ of $a \mathrm{~B}$, and

$$
x+\frac{a_{1}-a_{0}}{2}=a \sin (n t+b) .
$$

First, let the resistance vary as the velocity, then eliminating $t$,

$$
\frac{n y}{x}+\left(x+\frac{a_{1}-a_{0}}{2}\right)^{2}=a^{2} .
$$

If we neglect the variations of $a$ with $t$, this is the equation to an ellipse. The terms in $y$ thus neglected are of the order $x^{2}$. Secondly, let the resistance vary as the square of the velocity. Eliminating $t$ 


$$
\therefore \frac{y}{x}+\left(x+\frac{a_{1}-a_{0}}{2}\right)^{2}=a^{2} .
$$

If we neglect the variations of $a$ with $t$ this is the equation to a parabola. The terms in $y$ thus neglected are of the order $x^{2}$.

Newton takes these figures to be accurate enough for practical purposes, and we might now proceed to deduce the resistances at $\mathrm{O}$ from the difference of the arcs. But we have said enough to illustrate Newton's method. It is not so convenient for use as the more modern formula. But it is remarkable that the two methods are of equal degrees of approximation. Thus Newton arrived at as accurate a result as that which we now use, the only difference being that he expressed his result, according to the custom of the age, in a geometrical form.

We must now deduce the true law of resistance from a combination of theory and experiment. It is clear from what precedes, that the observations must be made on the decrements of the arcs. Newton suspended a wooden ball weighing $57 \frac{7}{2} \overline{2}$. ounces troy, its diameter being $6 \frac{7}{8}$ London inches, by a fine thread on a firm hook, so that the distance between the hook and the centre of oscillation of the globe was 10 feet. He marked on the thread a point 10 feet 1 inch distant from the centre of suspension, and even with this point he placed a ruler divided into inches, by the help whereof he observed the lengths of the arcs described by the pendulum. Then he numbered the oscillations in which the globe would lose $\frac{1}{8}$ part of its motion. In the following table the first column represents the first arc, or space the pendulum was drawn aside from the perpendicular; the second column the number of oscillations; the third column the last arc, which is always $\frac{1}{8}$ less than the first; and the fourth column the difierence between the first and last ares; 


$\begin{array}{rccc}2 & 164 & 1 \frac{3}{4} & \frac{1}{4} \\ 4 & 121 & 3 & \frac{1}{2} \\ 8 & 69 & 7 & 1 \\ 16 & 35 & 14 & 2 \\ 32 & 18 & 28 & 4 \\ 64 & 9 \frac{2}{3} & 56 & 8\end{array}$

The difference between the arc of descent and the subsequent arc of ascent will be above differences divided by the number of oscillations. In the greater arcs these are very nearly as the squares of the arcs described, and in lesser oscillations in a ratio somewhat greater. But theory taught us that if the resistance vary as the $m^{\text {th }}$ power of the velocity, the above difference of arcs should vary as the $m^{\text {th }}$ power of the arc. Hence the resistance when the globe moves very swift is in the duplicate ratio of the velocity nearly, and when it moves slowly, somewhat greater than in that ratio.

The points to be determined by experiment are how the resistance depends on the velocity, how on the surface of the body, and how on the density of the fluid. To determine the first, we must assume some law of resistance: let us suppose that the resistance varies partly as the velocity, partly as the square of the velocity, and partly in some intermediate ratio. This being the result suggested by the last-mentioned experiments; let the difference of the arcs be represented by

$$
\mathrm{A} \mathrm{V}+\mathrm{BV}^{\frac{3}{2}}+\mathrm{CV}^{2}
$$

where $\mathrm{V}$ is the greatest velocity in any oscillation. Then, according to the notation of the preceding theory, we have

$$
\mathrm{V}=\sqrt{\frac{g}{l}} a
$$

hence substituting, the difference of the axes will be represented by 


$$
\mathrm{A}^{\prime} \cdot \overline{2 a}+\left.\mathrm{B}^{\prime} \cdot \overline{2 a}\right|^{\frac{3}{2}}+\mathrm{C}^{\overline{2} a a^{2}}
$$

a form well adapted for a comparison with the results of experiments. Taking the second, fourth, and sixth experiments of the set discussed above, we get three equations to determine the three quantities $\mathrm{A}^{\prime}, \mathrm{B}^{\prime}, \mathrm{C}^{\prime}$.

$$
\begin{aligned}
& \mathrm{A}^{\prime}=.0000916, \\
& \mathrm{~B}^{\prime}=.0010847, \\
& \mathrm{C}^{\prime}=.0029588 .
\end{aligned}
$$

But the resistance will be expressed by

$$
\begin{gathered}
\frac{\mathrm{W}}{l}\left\{\frac{7}{11} \mathrm{~A}^{\prime} \alpha^{\prime}+\frac{7}{10} \mathrm{~B}^{\prime} a^{\prime \frac{3}{2}}+\frac{3}{4} c^{\prime} a^{\prime 2}\right\} \\
=\frac{\mathrm{W}}{l} \cdot\left\{.0000583 \alpha^{\prime}+.0007593 \alpha^{\prime \frac{3}{2}}+.0022169 a^{\prime 2}\right\}
\end{gathered}
$$

where $\mathrm{W}$ is the weight of the body. Thus for such swift motions in air as those varying from 4 to 120 inches per second, the resistance varies as the square of the velocity. Since $a^{\prime}$ in the second case represents 1 , in the fourth 4, in the sixth 16, the resistance will be to the weight of the globe, in the second case, as .0030345 to 121 , in the fourth .041748 to 121 , in the sixth .61705 to 121 .

The next point to be determined is the manner in which the resistance depends on the surface. For this purpose Newton suspended a leaden ball of 2 inches diameter, weighing $26 \frac{1}{4}$ ounces troy, by the same thread that he suspended the former ball, the length of the simple pendulum being $10 \frac{1}{2}$ feet. He found the resistance to the ball was $7 \frac{1}{3}$ times that on the former ball. But the ratio of the squares of the diameters was $11 \frac{1}{1} \frac{3}{6}$ to 1 nearly. "Therefore the resistance of these equally swift balls was in less than a duplicate ratio of the diameters. But the resistance of the thread has not yet been considered, which was certainly considerable. This could not be accurately 
determined, but it was found to be greater than a third part of the whole resistance of the lesser pendulum. When this third part is subtracted, the ratio $7 \frac{1}{3}$ to 1 becomes 7 to $\frac{2}{3}$ or $10 \frac{1}{2}$ to 1 , a ratio not very different from $11 \frac{13}{16}$ to 1 ." Since the resistance of the thread is of less moment in greater globes, Newton also tried the experiment with a globe whose diameter was $18 \frac{3}{4}$ inches. $\mathrm{He}$ found the resistance on this globe to be 7 times that on the first globe, whose diameter was $6 \frac{7}{8}$ inches; but the squares of the diameters are in the ratio of 7.438 to 1. The difference of these ratios is scarcely greater than what might arise from the resistance of the thread. Therefore the parts of the resistances which are in swift motions when the globes are equal as the squares of the velocities, are also when the velocities are equal as the squares of the diameters of the globes. We shall find occasion to modify this conclusion in another chapter.

In order to determine the manner in which the resistance depended on the density of the fluid, Newton calculated the resistance made to a body oscillating in water and in air, and found that that part which is proportional to the square of the velocity (and which alone it is necessary to consider in swift motions) is proportional to the density of the medium. "This is not perfectly accurate; for more tenacious fluids of equal density will undoubtedly resist more than those that are more liquid, as cold oil more than warm, warm oil more than rain water, and water more than spirits of wine. But in liquids which are fluid enough to retain for some time the motion impressed upon them by an agitation of the vessel, and which being poured out are easily resolvable into drops, the rule will be pretty accurate, especially for large bodies moving with a swift velocity.

"Lastly, since it is the opinion of some that there is a 
certain xtherial medium extremely rare, which freely pervades the pores of all bodies, and from such a medium some resistance must needs arise, in order to try whether the resistance which we experience in bodies in motion be made upon their outward superficies only, or whether their internal parts meet with any considerable resistance upon their superficies, I thought of the following experiment." Newton suspended a round deal box by a thread 11 feet long, and compared the resistance made to it when empty and when filled with lead. When full of air its weight was $\frac{1}{78}$ th of the weight when filled with metal. But since the decrement of the arc varies inversely as the weight, the box when filled with lead should make 78 oscillations before the arc was decreased by the same quantity that when filled with air it lost in one oscillation. But Newton counted 77 oscillations. Assuming that the greater resistance of the full box arises not from any other latent cause, but only from the action of some subtile fluid on the included metal, we may suppose that this resistance in equally swift bodies will be as the number of particles that are resisted. Let $A$ be the resistance on the external and $B$ on the internal superficies of the box when empty. Then $A$ and $78 B$ will be these resistances when the box is full. By the preceding experiment the resistance on the full box $A+78 B$ is to the resistance on the empty box $A B$ as 78 to 77 . Solving this simple equation, it follows that the resistance on the internal parts of the empty box $(B)$ will be $\frac{1}{59} \frac{2}{8}$ th part of the resistance $(A)$ on the external superficies. 


\section{CHAPTER VI.}

MOTION OF FLUIDS RUNNTYG OUT OF SMALL ORIFICES.

1. Newton's solution of the question, without limiting the orifice.

2. Newton's corollary - to deduce the resistance made by a fluid to a body moving in it.

3. The fallacy of Newton's reasoning - How others attempted to pursue the investigation. Note vii.

4. The velocity as given by the Equations of Motion. Note vii.

5. The efflux of an elastic fluid through a small orifice - strange conclusions to be deduced from the formula - St. Venant and Wantzels' experiments. Note vii.

1. THE problem to determine the motion of water running out of any vessel through a hole is so difficult that it has not yet been completely solved. We cannot therefore be surprised if Newton's solution of the question is not very satisfactory. He begins by considering a case in which he is able to give some account of the motion. Let us suppose that we have a circular cylinder filled with fluid, the surface of which is retained at a constant level in such a manner that the velocity of descent of all its parts is also uniform. In order to aid our conceptions, Newton supposes this effected by the descent of a cylinder of ice A PQB, of the same breadth with the cavity of the vessel, and having the same axis. The motion is supposed quite uniform, and its parts, as soon as they touch the superficies A B, are supposed to dissolve into water and flow down by their weight into the vessel. Let $u$ be the uniform velocity of descent of the cylinder, and let $k$ be the 
height through which a body must fall to acquire this velocity,

$$
\therefore u^{2}=2 g k \text {. }
$$

Let there be a hole $\mathrm{E} \mathrm{F}$ in the centre of the bottom, and let the whole cavity of the vessel which encompasses the falling water be full of ice, so that the water may pass through the ice as through a funnel. The particles of water are supposed to cohere a little, that in falling they may approach each other, and thus instead of being divided into several, they will form a single cataract. We

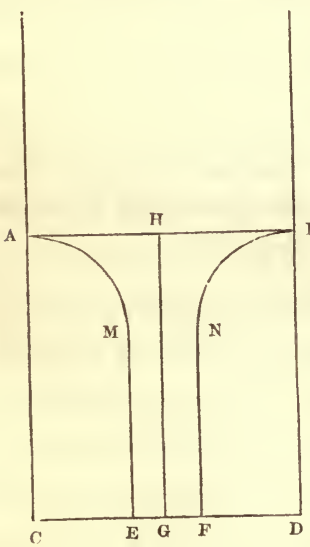
shall also suppose the form of the funnel such that the particles of water fall freely down. The lines of motion will form surfaces of revolution whose axis is the axis of the funnel, and the forces of cohesion acting on any particle have a resultant normal to these surfaces. The velocity of the particle will not therefore be affected by this force, and if $z$ be the depth of any particle below the plane $\mathrm{A} \mathrm{B}$ and $v$ its velocity we have

$$
v^{2}=u^{2}+2 g z ;
$$

each particle arrives with the same velocity at the aperture EF. The form of the funnel was supposed such as to admit of this motion; hence the area of any section must be inversely as the mean vertical velocity of the fluid for all parts of that section.

Now, if the particles of water did not exert any action on the icy funnel through which it flows we might suppose this funnel dissolved into water without changing the circumstances of the motion. But the descending fluid does act on the dissolved ice, and the whole nature of the motion is changed. But Newton argues that nevertheless the 
efflux of water, as to its velocity, will remain the same as before. It will not be less, because the ice now dissolved will endeavour to descend; it will not be greater, because the ice now water cannot descend without hindering that of other water equal to its own descent. The same force ought always to generate the same velocity in the effluent water. Newton does not mean that the same quantity of water flows out, but that the same velocity is generated. This is not, however, the vertical velocity at the orifice; " for the particles of water do not all of them pass through the hole perpendicularly, but flowing down on all parts from the sides of the vessel and converging towards the hole, pass through it with oblique motions, and in tending downwards meet in a stream whose diameter is a little smaller below the hole than at the hole itself." The particles of water at the actual orifice are not all moving in the same direction. Those in the centre are descending vertically while those near the circumference have a lateral motion, but at the vena contracta the whole fluid is descending vertically. If therefore $\mathrm{B}, \mathrm{B}^{\prime}$ be the areas of the sections of the vena contracta and orifice, and $v$ the velocity of the fluid at the vena contracta, the mean velocity of the fluid perpendicular to the orifice will be clearly

$$
\overline{\mathrm{B}} \overline{\mathrm{B}}^{\prime} v
$$

The quantity of water that flows past any horizontal plane is proportional to the product of the area by the mean perpendicular velocity. Thus the discharge is equal to

$$
q=\mathrm{B} \sqrt{2 g(h+k)}
$$

where $h$ is the depth of the vena contracta below the surface of the fluid in the vessel.

Newton found the ratio $\frac{B}{B^{\prime}}=\frac{1}{\sqrt{ } 2}$ nearly, whence the 
mean velocity perpendicular to the orifice is nearly

$$
=\sqrt{g(h+x)},
$$

the orifice being at the side of the vessel: thus the mean perpendicular velocity at the orifice is that due to half the depth below the surface. This ratio $\frac{\mathrm{B}}{\mathrm{B}^{\prime \prime}}$, however, depends on the nature of the orifice and the thickness of its sides; accordingly different experimentalists have given different values. Thus for the ratio* of the diameters (the square

root of the above ratio), Newton found $\frac{21}{25}$, Poleni $\frac{11}{13}$, Bernouilli $\frac{5}{7}$, Du Buat $\frac{6}{9}$, Bossut $\frac{41}{50}$, Michelotti $\frac{4}{5}$, Venturi $\frac{4}{5}$, Bidone $\frac{33}{50}$, Eytelwein $\frac{32}{50}$.

The velocity of efflux will be the same whether the hole be at the centre or the side of the vessel; for though a heavy particle of water will take a longer time in descending to the same depth by an oblique curve than by a straight line, yet in both cases it acquires in its descent the same velocity. The velocity also is independent of the form of the hole, for it merely depends on the depth below the surface. And if the orifice be immersed in water the velocity of efflux is that due to the height of the water in the vessel above that of the surrounding fluid.

2. Newton proceeds to deduce as a corollary from this theory the law of resistance of a fluid to the motion of a body in it. The case of a discontinuous fluid has already been discussed, and it has been shown that if $v$ be the 
velocity, and A the area of a great circle of a sphere moving in a fluid of density $\rho$, that the resistance will be $A v^{2} \rho$. The globe and particles are supposed perfectly elastic, and thus endued with the utmost force of reflexion. But if, on the contrary, they are perfectly hard, and without any reflecting force, the above expression for the resistance must be diminished one half. "But in continued mediums the cylinder as it passes through them does not immediately strike against all the particles of the fluid that generate the resistance made to it, but presses only the particles that lie next to it, which press the particles beyond, which press other particles, and so on, and in these mediums the resistance is diminished one other half."

If in the centre of the hole $\mathrm{EF}$ a small circle $\mathrm{P} \mathrm{Q}$, of area $\mathrm{C}$, is placed, the weight of water which it sustains

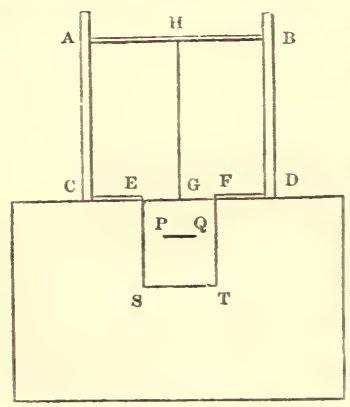
will be greater than the weight of a cone whose base is $P Q$ and altitude $H \mathrm{G}$, and less than that of a spheroid on the same base and of the same altitude. For let HP and $\mathrm{HQ}$ be the boundaries of the cataract. The cataract falls freely, and therefore there is no pressure on the sides of the mass of still

water $\mathrm{HQP}$. The pressure on the circle $\mathrm{PQ}$ is the weight of water HQP. And this will still be equal to the pressure if the ice which forms the sides of the cataract be dissolved, and the whole water be left to flow out of the orifice in any manner whatever. A little consideration will show that the mass of fluid $\mathrm{H} P \mathrm{Q}$ must have its boundaries meeting in a point at $H$, and being convex to $G$, they also meet the sides of $P$ Q at an acute angle. Therefore these boundaries lie without the surface 
of the cone and within the surface of the spheroid described on the base $P Q$, and with an altitude $H G$.

The weights of these two solids are respectively

$$
\frac{1}{3} \mathrm{C} h g \rho \text {, and } \frac{2}{3} \mathrm{C} h g \rho,
$$

where $\rho$ is the density of the fluid, and $h$ the altitude HG. Therefore the weight supported by the little circle lies between these two quantities. If the circle be very small, both these will be small, and we may take the weight supported as being very nearly equal to their arithmetic mean, that is

$$
\mathrm{P}=\frac{1}{2} \mathrm{C} h g \rho,
$$

where $\mathrm{P}$ is the pressure on the little circle. If, however, $\mathrm{C}$ be not very small, compared with $\mathrm{B}$, let us assume that the pressure is

$$
\mathrm{P}=\frac{\beta}{1-a \frac{\mathrm{C}}{\mathrm{B}}} \cdot \mathrm{C} h g \rho,
$$

where $\mathrm{B}$ is the area of the orifice $\mathrm{EF}$.

Then when $\mathrm{C}$ is very small, this must agree with the previous result, hence $\beta=\frac{1}{2}$, and when $\mathrm{C}=\mathrm{B}$ the weight supported is that of a cylinder whose base is $\mathbf{C}$ and altitude $h$, hence $\alpha=\frac{1}{2}$. Moreover, so long as $\mathrm{C}$ is less than half $\mathrm{B}$, the expression

$$
\mathrm{P}=\frac{\mathrm{B}}{\mathrm{B}-\frac{1}{2} \mathrm{C}} \cdot \frac{1}{2} \mathrm{C} h g \rho
$$

makes $\mathrm{P}$ lie between the limits assigned above.

Newton next proceeds to point out the analogy between the pressure on the circle and the resistance to a circle moving in a still fluid. Let the vessel touch the surface of 
stagnant water with its bottom $\mathrm{CD}$, and let the water run out of the cylindrical canal E F T S perpendicularly to the horizon, and let a small circle $\mathrm{P} Q$ be placed anywhere in the middle of the canal with its surface horizontal. Let $\mathrm{U}$ be the velocity of the fluid at the surface, $\mathrm{V}$ that at the orifice, $\mathrm{A}$ the area of a section of the cylinder, $\mathrm{B}$ and $\mathrm{C}$ that of the orifice and little circle $\mathrm{P} Q, h$ the altitude of the cylinder. Then

$$
\begin{aligned}
& \mathrm{V}^{2}=\mathrm{U}^{2}+2 g h, \\
& \mathrm{~V}(\mathrm{~B}-\mathrm{C})=\mathrm{UA} .
\end{aligned}
$$

If we suppose $A$ to be infinitely greater than $B$, this last equation shows that $U$ is indefinitely small, and therefore

$$
\mathrm{V}^{2}=2 g h,
$$

and the pressure on the little circle is

$$
\mathrm{P}=\alpha \cdot \frac{1}{2} \mathrm{C} h g \rho,
$$

where $\alpha$ is some quantity that becomes unity when $\frac{\mathrm{C}}{\mathrm{B}}$ diminishes without limit.

$$
\therefore \mathrm{P}=\alpha \frac{\mathrm{C} v^{2}}{4} \rho
$$

Now let the orifice of the canal E F S T be closed, and let the little circle ascend with such a velocity that the relative motion of the circle and fluid which is compelled to rush past it may be the same as when the water fell from the height HG, and the circle was at rest. The pressure on the circle will be the same as before. The velocity of the fluid will be $\frac{\mathrm{C}}{\mathrm{B}-\mathrm{C}} \mathrm{V}$, and that of the plane $\frac{B}{B-C}$ V. Let. us suppose B infinitely greater than $\mathrm{C}$, then the resistance on a plane moving with a velocity $\mathrm{V}$ in still water is $\frac{1}{4} \mathrm{C} v^{2} \rho$. If a cylinder, 
a sphere, and a spheroid, of equal breadth be placed successively in the middle of a cylindric canal, so that their axes may coincide with the axis of the canal, these bodies will equally hinder the passage of the water through the canal. The resistances will then be equal. The resistance, therefore, on a sphere will be $\frac{1}{4} \mathrm{C} v^{2} \rho$, where $\mathrm{C}$ is the area of one of its great circles.

3. The investigation of this question, as given by Newton in his first edition, was very erroneous. He had totally neglected the contraction of the vein after the fluid had passed the orifice; hence he had deduced that the velocity of the efflux was that due to only half the height of the water in the vessel. This mistake he afterwards corrected, but the investigation still remains open to very serious objections. For it is quite certain that the first case considered by Newton in which the water descends by a funnel bears no resemblance to the actual state of the motion. The water is not found to flow out in a cataract, leaving a mass of unmoved water supported by the bottom. Each particle, whether vertically over the hole or near the circumference of the cylinder, descends in a nearly vertical direction, acquiring or losing velocities in nearly the same ratio. Those particles which are once in a horizontal section remain very nearly in the same horizontal plane. When the particles approach very close to the bottom, they acquire a considerable horizontal motion, and, in consequence, the issuing stream continues to contract after it leaves the orifice. The manner in which Newton deduces the law of resistance from the velocity of efflux is also erroneous. It is very ingenious and wonderful, but at the same time very uncertain. The proposition being false in principle, we cannot expect a corollary founded on that principle to be altogether correct. The reasoning by which the resistances to a sphere and cylinder are shown to be equal can 
only be correct on the assumption that all the water above the cylinder, sphere, or spheroid, whose fluidity is not necessary to make the passage of the water the quickest possible, is congealed. This Newton himself admits. But such an assumption is by no means a legitimate one. It is also certain, by experiment, that the amount of the resistance depends very materially on the form of the surface of the body.

[See Note VII.] 


\section{CHAPTER VII.}

THE MOTION OF WAVES.

1. General consideration on the nature of Waves.

2. Waves in air - sound.

a. The nature of sound deduced from the phenomena. Scholium, Prop. L.

B. Examination of the case considered by Newton.

$\gamma$. Velocity of sound - Newton's error.

$\delta$. The manner in which sound spreads after entering through an orifice. Newton xlii.

є. The notes sounded by different pipes. Scholium, Prop. L.

3. Waves in water.

a. The motion is of the vibratory kind.

$\beta$. Newton's reasoning on this subject. Newton, xliv.

$\gamma$. Velocity of waves. Newton xlv. \& xlvi.

$\epsilon$. The nature of the motion of waves as given by a strict hydrodynamic theory. Note viii.

$\zeta$. Waves caused by the motion of a boat.

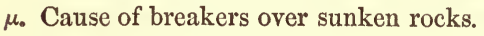

$\theta$. How the wind raises the waves.

1. To one who has never considered the nature of the motion that occasions the appearance of a wave, the idea is at first difficult. John Bernouilli the younger * declared that he could not understand Newton's proposition on this subject. The best illustration is that of a field of standing corn, because it clearly shows that, in some waves at least, there is no actual transfer of a quantity of matter. When the wind blows on the field a hollow will be seen travelling along it. This is a wave. There can manifestly be nothing

* Whewell, History of Inductive Sciences, vol.ii. p. 310, quotes Prize Dis. on Light, 1736. 
moving across the field, because everything is fastened to the ground; but there is the appearance as if something were moving along. Each particle of corn in turn descends and rises again, and, as each ear is a little later in its motion than the one in rear, the state or position in which the first was, has travelled to the second. Our first idea of a wave is, that it is a state of motion which travels along, the particles themselves only oscillating. Our second, that this state of motion may gradually change, and either increase or die away.

Suppose we have a series of particles in a straight line, let them begin to move up and down, either in this straight line, or in any parallel straight lines, so that if one be taken whose mean position, measured along the straight line is distant $x$ from the origin of measurement, its distance at the time $t$ from its mean position is represented by the formula

$$
y=a f(n t-m x),
$$

where $f$ is any functional symbol.

Then here, so long as

$$
n t-m x
$$

is constant, $y$ remains the same ; that is, a "state" travels along in such a manner that its distance $x$ at any time $t$ is given by this formula.

Differentiating we have

$$
n-m \frac{d x}{d t}=0,
$$

but $\frac{d x}{d t}$ is the velocity; hence the "state" travels with the velocity $\frac{n}{m}$; this, therefore, is the velocity of the wave. Suppose we call this $v$, then we have

$$
y=a f m(v t-x) \text {. }
$$


The "length" of a wave is the distance between two particles in a similar state of motion. If $\lambda$ be this length, $y$ must be the same for $x=x$ and $x=x+\lambda$. Now $f(z)$ will be a periodic function: suppose its values recur at intervals of $c$,

$$
\begin{gathered}
\therefore m \lambda=c, \\
\quad \text { or } m=\frac{c}{\lambda}
\end{gathered}
$$

and therefore,

$$
y=a f\left\{\frac{c}{\lambda}(v t-x) \cdot\right\}
$$

This is the type of a wave that travels in any one direction. But it can also be demonstrated that it is the general expression for all waves propagated with a uniform velocity in one direction without change of form.

There are a great variety of waves; we may have waves transmitted through elastic fluids, where a state of condensation travels along. We can have them propagated along the surface of an inelastic fluid, as water in the form of an elevation. We can have them in solid bodies, the particles of which are supposed to oscillate about their mean position, and to act on each other with forces different from those which would act if the body had been fluid. We shall briefly consider these in turn. These waves may not travel at all parts in the same direction. They may spread themselves out from a centre. In all cases a surface passing through all points in a similar state of motion is called a front of the wave. The phase of a wave at any point is the situation of the particle of that point considered as affecting its displacement and motion. Thus, two particles are in the same phase when their displacements are equal, and motions the same. They are in opposite phases when the displacement and motion of one are equal but opposite to those of the other. 
2. The true nature of sound can only be discovered by observation. We must know something about what we have to explain. The experimental facts divide themselves into three species. First, those relating to the manner in which the sounding body makes the sound. Secondly, those relating to the manner in which the sound when formed is conveyed to the ear. Thirdly, those relating to the manner in which, when the sound is thus conveyed, we hear it. In regard to the first, it is universally true that all sounding bodies are tremulous bodies. All vibrating bodies, however, do not give sound. In regard to the second, it is observed, (1.) that sound cannot be conveyed through a vacuum. Hanksbee suspended a bell in the receiver of an air-pump; the sound died away gradually as the air was removed (Phil. Trans. 1705.) - (2.) Sound is not instantaneously conveyed; the report of a gun is not heard until after the flash has been seen. But all sounds travel with equal velocity. Thus the various notes of any piece of music played at a distance reach us in perfect order. The velocity is found to vary slightly with the temperature, and at $62^{\circ}$ Fahrenheit travels at the rate of 1125 feet per second.-(3.) Sound, unlike light, does not travel necessarily in a straight line, but on entering through an orifice, as a window, spreads out in all directions, but, nevertheless, it is heard with greatest distinctness in front of the orifice. Thus the sound of a carriage can be heard round a corner, but a change in the loudness is perceptible the moment when the carriage goes behind the intervening walls.- (4.) Sound can be conveyed by solid bodies, and other fluids besides air, but in passing from one to another a considerable portion of its intensity is lost. - (5.) The intensity of sound diminishes as the distance from the sounding body increases. But when sound travels along a tube, as a speaking pipe, the gradual diminution of the sound 
is very slow.- (6.) Sound travelling through a dusty atmosphere produces no perceptible motion in it. - In regard to the third species of facts, we know but very little; it is sufficient for our present purpose that we find in the ear vibrating bodies prepared to receive the sound. These are the more obvious phenomena. They will serve as a guide to the true theory. The true test of that theory will be in its exact explanation of more refined and less obvious phenomena.

Since sound is caused by a body vibrating in air, before we invent any new theory we should prove that none of the natural consequences of such a motion will account for the phenomena of sound. Now we know that the motion of a tremulous body will disturb the air near it, and that moving air the air beyond it and so on. Thus a disturbance will be propagated on all sides in the elastic medium which surrounds the body. Also we find tremulous bodies in the ear fitted to receive such vibrations. What is more natural than to suppose that these vibrations are themselves what we call sound? To verify this it is necessary to examine strictly the nature of the disturbance in the air caused by a vibrating body. The investigation cannot be given here, but the general result is as follows. Suppose a disturbance propagated from a centre in all directions, then, - first, the intensity of the disturbance will decrease as the square of the distance increases. Thus as the disturbance was originally small, the motions at some little distance from the sounding body will be quite insensible to any of our senses except that one which was expressly adapted to receive its impressions. - Secondly, the velocity with which any wave travels is independent of the nature of the disturbance, and agrees very closely with the observed rate at which sound travels.

Thirdly, the nature of the motion of the particles is such 
that at some distance from the sounding body, the directions of oscillation of the particles will pass through the centre of disturbance supposing the propagation symmetrical in all directions, or more generally, whatever be the nature of the disturbance, the vibrations are normal to the front of the wave. In fluids no other vibrations are possible.

Of course Nerwton could not investigate completely the motion of sound. That was far beyond the power of the mathematics of his day. But, in a wonderful manner he solved to a certain degree the simpler case of the motion of the air in a tube. The principle he used, and the present mode of reasoning on this subject, are both illustrated in the following analytical view of the investigation.

Whatever the motion of the air may be, we suppose the tube so small that we need not consider any motion except that which is along the length of the tube, and this motion will be the same for all particles in the same perpendicular section of the tube. Suppose, then, that at the time $t$, the particle which when the air was at rest was at a distance $x$ from the origin of measurement is at the distance $x+\xi$. Then an element of air whose length had been $d x$, is now $d x+d \xi$, and as the mass must remain the same, the density which was $D$ is now

$$
\rho=\frac{\mathrm{D}}{1+\frac{d \xi}{d x}}=\dot{\mathrm{D}}\left(1-\frac{d \xi}{d x}\right) \text { nearly. }
$$

The square of $\xi$ may be neglected, for we know that that particular motion of the air which we call sound, whatever it may be, is very small. Also the pressures on the two sides of the element are, when reduced to a unit of area,

$$
p \text { and } p+\frac{d p}{d x} d x
$$


and since the pressure varies as the density we have

$$
p=x p,
$$

and therefore the moving pressure on the element is

$$
-x \frac{d \rho}{d x} d x
$$

But the mass moved is $\mathrm{D} d x$; hence on substitution for $\rho$, the accelerating force on the element is

$$
x \frac{d^{2} \xi}{d x^{2}}
$$

Thus the force depends on the displacement, and the displacement, in its turn, on the force. If we assume a form for the displacement, and then show that this displacement leads to a force that will produce this exact displacement, we have discovered a possible motion. And if this displacement also agree with all the other conditions of the question, we have discovered the actual motion. For it is clear that from a given disturbance under given circumstances, only one kind of motion can result. Newton assumes accordingly that

$$
\xi=a \cdot \sin (n t-m x) .
$$

The accelerating force is then, by two differentiations,

$$
\begin{aligned}
& -x m^{2} \cdot a \sin (n t-m x) \\
& =-x m^{2} \cdot \xi
\end{aligned}
$$

that is, the force varies as the distance from a fixed point' and urges the particle towards that point. This force is well known to lead to the very form for $\xi$ that we started with, (Prop. xxxviii. of Book I.) provided

$$
n^{2}=\times m^{2} \text {, }
$$

or the velocity $\left(\frac{n}{m}\right)$ with which the wave is propagated is $\sqrt{x_{0}}$ This, therefore, is the velocity of sound. 
As an example of a case in which the motion is actually represented by this law, let us take an infinite tube, and suppose the air in a small part of it to be set in motion, so that it will begin to move according to the form we have assumed for $\xi$. Let the extremities of this part of the tube be $\mathrm{A}$ and $\mathrm{B}$, the particles $\mathrm{A}$ and $\mathrm{B}$ and all the remainder of the air in the tube is supposed to be at rest. Let us now consider the motion during a small time $\delta t$. The particles between $\mathrm{A}$ and $\mathrm{B}$ will, by the above reasoning, continue to move according to the law assumed for $\xi$. The velocity and condensation, therefore, being represented by $\frac{d \xi}{d t}$ and $-\frac{d \xi}{d x}$ will be respectively

$$
-a n \sin (n t-m x) \text { and } a m \sin (n t-m x) \text {. }
$$

Let $x$ be measured in the direction from $\mathrm{A}$ to $\mathrm{B}$, which we shall suppose from left to right. The particle $A$ is at rest, the condensation at that point and for all points on the left of $\mathrm{A}$ is zero. Take a particle $a$ on the right of $\mathrm{A}$, where A $a=\delta x$, the condensation at that point is indefinitely small and is decreasing. The particle A will, therefore, remain at rest. The particle $a$ will come to rest, that is, be in the same situation that $A$ is in, at the end of the time $\delta t$, where

$$
n \delta t=m \delta x,
$$

that is, the left end of the pulse travels onward with a velocity $\frac{n}{m}$, leaving the air behind it undisturbed. By similar reasoning, it can be shown that the right end of the pulse travels onward with the same velocity. At the end of the time $\delta t$, the pulse will merely have advanced a space $\delta x$, and the circumstances of the motion will be the same as before. The same motion will, therefore, be 
repeated in the next interval, and so on throughout all time.

Thus we see a single pulse can be propagated along the tube without any change in magnitude, form, or time of vibration. In order to form these pulses let us imagine a plate to be placed at one end of the tube, vibrating according to the law

$$
\xi=a \sin n t,
$$

then each pulse of the plate as formed will be propagated unaltered along the tube, and the motion at any distance $x$ from the plate will be given by the law

$$
\xi=a \sin (n t-m x) \text {. }
$$

The velocity of sound is independent of the values of $m$ and $n$. These are the only constants on which the difference between two notes could depend. Whatever, then, is the pitch of a sound, it will travel with the same velocity.

The numerical value of the velocity of sound depends on the constant $x$. This expresses the constant ratio of the elastic force or pressure of the air to its density. The density of the air Newton calculated to be about $\frac{1}{11890}$ th part of the density of quicksilver, and the pressure of the air is equal to the weight of a column of mercury about 30 inches high. Hence the ratio of these two is equal to

$$
g \times \frac{30}{12} \times 11890,
$$

the units being feet and seconds of time. The square root of this, which is the velocity of sound, is 979 . Sound, therefore, should travel at the rate of 979 feet per second. The elastic force $p$ for a given density being increased by an increase of temperature, the result thus obtained should be too small in hot air and too great in cold. But this 
result should be true about the temperature of spring and autumn.

When Newton completed this investigation the true velocity of sound was not accurately known. From some rough experiments, conducted by himself, he believed that this result was really near the truth. But subsequent experiments showed that it was erroneous by 163 feet. This was a very serious error, and Newton tried to explain it away, by saying that no allowance had been made either for the crassitude of the solid particles of the air, or the presence of vapours. He even attempted to show that on taking these into account, the calculated and observed velocities were in close agreement. Such explanations are, however, unsatisfactory, and unless some other explanation had been found, the theory would stand in direct opposition to experiment. The theory of sound continued to advance by the labours of the great mathematicians who followed Newton; the motion of sound in a tube was investigated without any assumption as to the nature of the motion, but the discrepancy still remained unexplained.

This was reserved for Laplace, who remarked that in rapid vibrations, the sudden rarefactions and condensations of the air must affect its temperature, and therefore its elasticity. The amount of this must be determined by experiment, and it was shown that if $s$ represent the condensation, the form to be used must be, not as heretofore

$$
p=x \mathrm{D}(1+s)
$$

but

$$
p=x(1+\beta) \mathrm{D} \cdot(1+s),
$$

where $\beta$ lies between $\cdot 3748$ and $\cdot 4$. The velocity of sound, therefore, is not $\sqrt{x}$, but $\sqrt{x(1+\beta)}$. The agreement T 2 
of the observed and calculated velocity was now found to be very close.

The value of $x$ depends on the general temperature of the air. By Amontem's law, we know that if $x$ be the value at any temperature, the value at any other exceeding this by $\theta^{\circ}$ will be

$$
x=x_{0}(1+\alpha \theta) \text {, }
$$

where $\alpha=\frac{1}{490}$. If, therefore, the temperature of the air be $\theta^{\circ}$ above $60^{\circ}$, the velocity of sound will be 1124 $\left(1+\frac{\theta}{900}\right)$ feet nearly.

The demonstration we have given of Newton's proposition of the motion of sound in a tube may easily be extended into a vigorous demonstration. For the accelerating force on any element having been shown to be

$$
\varkappa(1+\beta) \frac{d^{2} \xi}{d x^{2}}
$$

the equation of motion must be

$$
\frac{d^{2} \xi}{d t^{2}}=\alpha^{2} \frac{d^{2} \xi}{d x^{2}}
$$

putting $\alpha^{2}$ for $x(1+\beta)$. This equation must be true for the motion of sound in a tube under all circumstances. Thus, the tube may be finite, open or closed, and the disturbance may be caused in any manner. The complete integral of this equation is known to be

$$
\xi=f(\alpha t-x)+\psi(\alpha t+x),
$$

where $f$ and $\psi$ are unknown functions depending on the nature of the disturbance and the other circumstances of the tubc.

This investigation of the motion of sound in the air depends on the assumption that the medium is a perfect fluid. But this is not the case. The effect of internal 
friction is found to consist partly in a diminution of the velocity of propagation, and partly in a more rapid diminution of the intensity than would correspond to the increase of distance from the centre of divergence. The diminution of velocity is found on calculation to be so small, that it is less than one foot in 1,578,000 miles. The change of intensity, though not so utterly insignificant as the change of velocity, is found to be still insensible. Such small differences as these may be altogether neglected.

It is well known that sound does not always travel in a rectilinear direction. "Sounds," Newton remarks, "may be heard though a mountain be interposed; and if they come into a chamber through the window, dilate themselves into all parts of the room, and are heard in every corner, not as reflected from the opposite walls, but. as directly propagated from the window, as for as our senses can judge." Let us imagine a series of waves to be advancing directly through an orifice, and let the aperture be divided into small elements. Each element may be considered as the origin of a series of disturbances. The disturbance in any part of the room will be made up of those propagated from all the several elements. Now, the motions being all small, it is a well known principle that the actual disturbance will be the sum of all those that would have been propagated from each separate element on the supposition that there was no other motion at the same time in the medium. Each separate disturbance may be calculated by the known rules according to which any motion spreads itself on all sides. The actual disturbance, on which the sound depends, will be represented by a definite integral. It is needless to go through the work, but the result is, that, provided the length of a wave be not very small compared with the orifice - and this is the case in sound, and the waves on the surface of water-the. 
motion will be sensible at other points of the room, as well as directly in front of the orifice. Thus Newton's expectation was confirmed, though he was ignorant of the condition on which it depended. But if the length of the waves are indefinitely small compared with the size of the orifice,-and this is the case in the Undulatory Theory of Light, - then it is found that, excepting directly in front of the orifice, the definite integral is altogether insensible, the condensations and forward motions of one wave being superimposed on the rarefactions and backward motions of another in such a manner that there is no sensible disturbance. Such waves must be considered as being propagated in rectilinear directions.

The first step in the theoretical explanation of the sounds produced in pipes was made by Newton. He remarks that Sauveur found by experiment that an open pipe about five Paris feet in length gives a sound of the same tone with a viol string that vibrates a hundred times in a second. Therefore, he argues, there are near one hundred pulses in a space of one thousand and seventy Paris feet, which a sound runs over in a second of time; therefore one pulse fills up a space of about $10_{\frac{7}{1} 0}$ Paris feet, that is twice the length of the pipe. From whence it is probable that the lengths of the pulses in all sounds made in open pipes are equal to twice the length of the pipes. Newton did not examine any further into the subject, but leaves it for others to carry out the theory. Lagrange and Bernoulli were the first to give more minute explanations of the leading facts. Since that time, Euler, Lambert, Poisson, have developed the subject still further.

The theory is to a great extent included in the equations to the motion of sound in a tube which we have already given. It can be shown from these that the motion is made up of two waves continually travelling along the tube 
in opposite directions. Of these one may often be considered as the reflection of the other at the open or closed end of the tube. These two waves will "interfere" as it is called, and there will be in consequence two sets of points in the tube, which possess remarkable properties. At any point of one set there is no motion in the air, but only a condensation. At any point of the other set there is no condensation, but only motion. The first are called "nodes," the other "loops." These points are placed in regular order, alternately a node and loop at distances one fourth the length of a wave.

Suppose a tube closed at one end to be sounding a note in unison with a vibrating plate at the other. Then clearly, since the air must remain in contact with the tube at the closed end, there can be no motion there. That point must be a node. Since the air moves in consonance with the vibrating plate, there must be no "action or reaction" between the air and plate. If there were, the sounds would not be in unison, and the note of both the tube and plate would begin to change. The pressure of the air must therefore be the same on each side of the plate; therefore there is no condensation in front of the plate. That point must be a loop. One end of the tube is therefore a loop, the other a node. Hence the length $(l)$ of the tube must be an odd multiple of $\frac{\lambda}{4}$, where $\lambda$ is the length of a wave. That is,

$$
l=(2 n+1) \frac{\lambda}{4}
$$

That note which has the largest value of $\lambda$ which can be sounded from a given pipe is called the "fundamental" note of that pipe. For this note $n=0$, and we have

$$
\lambda=4 l \text {. }
$$

The pitch of a note being determined by the length of the 
wave which forms it, all the notes that can be sounded from the same closed tube will form the series

$$
\begin{array}{llllll}
1 & \frac{1}{3} & \frac{1}{5} & \frac{1}{7} & \frac{1}{9}
\end{array} \text {, \&c. }
$$

for we have merely to give $n$ all its values from nothing to infinity, to get all the possible values of $\lambda$.

Suppose now the tube to be open at the end opposite to that by which the air is set in motion. It is assumed that at the open end the density of the air must be the same as that of the surrounding air, a fact that is not exactly true. Taking it for granted, it will follow that both ends of tube must be loops. The length of the tube must therefore be an even multiple of $\frac{\lambda}{4}$, that is

$$
l=n \frac{\lambda}{2} \text {. }
$$

The fundamental note corresponds to $n=1$ or

$$
\lambda=2 l,
$$

and all the notes that can be sounded from the same open tube will form the series

$$
1 \frac{1}{2} \frac{1}{3} \quad \frac{1}{4} \quad \frac{1}{5}, \& \mathrm{c} \text {. }
$$

We also learn that the note of the pipe being measured by the value of $\lambda$, it will be proportional to the length of the pipe, and that to get the same note from two pipes, one closed and the other open at the end opposite to that at which the air is set in motion, the second must be double the length of the first.

The theory of stringed instruments is in many instances remarkably similar to that of wind ones. The equations of the oscillations of a stretched string are exactly the same as those of a sounding pipe.

Much progress has been made by the experimental researches of Hopkins, Chaldni, Savart, Willis, and others. 
But we have no space to do more than merely allude to their labours.

There are a great many other waves propagated through the air besides those which can be heard. Our ear is a musical instrument that vibrates in consonance with all notes whose periods lie between two limits. All other sound waves therefore, though their theory is exactly the same, are not heard.

Besides these sound waves, there are other great waves which traverse the air in all directions, and whose passage is indicated by the variations of the barometer. These waves are very large, and pass over whole continents in their course, and seem to recur with some regularity. Their theory is different from that of sound, and as yet imperfectly understood. The examination of the "facts" connected with them was begun by Herschel, and has been since continued with much success by William Birt.

So also the waves that produce sound can travel through other substances besides air. The theory is in some points different from that we have been considering.

3. That the elevations on the surface of water which we call waves are not really the transmission of a body of water, but merely the movement of a particular state of motion, will be obvious on very little consideration. First, by experiment, a piece of cork floating on water agitated in this manner is found merely to oscillate, and does not advance as the wave moves onwards. Secondly, by theory, such a disturbance being supposed given to the water, as we know by experience will produce the appearance of waves, the principles of Hydrodynamics lead us to the conclusion that the motion is really of a vibratory kind. 
On this subject Newton has not many propositions. He has shown an analogy between the motion of waves and the oscillation of water in a pipe. Let there be a column of fluid of length $l$ in the $\mathrm{U}$ pipe $\mathrm{MA} m$ : let the water when at rest stand at the level $\mathbf{M}$. Then if it be raised above this level in one leg to the height $\mathrm{P}$, it will be depressed an equal amount in the other, say to $\mathrm{Q}$. The weight of water tending to pull it back again will be equal to twice the weight of the column P ; that is, the force varies as the displacement, and tends to pull the body back to its original position. This case of motion has been investigated in the First Book, and the result is, that the motion is vibratory, the time of oscillation will be always the same, and equal to $2 \pi$ divided by the square root of the ratio of the accelerating force at any distance to that distance. But the moving force is twice the column $\mathrm{P} \mathrm{M}$, and the mass moved is the column $l$. Hence the time will be

$$
\pi \sqrt{\frac{2 l}{g}}
$$

or it varies as the square root of the length of the column of water.

Now, in waves the motion is carried on by the successive ascents and descents of the water; hence the time that must elapse before any particle now at the top of a wave will again be the top is

$$
\pi \sqrt{\frac{2 l}{g}}
$$

where $l$ must here be assimilated to half the length of a wave. We cannot say they are equal to it, but they will increase or decrease together. Newton assumes 


$$
l=\frac{\lambda}{2},
$$

and therefore the time of oscillation is

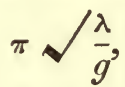

where $\lambda$ is the length of a wave, measuring either from top to top or hollow to hollow. This expression for the time is the same as that for the oscillation of a simple pendulum whose length is $\lambda$. Hence a wave whose length is equal to the length of a simple pendulum should advance a space equal to that length in the time of one vibration of that pendulum. The velocity of the wave will therefore be

$$
\frac{1}{\pi} \sqrt{\lambda g}
$$

Thus the velocity is independent of the magnitude of the waves, or the density of the fluid. It is greater for long waves than short ones. The waves of sound in air travel with equal velocity, whatever be their length. The undulatory theory of light requires us to believe that, except when light goes through a vacuum, the waves of different length travel with different velocities.

Newton does not propose this as more than a first attempt. He says, "These things are true on the supposition that the particles of water ascend and descend in a right line. But, in truth, that ascent and descent is performed in a circle, and therefore I propose the time defined by this proposition as only near the truth." In modern times the motions of waves have been more accurately investigated. If $h$ be the uniform depth, $\lambda$ the length of the waves, $v$ the velocity of propagation,

$$
v=\sqrt{\frac{g \lambda}{2 \pi}} \cdot \sqrt{\frac{1-\varepsilon^{-\frac{4 \pi}{\lambda}}}{1+\varepsilon^{\frac{-4 \pi}{\lambda}}}} .
$$


If the depth be very great we have

$$
v=\sqrt{\frac{g \lambda}{2 \pi}} \text { very nearly, }
$$

and when the wave is very long,

$$
v=\sqrt{g h} \text { very nearly. }
$$

The former of these results is not the same as Newton's, but the general conclusion will be unaltered. The exact result may, however, be obtained by Newton's reasoning, if we slightly change the value assumed for $l$ and take

$$
l=\frac{\lambda}{\pi}
$$

The second result admits of an easy demonstration, which we shall speak of in another place.

[See Note VIII.] 


\section{CHAPTER VIII.}

THE THEORY OF THE TIDES.

I. Newton's investigation on the Tides.

a. The tides considered as a question in the motion of fluids, deduced from the Lunar Theory.

B. General explanations of eight phenomena of the tides.

$\gamma$. The calculation of the height of the lunar and solar tides.

$\delta$. The tides in the moon.

II. The theories that have been proposed since Newton's time. Note IX.

1. The Equilibrium Theory.

a. Its fundamental hypothesis.

$\beta$. The results of calculation made according to this theory, - three kinds of tides.

$\gamma$. Airy's opinion of this theory.

2. The Hydrodynamic Theory.

$\alpha$. Its fundamental assumptions.

B. The results of calculation, - three kinds of tides.

3. The Wave Theory.

a. Consideration of ocean tides.

$\beta$. River tides, results of calculation and explanation of the chief phenomena.

$\gamma$. Where this theory fails.

III. Some results of observation. Note IX.

The cause of the tides in the ocean have always excited the curiosity and wonder of mankind. Their regularity, the magnitude of the scale on which they take place, the difficulty of conceiving what that power could be that can raise twice a day so vast a body of water, all render the question one of the most interesting in the whole range of science. Phitheas, it is said, was the first who remarked that the tides followed the course of the moon. But the "why" was still as great a mystery as before. Galileo 
thought he could explain it by a combination of the rotation of the earth about its axis with the annual motion round the sun. The impossibility of deducing an explanation from these premises of the most ordinary phenomena of the tides is evident from what we have said in a previous chapter. Galileo, busy in establishing by new proofs the rotation of the earth, was naturally inclined to find in it the cause of a phenomenon so mysterious, which if it had succeeded would have furnished him with a most powerful argument. Descartes had another equally impossible theory; it was reserved for Newton to suggest the true cause of the motion of the sea. The discoverer of gravitation could not be long before he saw that whether or not it was the only cause of the tides, it must certainly be one of them. The attraction of the moon could not be the same in all parts of any extended sea. Motion, therefore, must ensue. Nor could any position of rest be ever assumed, because the earth and moon themselves are in motion. And here Newton showed his superiority over those philosophers who afterwards treated of the same subject. He saw that the motion of the tides was a question of Hydrodynamics, and in his First Book he considers it as such, and has even shown that in one particular case the water would be lowest in that part which is immediately under the moon. That he did not do more is no reproach. Even at the present day the theory of Hydrodynamics is in its infancy: how impossible then it must have been in Newton's time, when the simple laws of the motion of a single particle had only just been understood, to have attempted the consideration of a fluid under the action of complicated forces. Newton gives, therefore, merely a general explanation of the tides, and enters into some numerical calculations merely as a first attempt. 
Let us imagine that a uniform channel is cut round the earth at the equator and filled with water: let it be supposed that this fluid is disturbed by the action of the sun or moon; supposed for simplicity also to move in the equator. Newton endeavoured to discover in what manner the water would move in this imaginary channel, Book I., Prop. Lxvi., Cor. 19. In considering the motion of a satellite supposed when undisturbed to describe a circle round the earth, Newton has shown that the disturbing force will in two different ways cause the orbit to become slightly oval, the earth remaining in the centre and the greater axis being perpendicular to the straight line joining the sun and the centre of the earth. This is somewhat parallel to the case in point. If each particle of water were kept in its place by its centrifugal force they would tend to rise at the quadratures and sink at the syzygies, they would be swifter at the syzygies and slower at the quadratures; they would ebb and flow in its channel after the manner of the sea. But the analogy is not perfect, for the water is supported, not by its centrifugal force, but by the channel in which it flows. We see, however, that an ebbing and flowing of the sea will be produced, though the points of greatest and least height of water may be different. This reasoning applies whether the disturbing body be the sun or moon.

Though, therefore, by this reasoning we cannot deduce a perfectly accurate theory of the tides, yet we are able to perceive that the sea ought to rise and fall twice in each day. The moon will form at any place two high tides and two low tides in the interval between leaving the meridian of that place and returning to it again, that is, in a lunar day. The sun will also at the same place form two high tides and two low tides in the interval between leaving the meridian and returning to it again, that is, in a solar day. 
These two will not appear separately, but a mixed tide will result from the two. Since both are small, we may consider the actual tide as being the sum of the two tides that would have been formed had the disturbing bodies acted alone. The force of the moon to raise the tides is much greater than that of the sun, as we shall presently see, so that for most purposes we may altogether neglect the solar tide, or rather regard it as a small correction to be applied to results calculated on the supposition that the moon alone acted to raise the tide.

First. The lunar tide follows the moon at a given interval, called the "establishment." Hence as the moon by her proper motion rises every day about forty minutes later than on the preceding day, the high tide will be as much later every successive day. But more minute investigation shows that the high tide does not follow the moon's passage over the meridian by any constant interval. We must make a small correction for the effect of the solar tide. If the sun follow the moon across the meridian, the solar high tide will follow the lunar high tide, and the actual high water formed by the union of the two will lie between these two tides, and will therefore follow the moon's passage across the meridian at an interval slightly greater than the "establishment." So if the sun precede the moon the interval will be less. Therefore, when the moon is in the first and third quarters, the high water is a little later, when in the second and fourth a little earlier, than the establishment would indicate.

Secondly. At or about new and full moon, the high tide formed by the sun coincides with that formed by the moon, hence the high tides will be higher and the low tides lower than when the moon is in any other position relative to the sun. These are called Spring Tides. At or about the half moon, the high tide formed by the sun coincides with the 
low tide formed by the moon. They tend to neutralise each other. The high tides will be lower and the low tides higher than at other times. These are called Neap Tides.

Thirdly. The effects of the luminaries depend on their distance and vary inversely as the cubes of their distances. Hence the respective tides of the sun or moon are greatest when the luminary is in perigee and least when in apogee. In winter the solar tides are therefore greater than in the summer, and the lunar tides have like changes every fortnight.

Fourthly. The effects of the disturbing bodies depend on their declinations. If the moon were at the pole, it would attract the water without any daily remissions of its action. The water would assume a position of equilibrium, and there would then be no daily tides. Thus as the luminaries decline from the equator, their effects become less and less.

Fifthly. The river tides are formed by the propagation of the tidal disturbance from the seas where they were formed. In these therefore the greatest tides occur later than they should do according to the above statement.

Sixthly. The effects of the disturbing bodies depend on the latitude of the place. Suppose the attraction of the moon to raise a tide, one of whose highest points is in latitude $a$, therefore the other is in the other hemisphere at the same latitude. This state of tide in one day will travel round the earth, the vertices always remaining at the same latitude $a$. Then it is clear that the altitude of the tide at any place will depend on its distance from a vertex of this heap of waters. That place will have the highest tide over which the vertex passes, and the height at any other place will be less and less the greater the angular distance from the vertex; that is, the greater the difference of latitude between the place and one vertex. The tide will therefore be least at the equator and poles, and 
greatest in latitude $a$; thus the height of the tide varies with the latitude.

Seventhly. But the difference between the latitude of the place and one vertex is not the same as that between it and the other vertex. Hence, though we have two tides every day, these two tides will be of unequal magnitude. The moon's orbit is inclined at but a small angle to the ecliptic; hence, speaking generally, her orbit will have nearly the same position relative to the equator that the sun's orbit has. A line drawn through the centre of the earth and moon is the axis of the tidal spheroid. The tides which occur on the side next the sun, when the sun has north declination, will be greater than the tide on the opposite side of the earth. Therefore in summer the day tides are greater than the night tides; similarly in winter the night tides are greater. "If the pole of the tidal flood follow the moon, say at six hours, the pole will be north from the time the moon is six hours west of the sun to the time when she is six hours east, that is, from the time when the high tide is at noon to the time when it is at midnight." In such a case therefore the afternoon tide is greater than the morning tide in summer and less in winter. Similar reasoning will apply when the "age of the tide" is twelve, thirty, \&c. hours.

Eighthly. If the tide be brought to any place by two unequal channels, the two tides following the two transits of the luminary across the neighbouring oceans will meet one another at this place, and form a compound tide. Suppose one tide to be delayed six hours, it will be in exact opposition to the tide which arrives by the other path. If the two were equal, the waters would stagnate, and there would be no tide at this particular point. But if the luminary be not in the equator, the two tides are unequal; hence, compounding the two, there will be one tide every twenty-four hours.

Newton quotes from Halley, as an example of this, the 
port of Batsham, in Tunquin, lat $20^{\circ} 50^{\prime} \mathrm{N}$. The tide arrives by two inlets, one from the seas of China, between the continent and the island of Leuconia; the other from the Indian Sea, between the continent and the island of Borneo. "The tide begins every successive day later by about three quarters of an hour; so that in fifteen days the time of high water advances from one o'clock in the afternoon, for instance, to twelve at night; after which it does not advance to one in the morning, but falls back thirteen hours to twelve at noon, and so on perpetually. In this way the high water is always in the afternoon during the summer half year (March to October), and in the forenoon during the remaining half. About the time when the tide falls back thirteen hours, the tides are very small and scarcely perceptible; at the intermediate times they are greatest." (Phil. Trans. 1833, page 224. Whewell.)

Let us now follow Newton in his attempt to calculate numerically the forces of the sun and moon to raise the tides. He first refers to his Lunar Theory for a calculation of the force of the sun to draw the moon towards the earth. When in quadratures this force is $\frac{1}{63809406} g$, where $g$ is the force of gravity at the surface of the earth. When in syzygy the force is double this quantity. But the disturbed bodies are here the particles of water at the surface of the earth, which are nearer the earth than the moon in the ratio of $60 \frac{1}{2}$ to 1 . These forces must, therefore, be decreased in the same ratio. The force in quadratures is therefore $\frac{1}{35606600} g$, and that in syzygy double this quantity. The first depresses the water in quadrature, the second raises that in syzygy. Hence the two produce the same effect, and the whole force to raise the sea will be the sum of the two, that is, thrice the torce in quadrature. 
The force of the sun, therefore, to raise the tide, will be $\frac{1}{12865200} g$. But the centrifugal force has been shown to be $\frac{1}{289} g$. The latter raises the water to a height under the equator exceeding that under the poles by 85,472 Paris feet. Hence, the sun's force is $\frac{1}{44527}$ th part of the centrifugal force, and will raise the water $\frac{85472}{44527}$, or 1 Paris foot and $11 \frac{1}{8}$ inches, about two English feet.

If the mass of the moon had been known in Newton's time, he might have made a similar calculation to determine its force to raise the tides. But he was obliged to deduce this mass from the height of the tide itself. "Before the mouth of the river Avon, three miles below Bristol, the height of the ascent of the water in the vernal and autumnal syzygies of the luminaries (by the observations of Sturmy), amounts to about 45 feet; but in the quadratures to 25 only. The former of these forces arises from the sum of the forces of the sun and moon, the latter from their difference."

If, therefore, $\mathrm{L}$ and $\mathrm{S}$ are supposed to represent respectively the forces of the sun and moon while they are in the equator as well as in their mean distances from the earth, we shall have $\mathrm{L}+\mathrm{S}$ to $\mathrm{L}-\mathrm{S}$ as 45 to 25 , or $\mathrm{L}$ to $\mathrm{S}$ as 7 to 2. Newton remarks that the observations at Plymouth by Colepress gave a ratio 41 to 23, a proportion which agrees tolerably well with the former. $\mathrm{He}$, however, prefers the former result, because the observations were made on larger tides. This reasoning proceeds on the supposition that the earth is without rotation, and in that case there would be high water immediately under the luminary. But this is not the case ; it does not occur until three hours after the transit of the luminary. Neither does the highest tide occur at the syzygy, but about three days after. Newton attributes this to the "force of reciprocation," which the water once moved retain a little 
while by their vis insita. The luminaries continue to act with great power for a little while after the moment of their greatest strength, and thus continue to raise the tides. But Laplace remarks (Méc. Cel. xiii., chap. 1.) that "vraisemblable" as this is, it is nevertheless erroneous, for an accurate investigation shows that, notwithstanding this continued action of the luminaries, the greatest full tides should occur exactly at the syzygies and the least exactly at the quadratures, and that therefore the explanation of the delay must be sought for in the accessory circumstances. It is, in fact, due to friction.

Newton then proceeds to make several "corrections" to this result which he conceives to be necessary. He observes that the luminaries are not in the positions of greatest efficiency at the moments of the greatest high tide at the place under consideration; that therefore it is not the whole force of these luminaries that is employed to raise the tide, but this force multiplied by the cosines of certain angles. He corrects, therefore, for the instantaneous angular positions of the luminaries at the moment of the greatest tide. He also remarks that changes in the distance of the moon are produced by the inequality in her motion called the "Variation," and he adds, therefore, another correction for the distance of the luminary at the moment of the greatest tide. But Laplace points out that this correction also is wrong. In fact it contradicts the previous reasoning, for if the tide be due to the accumulated action of the luminaries during a certain instant, we must not consider it as proportional to the force at the end of that interval. But when all corrections are applied, he finds that the sun's force raises the tides by 1 foot $11 \frac{1}{\overline{3} 0}$ inches, and the moon's force will therefore raise the same to the height 8 feet $7 \frac{5}{2} \frac{2}{2}$ inches, and the joint action of the two to the height $10 \frac{1}{2}$ feet, and when the moon is 
in its perigee to the height of $12 \frac{1}{2}$ feet, and more, especially if the wind sets the same way as the tide.

Newton makes some remarks on the observed nature of the tides in different parts of the world. His explanations are not always perfectly correct. To have a full tide raised, an extent of sea from east to west is required of no less than 90 degrees. Hence, he infers, the tides in the Pacific are greater than those in the Atlantic, and those in the North Atlantic than thọse within the tropics. In some ports, where the water must be forced in and out through narrow channels, the flood and ebb must be greater than ordinary, and this force of efflux, being once given to the water, may, he argues, continue until it raises the tide as much as fifty feet. But on such shores as lie towards the sea with a steep ascent, where the waters may freely rise and fall without that precipitation of influx or efflux, the proportion of the tides agrees with the forces of the sun and moon.

The force of the moon being only $\frac{1}{2871400}$ th part of gravity at the surface of the earth, will not be sensible in any statical or hydrostatical experiment, or even in those of pendulums. It is in the tides only that this force shows itself.

I have already mentioned that the mass of the moon was unknown in Newton's time. He makes use of the observations on the tides to determine her mass and density. Her force is $\frac{1}{4 \cdot 4815}$ th part of the sun's force to raise the tides. And, by lunar theory, these forces vary as the masses of the attracting bodies directly and the cubes of their distances directly; that is, as their densities and the cubes of their apparent diameters. These diameters being $31^{\prime}, 16 \frac{1}{2},{ }^{\prime \prime}$ and $32^{\prime}, 12^{\prime \prime}$, the ratio of the densities is 4.891 to 1 . But the density of the sun was known to be one-fourth that of the earth. Hence the moon is denser 
than the earth in the ratio of 11 to 9 . The true diameter of the moon is to the true diameter of the earth (according to Newton) as 1 to $3 \cdot 65$. Hence the mass of the moon is $\frac{1}{39783}$ (or nearly $\frac{1}{40}$ th) that of the earth. It is now known that the true mass is about $\frac{1}{49}$ th that of the earth.

If the moon's body were fluid like our sea, the force of the earth would raise tides in it. The tide in the moon caused by our earth is to the tide in our sea caused by the earth as the mass of the earth to the mass of the moon. This ratio Newton imagined to be 1 to $39 \cdot 788$. Hence he concluded the lunar tide was 93 feet. Upon this account the figure of the moon would be a spheroid whose greatest diameter produced would pass through the centre of the earth and exceed the diameters perpendicular by 186 feet. It might be supposed that the tides in the moon must be overwhelming. But as the moon always turns the same face to us, the tide is stationary, and would therefore merely affect the permanent surface of the lunar ocean. But no sea has ever been detected in the moon. If the moon had been originally fluid, or had been created in a form which would have no tendency to break up, her longest diameter must point to the earth. Hence the same face of the moon would always appear to us, and the body could not rest in any other position, but would always return by a slow vibratory motion.

[See Note IX.] 


\section{CHAPTER IX.}

THE CIRCULAR MOTION OF FLUIDS.

1. The hypothesis of the Cartesian theory.

2. Newton's hypothesis as to the law of internal friction in fluids, - the motion of a cylindrical vortex - Bernoulli's objections to this result. Prop. LI.

3. Some difficulties of the Cartesian theory which are considered by Newton.

a. The Sun's rotation. LII. Cor. 4.

$\beta$. The third law of Kepler. Scholium LIII.

$\gamma$. The two first laws of Kepler. Scholium LIII.

$\delta$. The density of the planets, Lirr.

$\epsilon$. The disturbance of the Sun's motion by the planetary vortices. LII. Cor. 5, \&c.

1. Before the time of Newton the Cartesian theory was believed in by almost every nation of Europe. It was therefore necessary that some notice should be taken of these opinions in a work in which a totally different system was proposed for the first time. Accordingly Newton has devoted a section to the consideration of this hypothesis. 'The philosophy of Descartes was naturally a very popular one. Its explanations of the general facts of astronomy were so exceedingly simple that it required no previous learning to enable any one to understand them. Men were pleased to think that in a few minutes they could learn the cause of the motion of the planets. The sun was supposed to be the centre of a vast vortex or whirlpool, the density and angular velocity of the various parts of which were different. The planets being placed, each in that 
stratum whose density was equal to its own, were thus dragged round the sun. Those planets which had satellites were themselves the centres of smaller whirlpools in which their secondaries revolved according to the same laws by which they themselves were carried round the sun. The ellipticity of the orbits were accounted for by supposing the vortices themselves not circular. Such was the theory as given by Descartes. It was open to so many objections that it was greatly changed in character by his successors. Newton proved that the original theory could not be made to agree with Kepler's laws. Bernoulli * imagined the vortices to be circular, and accounted for the elliptic path of the planet by a combination of an oscillatory movement with the circular motion of the whirlpool. Bouguer then showed that the two portions of the curve which the planet would describe in its oscillations from aphelion to perihelion would not be equal or similar. D'Alembert showed that an elliptic vortex was, under the circumstances of the case, impossible. The theory was always unsatisfactory: all sorts of suppositions were made in vain by Huygens, Perrault, Villemot, Mollieres, Gamaches, \&c. They never could explain one phenomenon without contradicting another.

Newton of course considers the Cartesian theory as it was originally given by its author. We shall confine ourselves within the same limits. The theory has no longer any adherent, and there can be no advantage in attacking that which no one defends.

2. If the particles of a fluid did not exert any action on each other, a vortex in which the velocity was any function of the distance would be possible. For suppose the whole fluid in any cylinder to be revolving round the axis; 
describe two indefinitely near cylinders with this straight line as their common axis. The fluid between these two cylinders will evidently revolve unchanged whatever be its velocity, and however different from that of the neighbouring fluid, the normal pressure being sufficient to counteract the centrifugal force. There is, however, no perfect fluid in nature: if a stream of one fluid be made to pass through another, it will carry the particles of the second along with it. A true theory of vortices must take account of this "internal friction" of the fluid. Newton starts with the hypothesis that "The resistance arising from the want of lubricity in the parts of a fluid is, cæteris paribus, proportional to the velocity with which the parts of the fluid are separated from each other."

This hypothesis, as Newton himself remarks, is probably not altogether correct, but, nevertheless, there can be no doubt that it will give us a general idea of the motion. The first problem to which we shall apply it will be the following:

Two infinite cylinders having a common axis revolve in any uniform manner about that axis. Fluid is placed between them, and soon acquires a rotatory motion; it is required to determine what that motion will be after it has become settled or steady.

Divide the whole fluid by concentric cylinders whose radii continually differ by $\delta r$. Then we may suppose the fluid between any two of these to solidify, and the circumstances of the case will not be in the least altered, provided we at the same time make $\delta r$ diminish without limit. Let us then consider the friction between any two of these solidified cylindrical elements. Let $\omega$ and $\omega+\delta \omega$ be the angular velocities of two consecutive cylinders, and let the radius of the surface of junction be $r$. It is manifest that the relative velocity of the two cylinders is 


$$
r \frac{d \omega}{d r} \delta r
$$

and therefore the friction which is proportional both to the velocity and the number of particles that rub against each other will be proportional to

$$
r^{2} \frac{d \omega}{d r} \delta r
$$

In order that the motion of any cylindrical element may not change, the friction on its two sides must be equal. Hence the expression

$$
r^{2} \frac{d \omega}{d r}
$$

must be the same for all values of $r$; call this value $-\alpha$; hence, integrating, we have

$$
\omega=\frac{\alpha}{r}+\beta
$$

where $\alpha$ and $\beta$ are constants depending on the angular velocities of the inner and outer cylinders. Supposing these to be $\omega_{1} \omega_{2}$, respectively, and $r_{1} r_{2}$ to be the radii, we have the two equations

$$
\left.\begin{array}{l}
\omega_{1}=\frac{\alpha}{r_{1}}+\beta \\
\omega_{2}=\frac{\alpha}{r_{2}}+\beta
\end{array}\right\}
$$

to find $\alpha$ and $\beta$.

In order that this state of motion may be "stable," it is necessary that the centrifugal force should increase from the centre to the circumference, unless the fluid be sufficiently tenacious to resist the change of motion that might follow the inequality of pressure. 
If the fluid be homogeneous, this condition requires that

$$
\omega^{2} r=\frac{\alpha^{2}}{r}+2 \alpha \beta+\beta^{2} r
$$

should increase with $r$. Hence its differential coefficient

$$
-\frac{\alpha^{2}}{r^{2}}+\beta^{2}
$$

must be always positive; that is,

$$
\beta>\frac{\alpha}{r_{1}} ;
$$

on substituting for $\alpha$ and $\beta$ their values, this gives

$$
\frac{\omega_{2}}{\omega_{1}}>\frac{1}{2}\left(1+\frac{r_{1}}{r_{2}}\right) \text {. }
$$

If there be added or taken away from the fluid and cylinders any constant quantity of angular velocity, the mutual attraction of the particles of fluid will not be changed, and therefore the relative motion will remain the same as before. If we impress on the whole system such a velocity as to make $\beta=o$, we get

$$
\omega=\frac{\alpha}{r} ;
$$

and this is the case considered by Newton. Unless the fluid be heterogeneous, this state of motion is unstable. Each particle of fluid has a greater tendency to fly from the centre than those beyond it, and hence if any circumstance occurs to alter the symmetry of the motion, the particles of those strata which have too great a centrifugal force will immediately fly from the centre, and the whole motion will be changed. This state of motion is like the case of a stratum of a heavy fluid resting on the surface of a lighter one. 
Bernoulli, in his dissertation, entitled " Nouvelles Pensées sur le Systême de Descartes avec la Maniere d'en déduire les Orbites et les Aphélies de Planètes," has made two objections to these investigations. First, that Newton in calculating the amount of the friction between two layers of fluid has not considered the pressure between those layers; and, secondly, that in calculating the effect of the friction, he has not taken into account the arm of the lever at which it acts. Bernoulli then attempted to show that the density, being supposed to vary with the distance, the motion in the vortex will agree with that given by Kepler's third law.

But D'Alembert* showed that this does not follow from Bernoulli's own equations, for it appears that he has, in integrating, omitted the lower limit; thus he considered

$$
\int_{0}^{\dot{x}} x^{m-1} d x=\frac{x^{m}}{m},
$$

which is true only when $m$ is positive, whereas he afterwards assumes $m=-\frac{1}{3}$. The first of Bernoulli's objections has been anticipated by Newton himself. In the scholium to the fifty-second proposition, he says, "The matter by its circular motion endeavours to recede from the axis of the vortex, and therefore presses all the matter that lies beyond. This pressure makes the attrition greater and the separation of the parts more difficult, and by consequence diminishes the fluidity of the matter." D'Alembert remarks that Mr. Musschenbroek in some very exact experiments, found that when the velocity is small, the friction was proportional to the velocity, and not to the pressure. That similar results have been obtained by later experimentalists is evident from what has been said in a previous chapter. 
Bernoulli's second objection has more force, according to our preceding investigation, the friction being

$$
r^{2} \frac{d \omega}{d r} \delta r
$$

and the arm of the lever at which this acts being $r$, we have

$$
r^{3} \frac{d \omega}{d r} \delta r
$$

to be constant for all values of $r$, this gives

$$
\omega=\frac{\alpha}{r^{2}}+\beta \text {. }
$$

If a sphere begin to rotate and communicate an angular velocity to the surrounding fluid, the inner parts of the vortex will move quicker than those without, and these by friction will be continually communicating velocity to those outer strata. The vortex created will, therefore, always grow larger and larger, and the motion of the sphere will be continuously transferred from the centre to the circumference, until it is swallowed up and lost in the boundless extent of space. Hence there must be some active principle which may tend to communicate velocity to the cylinder, otherwise it will move slower and slower and finally lose all its motion. If any motion had been communicated to the infinite fluid also, the friction of the fluid and cylinder will never cease to retard or accelerate that body until the whole fluid and cylinder revolve round with the same angular velocity. This, therefore, ought to be the state of the Cartesian vortex. But it is well known that the planets do not describe their orbits in the same time.

Newton next argues that even if we grant the existence of an active principle that will keep up the angular velo- 
city of the Sun, yet still it is impossible to explain the existing phenomena by a vortex. The third law of Kepler declares that the squares of the periodic times of the planets are as the cubes of their mean distances. But his preceding propositions have shown that this is not the case in a vortex. It is true that his reasoning is founded on two hypotheses; first, that the resistance arising from friction varies as the velocity; and, secondly, that the degree of fluidity is the same throughout the vortex. Let us, however, suppose that the friction varies as the $m$ th power of the velocity, and the frictional power of the several strata as the $n$th power of the distance. Then, according to Newton, we have

$$
\begin{gathered}
r^{2}\left(r \frac{d \omega}{d r}\right)^{m} \cdot r^{n}=\mathrm{constant} \\
\therefore \omega=\frac{\alpha}{r^{\frac{n+2}{m}}}+\beta .
\end{gathered}
$$

Hence, that Kepler's law may be true, we must have

$$
n+2=\frac{3}{2} m ;
$$

either, then, $m$ is greater than unity, or $n$ is negative. But New ton considered that if the resistance did not vary as the velocity, it would vary in a less ratio, that is, $m$ less than unity, and that if the parts of the fluid had various degrees of fluidity, those parts that had least fluidity would be heaviest, and would, therefore, be furthest from the centre, that is, $n$ positive; hence he thinks that the theory of vortices cannot be made to explain the third law of Kepler.

The motion of the vortex cannot be made to agree with the two first laws of Kepler. The planets move in ellipses which are so placed that their major axes are not parallel. To account for this Descartes supposed the vortices them- 
selves elliptical. The fluid between two elliptic curves of motion must always keep between them, and therefore the velocity must be greatest where the distance between the paths is least. But by Kepler's law the velocity is such that the areas described are proportional to the time. These two conclusions can be shown not to agree. As an example, let us take the three orbits of Venus, Earth, and Mars. "At the beginning of the sign of Virgo, where the aphelion of Mars is at present," says Newton, "the distance between the orbits of Mars and Venus is to the distance between the same orbits at the beginning of the sign of Pisces as 3 to 2. Therefore the matter of the vortex between those orbits ought to be swifter at the beginning of Pisces than at the beginning of Virgo in the ratio of $3: 2$. Therefore the velocity of the Earth at the beginning of Pisces should be to its velocity in the beginning of Virgo in the same ratio. But judging from the Sun's diurnal motion, we know that the Earth is swifter at the beginning of Virgo than at the beginning of Pisces. Hence the hypothesis of vortices is not reconcileable with astronomical phenomena."

If a body be carried round a vortex, it must have the same density as the fluid along its path. In that case it may be regarded as a piece of solidified fluid, and its centrifugal force is just balanced by the pressures on its two sides. But if the density be different, this balancing no longer takes place. The centrifugal force will be greater or less than the difference of pressures, according as the density of the body is greater or less than that of the fluid. Hence the body will recede from or approach to the centre according as its density is greater or less than that of the fluid. If the strata of fluid be not placed so that their densities increased with the distance, not only would they themselves be in an unstable state, but the globes also, if 
displaced, would not return to their former orbits. We should, therefore, expect those planets which are furthest from the sun to be the densest; but the contrary is the case. - Principia, prop. viii., book iii., cor. 4 .

The planets that have satellites were supposed by Descartes to be the centres of smaller vortices; but it has been already shown, that every vortex continually grows larger and larger, and thus every part of the fluid will be agitated with a motion resulting from the action of all the globes. Therefore the vortices will not be confined to any certain limits, but by degrees will run mutually into each other, and by the mutual action of the vortices on each other the globes will be perpetually moved from their places. They cannot possibly keep any certain positions among themselves unless some force restrains them. Forces are necessary, therefore, not only to keep the globes turning, but also to keep them in their places. 


\section{CHAPTER X.}

THEORY OF COMETS.

1. The Comets are planets moving in very eccentric conic sections.

2. To determine the particular orbit of a given comet.

(1.) The visit of a comet presents to us a great mystery. It comes unexpectedly, remains but a short time, often presenting a magnificent spectacle, and then disappears in a manner as wonderful as its appearance. It is one of those great problems that God has set before us. Many of the ancients regarded them * as simple meteors, sent from the Supreme Being as signs of his anger or prognostics of future events. Others, as the illustrious Seneca, had better views on the subject - "I do not follow the opinion of our philosophers; I do not consider the comets to be passing fires, but as one of the eternal works of nature." $\dagger$

Newton set about the solution of this great problem in his usual logical manner. The first point to be settled was whether they were simple meteors of our atmosphere or not. Their want of diurnal parallax is a convineing proof that they are at least further off than the moon. But how much further? We must have some idea of their distance to determine to what class of forces their

\footnotetext{
* Pingrè, Comet ; Bossut, Hist. de Math.

+ Seneca, Nat. Quæst. lib. vii.
} 
motions are subject. Comets are found to move more than ordinarily slow or swift according to the position of the earth. They have, in fact, an annual parallax. Therefore they belong to the region of the planets. This result Newton confirms by some considerations on the brightness of their heads. He proves that they shine by reflected light, and then comparing their light with that of the planets, he shows how much those persons are mistaken who remove the comets almost as far as the fixed stars.

If the comets move in the region of the planets, they must be subject to the attractions of the sun and the other planets. Neglecting for a first approximation these latter attractions compared with that of the sun, we know that the comet, being attracted towards the sun by a force which varies inversely as the square of the distance, must move in a conic section with the sun in one focus, and describe round it areas proportional to the times. This conic section may be either an ellipse, hyperbola, or parabola. In the former case the squares of their periodic times are as the cubes of their mean distances. This will furnish us with a complete test of the truth of our arguments. By observations made on the latitude and longitude of a comet, we can calculate its orbit with an accuracy depending on the degree of approximation to which we have carried the solution. We can then determine the distance and position of a comet at any moment. If these results agree with observation, there can be no doubt that our theory is correct. The difficulty of the problem is unfortunately as great as its importance. The comets move in such elongated ellipses, that, as a first approximation at least, we may consider their orbits as parabolas. But Newton speaks of the problem, even when thus simplified, as "Problema hocce longe difficillimum."

(2.) Newton proposed two ways of determining the 
orbit of a comit. One of these may be found in his little treatise De Systemate Mundi, and the other in the Third Book of his Principia. The first is by far the simplest of the two. The small part of the comet's path is considered as a straight line, the two parts of which are described by the comet with the velocity it had at the beginning of those parts. These velocities are assumed to be $V$ times that in a circle at the same distance. Let a first approximation to the distance of the comet from the earth at the time of the middle observation be supposed known, and let us represent it by the letter $x$. Newton then shows how to draw a straight line which will be divided by the directions in which the comet is seen at the times of the three observations in the ratio of the intervals between those observations. If this straight line, thus found, can be described in the given time with the velocity given by our assumed value of $V$, the value of $x$ that corresponds to $V$ has been found; if not, a second value of $x$ must be assumed; and then by an application of the rule of false, a new value may be determined, which is nearer the truth, and by repeating this process, we can arrive at any degree of accuracy. The value of $V$ being therefore supposed known, the velocity, position, and direction of motion of the comet are also known at a given time. By the First Book we can describe the orbit that a body projected in a given manner from a given point will describe round a given centre of force. Begin, then, with an approximate value of $V$, and construct the orbit. Make a fourth observation on the comet when at a great distance from its position at the three first observations. The longitude thus found should agree with that in the orbit just constructed. If not, a new value of $V$ must be found, and by the rule of false, the true value may be continually approximated to. 
The other method begins by assuming the orbit to be parabolic.

Three observations of a comet are supposed to be made at given times; then the positions of the earth $\tau, t, T$, being known, we can draw the directions of the straight lines $\tau A, t B, T C$, joining the comet and the earth at those

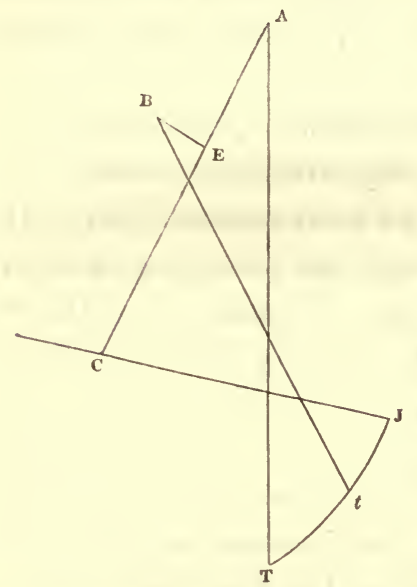
times. Suppose now that by some means a first approximation to the distance of the comet at the times of the second observation has been discovered, then we can nuark off a distance $t B$, and $B$ will be one point in the comet's orbit. Suppose also that from some properties of the parabola we can determine approximately, First, the length of the sagitta $B E$ drawn from the point $B$ of a comet's path in a known direction and cut off by the chord $A C$ joining the positions of the comet at the first and last observations; Secondly, the ratio in which the chord $A C$ is divided in the point $E$ : Thirdly, the length of the chord $A C$. Then, by means of the first two properties, we can construct the chord $A C$, and if its length be the same as that given by the third property, our assumption as to the value of $t B$ was correct. If not, by making several trials, and by applying the "rule of false," we can correct our assumed distance $t B$, and, therefore, ultimately obtain three points $A, B, C$ in the orbit. By a simple construction given in the First Book, a parabola can be described which passes through two points and has its focus in the centre of the sun. This will be the comet's orbit. 
Both these methods, it will be observed, require the distance of the comet at the time of the observation to be known at least approximately. The expedient* suggested was to consider a small portion of each orbit as rectilinear and described with uniform motion. Then four observations being made at moderate intervals of time, four straight lines are given, across which a straight line is to be cirawn so as to be cut in three parts in the same ratios of the intervals of time. This geometrical problem had been already solved by Wallis, Wren, and Newton, but its application to comets had never led to any satisfactory result. A little consideration will enable us to understand why. If the four straight lines pass through one point, the problem admits of more than one solution. Geometers call this a porismatic case. In fact, a whole family of straight lines can be drawn cutting the four straight lines in the required ratios. When the straight lines do not exactly meet in a point, only one straight line can be drawn; but, as might be expected, the construction to draw this line is such that any small error in the data will make a very great change in the cutting line. This, Boscovich remarks $\psi$, is exactly what occurs in the application to comets; the arcs of the two orbits, which are considered as straight lines, are necessarily small, and in this case the four straight lines can be shown to meet very nearly in one point.

Newton was not a man to be content with merely a general theory of comets; he at once reduced it to practice. He proceeded to try his method on the comet of 1680 . By scale and compass, in a figure in which the radius of the earth was $16 \frac{1}{3}$ inches, he determined the elements of the orbit. Halley afterwards again calculated these with

* Edin. Trans., vol. iii.

† Phil. Recent. a Benedicto Stay cum adnot. Boscovich, lib. ii. p. 345. 
greater accuracy by means of an arithmetical calculus. Moreover, observing that a remarkable comet had appeared four times at equal intervals of 575 years, he tried to find an elliptic orbit whose greater axis should be 138.2957 times the radius of the earth's orbit, so that a comet might revolve in it in 575 years. Taking this ellipse as the orbit of the comet, the observations from the beginning to the end were found to agree as perfectly with the computed places of the comet as the motions of the planets do with the theories from whence they are calculated; and this agreement clearly shows that it was one and the same comet that appeared all that time, and also that the orbit of that comet has been rightly defined. 

NOTES. 



\section{NOTE I.}

THE reasuning by which the law of density of a compressible fluid under the action of a central force was found may be extended to the determination of the conditions of equilibrium of any fluid under the action of any forces. This proposition is of course the foundation of the modern science of Hydrostatics, and may be investigated as follows-

To determine the conditions of the equilibrium of any fuids, acted on by any forces.

Choose any rectangular axes of reference, and take any small element whose edges are parallel to the axes and equal to $d x, d y, d z$ respectively. Let $x y z$ be the coordinates of one corner. Let X Y Z be the resolved parts of the accelerating forces acting on the element parallel to the axes, and let $\rho$ be the density of the fluid. If $p$ be the pressure referred to a unit of area at the corner $(x y z)$, the pressure on the side of the element parallel to the plane of $y z$ will be

$$
p d y d z,
$$

which by Law I. acts perpendicular to the face. The pressure on the opposite face will be

$$
\left(p+\frac{d p}{d x} d x\right) d y d z
$$

and therefore the pressure tending to move the element parallel to the axis of $x$ is

$$
-\frac{d p}{d x} d x d y d z
$$


But the moving force

$$
\rho \mathbf{X} d x d y d z
$$

tends to do the same, and since the element is in equilibrium, the sum of these two must be zero.

$$
\therefore \frac{d p}{d x}=\rho \mathrm{X} \quad-\quad-\quad-(1) \text {. }
$$

By Law II. the pressure referred to a unit of area on all the faces meeting at the corner $(x y z)$ are equal, and as similar reasoning applies to all the sides of the element, we shall have

$$
\begin{array}{lllll}
\frac{d p}{d y} & =\rho \mathrm{Y} & - & - & -
\end{array}
$$

These three equations are necessary and sufficient for equilibrium.

These equations may be put under a form which is very useful, and may sometimes be used independently of any axes of coordinates. For draw any curve in the fluid and consider an element $d s$ of its arc. Since no assumption has been made as to the axis of $x$, take it as tangent to the arc, and if $\mathrm{S}$ be the resolved part of the force along the tangent, the equation (1) shows that

$$
\frac{1}{\rho} \frac{d p}{d s}=\mathrm{S} \text {. }
$$

Reverting to the old position of the axes, the resolved parts of $\mathrm{X}$ Y Z along the tangent is

$$
\mathrm{X} \frac{d x}{d s}+\mathrm{Y} \frac{d y}{d s}+\mathrm{Z} \frac{d z}{d s} ;
$$

hence

$$
\frac{1}{\rho} d p=\mathrm{X} d x+\mathrm{Y} d y+\mathrm{Z} d z,
$$


an equation which we shall have frequent occasion to use in illustrating Newton's propositions.

Let us now consider some of the consequences of this proposition.

Consequence I. In order that there may be equilibrium, the forces and density of the fluid must be such that

$$
\rho \mathbf{X} d x+\rho \mathbf{Y} d y+\rho \mathbf{Z} d z
$$

is a perfect differential, that is, we must have

$$
\frac{d \rho \mathrm{X}}{d y}=\frac{d \rho \mathrm{Y}}{d x}, \frac{d \rho \mathrm{X}}{d z}=\frac{d \rho \mathrm{Z}}{d x}
$$

If we eliminate $\rho$ from these equations, we get

$$
\mathrm{X}\left(\frac{d \mathrm{Y}}{d z}-\frac{d \mathrm{Z}}{d y}\right)+\mathrm{Y}\left(\frac{d \mathrm{Z}}{d x}-\frac{d \mathrm{X}}{d x}\right)+\mathrm{Z}\left(\frac{d \mathrm{X}}{d y}-\frac{d \mathrm{Y}}{d x}\right)=0 .
$$

Hence unless this equation be satisfied there is no fluid which will be in equilibrium under the action of the forces. But if the equation be satisfied, the law of density must still be such as to satisfy one of the equations (5) (6). The only forces we meet with in nature are those which tend to fixed centres, and vary as some function of the distance from those centres. In all such cases the quantity

$$
\mathrm{X} d x+\mathrm{Y} d y+\mathrm{Z} d \boldsymbol{z}
$$

is a complete differential. For calling $r$ the distance of any point from one of the centres of force, and $\phi(r)$ one of the laws of attraction, we have

$$
\mathrm{X}=\Sigma \phi(r) \cdot \frac{x-a}{r}
$$

where $a$ is the abscissa of the centre of force, and similar expressions hold for $\mathrm{Y}$ and $\mathrm{Z}$. Hence

$\mathrm{X} d x+\mathrm{Y} d y+\mathrm{Z} d z=\Sigma \varphi(r) \frac{\overline{x-a} d x+\overline{y-b} d y+\overline{z-c} d z}{r}$

$$
=\Sigma \varphi(r) d r
$$


since $\left.\overline{x-a}\right|^{2}+\left.\overline{y-b}\right|^{2}+\overline{z-c}{ }^{2}=r^{2}$, and hence the above quantity is a perfect differential. The criterion is therefore satisfied, and it is therefore always possible to find a fluid that can be kept in equilibrium by the forces in nature.

Consequence II. Def. A surface along which the pressure is the same is called a level surface. It is also sometimes called a surface of equal pressure. A surface along which the density is the same is called a surface of equal density. Let us call the perfect differential

$$
\mathrm{X} d x+\mathrm{Y} d y+\mathrm{Z} d z=d \mathrm{P},
$$

then we have

$$
d p=\rho d \mathrm{P} \text {. }
$$

and this cannot exist, by a known theorem of the Differential Calculus, unless the three quantities $p, \rho, \mathrm{P}$ are functions, each of each. Hence the density is always a function of the pressure, and therefore

All level surfaces are also surfaces of equal density.

The differential equation to all level surfaces is

$$
\mathrm{X} d x+\mathrm{Y} d y+\mathrm{Z} d z=0,
$$

or, which is the same thing,

$$
\mathrm{P}=\text { constant. }
$$

Def. The bounding surface of any liquid exposed to any constant pressure, as for instance that exposed to the atmosphere, is called a free surface. All free surfaces are clearly level surfaces.

The resultant of the forces at any point is perpendicular to the surface of the level surface passing through that point.

Take the point as origin, and the tangent plane to the surface as the plane of $x y$, so that $d z=0$, and the axis of $z$ is normal. Along the surface we have

$$
\mathrm{X} d x+\mathrm{Y} d y+\mathrm{Z} d z=0
$$


or,

$$
\mathbf{X} d x+\mathbf{Y} d y=0 \text {; }
$$

but $d x$ and $d y$ are quite independent and can have any ratio

$$
\therefore \mathrm{X}=0, \quad \mathrm{Y}=0 \text {, }
$$

that is, $\mathrm{Z}$, the only force, acts along the normal.

Consequence III. If the fluid be elastic, Amontem has shown, by experiment, that there must be the relation

$$
p=k_{p}(1+\alpha \theta)
$$

between $p$, $\rho$, and $\theta$ the temperature, $k$, is a constant quantity depending on the nature of the gas, and $\alpha$ a numerical quantity $=\frac{1}{4 \frac{1}{1}}$, nearly. Then we have

$$
\begin{aligned}
d p & =\rho d \mathrm{P}, \\
\text { or } \frac{d p}{p} & =\frac{d \mathrm{P}}{k(1+\alpha \theta)}
\end{aligned}
$$

hence, as before, $p, \theta, \mathrm{P}$ are functions each of each, so that All level surfaces ure surfaces of equal temperature. 


\section{NOTE II.}

THe law of density of the several strata of the air, determined by Newton, is not, as we have seen, sufficiently accurate to be of any practical utility.

Nevertheless the expressions for the density in times of the height are not without their use. Thus they enable us to determine the heights of mountains by barometric observations. Let $p, \rho, \tau$ be the pressure, density, and temperature at an altitude $z$. Let $a$ be the radius of the earth, $g_{0}$ the force of gravity at the surface. Then the force of gravity at an altitude $z$ will be very nearly

$$
g=g_{0}\left(1-n \frac{z}{a}\right)
$$

where $n$ is some constant. If the upper station be in the air, the law of gravity shows us that

$$
n=2 \text {; }
$$

but if, as is usually the case, the upper station be on a mountain, the attraction of the elevated ground must not be neglected. In this case the value of $n$ will depend on the form of the mountain. It is found that

$$
n=\frac{5}{4}
$$

is sufficiently accurate for our present purpose. Retaining the general form of $g$, we have, as before, the two equations

$$
\left.\begin{array}{l}
\frac{d p}{\rho}=-g_{0}\left(1-n \cdot \frac{z}{a}\right) d z \\
p=x p(1+\alpha \tau)
\end{array}\right\}
$$


Hence, by eliminating $\rho$

$$
\frac{d p}{p}=-\frac{g_{0}}{x(1+\alpha \tau)} \cdot\left(1-n \frac{z}{a}\right) d z .
$$

Integrate this, on the supposition that $\tau$ is constant,

$$
\log \cdot p=-\frac{g_{0}}{x(1+a \tau)}\left(z-n \frac{z^{2}}{2 a}\right)+c .
$$

Let $h_{0}, h_{1}$ be the heights of the mercury in the barometer at the lower and upper stations, and $\tau_{0}, \tau_{1}$, the temperatures at those stations respectively. Therefore the above expression gives

$$
\log \cdot \frac{h_{0}}{h_{1}}=-\frac{g}{x(1+\alpha \tau)} \cdot\left(z-n \frac{z^{2}}{2 a}\right)
$$

The value of $\tau$ being constant is taken as the mean of the two observed values $\tau_{0}, \tau_{1}$. It is this fact that the decrease of temperature is found by actual observation at every application of the formula that renders the results so trustworthy. If we neglect $z^{2}$ the above leads to

$$
z=20177\left(1+\frac{\tau_{0}+\tau_{1}}{900}\right) \log \cdot \frac{h_{0}}{h_{1}}
$$

in yards. The logarithms are in this formula the ordinary tabular ones to base 10, and the temperatures are expressed in degrees above $32^{\circ} \mathrm{F}$.

If very great accuracy be required, a variety of small corrections for $x, \alpha, g_{0}$ are necessary. The correction for the variation of gravity with the height may be found by substituting the value of $z$ thus found in

$$
n \frac{z^{2}}{2 a}
$$

and this is to be added to the former result.

(2.) There is another very interesting application of these 
formulæ which we find in the second volume of Laplace's "Mécanique Celeste." We are enabled to determine in some measure the form of the atmosphere of the heavenly bodies. We have as yet neglected the effect of the centrifugal force; suppose the angular velocity to be $\omega, \theta$ the co-latitude of the particle of atmosphere under consideration. Then including this force in our equation we have

$$
\frac{d p}{\rho}=-\frac{\mu}{x^{2}} d x+\omega^{2} x \sin . \theta d(x \sin \cdot \theta),
$$

where the earth is considered to be a homogeneous sphere whose particles attract according to the law of the inverse square of the distance. Now along the free surface of the atmosphere $p$ is constant, and $. \cdot d p=0$; hence

$$
\begin{gathered}
-\frac{\mu}{x^{2}} d x+\omega^{2} x \sin . \theta d(x \sin . \theta)=0 \\
\therefore \text { const. }=\frac{\mu}{x}+\frac{\omega^{2}}{2} \cdot x^{2} \sin ^{2} \theta .
\end{gathered}
$$

This therefore is the equation to the surface of the atmosphere. Let us compare the polar and equatorial diameters. Call them $2 \mathrm{R}$ and $2 \mathrm{R}^{\prime}$. When $x=\mathrm{R}$ we have $\theta$, the co-latitude, a right angle; hence

$$
\frac{\mu}{\mathrm{R}}+\frac{1}{2} \omega^{2} \mathrm{R}^{2}=\text { const. }
$$

when $x=\mathbf{R}^{\prime}, \theta$ is nothing, hence

$$
\begin{gathered}
\frac{\mu}{\mathrm{R}^{\prime}}=\text { const. } \\
\therefore \frac{\mu}{\mathrm{R}}+\frac{1}{2} \omega^{2} \mathrm{R}^{2}=\frac{\mu}{\mathrm{R}^{\prime}} \\
=\frac{\mathrm{R}^{\prime}-\mathrm{R}}{\mathrm{R}}=\frac{\omega^{2}}{2 \mu} \cdot \mathrm{R}^{\prime 3} .
\end{gathered}
$$

Now at the equator the centrifugal force is less than gravity, that is 


$$
\begin{gathered}
\omega^{2} R^{\prime}<\frac{\mu}{R^{\prime 2}} \\
\therefore \frac{R^{\prime}-R}{R}<\frac{1}{2} \\
\therefore R^{\prime}<\frac{3}{2} R .
\end{gathered}
$$

Laplace deduces from this that the zodiacal light cannot be part of the Sun's atmosphere, for it has the form of a very flat lens, in which the polar diameter is far less than two-thirds the equatorial. Another sufficient reason is, that an atmosphere cannot extend beyond the orbit of a planet which describes its revolution in a time equal to the rotation of the Sun. Hence, as the Sun revolves in twenty-five days and a half, its atmosphere cannot extend so far as Mercury or Venus. We know that the zodiacal light extends much further. It is therefore not part of the Sun's atmosphere. The zodiacal light is a lenticularly shaped envelope which revolves round the Sun. Herschel ( $\$ 897$.) conjectures it to be no more than the denser part of that medium which resists the motion of comets, loaded perhaps with the actual materials of the tails of millions of those bodies, of which they have been stripped in their successive perihelion passages. It is an illuminated shower or tornado of stones. According to Professor Thomson, the inner parts of this tornado are always getting caught by the resistance of the Sun's atmosphere, and drawn to his mass by gravitation. They are always approaching the Sun, but very gradually, and he asserts that the mere fall of these aerolithes on the Sun is sufficient to account for the permanence of the Sun's heat. 


\section{NOTE III.}

THE whole calculation of Newton is founded on the supposition that the earth is homogeneous. Taking this for granted, it is possible to investigate without any long analysis the form of the earth. We cannot easily prove that the spheroid is the only form of equilibrium, but we shall show that it is at least one of the forms of equilibrium. That therefore if the earth had been originally fluid, it might have assumed a spheroidal form; or if not originally fluid, yet if created in this form that its several parts would have no tendency to break up.

A spheroidal mass of homogeneous fluid revolves round an axis with a uniform velocity; to determine if the equilibrium of the fluid be possible, and if so what is the ellipticity of the surface.

Let $a, b$ be the axes of the surface, $e$ and $\varepsilon$ the eccentricity and ellipticity of the surface, then by definition

$$
e^{2}=1-\left(\frac{b}{a}\right)^{2} \varepsilon=1-\frac{b}{a} .
$$

Let the axis of revolution $(b)$ be taken as the axis of $z$, and let the centre be the origin. Let $\omega$ be angular velocity, $\rho$ the density of the fluid. It is well known that the attractions of the spheroid on a particle whose co-ordinates are $x y z$ resolved parallel to those axes are respectively

$$
\begin{aligned}
& \frac{2 \pi \rho}{e^{3}}\left\{\sqrt{1-e^{2}} \sin ^{-1} e-e\left(1-e^{2}\right)\right\} x \\
& \frac{2 \pi \rho}{e^{3}}\left\{\sqrt{1-e^{2}} \sin ^{-1} e-e\left(1-e^{2}\right)\right\} y
\end{aligned}
$$




$$
\frac{2 \pi \rho}{e^{3}}\left\{e-\sqrt{1-e^{2}} \sin ^{-1} e\right\} z \text {. }
$$

For brevity's sake call the coefficients of $x, y, z, \mathrm{~A}, \mathrm{~A}, \mathrm{~B}$. Then, according to the notation of last chapter, we have

$$
\begin{aligned}
& \mathrm{X}=-\left(\mathrm{A}-\omega^{2}\right) x \\
& \mathrm{Y}=-\left(\mathrm{A}-\omega^{2}\right) y \\
& \mathrm{Z}=-\mathrm{B} z
\end{aligned}
$$

The equation to the surface is

$$
\begin{gathered}
\mathrm{X} d x+\mathrm{Y} d y+\mathrm{Z} d z=0 \\
\text { or }\left(\mathrm{A}-\omega^{2}\right) x d x+\left(\mathrm{A}-\omega^{2}\right) y d y+\mathrm{B} z d z=0
\end{gathered}
$$

integrating we have

$$
\frac{\mathrm{A}-\omega^{2}}{\mathrm{~B}}\left(x^{2}+y^{2}\right)+z^{2}=\text { constant },
$$

the equation to a spheroid; hence the equilibrium is possible. The eccentricity will be given by

$$
1-e^{2}=\frac{\mathrm{A}-\omega^{2}}{\mathrm{~B}}
$$

or by substitution

$$
\frac{\omega^{2}}{2 \pi \rho}+\frac{3\left(1-e^{2}\right)}{e^{2}}-\frac{\left(3-2 e^{2}\right) \sqrt{1-e^{2}}}{e^{3}} \sin ^{-1} e=0 .
$$

The quantity $\frac{3 \omega^{2}}{4 \pi \rho}$ is the same as

$$
\frac{\omega^{2} a}{\frac{4}{3} \pi \rho a}=\frac{\text { centrifugal force at equator }}{\text { equatorial gravity }}
$$

and this we usually denote by the letter $m$. Hence the equation to find $e$ becomes 


$$
-\frac{3\left(1-e^{2}\right)}{e^{2}}+\frac{\left(3-2 e^{2}\right) \sqrt{1-e^{2}}}{e^{3}} \sin ^{-1} e=\frac{2}{3} m .
$$

We have already seen that $m$ is nearly $\frac{1}{2} \frac{1}{0}$, whence we find

$$
e=\frac{1}{232} \text {. }
$$

If a curve be constructed of which the abscissa is $e$ and the ordinate the left hand side of the above equation, it will be found to resemble from $e=0$ to $e=1$, the line OE BC where $\mathrm{OC}=1$. Take $\mathrm{OG}=\frac{2}{3} m$, and draw $\mathrm{GEF}$ parallel

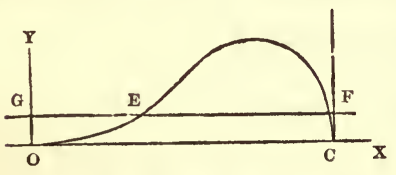
to the axis of $\mathrm{X}$, then we see that the curve cuts this straight line twice, and each of these points corresponds to a value of $e$ that will satisfy the above equation. It would appear that for the same value of $\omega$ there are two values of $e, \mathrm{GE}$ and $\mathrm{GF}$, which are consistent with equilibrium. But it does not follow that if a mass of fluid be set in motion with an angular velocity $\omega$ that it can take either of these forms. There is another condition to be satisfied. There is a certain principle in mechanics, called the conservation of areas, which teaches us that "if any number of bodies revolve round a centre, and are acted on only by their mutual attraction and by forces directed to the centre, the sum of the products of the mass of each by the projection on a given plane of the area which it describes round that centre bears a constant ratio to the time." Hence the fluid must take up such a form that this ratio shall be the same as that in the fluid as originally set in motion. It requires but little consideration to perceive that for two forms so different that one is nearly a sphere and the other excessively elliptical, it is impossible that the same angular velocity could sweep out the same areas in the same time.

This form of equilibrium of the earth is stable, for if the form were, by any chance, to become less spherical, by the principle of the conservation of areas, the angular 
velocity would decrease, and therefore the earth would return to its original form. So also, by similar reasoning, the same would occur if the earth were to become less elliptical.

If $\varepsilon$ be small, it is easy to see that the preceding equation leads us to

$$
\varepsilon=\frac{5}{4} m,
$$

since $2 e^{2}=\varepsilon$, when the powers of $e$ higher than the second are neglected. 


\section{NOTE IV.}

5. THE problem to determine the ellipticity of a planet considered as heterogeneous, is by no means an easy one. It certainly was beyond the powers of an age when the laws that govern the equilibrium of fluids were almost unknown. Newton determines the form of equilibrium from the condition that the weights of all columns of fluid, from the centre to the surface, must be equal. This, however, is not sufficient for equilibrium. Huygens added afterwards another condition, that the form of the surface must always cut perpendicularly the direction of the resultant force. But even these two conditions together are not sufficient. Clairaut (Figure de la Terre, Chap. III.) gave an instance in which, under a particular law of gravity, the particles of fluid could be so arranged that both these conditions were satisfied; yet he also showed that, so far from the fluid being in equilibrium, it was actually impossible for any fluid to rest in equilibrium under the action of these forces. It was Clairaut who first investigated all the necessary conditions of equilibrium, and showed that both the principles hitherto used were included in the one he proposed. His famous work, "Théorie de la Figure de la Terre," was published in 1743, and in it he applied his theory to determine the form of the earth considered as heterogeneous. Very little has been effected in this subject since his time. The form of the investigation has been changed, but all the results remain essentially the same. The form of the earth, whatever it may be, must consist of "level" strata of equal density, of which the surface is one. Clairaut assumes all these to be 
spheroids, having their minor axes in the same direction, but not necessarily of the same ellipticity. He then shows that when there is a certain relation between the density of any stratum and its ellipticity the fluid will be in equilibrium. Assuming that the density increases with the depth, it follows that the ellipticities must decrease from the surface to the centre, so that the strata are more and more nearly spherical the nearer they are to the centre of the earth. Suppose the earth to consist of a spheroidal nucleus formed of spheroidal strata of different densities, surrounded by a very thin layer of fluid (the sea), and suppose the laws of the density and the ellipticity of the strata to be any whatever, except that the ellipticity of the outermost stratum is the same as that of the thin layer of fluid upon it, then if $\mathrm{G}$ be the equatorial gravity, $g$ the gravity at latitude $\lambda, \varepsilon$ the ellipticity of the outermost stratum, $m$ the ratio of the centrifugal force at the equator to the equatorial gravity,

$$
\begin{aligned}
& g=\mathrm{G}\left(1+n \sin _{\circ}^{2} \lambda\right) \\
& n=\frac{5}{2} m-\varepsilon .
\end{aligned}
$$

This is a very remarkable proposition : the law of gravity along the surface of the earth is then quite independent of the law of density in the interior. It also furnishes us with a method of determining the ellipticity by observations on the force of gravity in different parts of the earth. It is usually called "Clairaut's Theorem."

If the earth was not originally fluid the strata of equal density may not have been spheroidal. But merely assuming that they differ but little from spheres, and that the surface is covered by a fluid in equilibrium, Laplace has shown that the changes in the force of gravity at the surface, and for all external points, is quite inde- 
pendent of the nature of the internal structure. There is a certain general connection between the form of the surface and the variation of gravity which he establishes on the above suppositions. This general connection has been lately demonstrated without making any hypothesis respecting the distribution of matter in the interior of the earth, but merely assuming the law of universal gravitation.

The investigations of Laplace lead to the same result as those of Clairaut, but there is this difference between their modes of reasoning. Clairaut assumes that the forms of the strata are spheroidal, and then shows that the whole will be in equilibrium. Laplace merely assumes that they differ but slightly from spheres, and then deduces from the condition of equilibrium that their forms are spheroidal. The solution of the question would in fact lead to a functional equation : we cannot write down the condition of equilibrium without knowing the attraction, and we cannot find the attraction without knowing the form of equilibrium. The analysis by which Laplace was enabled to prove the strata spheroidal is entirely his own invention. Its power is very great, and in many other investigations it has proved a useful engine of discovery.

6. We have seen that the earth cannot be homogeneous; we have also learnt from the investigations of Clairaut and Laplace that it consists of strata of different densities increasing from the circumference to the centre : it becomes an interesting question to determine this law of density. Legendre was the first who ascertained what is very probably the true law. But it is the more finished results of Laplace that we shall now consider. They are entirely built upon one assumption. We know that in gases and fluids the ratio of the change of pressure to the change in density is constant. But in solids and semi-fluid bodies it is more natural to suppose that this ratio increases with 
the density. The most simple assumption is to suppose that it varies as the density. Supposing this to be the truth it is not difficult to investigate the density of the strata. But it is an assumption, and must stand or fall according as its results agree with, or differ from, those of observation. Fortunately it enables us to integrate the equation connecting the density and ellipticity of any stratum, and thus the ellipticity of the external stratum furnishes us with a test of the truth of the law.

Taking for granted the truth of the law, a very simple calculation will give us the corresponding law of density. Let us consider the earth as a perfect sphere, and let us neglect the effect of the centrifugal force. The strata of equal density will then all be spheres. Let $\rho$ be the density of that stratum whose radius is $x$. Let $p$ be the pressure at that stratum referred to a unit of area.

We have first to find the attraction on a particle situated in the stratum whose radius is $r$. The attractions of all the external strata is manifestly nothing. To find the attractions of the internal ones, we have merely to suppose them concentrated into their common centre and attracting according to the usual law. This will manifestly give

$$
\frac{4 \pi \mu}{r^{2}} \int_{0}^{r} \rho x^{2} d x,
$$

where $\mu$ is the force of attraction of a unit of mass at a unit of distance. The law of fluid equilibrium will then give

$$
d p=-\rho \cdot \frac{4 \pi \mu}{r^{2}} \cdot \int_{0}^{r} \rho x^{2} d x \cdot d r
$$

Also we have by our assumption

$$
d p=x \rho \cdot d \rho-\ldots
$$

whence we get 


$$
\frac{x}{4 \pi \mu} \cdot \frac{d \rho}{d r}=-\frac{1}{r^{2}} \int_{0}^{r} \rho x^{2} d x
$$

or, which is the same thing,

$$
\frac{\kappa}{4 \pi \mu} \cdot\left(r \frac{\overline{d \rho r}}{d r}-\overline{\rho r}\right)=-\int_{0}^{r} \rho r^{2} d r
$$

differentiating,

$$
\begin{aligned}
& \frac{x}{4 \pi \mu} \cdot r \frac{d^{2} \overline{\rho r}}{d r^{2}}=-\rho r^{2} ; \\
& \therefore \frac{d^{2} \rho r}{d r^{2}}+\frac{4 \pi \mu}{x} \cdot \rho r^{2}=0 .
\end{aligned}
$$

Put $\frac{4 \pi \mu}{x}=q^{2}$ for the sake of brevity. We have now a differential equation to find $\rho r$. The integral is well known to be

$$
\rho r=\mathrm{A} \sin (q r+\mathrm{B}) \text {. }
$$

Now because the integral in equation (3.) has $r=0$ for its lower limit, it will be found on substitution that this value of $\rho r$ will not satisfy it unless $B=0$. Indeed, if $B$ were not zero, we would have the density infinite at the centre, which is manifestly impossible; for, no matter how great the pressure may be, it must still be finite.

We have then

$$
\rho=\mathrm{A} \frac{\sin q r}{r}
$$

This gives a density gradually decreasing from the centre, and therefore not contrary to what is a priori probable in the case of the earth.

The values of $\mathrm{A}$ and $q$ have yet to be determined. But 
the experiments of Cavendish and Maskelyne have revealed to us the mean density of the globe; and, supposing the density of the superficial stratum to be the same as that of granite, we have two equations to determine $\mathrm{A}$ and $q$. Working out these numerical calculations, we find that

$$
\rho=\frac{2 \mathrm{D} a}{r} \cdot \sin \left(\frac{5 \pi}{6} \cdot \frac{r}{a}\right),
$$

where $\mathrm{D}$ is the density of the superficial stratum.

There are two results which will serve to verify this law.

1. We know that there is a precession of the equinoxes, because the resultants of the attractions of the sun and moon do not pass through the centre of the earth. The position of the lines of action of these resultants manifestly depend on the law according to which the density of the several strata varies. This calculation has been made, and it is found that, taking the above law of density, the precession should amount to $51^{\prime \prime} \cdot 3566$. The observed precession is $50^{\prime \prime} \cdot 1$.

2. The ellipticity of the earth is caused by its rotation, and depends also on the law of density of its strata. The calculated result is $\varepsilon=\frac{1}{307 \cdot 313}$. The result of geodetic measures is $\varepsilon=\frac{1}{306}$.

It is upon the remarkable agreement of our supposition with observation in these two cases that our belief in the law is founded.

7. But though we have thus investigated the law of density of the strata, it is not to be therefore concluded that the earth is solid throughout; on the contrary, the rate at which the temperature increases with the depth is such that if continued for 25 miles, the heat would be sufficient, under a pressure of one atmosphere, to melt a stratum of granite. Is, then, the interior of the earth a vast mass of molten strata whose densities obey the law already investigated? and if so, what is the thickness of the crust? 
Mr. Hopkins has performed some very laborious calculations with a view of determining this question. $\mathrm{He}$ begins with some general remarks. There are two ways in which a body may cool, by conduction or convection. The earth being at first fluid, would begin to cool by convection. Now the temperature and pressure will both be greater at the centre than near the circumference. Because the temperature is greater at the centre, the body will solidify first at the outer parts, and the earth would become a crust containing a heterogeneous fluid. But because the pressure is greatest at the centre, the body will tend to solidify first at the centre, and thus on cooling it would become solid throughout. We cannot tell which is the predominating cause, and the investigation of the earth's refrigeration leaves the point uncertain. But there may be other tests whereby we can determine this question.

The precession of the equinoxes is caused by the attraction of the heavenly bodies on the ring of matter surrounding the earth's equator. One consequence of this attraction we have already seen to be the recession of the nodes in which it cuts the ecliptic. But this ring is fastened to the earth : its nodes cannot, therefore, recede as fast as they would do if the ring were left to itself. The earth is a heavy load which it has to pull round with it. The less this load the greater would be the precession. If the interior surface of the solid crust be spherical, then neglecting the friction between it and the interior fluid, the ring of matter surrounding the equator will only have to pull round with it the solid crust; the fluid will not turn with it. Hence the precession will be greater than if the earth were solid throughout.

But the interior surface of the crust cannot be supposed spherical; it is most probably spheroidal. Supposing it so, there will be pressures between its interior surface and the contained fluid, caused partly by the motion of the spheroid and partly by the tidal actions in the fluid caused 
by the attractions of the Moon and Sun and by the centrifugal force. These pressures must of course be taken into account.

It would be uninteresting to follow step by step the process of the investigation : they are very long, and I shall therefore confine myself to stating the results. $\mathrm{Mr}$. Hopkins * first considers the earth as a homogeneous spheroidal shell filled with a homogeneous fluid of equal density. The two surfaces of the shell are supposed to have equal ellipticities. On these suppositions he calculates the disturbing forces, forms the differential equations for the motion of the pole, integrates them, and by interpretation arrives at the following results.

“ 1 . The precession will be the same, whatever be the thickness of the shell as if the whole earth were homogeneous and solid.

"2. The lunar nutation will be the same as for the homogeneous spheroid to such a degree of approximation that the difference is inappreciable to observation.

" 3 . The solar nutation will be sensibly the same as for the homogeneous spheroid, unless the thickness of the shell be very nearly of a certain value, something less than one-fourth of the earth's radius, in which case the nutation might become much greater than for the solid spheroid.

" 4 . In addition to the above motions of precession and nutation, the pole of the earth would have a small circular motion, depending entirely on the internal fluidity. The radius of the circle thus described would be greatest when the thickness of the shell would be least, but the inequality thus produced would not for the smallest thickness of the shell exceed a quantity of the same order as the solar nutation, and for any but the most inconsiderable thickness of the shell be entirely inappreciable to observation."

Thus it appears that the effect of these pressures between 
the shell and the contained fluid is that the general effect is the same as if the whole were solid. This method therefore fails to tell us anything of the thickness of the crust. But when we proceed to consider the case in which both the solid shell and the inclosed fluid are of variable density, we arrive at a different result. The disturbing forces will of course depend on the law of density: taking the law which we have already investigated, we can calculate these forces and compare them with those obtained in the case of a homogeneous shell. The required alterations can then be made in differential equations of the motion of the pole. We can thus find the precession. : In order that the result thus found may agree with that found by observation, there must be a certain relation between the ellipticity of the internal surface of the solid part and the mean thickness of the crust. This internal surface is a surface of equal solidity. If we knew, then, what function the solidity of a body is of the temperature and pressure, we should be able to express the ellipticity of a surface of equal solidity as a function of the depth. By equating the two values thus found, we should have an equation to determine the thickness of the crust. But we do not know the law of the ellipticities of the surfaces of equal solidity. If heat did not affect solidification, they would be the same as the surfaces of equal density. If density did not affect solidification, they would be the same as the surfaces of equal temperature. But both are acting causes. Hence the ellipticity of a surface of equal solidity passing through any point must lie between those of the surfaces of equal density and equal temperature passing through the same point. The ellipticity of the former decrease, those of the latter increase, from the surface to the centre. Hence, in order that the numerical value of precession may be accounted for, it is sufficient that the thickness of the crust shall not be less than a certain value, found to be about one-fourth to one-fifth the radius.

This reasoning fails therefore to give more than an 
inferior limit to the thickness of the crust. We learn that it must exceed 1000 miles. Mr. Hopkins has lately undertaken a series of experiments with a view to ascertaining the temperature at which bodies liquefy under great pressures. An account of these was given at the meeting of the British Association. When finally completed they will throw great light on the thickness of the earth's crust.

8. It is not to be supposed that no measures were undertaken to discover by actual observation the ellipticity of the globe: such measures would be an excellent test of the truth of the theory. There are three methods by which this may be effected. The earliest of these is by measurement of the length of a degree in different latitudes. The length of a degree varies as the radius of curvature of the elliptic meridian, and therefore increases from the equator to the poles. Now when the ellipticity is given, we can express in terms of it the latitude and equatorial radius, the length of a degree at that place. By two measurements of degrees we get two equations, and therefore can eliminate the equatorial radius and find the ellipticity. Some of these measures were effected before Newton's time, and their results, as we have seen, were used by him in determining the ellipticity. In 1684 Cassini measured an arc of eight degrees,* and found, to the astonishment of every one, that the length of the degree shortened as he approached the poles. This was in direct opposition to Newton's theory. It was objected that the difference between the measures of two consecutive degrees was so small, that it was possible that the errors of observation might make that which was really the lesser appear to be the greater. To settle this doubt it was determined that three arcs should be measured. Godin, Bouguer and La Condamine were sent to Peru in 1735 to measure an

* Encyc. Met., "Figure of the Earth." 
arc near the equator, and in the succeeding year Maupertuis, Clairaut, Camus and Le Mounier went to the Gulf of Bothnia, while the French arc was measured by the Cassinis and Lacaille.

The result was decisive, that the degrees shortened from the poles to the equator. But the three values of the ellipticity thus determined were very different. The observations of Peru and France gave $\frac{1}{3} \frac{1}{3}$, those of Peru and Siveden $\frac{1}{2} \frac{1}{14}$, those of France and Sweden $\frac{1}{17}$, but on making some necessary corrections they gave $\frac{1}{1} \frac{1}{2}$. So great differences would seem to imply that the earth differed considerably from a spheroidal form. This, however, may be partly accounted for, because two of the measures were in very mountainous countries. A great many measures of degrees, both along arcs of the meridian of a parallel of latitude, have been since undertaken. It would detain us too long to consider them individually. The most probable value of the ellipticity as deduced from them is $e=\cdot 003352$.

The second method has already been alluded to. By Clairaut's theorem we can determine the force of gravity at any place in terms of the latitude, the eccentricity and the force of gravity at the equator. Two observations of the force of gravity will therefore enable us to determine the ellipticity. The force of gravity, as we shall show when we come to speak of the pendulum, may be determined by the use of that instrument. It may also be found by comparing the weight of a body with the strength of a spring, or indeed any force that does not vary with gravity. The former is the most accurate.

By a comparison of a great number of pendulum observations Airy has deduced $e=\cdot 003535$. In the article, "Figure of the Earth," in the "Encyclopædia Metropolitana," the Astronomer Royal has formed a table of seventynine pendulum observations. Thirty of these he has set apart as being " useless for the investigation of the earth's 
form." If $g$ be the force of gravity in latitude $\lambda$, then by Clairaut's theorem

$$
g=\mathrm{G}\left\{1+n \sin ^{2} \lambda\right\} .
$$

The values of $n$ and $\mathrm{G}$ that are found to suit best with the forty-nine "first-rate observations" are

$$
\begin{gathered}
n=\cdot 005133 \\
\mathrm{G}=\pi^{2} \times 39 \cdot 01677
\end{gathered}
$$

Now if $m$ be the ratio of the centrifugal force at the equator to equatorial gravity,

$$
n=\frac{5}{2} m-\varepsilon,
$$

whence since $m=\cdot 0034672$, the ellipticity is easily seen to be $\varepsilon=\cdot 003535$.

The above expression for $g$ enables us to find the force of gravity in any latitude. From a comparison of the results of the forty-nine observations with those given by this formula Airy has deduced.

1. That, cateris paribus, gravity is greater on islands than continents.

2. That in high north latitudes the formula gives too small, and about latitude $45^{\circ}$ too large a value of $g$; near the equator the errors are about equally balanced.

3. There is no reason to think gravity to be different in different longitudes, as the irregularities on different meridians do not appear greater than those at places near one another. Nor does it appear that there is any difference between the northern and southern hemispheres.

The observations were all reduced to the level of the sea by Dr. Young's rule, which makes an allowance for the attraction of the earth above the level of the sea. But it has been pointed out by Professor Stokes, in the "Transactions of the Cambridge Society" for 1849, that this rule does not take into account all the effects of the irregular distribution of land and sea. "Besides the attraction of the land lying immediately under a continental 
station, between it and the level of the sea, the more distant parts cause an increase in gravity, since the attraction they exert is not wholly horizontal, on account of the curvature of the earth. Again the horizontal attractions due to the neighbourhood of a continent would cause a plumb line to point slightly towards it, and since a level surface is everywhere perpendicular to the vertical, the level of the sea must be higher than it would be if the continent did not exist. The correction therefore reduces the observation to a point more distant from the centre of the earth than if the continent were away; and therefore on this account gravity is less on a continent than on an island. The investigation shows this latter effect more than counterbalances the former; so that, on the whole, gravity is greater on an island than on a continent."

It is also probable that the ellipticity deduced by Airy is a little too great, owing to the decided preponderance of oceanic stations in low latitudes among the group where the observations were taken. On looking at the expression for $g$, we see that, in consequence, the calculated values of gravity would be a little too small, particularly for places near the pole. This will enable us to show that Airy's second conclusion is a mere repetition of the first; for that in high north latitudes, the formula should give too small a result is no more than what we should expect; while about $45^{\circ}$, the places of observation being all continental, the formula naturally gives too large a result. Thus we are now enabled to account, at least in great measure, for the anomalies that Airy has noticed.

In considering the earth as an irregular figure, we must define what we mean by the ellipticity. Let $a$ be the mean radius of the earth, $r$ the radius of any point whose colatitude is $\theta$. Then since the earth is nearly a spheroid,

$$
\frac{r-a}{\frac{1}{3}-\cos ^{2} \theta}
$$

is very nearly constant. Its variations, as we go from 
place to place, are irregular, and always very small. The mean of all the values of the above fraction may be defined to be the ellipticity. We cannot observe directly the value of $r$. In practice, therefore, we replace this fraction by another which contains $g$ instead of $r$. If we could make a vast number of observations in all parts of the world, no further correction would be necessary than merely taking the mean of all the observed values of $\varepsilon_{\text {. }}$ But as all our observations are made on land, and are few in number, our errors may all tend in one direction: it is therefore necessary to reduce our results to the surface of the spheroid.

The third method of determining the ellipticity is founded upon astronomical observations. If the earth were a perfect sphere and homogeneous, the elliptic orbit of the moon would only be affected by the known disturbances of the other heavenly bodies. But the earth is neither spherical nor homogeneous. Hence arise other inequalities in the moon's motion, and conversely, when these are observed, they will enable us to discover the ellipticity of the earth. Without making any assumption as to the law of density, the theory of the "Figure of the Earth" enables us to find its attraction on the moon: substituting these in the equations of the moon's motion, we can deluce two inequalities. The chief of these is the inequality in latitude, and is about $8^{\prime \prime}$, hence we get $e=\cdot 003370$. The other is the inequality in longitude, and lies between $6^{\prime \prime} \cdot 8$ and $7^{\prime \prime}$, the former gives $e=\cdot 003360$, the latter $e=\cdot 003407$.

It is not difficult to see what will be the general nature of the more important inequality. The consequence of the attraction of the ring of matter surrounding the earth at the equator, will manifestly be to pull the moon nearer the equator. The chief disturbance will therefore be that produced by a small force acting on the moon perpendicular to the plane of her orbit, and tending towards the earth's equator. The effect of such a force is easily seen. 
Let $\Omega \mathrm{M}$ be plane of the lunar orbit when the moon is at M. And suppose that in the small time $\tau$ the moon, if undisturbed, would describe

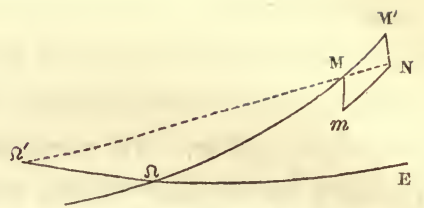
the arc $M^{\prime} M^{\prime}$. But if the moon were at rest at $M$, suppose that the disturbing force would pull it through half $\mathrm{M} m$ towards the plane of the equator $\Omega \mathrm{E}$. Then, by the second law of motion, if we complete the parallelogram $\mathrm{M} \mathrm{N}$, the true direction of the moon's motion at the end of the very small time $\tau$ will be $\mathrm{MN}$. That is, the orbit has been changed from $\Omega M^{\prime}$ to $\Omega^{\prime} M N$; the node $\Omega$ has receded, and the inclination of the orbit has been decreased. By similar reasoning it may be shown that if the moon had been approaching the equator in the direction $\mathrm{M} \Omega$ the node would have advanced, and the inclination would have increased.

During a quarter of a month the moon approaches the equator; during another quarter it recedes; and the forces being similar in each movement, the whole effect at the end of a month would be zero. But, owing to other disturbances, the node of the moon's orbit recedes nearly at a uniform rate along the ecliptic, while the inclination to the ecliptic remains nearly the same. Hence the inclination of the lunar orbit to the equator has changed in that half month; the disturbing forces in the two quartermonths, though they still tend to counteract each other, will not be quite equal in magnitude, hence a small residual effect will be left which soon mounts up till it becomes apparent.

By a little consideration of the preceding figure it will become apparent that the greater the angle of inclination at $\Omega$ the less will be the difference between the consecutive positions $\Omega \mathrm{M}, \Omega^{\prime} \mathrm{M}$ of the lunar orbit, $i, e$. the less the inclination $(i)$ to the equator will be changed. So that when by the motion of the nodes along the ecliptic $i$ is decreasing, the effect of the disturbing force will be greater 
in the second quarter-month than the first; that is, the effect will be greater while the moon is approaching the equator, than while it is receding from it, that is, by what precedes, the whole effect of the disturbing force is to lessen the diminution of $i$. Similarly, when $i$ is increasing, this increase is lessened by the action of the disturbing force. This is exactly what would take place if we neglected this disturbing cause and supposed the obliquity of the ecliptic to be less than it really was. Hence we arrive at this conclusion, which we shall state in the words of Laplace:-"The non-sphericity of the earth produces in the latitude of the moon but one sensible inequality. We can represent its effect by supposing the orbit of the moon instead of moving in the plane of the ecliptic with a constant inclination, to move with the same condition on a plane passing through the equinoxes between the ecliptic and equator. This inequality is well adapted for determining the ellipticity of the earth." 
NOTE V.

THE RESIstaNCE MADE TO BODIES MOVING IN FLUIDS DEDUCED FROM THE GENERAL PRINCIPLES OF DYNAMICS.

1. The Equation of motion.

a. The ordinary hydrodynamics.

$\beta$. How changed when "internal friction" is taken into account.

2. The ordinary law of resistance.

$\alpha$. How deduced from the equations of motion.

$\boldsymbol{\beta}$. The general results of experiments made since Newton's time compared with the law.

$\boldsymbol{\gamma}$. The resistance should be deduced from a rigorous solution of the equations of motion adapted to the case under consideration, case of the pendulum.

3. The resistance to a Pendulum.

a. Bessel's mode of expressing the resistance.

B. The careful experiments of Sabine, Baily, Coulomb, \&c.

$\gamma$. Poisson deduces the nature of the motion and the resistance from the ordinary Hydrodynamic equations.

$\delta$. On comparing the theory with experiment they are found not to agree.

€. Professor Stokes takes into account the effects of internal friction.

$\zeta$. The results agree with experiment.

4. The resistance to Floating Bodies.

$\alpha$. The phenomenon of emersion.

$\boldsymbol{\beta}$. Waves are excited in the Fluid.

$\gamma$. Strange variations of the resistance as the velocity changes.

(1.) After Newton the chief writers on Hydrodynamics were the Bernoullis, Maclaurin, and D'Alembert. The equations which the latter obtained are the foundation of modern Hydrodynamics. He had previously discovered a general principle whereby every question concerning the motion of bodies may be reduced to another corresponding one concerning their equilibrium. Thus then the science of Hydrodynamics may be reduced to that of Hydrostatics. The simplest case to which we can apply this principle is 
that in which the body is a single particle. It then leads us to the three laws of motion. When Newton said that a particle acted on by no external force will remain at rest or move in a straight line with a uniform velocity, he implied that there was no internal tendency in the particle to affect its state of rest or motion. D'Alembert extended this to any system of particles, and his principle asserts that the internal forces of a dynamical system are in equilibrium among themselves during the whole motion. It follows from this that the effective moving forces upon the molecules of a dynamical system, if their directions be reversed, will balance the external impressed forces. Let us apply this to the state of any fluid.

Let a small element be taken in any fluid in motion whose coordinates are $x, y, z$. Let $\rho$ be the density at this point and $p$ the pressure referred to a unit of area. And let $\mathrm{X}, \mathrm{Y}, \mathrm{Z}$ be the external impressed forces on the element. The effective accelerating forces will be

$$
\frac{d^{2} x}{d t^{2}}, \frac{d^{2} y}{d t^{2}}, \frac{d^{2} z}{d t^{2}}
$$

Hence the forces

$$
\rho \mathrm{X}-\rho \frac{d^{2} x}{d t^{2}}, \rho \mathrm{Y}-\rho \frac{d^{2} y}{d t^{2}}, \rho \mathrm{Z}-\rho \frac{d^{2} z}{d t^{2}}
$$

acting on the element $d x d y d z$, will, when all the elements are considered, balance each other. Hence from the equations of fluid equilibrium, we have

$$
\begin{gathered}
\frac{1}{\rho} \frac{d p}{d x}=\mathrm{X}-\frac{d^{2} x}{d t^{2}} \\
\frac{1}{\rho} \frac{d p}{d y}=\mathrm{Y}-\frac{d^{2} y}{d t^{2}}
\end{gathered}
$$




$$
\frac{1}{\rho} \frac{d p}{d z}=\mathrm{Z}-\frac{d^{2} z}{d t^{2}}
$$

These three equations are not, however, sufficient to determine the motion. For we have four quantities $x, y, z, p$ to determine in terms of $t$. A fourth equation is necessary. This D'Alembert supplied from the condition that any portion of the fluid, in passing from one place to another, preserves the same volume if incompressed, or dilates, according to a given law, if the fluid be elastic, in such a manner that the mass is unchanged. It is usual at present to derive this equation from a principle that in reality is only the above in another form. If $u, v, w$ be the velocities of the fluid at the point $x y z$ in the directions of the axes; then the result arrived at is

$$
\frac{d \overline{\rho u}}{d x}+\frac{d \overline{\rho v}}{d y}+\frac{d \overline{\rho w}}{d z}+\frac{d \rho}{d t}=0 .
$$

It will be observed that these equations do not make any assumption as to the molecular constitution of the fluid. All that is required is that the pressure, no matter how transmitted, shall be equal in all directions.

These equations are so complicated that hardly anything can be done with them. But there is one general case in which the equations are greatly simplified. This is when $\mathrm{X}, \mathrm{Y}, \mathrm{Z}, u, v, w$, are such that

$$
\begin{array}{lllll}
\mathrm{X} d x+\mathrm{Y} d y+\mathrm{Z} d z- & - & - & - & \text { (A) } \\
u d x+v d y+w d z- & - & - & - & \text { (B) }
\end{array}
$$

are perfect differentials, upon the supposition that the time is constant and the density either also constant or a function of the pressure. "It becomes then of the utmost consequence to inquire in what cases this supposition may be made. Now Lagrange enunciated two theorems by virtue of which, supposing them true, the supposition may be made in a great number of important cases; in fact, in 
nearly all those cases which it is most interesting to investigate. These are :-

" 1 . That (B) is approximately an exact differential when the motion is so small that squares and products of $u, v, w$, and their differential coefficients may be neglected.

“2. That (B) is accurately an exact differential at all times when it is so at one instant, and in particular when the motion begins from rest.

"It has been pointed out by Poisson that the first of these theorems is not true. In fact, the initial motion being arbitrary need not be such as to render (B) an exact differential.

"Lagrange's proof of the second theorem lies open to some objections." But it has received two perfectly satisfactory demonstrations.*

Supposing the motion to be such that we may put

$$
u d x+v d y+w d z=d \phi
$$

then it easily follows that

$$
\frac{d \phi}{d x}, \frac{d \phi}{d y}, \frac{d \phi}{d s}, \frac{d \phi}{d r}, \text { \&c. }
$$

represent the velocity parallel to axes and along the arc \&c. of the curve that the particle in question is describing.

At the time $t$ draw curves such that all the particles in them are at that moment moving along tangents to their respective curves. Let $s$ be the arc of any one of these

curves. The effective accelerating force will be $\frac{d^{2} s}{d t^{2}}$, and the impressed force

$$
\mathrm{X} \frac{d x}{d s}+\mathrm{Y} \frac{d y}{d s}+\mathrm{Z} \frac{d z}{d s} .
$$

* Report to the British Association, 1846, on the progress of Hydrodynamics by Professor Stokes. 
Hence, by D'Alembert's principle, and the formula for hydrostatical equilibrium,

$$
\frac{d p}{\rho}=\mathrm{X} d x+\mathrm{Y} d y+\mathrm{Z} d z-\frac{d^{2} s}{d t^{2}} d s .
$$

Let $\mathrm{V}$ be the velocity of the fluid, then since

$$
\begin{gathered}
\frac{d s}{d t}=\mathrm{V} \\
\therefore \frac{d^{2} s}{d t^{2}}=\mathrm{V} \frac{d \mathrm{~V}}{d s}+\frac{d \mathrm{~V}}{d t} \\
=\mathrm{V} \frac{d \mathrm{~V}}{d s}+\frac{d^{2} \varphi}{d s d t} .
\end{gathered}
$$

Hence, substituting and integrating,

$$
\int \frac{d p}{\rho}=\int(\mathrm{X} d x+\mathrm{Y} d y+\mathrm{Z} d z)-\frac{1}{2} \mathrm{~V}^{2}-\frac{d \phi}{d t}+\mathrm{C}
$$

an equation that will be necessary to us further on.

This integral is obtained by summing all the elements along any one curve, and $\mathrm{C}$ is therefore constant only along one curve, and may vary from curve to curve. To determine its variations we must have data given whereby we can know its value for all points along some surface cutting all the curves. Further it is manifest that these curves may change with the time; $\mathrm{C}$ is then a function of $t$.

The motion is said to be steady when the motion is always the same at the same point. Hence in this case neither $\phi$ nor $\mathrm{C}$ are functions of $t$. And the equation becomes

$$
\int \frac{d p}{\rho}=\int(\mathrm{X} d x+\mathrm{Y} d y+\mathrm{Z} d z)-\frac{1}{2} \mathrm{~V}^{2}
$$

The equation of continuity may also be greatly modified by the introduction of $\phi$. If the fluid be incompressible, we have 


$$
\frac{d^{2} \varphi}{d x^{2}}+\frac{d^{2} \varphi}{d y^{2}}+\frac{d^{2} \varphi}{d z^{2}}=0 .
$$

These equations are entirely founded on the principle that in fluids the pressure on an indefinitely small plane at any point is the same whatever be the position of the plane. This principle does not hold in viscous fluids. In $\mathrm{Hy}$ drostatics * if time be allowed to elapse, the substance changes until the pressure becomes equal in all directions. But in Hydrodynamics the motion of the fluid will have changed the relations of its particles before this time will have elapsed. If therefore we wish our equations to represent accurately the motions of ordinary fluids some account must be taken of these differences of pressure in different directions.

There are a variety of questions to whose solution the ordinary equations manifestly furnish no aid whatever. It will be sufficient to mention the motion of rivers in their beds, and the supply of water by a given pipe. There are few results that will not in some degree be affected by the viscosity of the fluid.

There have been many writers on this part of Hydrodynamics. Navier $\dagger$, Poisson $\ddagger$, Barré de Saint Venant $\S$, and Stokes $\|$ have investigated the equations of motion, but all on totally different principles. Two of these require us to consider the fluid as made up of ultimate particles; the others need no such supposition. But if the principles of their investigations are different, their results agree very well with each other. The equations arrived at are in the cases of a homogeneous incompressible fluid, and of an elastic fluid in which the change of density is small, those which would be derived from the common

* Mem. de l'Institut, vol. viii. p. 363.

† Mémoires de l'Académie des Sciences, vol. vi. p. 389.

¥ Journal de l'Ecole Polytechnique, xiii. cah. 20. p. 139.

$\S$ Comptes Rendus, vol. xvii. p. 1240.

|| Cambridge Philosophical Society, vol. viii. p. 287.; Report to British Association, 1846. 
equations by replacing $\frac{d p}{d x}$, the first by

$$
\begin{gathered}
\frac{d p}{d x}-\mathrm{A}\left(\frac{d^{2} u}{d x^{2}}+\frac{d^{2} u}{d y^{2}}+\frac{d^{2} u}{d z^{2}}\right) \\
-\mathrm{B} \frac{d}{d x}\left(\frac{d u}{d x}+\frac{d v}{d y}+\frac{d w}{d z}\right)
\end{gathered}
$$

and making similar changes in the second and third. The investigation of Professor Stokes makes $A=3 \mathrm{~B}$, and he has shown that this relation must exist even on Poisson's own principles. The coefficient $B$ is some constant depending on the "internal friction" of the fluid.

Such are the equations giving the motion in the interior of any mass of fluid. But we have still to consider the equation expressing the effect of boundaries to the fluid. In the case of ordinary hydrodynamics the condition is manifestly that along a free surface $p$ is constant, whereas along a surface bounding the fluid, the normal motion of the fluid must be the same as that of the surface. But when we consider the fluid as possessing internal friction, the last condition must be changed. The motion of the fluid in contact with the surface will clearly be in every way the same as that of the surface.

(2.) The whole theory of the resistance of bodies is included in the equations of fluid motion as enunciated above. It is therefore both interesting and important to deduce from them the law of resistance.

It is manifestly the same thing whether we conceive the body to be at rest, and the fluid to impinge on it, or the body to move with the same velocity through the fluid. Conceive then the fluid to be moving in a horizontal direction parallel to the axis of $x$, and let $z$ be the altitude of the surface of the water above the axis. Let a small plane be fixed in the axis of $x$, and perpendicular to it.

Following the usual notation, the equation of motion will be

$$
p=\mathrm{C}+g \rho z-\frac{1}{2} \rho v^{2} .
$$


This gives the variations of $p$ along any line of motion, and $\mathrm{C}$ may vary from one such line to another. By conceiving all these lines of motion cut by a plane perpendicular to the axis at a very great distance from the small plane, it will be seen that $\mathbf{C}$ is the same for all these lines of motion.

The theory supposes that the particles of the fluid as they approach the small plane move slower and slower, so that at last their velocity, when in contact with the plane, is so small, that it may be disregarded. The pressure in front is a statical pressure. On the other hand the particles in contact with the posterior part of the plane are supposed to move with the general velocity of the fluid in order to fill up the void that would be left by the retreating fluid. The pressure behind is a dynamical pressure. The difference between these two will be the resistance.

Hence the pressure in front is given by the formula

$$
p=\mathrm{C}+g \rho z^{\prime},
$$

that behind by the formula

$$
p^{\prime}=\mathrm{C}+g \rho z^{\prime}-\frac{1}{2} \rho v^{2} .
$$

The whole resistance will then be

$$
\mathrm{R}=\frac{1}{2} \rho v^{2},
$$

for every unit of area in the plane.

It is manifest that this contains a great deal of assumption in regard to the motion of the fluid. We have been trying to solve a question in Hydrodynamics without making use of all the equations, and each omitted equation has been replaced by an assumption. We should have taken the equation of continuity, and having solved it, we must adapt the solution to the conditions of the question, viz. that the velocity resolved parallel to the axis of $x$ is $r$, and that perpendicular is nothing at an 
infinite distance from the small plane, and that the normal velocity of the fluid over the surface of the plane is zero. Having thus found the motion, we can then substitute for $v$ in the equation above, and find the difference of pressures on the two parts of the plane. It is manifest that the solution in this case must contain discontinuous functions.

If the front of the plane be inclined to the direction of the motion of the fluid, one of the two suppositions on which the theory is built fails. We can no longer regard the velocity in front of the plane as zero. It is necessary to substitute another assumption. The theory supposes that the velocity in front of the plane will be equal to the general velocity of the fluid resolved in the direction of the plane. Let $\phi$ be the angle a normal to the plane makes with the general direction of motion. Then the velocity of the particles of the fluid in contact with the front will be $v \sin \varphi$, and the normal pressure will be given by

$$
p=\mathrm{C}+g \rho z-\frac{1}{2} \rho v^{2} \sin ^{2} \phi \text {. }
$$

Let us regard this plane as the oblique front of a cylinder moving in the direction of its axis. Let $B$ be the area of a perpendicular section, then the area $\mathrm{A}$ of the front will be $\frac{B}{\cos \varphi}$, and the normal pressure will therefore be $\frac{p \mathrm{~B}}{\cos \phi}$; resolving this along the axis, the pressure will become, B being supposed very small,

$$
p \mathrm{~B}=\left(\mathrm{C}+g \rho z-\frac{1}{2} \rho v^{2} \sin ^{2} \phi\right) \mathrm{B} .
$$

Let $\theta$ be the angle a normal to the oblique posterior plane of the cylinder makes with the general direction of the motion. Then, retaining the former supposition in 
regard to the motion of the fluid behind the plane, we shall have the normal pressure given as before by

$$
p^{\prime}=\mathrm{C}+g \rho z-\frac{1}{2} \rho v^{2} ;
$$

by reasoning as above, the resolved part of the pressure along the axis will be

$$
p^{\prime} \mathrm{B}=\left(\mathrm{C}+g \rho z-\frac{1}{2} \rho v^{2}\right) \mathrm{B} .
$$

The difference of these two pressures will be

$$
\begin{aligned}
\mathrm{R} & =\frac{1}{2} \rho v^{2} \cos ^{2} \phi \mathrm{B} \\
& =\frac{1}{2} \rho v^{2} \cos ^{3} \phi \mathrm{A} ;
\end{aligned}
$$

thus the resistance to a cylinder moving in the direction of the axis is independent of the inclination of the posterior end, and varies as the cube of the cosine of the inclination of the anterior end to the perpendicular section of the cylinder.

In determining the pressure on a curved surface, it is usual to consider each element of the front as the oblique end of a cylinder whose axis is parallel to the direction of motion; the corresponding element of the back of the body being the other oblique end of the cylinder. By integration, therefore, the whole pressure may be found. It also appears that the resistance depends only on the form of the front, and not at all on that of the back of the body. The suppositions on which this result is founded, are

1. That the velocity of the particles behind the body is the same as that of the general velocity of the fluid.

2. That the velocity along any element of the front is equal to the resolved part of the general velocity of the fluid along the plane of the element.

Throughout these investigations the motion of the fluid has been supposed steady. No applications of these results to any other case can be regarded otherwise than as an approximation. The relative velocity of the body 
and fluid must therefore be constant, or, at least, vary slowly.

If the fluid also be not boundless, but contained within rigid or flexible surfaces, as the body moves on, these may have some effect in disturbing the steadiness of the motion.

The fluid has been supposed incompressible. In applying the law to the case of a compressible fluid, we are omitting all consideration of the condensation in front and rarefaction of the fluid behind the body, and also of the vis viva lost by the waves that would be propagated in all directions round the body. These cannot be said to be unimportant.

And even if the fluid be incompressible, yet if the body be floating on its surface, the theory takes no account of the heaping up of the fluid in front of the vessel, nor the partial lowering behind, nor the consequent waves propagated in all directions round the vessel.

Having viewed the various considerations which have led to the ordinary law that Resistance varies as the square of velocity, it remains to compare this law with the results of experiment. This is the Lydian touchstone by which the true gold of any theory is discovered. To consider in detail all these would be at once uninteresting and unprofitable. We shall proceed to sum up the various results arrived at, and arrange them under the heads of the theory. We shall only mention a few cases* to show the application of the rules.

1. The resistance is only found to vary as the square of the velocity when the body is wholly immersed, the depth of the fuid not inconsiderable, and the velocity neither very small nor very large.

Coulomb found that for very small velocities the resistance varied as the velocity; the part depending on the square being inconsiderable.

- For some of these references we are indebted to the excellent article on Resistances by Sir D. Brewster, in the Encyc. Brit, 
The experiments ordered to be undertaken by the French Academy, in the last century, with velocities not greater than $2 \frac{1}{2}$ miles per hour ( $7 \cdot 6$ feet per second) showed that the resistance to floating bodies varied as the square of the velocity.

Hutton found that for low velocities in air the resistance varied as the square; but as the velocity increased to several hundred feet per second, the resistance varied as a power of the velocity greater than $2 \frac{1}{10}$. But as the velocity still further increased, the error of the ordinary law, though always very great, became slightly less.

Beaufoy. If the velocity exceed two miles per hour, or 6.2 feet per second, the power of the velocity is greater than 2; but this power decreases, and is less than 2 , if the velocity be greater than eight miles per hour.

Speaking of this law of resistance, Robins says_-"This rule, though pretty near the truth when the velocities are confined within certain limits, is excessively erroneous when applied to military projectiles, where such resistances often occur as could scarcely be effected on the commonly received principles, even by a treble augmentation of its density." The actual resistances were found to exceed prodigiously those calculated by the theory, when the velocity exceeded 1200 feet a second, which is the velocity with which air rushes into a vacuum.

Scott Russell found that in ships, as the velocity increased, the resistance increased in a much higher ratio than the square of the velocity, and reached a maximum, after which it actually decreased as the velocity increased.

Beaufoy found that bodies are more resisted just under the surface than at the depth of six feet.

Borda found a similar result.

2. The resistance to a plane does not vary as the surface, but increases in a quicker ratio.

Borda found that when the surfaces were as $9,16,36$, 81 inches, the resistances were as $9,17 \cdot 535,42 \cdot 750$, $104 \cdot 737$.

Nevertheless, the results of many experiments show that A 42 
the error made by assuming the resistance to vary as the surface is not very great, unless one of the surfaces is many times larger than the other. Thus the French experimentalists found this law in tolerable accordance with their results.

3. The resistance on an oblique plane does not vary as square of sine of angle the plane makes with the direction of motion.

The French Academy experiments were made with vessels with wedge-like prows of various angles. If $x$ be half the angle at the prow, the formula

$$
\mathrm{R}=\mathrm{P} \cdot \sin ^{2} x+3 \cdot 153\left(\frac{90-x}{6}\right)^{3 \cdot 25}
$$

represented the resistance so exactly that the error was always less than $\frac{7}{1} \frac{7}{0}$.

Hutton found that the formula

$$
\mathrm{R}=\cdot 84(\sin \theta)^{1 \cdot 84} \cos ^{2 \theta}
$$

represented the resistance.

Vince found the resistance did not vary as any constant power of the sine. Various other formulæ have also been given by Du Buat, Dr. Young, Eytelwein, \&c.

In the experiments of the French Academy, theory gave a result for an angle of $12^{\circ}$ only one fortieth of the truth. In some experiments by Vince for an angle of $10^{\circ}$, the resolved part of the resistance in the direction of motion was ten times that given by theory.

4. The changes of resistance calculated by the theory caused by changes of form are erroneous. The modification of resistance on any element produced by the near presence of others cannot be neglected.

If the formula of the experimentalists of the French Academy be employed to calculate the resistance on curvilinear surfaces the results are altogether erroneous.

Robbins found that the resistance on a sphere in air was 2.27 times the resistance on its great circle. Borda found $2 \cdot 45$. Hutton found $2 \cdot 4$. The ordinary law gives 2 . 
The second series of Bossut's experiments showed that the resistance in narrow or shallow canals was greater than in an indefinite fluid.

$\mathrm{Du}$ Buat deduced that the ratio of the resistance $\mathrm{R}$ in a narrow canal to the resistance $\mathrm{R}^{\prime}$ in an indefinite fluid was

$$
\frac{\mathrm{R}}{\mathrm{R}^{\prime}}=\frac{8 \cdot 64}{\frac{\mathrm{C}}{\mathrm{B}}+2}
$$

where $\mathrm{C}$ is the area of a section of the canal, B the area of a section of the vessel.

So many assumptions are made in the ordinary proof that the resistance varies as the square of the velocity, that it is no wonder if the law is found to be at variance with experiment. We must proceed in a different manner if we wish to deduce the true law of resistance. The difficulty of such an investigation is evident. Nevertheless, by the skill of some eminent mathematicians, some progress has been made. But the problem in its widest form has not been solved. For such particular cases as are most needed in practice, approximate solutions have been found. One of these, and the most important, is the pendulum. The innumerable applications of this instrument make its utility immense. Its very delicacy renders it a very efficient test of our theories of fluid motion. The resistance opposed to the uniform motion of bodies of certain forms have also been investigated.

(3.) In the year $1826 \mathrm{~F}$. W. Bessel published in the Royal Academy of Sciences at Berlin a new method of determining the length of the seconds' pendulum. In the course of it he pointed out an error in the old reduction to a vacuum. He states* that "if we denote by $m$ the mass of a body moving through a fluid, and by $m^{\prime}$ the mass displaced, the accelerating force acting on the body has, since the time

* Baily on the Correction of a Pendulum. 
of Newton, been considered equal to $\frac{m-m^{\prime}}{m}$. This formula is founded on the presumption that the moving force which the body undergoes, and which is denoted by $m-m^{\prime}$ is confined to the mass $m$. But it must be distributed not only over the moving body, but on all the particles of fluid set in motion by that body, and consequently the denominator of that expression denoting the accelerating force must necessarily be greater than $m$. From some general mathematical considerations he concludes that a fluid of very small density surrounding a pendulum has no other influence on the duration of the vibrations than that it diminishes its gravity and increases the moment of inertia."

The effect of the resistance of the air is then the same as if that air was removed and a mass of air, equal to $x$ times "the fluid displaced "was attached to the centre of gravity of the fluid displaced, which increases the inertia of the whole without affecting its gravity. The effect of the buoyancy of the air is the same as if a weight of air equal to that of the fluid displaced were removed from the centre of gravity of the fluid displaced, affecting the gravity but not the inertia. Let $l$ and $\gamma$ be the distance of the centres of gravity of the pendulum and air displaced from the axis of suspension, $\delta$ the ratio of the density of the fluid to the mean density of the pendulum, and let $i$ be the radius of gyration of the pendulum about an axis through its centre of gravity parallel to the axis of suspension. Then by merely uniting the equation of motion of the pendulum, it becomes evident that a pendulum affected as above stated will oscillate in exactly the same manner that a simple pendulum whose length is $\lambda$ does in vacuo, where

$$
\lambda=\frac{l^{2}+i^{2}+\gamma^{2} \cdot \delta \cdot x}{l-\delta \gamma} .
$$

This manner of expressing the result will be presently 
found useful. The whole object of the inrestigation is the determination of $x$.

This mode of viewing the resistance to the motion of a pendulum, was a great improvement. But Bessel was not the first discoverer, though he was the first who called the attention of the public to it. The chevalier De Buat had treated of this very property of the pendulum in his Principes d'Hydraulique published in 1786, fifty years before. "And it is not a little remarkable that these important and conclusive experiments of De Buat, which were made by the order and at the expense of the French Government, which were examined at the request of the Minister of War by the Royal Academy of Sciences at Paris, and by them favourably reported on, which were first published in the year 1786 and excited so much interest that they led to the subject for the prize essay proposed by the Academy in the following year, should have been so completely lost sight of and forgotten that no one should have had the least remembrance of the additional correction for the pendulum pointed out by M. de Bruat, and until the rediscovery of this principle by Bessel no one should have thought of verifying the suspicion of Newton, that such an effect was probable."-Baily on the Correction of a Pendulum, Phil. Trans. 1832.

A variety of experiments have lately been made to determine the resistance experienced by the pendulum as it makes its small oscillations in the resisting medium. One obvious method is to swing a pendulum in air and vacuo, and note the number of oscillations made in any given time. Recurring to our value of $\lambda$, it is easy to see that if $\mathrm{N}$ be the number of oscillations in vacuo, and $\mathrm{N}^{\prime \prime}$ the number in air in any given time, that

$$
\frac{\mathrm{N}^{\prime \prime}-\mathrm{N}}{\mathrm{N}}=\frac{\sqrt{\frac{l^{2}+i^{2}+\gamma^{2} \delta x}{l-\gamma^{\delta}}}-\sqrt{\frac{l^{2}+i^{2}}{l}}}{\sqrt{\frac{l^{2}+i^{2}}{l}}}
$$


By experiment, the value of the left hand side could be found, and therefore, by the solution of an equation, that of $x$. In making these experiments, the necessary corrections to $l, \delta, \& c$. due to heat and other causes must not be forgotten. It is not our office at present to consider these.

If $\mathrm{N}^{\prime}$ be the number of oscillations made in air as calculated on the old theory, then the value of $\frac{\mathrm{N}^{\prime}-\mathrm{N}}{\mathrm{N}}$ may be found from the above by writing $x=0$. And thence the value of $\frac{N^{\prime \prime}-N}{N^{\prime}-N}$ the number of times the old correction must be multiplied to get the new. This ratio we shall call $n$. By regarding $\delta$ as small, and expanding, we get

$$
\left.\begin{array}{c}
\frac{\mathrm{N}^{\prime \prime}-\mathrm{N}}{\mathrm{N}}=\frac{1}{2}\left(1+\frac{\gamma l}{l^{2}+i^{2}} x\right) \frac{\gamma}{l} \delta \\
n=1+\frac{\gamma l}{l^{2}+i^{2}} \cdot x
\end{array}\right\} .
$$

Now usually $\gamma$ is very nearly equal to $l$, and $\frac{i}{l}$ is very small; hence

$$
\left.\begin{array}{rl}
\frac{\mathrm{N}^{\prime \prime}-\mathrm{N}}{\mathrm{N}} & =(1+x) \delta \\
n & =1+x
\end{array}\right\} \text { nearly. }
$$

This is the method that has been adopted by Sabine and Baily. Their memoirs will be found in the Phil. Trans. for 1829 and 1832. The former experimented chiefly on the effects of different media and of changes in the external circumstances; the latter on the different resistances experienced by different forms of bodies.

Coulomb, in his experiments, adopted a different method; he made a small plane perform small oscillations in 
a fluid round a vertical axis, perpendicular to its plane, and passing through its centre. The force by which this was effected was the force of Torsion. His published account is in the Mem. de l'Institut, vol. iii. The mode of experimenting was adapted for the measurement of the decrements of the arcs, but not suited to the accurate determination of the effect of the fluid on the time of vibration.

The results of these experiments may be arranged as follows :-

1. That the effect of the resistance of the air is not insensible.-Du Buat, Sabine, Baily.

2. The value of $n$ does not depend on the density of the body, but only on the extent and form of surface. The body might even be hollow.-Baily.

3. Spheres. The value of $n$ is a little greater for small than large spheres.-Du Buat, Baily. But on the whole, $x$ is not subject to very great variations, and is nearly equal to $\cdot 7$ or $\cdot 8$. - Baily.

4. Cylinders. The value of $n$ varies according to the face opposed to the resistance, being greatest when the flat side is in front. In this case the height or thickness had considerable effect; the less this is, the greater is n.Baily.

5. Pendulum rod. The thinner the rod the greater is n.-Du Buat, Bessel, Baily.

6. Discs. Flat sides being opposed to the motion. The quantity of air dragged was found to be $149 d^{3}$, while that by a sphere was $\cdot 123 d^{3}$ grains troy, where $d$ is the diameter in inches. A disc, therefore, drags more than a sphere of equal diameter. When placed edgeways they dragged some air.-Baily.

7. Nature of the surface. On covering Coulomb's disc with a thin coating of tallow, the resistance was found to be the same as before, and even when the tallow was sprinkled with powdered sandstone by means of a sieve, the augmentation of resistance was "a peine sensible." 
8. Motion of the air next the pendulum. " On attaching a piece of gold leaf to the bottom of a pendulum, so as to stick out in a direction perpendicular to the surface, and then setting the pendulum in motion, Sir James South found that the gold leaf retained its perpendicular position, just as if the pendulum had been at rest, and it was not until the gold leaf carried by the pendulum had been removed to some distance from the surface that it began to lag behind."-Stokes's Cam. Trans., vol. ix.

9. When the air is reduced to half its usual density, the value of $x$ was found to be nearly the same; so that only half as much air was dragged.-One experiment of Sabine.

10. When hydrogen was substituted for air, the effects on the time was not proportional to the density. Air is thirteen times as dense as hydrogen, but the retardations measured by the number of vibrations were as $5 \frac{1}{4}$ to 1 . -Sabine.

11. A pendulum vibrating within glasses makes nearly the same number of vibrations as if the glasses were absent. The mean difference of three experiments of Sabine was $\cdot 007$ per diem.

12. The resistance to small velocities varies partly as the velocity and partly as the square of the velocity.-Coulomb.

Poisson has calculated from theory the exact effect of the resistance of the air on the motion of the pendulum. His memoir will be found in the third volume of Mém. de l'Institut, 1831. He supposes the oscillations of the pendulum very small, and as a consequence, those also of the fluid are very small. The equations of motion of the air, therefore, reduce themselves to the usual oquations for the propagation of sound. But the arbitrary functions are to be determined by the condition that the velocities of the molecules of air in contact with the pendulum are always the same, when resolved along the normal to the surface, as the velocity of that part of pendulum resolved in the same direction. The motion of 
the pendulum, after allowing for the buoyancy of the air, is affected by two causes, - the variations of pressure over its surface, and the friction of the air along its surface. This friction is assumed to be proportional to the difference of velocities of the air and surface resolved in any direction along the surface. He now forms the differential equations of the motion of the fluid and pendulum, but finds that without some further limitation they cannot be solved.

In the case, however, in which the surface of the pendulum is spherical, and the rod so thin that we may neglect the action between it and the air, the equations can be integrated, and the motion both of the fluid and sphere found. The motion is supposed to be given to the body by moving the pendulum very slightly from its position of rest, and then leaving it to the action of gravity, without impressing on it any velocity. At the commencement of the motion the whole air is supposed to be at rest-homogeneous and boundless in all directions.

The motion of the spherical pendulum is found to be the same as that of a simple pendulum of a certain length $\lambda$ oscillating in a medium resisting as the velocity. But it is to be remarked that this resistance is found to arise entirely from the friction of the air against the sphere. The assumption that this friction exists is directly contrary to the usual theory of Hydrodynamics. The very equations of motion are founded on the supposition that it does not exist, and therefore takes no account of the equal and opposite friction of the sphere on the fluid. In testing the theory we must omit this resistance, and the motion is therefore the same as that of a simple pendulum oscillating in vacuo. Theory, therefore, gives the arc constant. The value of $\lambda$ thus found is

$$
\lambda=\frac{l^{2}+i^{2}+\frac{1}{2} \gamma^{2} . \delta}{l-\gamma^{\delta}}
$$

comparing this with our former value of $\lambda$, we see that 


$$
x=\frac{1}{2} \text {. }
$$

The motion of the fluid is propagated in all directions with a.velocity equal to that of sound, and consists for each molecule of two sorts of vibrations; one whose vibrations are very rapid, the time being $\pi \frac{c}{a}$ sec, where $c$ is the radius of the sphere, and $a$ the velocity of propagation of sound. This will produce a sharp note, but insensible to the ear, because the amplitude of the vibrations containing the exponential $\varepsilon^{-\frac{\alpha t}{c}}$ rapidly decrease. The time of vibration of the other sort is equal to that of the pendulum, and its magnitude decreases successively according to the same law that those of the pendulum follow. These also will be insensible to the ear.

We have now to test the correctness of the theory by a brief comparison of its results with those of experiments. Recur to our list of results.

Theory teaches us that the resistance of the air is not insensible; that the value of $x$ does not depend on the interior structure of the body, and it explains why $x$ does not alter much, being a fraction depending on the form of the body and the external circumstances of the fluid; it enables us even to approximate to its true value. But it does not explain why $x$ is greater for small than large spheres, and the very principles of the theory in regard to the motion of the fluid in contact with the body is in opposition to certain experiments. Theory teaches also that the effect of the fluid is not a resisting force varying as some function of the velocity, but that its effect is simply to increase the inertia of the body, and thus leaves the arc of vibration constant, which is contrary to the commonest observations. Neither does the theory explain the difference between the resistances in hydrogen and air.

The two important points of the motion are the time and the length of the arc of vibration. Thus while the ordinary theory of Hydrodynamics agrees in the main 
with observation in regard to the time, it does not explain the minuter variations of the time, and totally fails to account for the successive diminutions of the arcs of vibration.

It becomes necessary to consider the effect of the internal friction of the fluid. This has been accomplished by Professor Stokes. The equations, however, in this case become so complicated, that it requires a very long: analysis to obtain the motion even of so simple a body as a sphere. We shall therefore merely state the results arrived at. The sphere is suspended by a fine wire, the length of which is much greater than the radius of the sphere. The resistance both to the sphere and the cylindrical wire have to be discussed. The motions are considered very small, so that by some obvious reductions the problem is reduced to the two following.

The centre of a sphere performs small periodic oscillations "along a right line in a boundless fluid, the sphere itself having a motion of translation only. Find the motion of the fluid.

An infinite cylinder performs small oscillations in a fluid in a straight line perpendicular to its axis. To find the motion of the fluid.

Let $\xi$ be the abscissa of the centre of the sphere at any time $t$. Let $\mathrm{T}$ be the time of one of its small oscillations from rest to rest, $a$ its radius, $m$ its mass. Let $m^{\prime}$ be the mass of the fluid displaced, $\rho$ its density, and $\mu$ some constant depending on the internal friction of the fluid. Then put

$$
\begin{array}{cc}
n=\frac{\pi}{\mathrm{T}}, & \nu=\sqrt{\frac{n \rho}{2 \mu}}, \\
=\frac{1}{2}+\frac{9}{4 \nu a}, & x^{\prime}=\frac{9}{4 \nu a}\left(1+\frac{1}{\nu a}\right) .
\end{array}
$$

Then the resistance on the sphere is 


$$
\mathrm{R}=-x m^{\prime} \frac{d^{2} \xi}{d t^{2}}-x^{\prime} m^{\prime} n \frac{d \xi}{d t} .
$$

The motion will therefore be the same as if the sphere were resisted by a force $x^{\prime} m^{\prime} n$ multiplied by the velocity, and a mass $x m^{\prime}$ were added to its centre, increasing the inertia without affecting the weight.

We are now enabled to account for many of our experimental results, that is, such of them as relate to spheres. We see that neither $x$ nor $x^{\prime}$ depend on the density of the sphere, but only on the volume; that both are greater for small than large spheres. The resistance also is independent of the roughness of the surface. One experiment of Sabine showed that $x$ remained the same when $\rho$ was reduced one half; this would seem to show that for the same fluid, at the same temperature, the value of $\mu$, the coefficient of the friction, varies as the density. But since the value of $x$ was not the same as before, when hydrogen was substituted for air, we see that in different media $\mu$ depends on something else besides the density.

We may apply these conclusions to the pendulum, and obtain results which we may test by experiments. The effect of the term depending on $x$ will clearly be to alter the time of the vibration, but not the arc of oscillation; the term depending on $x^{\prime}$ (being multiplied by the small factor $\frac{m^{\prime}}{m}$, whose square may be neglected) will not affect the time of the vibration, but will decrease the arc continually, so that the successive arcs form a geometrical progression. The time will be increased by a fraction of the time equal to $\frac{1}{2} x \delta$ nearly, and the common ratio by which the arcs decrease is $\varepsilon^{-\frac{\pi}{2} x^{\prime} \delta}$ nearly. The less the sphere the greater are $x$ and $x^{\prime}$; the more, therefore, is the time altered and the quicker does the arc of vibration decrease.

If a sphere move uniformly in a fluid with friction, we 
may determine the resistance opposed to its motion by the fluid. The calculation is not brief, but the result arrived at for the resistance is

$$
\mathrm{R}=6 \pi \mu^{\prime} \rho \alpha \mathrm{V},
$$

where $\mathrm{V}$ is the velocity of the sphere and $\mu^{\prime}$ the constant ratio of $\mu$ to $\rho$.

The calculation is founded on the supposition that $\mathrm{V}$ is so small that its square may be neglected. The part of the resistance, therefore, which depends on the simple power of the velocity, does not vary as the surface exposed to the fluid, but simply as the radius of the sphere. This becomes important when we apply the above formula to determine the terminal velocity of a very small sphere falling in a fluid under the action of gravity.

Let $\sigma$ be the specific gravity of the sphere, $\rho$, as before, that of the fluid; then, if $\mathrm{V}$ be the terminal velocity, we have

$$
\begin{array}{r}
6 \pi \mu^{\prime} \rho a \quad \mathrm{~V}=\frac{4}{3} \pi g(\sigma-\rho) a^{3}, \\
\therefore \mathrm{V}=\frac{2 g}{9 \mu^{\prime}} \cdot\left(\frac{\sigma}{\rho}-1\right) a^{2} .
\end{array}
$$

According to the usual theors, the terminal velocity would have been

$$
\mathrm{V}=4 \sqrt{\frac{g}{3}\left(\frac{\sigma}{\rho}-1\right) a} .
$$

Thus $\mathrm{V}$ varies as $a^{2}$ instead of $\sqrt{a}$, and therefore becomes very much smaller, when $a$ is small, than that given by the usual theory. Professor Stokes calculated that for a sphere one thousandth of an inch in diameter, the terminal velocity is 1.593 inches per second; for a sphere one tenthousandth of an inch in diameter, the velocity is 01593 . Those given by the usual theory are respectively 32.07 and $10 \cdot 14$ inches per second. 
The suspension of clouds may, therefore, be explained according to this theory. The minute drops are really falling with very small velocities.

The investigations for the motion of cylinders have also been effected, but it will detain us too long to consider all the results. One fact in connexion with the cylinder is remarkable. The motion of the fluid in immediate contact of a sphere moving in a fluid is the same as that of the sphere, and as we go from the surface into the depths of the fluid, the velocity differs more and more from that of the sphere, and finally ends in being zero. The sphere by the friction of its surface tends continually to increase the mass of fluid it drags with it; the friction of the fluid at a distance tends continually to diminish it. These two in the case of a sphere tend to equality, and the motion is ultimately uniform. Not so in the case of a cylinder: the increase on the quantity of fluid carried gains on the decrease due to the friction of the fluid, and the quantity carried increases continually. The velocity must therefore decrease continually.

Professor Stokes has also submitted his results to a comparison with experiment. He first proceeds to obtain $\mu^{\prime}$, the only constant at his disposal: the results of Baily with cylindrical rods give

$$
\sqrt{\mu^{\prime}}=\cdot 116 .
$$

"It is to be remembered that $\sqrt{\mu^{\prime}}$ expresses a length divided by the square root of a time, and the numerical value above given is adapted to an English inch as the unit of length, and a second of mean solar time as the unit of time."

Let us take one instance of his series of comparisons at random: let them be the experiments of Baily on spheres attached to fine wires. Allowance is made for the wire by the theory of the motion of a cylinder. Allowance is also made for the confined space, which is estimated as 
being nearly the same as that given by the ordinary $\mathrm{Hy}$ drodynamic theory. Thus in one of Baily's brass $1 \frac{1}{2}$ inch sphere, the several parts of $n$ were, for buoyancy 1 ,-for inertia, on the common theory, 5 -additional for inertia on account of internal friction $\cdot 202$ - correction for wire $\cdot 012$ - correction for confined space $\cdot 032$-total 1·746. Result of experiment, $1 \cdot 755$, - error $\frac{1}{194}$.

(4.) In the fourteenth volume of the Edinburgh Transactions there will be found an interesting account of some experiments by Scott Russell on the resistance experienced by floating bodies in their progress through the water. As the object was to determine the resistance to ships, the experiments were conducted on a large scale, and the bodies used were vessels of 31 to 75 feet long. The velocities varied from 3 to 15 miles an hour. Two points are worthy of notice in these experiments.

1. The Emersion of the solid body from the fluid. The ship does not draw as much water when it is in motion as when it is at rest. This is manifestly caused by the resolved part of the resistance in a vertical direction.

2. The motion of the boat does not excite currents in the water, but generates waves. These waves travel to great distances with a velocity independent of the form of the vessel and, when freely moving, equal to the square root of the product of the depth of the water and gravity.

The position of the boat relative to these waves was remarkable. Calling $h$ the depth of the water, the velocity of a "free" wave will be $\sqrt{g h}$; let $v$ be the velocity of the boat. If $v$ be less than $\sqrt{g} \bar{g}$, the wave will have a tendency to travel a little quicker than the boat, and it was observed that the accumulation of all the waves generated by the boat formed an elevation at the prow and a depression at the stern. Thus the vessel rode on the posterior side of a "forced wave," with its prow elevated above its stern. If $v$ be greater than $\sqrt{g h}$, opposite phe- 
nomena occurred, and the boat rode on the anterior surface of a "forced wave," with its prow depressed below its stern. If the boat were suddenly stopped, the wave became immediately "free," and was propagated forwards with the velocity $\sqrt{g h}$. If the velocity of the boat were equal to that of a free wave, the boat rode on the top, with its prow and stern much more out of water than its middle part.

By making the vessel move with a velocity $=\sqrt{g h}$, the depth of water is increased by the height of the wave, and it is found that by this artifice boats can be carried without grounding over shallow parts of the canal. Professor Airy has offered an explanation of the phenomena, to which we shall allude when we come to discuss waves.

As may be expected, these waves considerably affect the resistance offered to the boat. Accordingly Scott Russell found that the resistance does not follow the ratio of the square of the velocity, except when the velocity is small and the depth of the fluid considerable.

The resistance was found to increase quicker than in the ratio of the square of the velocity, as the velocity approached a certain quantity determined by the depth of the fluid. After this point of maximum, the resistance actually decreases as the velocity increases, until the velocity is equal to the velocity of propagation of a free wave, and the resistance is here less than that due to the square of the velocity. After this the resistance increases with the velocity, but in a ratio slower than that due to the square of the velocity. According to the law of progression established, the resistance would reach a second point of maximum when the velocity shall have attained a certain quantity, greater than any obtained in the experiments.

The best velocity for a boat to travel at in a canal is therefore $\sqrt{g h}$. That the resistance should be less for this velocity than for any other is clear, for then the boat rides on the top of the wave, and the water is moving in the 
same direction as the boat. Thus even on the ordinary theory, the resistance (calling $u$ and $v$ the velocities of the water and boat) would depend on $x(v-u)^{2}$ instead of $x v^{2}$. 


\title{
NOTE VI.
}

\author{
PENDULUM.
}

There are two properties of the cycloid which enable us to determine, with considerable ease, the motion of a

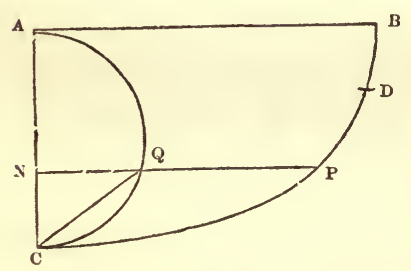
particle oscillating in a resisting medium, and constrained to describe that curve. Let C P B be any cycloid with the tangent at the cusp $\mathrm{B}$ vertical. Let $\mathrm{CA}$ be its axis, and on it describe the semicircle $\mathrm{C} Q \mathrm{~A}$. Now, $\mathrm{P}$ being any point on the cycloid, draw $\mathrm{PQN}$ perpendicular to $\mathrm{CA}$, cutting the circle in $\mathrm{Q}$, and join $\mathrm{CQ}$. Then the properties referred to are,

1. The tangent at $\mathrm{P}$ is parallel to $\mathrm{C} \mathrm{Q}$.

2. The arc $\mathrm{C} \mathrm{P}$ is twice the chord $\mathrm{C} \mathrm{Q}$.

$A$ particle is constrained to move in a cycloid under the action of gravity, to determine the motion.

Let $m$ be the mass of the particle, $w$ its weight, and let $l$ be twice the diameter of the generating circle. Suppose $\mathrm{P}$ to be the position of the particle at any time $t$, and let the arc $\mathrm{C} \mathrm{P}$ be $s$.

The weight of the particle may be resolved into two, one along the normal and one along the tangent. The effect of the former will depend on the manner in which 
the particle is constrained to keep its path. If it be suspended in a peculiar manner by a string, it will merely increase the tension, and thus produce no effect on the motion. The force along the tangent will tend to pull the particle towards C. Since the tangent at $\mathrm{P}$ is parallel to $\mathrm{C} \mathrm{Q}$, this resolved force will clearly be $\omega \cos \mathrm{Q} \mathrm{CA}$; and since the angle $\mathrm{CQA}$ in the semicircle $\mathrm{CQA}$ is a right angle, and the arc C.P is twice the chord $\mathrm{C} Q$, we have the above force, $=\omega \frac{\mathrm{C} \mathrm{Q}}{\mathrm{C} \mathrm{A}}=\omega \frac{s}{l}$.

Hence it appears that the moving force varies as $s$; that is, as the distance from $\mathrm{C}$, measured along the arc. The motion of a particle under a force varying as the distance has already been investigated. The motion in the present case is a particular case of that theorem; viz., that case in which the particle always moves in the direction in which the force acts. It was shown that all particles describe their orbits round a force $=\mu r$ in the same time, viz., $\frac{2 \pi}{\sqrt{\mu}}$. Hence, in our case, the time of oscillation is independent of the length of the arc described, and is equal to $\pi \sqrt{\frac{\overline{l m}}{\omega}}$. Calling this time $\mathrm{T}$, we have,

$$
\mathrm{T}=\pi \sqrt{\frac{l m}{\omega}} .
$$

When the arc of oscillation is circular, the preceding investigation must be somewhat modified. When the arc is considerable, the time of an oscillation cannot be found in finite terms. But in all practical cases the are bears only a small ratio to the radius, and the time of a whole oscillation is then found to be

$$
\mathrm{T}=\pi \sqrt{\frac{\bar{a}}{g}} \cdot\left\{1+\frac{h}{8 a}\right\} \text { nearly, }
$$

B B 3 
where $a$ is the radius and $h$ the vertical height through which the pendulum oscillates. The time is therefore longer than in a cycloid. The time also depends on the length of the arc described. When $a$ is very great and $h$ small, we may often neglect this term and say,

$$
\mathrm{T}=\pi \quad \frac{a}{g} .
$$

We may learn many lessons from this important result.

I. It furnishes us with a method of comparing bodies as to the quantity of matter in each. For we see that for pendulums of the same length

$$
\frac{m}{\omega} \text { varies as } \mathrm{T}^{2} \text {; }
$$

if then we take pendulums of the same weight, we can, by observations on $\tau$, determine the masses or quantities of matter in them. By experiments made with the greatest accuracy, Newton and Bessel always found this ratio constant; so that the weight of a body varies in exact proportion to the quantity of matter in it. This ratio we call $g$. And hence,

$$
\mathrm{T}=\pi \sqrt{\frac{l}{g}} .
$$

By observing the value of $\tau$ for any value of $l$, we can deduce that of $y$, giving, when the unit of time is a second,

$$
g=32 \cdot 18 \text { feet. }
$$

II. The time of oscillation is independent of the arc. By the use, therefore, of a cycloid, we are enabled to prevent any variations of the arc described produced by any irregularities from affecting the rate of going of a clock.

III. Since the time of an oscillation varies as the square 
root of the length of the pendulum, we see that short pendulums must move much quicker than long ones. This is an observation that every one must have made that has entered a clockmaker's shop. But the knowledge of the above ratio enables us to correct the clock when the length of its pendulum has been altered by temperature, or, conversely, to alter the length when we wish to change the rate. Suppose the pendulum to make $n$ oscillations in any given time M. Then clearly,

$$
n=\frac{\mathrm{M}}{\pi} \sqrt{\frac{g}{l}},
$$

taking the logarithmic differential

$$
\frac{\delta n}{n}=-\frac{1}{2} \frac{\delta}{l}
$$

This shows that a change of length equal to a fraction $\alpha$ of the whole length corresponds in any number of oscillations to a loss of a fraction $\frac{\alpha}{2}$ of that number of oscillations.

IV. Another use of the pendulum is to determine the force of gravity and its variations over the surface of the earth. When this has been done we have seen how the true figure of the earth may be deduced. We have now only to describe briefly how this application is made. It will require, of course, great accuracy of observation. The first requisite is to determine in the pendulum experimented on the distance of the centre of oscillation from the axis of suspension. This is the length called $l$ in our formula. There is a variety of practical ways of determining this, which we shall not enter into now. The second requisite is to determine the time of one oscillation of this pendulum, and this is done by noting the time of any large number of observations, and dividing by this number; thus any error made in observing the time of beginning or ending is rendered insensible. The results 
of observation may be tabulated in any form; a good way of exhibiting them is by deducing from them the length of the seconds pendulum. If $\lambda$ be the length, we have,

$$
g=\pi^{2} \lambda
$$

thus $g$ is always proportional to $\lambda$.

But if we wish to ensure accuracy in our results it is clear that we must allow for the effects of all causes that may affect the time of an oscillation besides gravity. These corrections are called "Reductions." Let us briefly consider what they are.

1. If the centre of oscillation does not describe a cycloid, allowance must be made for the alteration of time as depending on the arc described. This is called the "reduction to infinitely small arcs." If the arc of vibration be $n^{\circ}$ on each side of the vertical, then the time of an oscillation will be nearly

$$
\pi \sqrt{\frac{l}{g}}\left(1+\frac{1}{4} \cdot \sin ^{2} \frac{n}{2}\right) ;
$$

hence the time of an oscillation must be divided by this latter factor. If the arcs remained constant this would be sufficient; but it is found that the arcs continually decrease by friction and resistance of air. Experiment shows* that this decrease is in geometrical progression. Taking this for granted, it is easy to deduce that the mean time of one vibration will be

$$
\pi \sqrt{\frac{l}{g}}\left\{1+\frac{n^{2}-n^{2}}{\log n-\log n^{\prime}} \cdot \frac{\sin ^{2} 1^{0}}{32}\right\}
$$

where $n, n^{\prime}$ are the first and last arcs described, and $m$ their number.

The time of an oscillation, as deduced from observation, 
must therefore be divided by the quantity between the brackets.

'This expression has been deduced on the supposition that the point of suspension remains fixed. In the Philosophical Transactions for 1831, Col. Sabine has pointed out that this is not always the case. A further correction in such cases is, therefore, necessary.

.2 The effect of the resistance of the air must be allowed for ; this is called the "Reduction to a vacuum." This will be considered in the propositions that follow relative to the motion of a pendulum in a resisting medium. This correction consists of two parts, that for the buoyancy and that for the inertia of the air. If $m$ be the mass of the pendulum, $m^{\prime}$ that of the fluid displaced. The effect of buoyancy is clearly to decrease the acting force in the ratio $m-m^{\prime}$ to $m$; and the effect of the inertia is to increase the mass moved by $x m^{\prime}$, where $x$ is a quantity to be hereafter determined. The time is therefore increased in the ratio

$$
\sqrt{\frac{m+x m^{\prime}}{m-m^{\prime}}: 1}
$$

Let $z$ be the ratio of the specific gravity of the medium to that of the body, and let $z$ be very small, then the above is equal to

$$
1+\frac{x+1}{2} z
$$

the observed time of an oscillation must be divided therefore by this quantity.

3. The time of an oscillation, thus corrected, enables us to find the length of the seconds pendulum at the place of observation. This gives the force of gravity as affected by the attraction of all the irregularities of the earth's surface near at hand. To render the results obtained in different places comparable with each other, we must reduce 
them all to one given surface. For many years it was the custom to reduce all observations to the level of the sea, taking account only of the height of the station. But in the Phil. Trans. for 1819, Dr. Young pointed out that this correction was too great, as it entirely neglected the attraction of the intervening strata. Supposing the observation to have been made on table land of an altitude $h$ above the level of the sea, the attraction of this stratum will clearly differ but little from that of an infinite plane of the same thickness and density. Let $\sigma$ be the density of the land, then this attraction is well known to be $2 \pi \sigma h$. Let $\rho$ be the mean density of the earth and $a$ its radius, then $g$, the force of gravity, will be $\frac{4}{3} \pi \rho a=g$; hence the correction for the attraction of the intervening land will be $-\frac{3}{2} \cdot \frac{\sigma}{\rho} \cdot \frac{h}{a} g$. But the old correction for distance is clearly $\frac{2 h}{a} g$; hence the total correction will be

$$
\frac{2 h}{a} g\left(1-\frac{3}{4} \frac{\sigma}{\rho}\right) \text {. }
$$

The quantity $\frac{\sigma}{\rho}$ for most rocks on the earth's surface is nearly $\frac{2 \cdot 5}{5 \cdot 5}$; hence the old reduction must be multiplied by 66. This corrected result gives the force of gravity at the level of the sea, if all the land above this level were cut off, and the sea constrained to keep its present level. As the sea would tend in such a case to change its level, it has been pointed out within the last three or four years that a further correction is necessary, if we wished to reduce the result the surface of that spheroid which most nearly represents the earth. But the above is sufficient for tabulating the results.

$\mathrm{V}$. Another important use of the pendulum is to determine the law of the resistance of different fluids to bodies 
moving in them. By experiment we can determine the motion of a pendulum in air or water, and thus we shall be able to test any assumed law of resistance. Newton has devoted nearly the whole of his sixth section to this question. 


\section{NOTE VII.}

MOTION OF FLUIDS RUNNLNG OUT OF SMALL ORIFICES.

(3.) MANY attempts were made by the mathematicians who followed Newton to improve and extend the theory of the motion of water running out of vases. But though the results were often correct, yet the principles on which their solutions were founded did not possess a character sufficiently elementary to entitle them to be called axioms. Maclaurin gave an extension of Newton's theory in his "Traité des Fluxions," liv. i. chap. 12. He argues that the weight of water must be divided into three parts: the first, which accelerates the motion of the surface, and is equal to $\mathrm{A} h \frac{d \mathrm{U}}{d t}$ (following the preceding notation), the second, which presses on the base of the vessel, and the third which in the little time $\delta t$ communicates a velocity $\mathrm{V}-\mathrm{U}$ to the mass V B $\delta t$ of fluid that flows out of the vessel in that time. He assumes that these last two forces are always in a constant ratio, which he supposes to be that of the mass of solidified fluid to the mass of water in Newton's cataract.

Daniel Bernoulli * gave a theory founded on the principle that the fluid may be divided into horizontal strata which remain horizontal throughout the motion and descend with a velocity reciprocally proportional to the area

* Traité d'Hydrodynamique. 
of the section of the vessel that the stratum is passing. $\mathrm{He}$ then applied the principle of the conservation of vis viva to determine the velocity of efflux. Bernoulli was led to this hypothesis by observing the manner in which particles of Spanish wax immersed in the water moved along with the fluid. A theory thus founded on observation is usually a great step in advance. Even now, in certain cases, we are obliged to have recourse to this very assumption. John Bernoulli (Hydraulique) gave a different theory. Taking a stratum of the fluid, he replaced the force which would produce its motion by another supposed to act at the surface of the fluid. Then by integration he obtained the whole force that acting at the surface would produce the whole motion of the fluid, and this force he assumes to be equal to the whole weight of the fluid. There would now be no advantage in dwelling on these or any similar investigations. By the aid of D'Alembert's principle we have now correct equations, giving the motion of fluids under all circumstances. We shall therefore pass on to the more satisfactory solutions that Poisson (Traité de Mecanique, tom. ii. chap. iii.) gave of the question.

(4.) In modern times, it is usual to deduce the velocity of efflux from the equations of motions. The simplest case is when the motion is steady. Let the surface of the fluid be always horizontal, and retained at the same level; let the area be $A$, and suppose all its parts to have the same velocity (U) in a direction vertically downwards. Let $\mathrm{B}$ be the area of the section of the fluid at the vena contracta, $\mathrm{V}$ the velocity of the fluid at that point. A complete solution of the question ought to determine both $\mathrm{B}$ and $\mathrm{V}$ in terms of the diameter of the hole and the other circumstances of the problem. According to the ordinary notation we have,

$$
p=-g y-\frac{v^{2}}{2}+\mathrm{C},
$$


along the surface $y=0, v=\mathrm{U}, p=\pi$, the pressure of the atmosphere;

$$
\therefore \pi=-\frac{\mathrm{U}^{2}}{2}+\mathrm{C} \text {. }
$$

At the vena contracta, the fluid moves with the same velocity throughout the section, and along its surface we have

$$
\pi=-g h-\frac{\mathrm{V}^{2}}{2}+\mathrm{C},
$$

$h$ being its depth below the surface of the fluid; hence we have

$$
\left.\begin{array}{rl}
\mathrm{V}^{2} & =\mathrm{U}^{2}+2 g h . \\
\text { But } \quad \mathrm{BV} & =\mathrm{A} \mathrm{U} .
\end{array}\right\}
$$

These are not sufficient to determine all the unknown quantities. Sufficient equations would have been found if we could have integrated all the equations of motion. The quantity $h$ differs but little from the depth of the orifice below the vena contracta. The quantity B also depends chiefly on the nature of the orifice, and can be measured. The quantity $U$ is unknown, and must be such that the motion is steady; eliminating we get

$$
V=\sqrt{\frac{2 g h}{1-\left(\frac{B}{A}\right)^{2}}} .
$$

If the orifice be very small, we may reject $\left(\frac{B}{A}\right)$, and we get

$$
\mathrm{V}=\sqrt{2 g h} .
$$

When the velocity of the surface is not such as to render the motion steady, the equations cannot be integrated, except on the supposition that the orifice is excessively small. It that case we may assume with sufficient accuracy, that the particles of fluid once in a horizontal 
section, always remain in one. This is called the hypothesis of parallel sections. Supposing the fluid to begin to move from rest, it can be shown that the motion, whatever be the form of the vessel, soon becomes steady. So that we may in general use the preceding formula.

If the vessel be allowed to empty itself, the solution can still be found on the same supposition. If $z$ be the height of the surface of the water in a cylindrical vessel above the vena contracta, and $h$ be the initial value of $z$ when the motion is supposed to begin from rest,

$$
\mathrm{V}^{2}=\frac{2 g z}{1-\left.2 \frac{\mathrm{B}}{\mathrm{A}}\right|^{2}}-\left.\left.\frac{2 g h}{1-2 \overline{\mathrm{B}}{ }^{2}} \cdot \bar{z}\right|^{\overline{\mathrm{A}}}\right|^{2}-1 .
$$

If $B$ be very small, this expression becomes very nearly

$$
\mathrm{V}^{2}=2 g z \text {. }
$$

If some time has elapsed, so that $\frac{z}{h}$ is a small fraction, we have

$$
\mathrm{V}^{2}=\frac{2 g z}{1-2\left(\frac{\mathrm{B}}{\mathrm{A}}\right)^{2}} .
$$

The velocity being then very small, the quantity of liquid that has run out of the ressel in the time $t$ will be very nearly

$$
q=\mathrm{B} t \sqrt{2 g h} ;
$$

if the orifice be an aperture in a very thin plate, the value of $\mathrm{B}$, the area of the vena contracta is about $\frac{62}{100}$ of the area of the orifice. Calling this area $\alpha$, the expenditure will be nearly*

* Poisson, Hydrodynamique, vol. ii. 


$$
q=\cdot 62 \cdot \alpha \cdot t \cdot \sqrt{2 g h} .
$$

But the efflux is very much influenced by the nature of the orifice. If a small vertical cylinder, or adjutage, be applied to the exterior of the orifice, the discharge is greatly increased, and will be nearly

$$
q=8 \propto t \sqrt{2 g h} ;
$$

if, on the contrary, the cylinder be placed on the interior of the orifice, the discharge is decreased, and will be nearly

$$
q=\cdot 5 a t \sqrt{2 g h} .
$$

Venturi found an adjutage, consisting of two conical portions having their smaller ends united at the commencement of the vena contracta, to give a very large discharge.

(5.) An attempt has also been made to determine the velocity of efflux of an elastic fluid rushing out of a small orifice. The problem is a very difficult one, and no satisfactory solution has been found.

Let a quantity of any gas be compressed at density D and pressure $\mathrm{P}$, in any vessel $\mathrm{A}$; let it be allowed to run out by a small orifice into a vessel $A^{\prime}$, filled with the same kind of gas at density $\mathrm{D}^{\prime}$ and pressure $\mathrm{P}^{\prime}$. It is required to determine the rate at which the gas runs from the first vessel into the second.

Draw a line of motion from the interior of the vessel A to the interior of $A^{\prime}$, so that at any instant all the particles in this curve are moving along tangents to the curve. The motion of the particles at the two extremities of this line will be infinitesimal when the aperture is very small compared with the size of the vessels. Since the aperture is very small, the motion may be considered for any short time steady; hence the equation 


$$
\int \frac{d p}{\rho}=\mathrm{C}-\frac{v^{2}}{2}
$$

will be approximately true. The quantities $p, \rho, v$, in this equation express the pressure, density, and velocity at any point of the curve. Integrating from the extremity of the curre in the ressel $A$ to the orifice, we have

$$
\int_{\mathrm{P}}^{p} \frac{d p}{\rho}=-\frac{v^{2}}{2} \text {. }
$$

Supposing the difference between $\mathrm{P}, \mathrm{P}^{\prime}$ not too great, the fluid will rush into the receiver in a stream, and there will be a backward current on each side to diffuse the gas over the whole vessel. Taking some section where the fluid may be supposed to move with the same velocity throughout, the quantity that runs past in a unit of time, measured in volumes of gas at density $D$, is

$$
\mathrm{V}=m \frac{\rho}{\mathrm{D}} \sqrt{2 \int_{p}^{\mathrm{P}} \frac{d p}{\rho}} ; \quad-\quad-
$$

where $p$ and $\rho$ express the pressure and density at the section, and $m$ is a numerical coefficient depending on the nature of the orifice and the unit in which $p$ is measured. We are ignorant of the true values of $p$ and $p$; but if the vessel be very large, and the difference of pressures $P, P^{\prime}$ small, the gas on each side of the stream will be nearly stagnant, and we may substitute for $p, \rho$, the values of $\mathrm{P}^{\prime}, \mathrm{D}^{\prime}$, the mean pressure and density in the receiver. If $\mathrm{P}-\mathrm{P}^{\prime}$ be so small that we can neglect its square, this formula will become

$$
\mathrm{V}=m \sqrt{2 \frac{\mathrm{P}}{\mathrm{D}}\left(1-\frac{\mathrm{P}^{\prime}}{\mathrm{P}}\right)} ; \quad-\quad-\quad \text { (2.) }
$$

an expression which is in tolerable accordance with the results of experiment. But if $\mathrm{P}$ and $\mathrm{P}^{\prime}$ are not nearly equal, this is no longer even an approximation to the truth. 
Suppose we reject the effect of changes of temperature, we have, by Boyle's law, $p=x \rho$, whence the expression (1.) easily gives

$$
\mathrm{V}=m \frac{\mathrm{P}^{\prime}}{\mathrm{P}} \sqrt{2 \frac{\mathrm{P}}{\log _{\varepsilon} \frac{\mathrm{P}}{\mathrm{P}^{\prime}}}} \quad-
$$

MM. Barré de Saint Venant and Wantzel * have undertaken the task of testing these expressions by actual experiment. They have pointed out that the formula (3.) could not possibly be accurate, for it gives the velocity of efflux a maximum when $\frac{\mathrm{P}^{\prime}}{\mathrm{P}}=\cdot 60653$, so that the velocity would actually be less the smaller the quantity of air in the vessel $\mathrm{A}^{\prime}$, provided it be less than a certain quantity. The velocity would vanish when $\mathrm{P}^{\prime}=0$, which leads to the extraordinary result that a gas cannot rush into a vacuum. If in order not to neglect the changes of temperature, we put $p=x \rho^{2}$, we get the expression

$$
\mathrm{V}=m\left(\frac{p}{\mathrm{P}}\right)^{\frac{1}{\gamma}} \cdot \sqrt{2 \frac{\mathrm{P}}{\mathrm{D}} \cdot \frac{\left(1-\frac{p}{\mathrm{P}}\right)^{1-\frac{1}{\gamma}}}{1-\frac{1}{\gamma}}} .
$$

If we knew the values of $p$ and $\gamma$ this would no doubt give accurate results. But when we substitute $p=\mathrm{P}^{\prime}$, we are led to results as absurd as those we have just mentioned. The truth is, that, other things being the same, the velocity of efflux of air by an orifice is always greater the more the pressure in one vessel exceeds that in the other. So long as $\frac{\mathrm{P}^{\prime}}{\mathrm{P}}$ is not greater than $\cdot 3$ or $\cdot 4$, the efflux or the quantity of air that has flowed out in a unit of time is sensibly the same; that efflux diminishes slowly at first, more quickly afterwards, as the pressures approach equality, and it becomes nothing after a finite time, when

* Comptes Rendus, vol. ix. and xvii. 
the pressures become the same in the two vessels which the orifice puts in'communication. These are the results of MM. Venant and Wantzel; they have even deduced an empirical law to represent the velocity. The efflux per metre of orifice in volume at pressure $\mathrm{P}$ will be

$$
\mathrm{V}=\frac{m \cdot \sqrt{2 \frac{\mathrm{P}}{\mathrm{D}}} \sqrt{1-\frac{\mathrm{P}^{\prime}}{\mathrm{P}}}}{1+\frac{1}{n}\left(1-\frac{\mathrm{P}^{\prime}}{\mathrm{P}}\right)^{\frac{n^{\prime}+1}{2}}}
$$

where $m, n$ are two numbers which depend on the nature of the orifice, and $n^{\prime}$ a number to which we give a value a little greater than $n$, that the formula may give a nearly constant value to $\mathrm{V}$ between the limits already stated. 


\section{NOTE VIII.}

MOTION OF WAVES ON THE SURFACE OF WATER.

IT appears that it is not every form of waves which can be freely propagated with a uniform velocity without any change of form. The only case in which this is possible, is when the vertical section by a plane passing through the direction of propagation is, to a first approximation, the curve of sines. A solitary wave* cannot, therefore, be propagated without change of form; a result confirmed by Mr. Russell's experiments. If the form of the waves were accurately the curve of sines, they would be exactly similar above and below the undisturbed level. This common experience shows not to be the case. On carrying our investigation to a second approximation, on the supposition that the depth of the water is much greater than the length of the wave, it is found that the altitude of the top above the undisturbed level is greater than the depth of the hollow beneath it. The top of the waves are more pointed than the hollows. Such a wave is propagated without any change in its form. If the depth were much less than the length of the wave, the wave will undergo certain changes, which we shall have occasion to refer to presently.

The motion of the water during the uniform propagation of the wave is greatest at the surface, and decreases rapidly with the depth; but the rate of decrease varies very nearly inversely as the length of the wave. Thus if the depth of the water be much less than the length of a wave,

* Camb. Phil. Trans. vol. viii. 
the motion is nearly the same at all depths; if much greater the water near the bottom is almost at rest. Each particle of water describes an ellipse. Those at the bottom oscillate in straight lines, and in very deep water those at the surface describe circles.

When the channel is not uniform the question of the motion is much more difficult. Professor Kelland has investigated one case of this kind, for which we refer our readers to the Edin. Trans. for 1841.

The motion of any series of oscillatory waves will, of course, tend to subside from the effects of internal friction. The rate at which this takes place depends very much on the length of the wave. The magnitude of the wave is found to depend on the factor *

$$
c=c_{0} \cdot \varepsilon^{-\frac{16 \pi^{2} \mu^{\prime} t}{\lambda^{2}}}
$$

where $\lambda$ is the length of the wave, $\mu^{\prime}$ a numerical quantity depending on the amount of internal friction in the fluid, $c_{0}, c$ the values of the factor at the times zero and $t$. The value of $\sqrt{\mu^{\prime}}$ for water is 0564 , an inch and a second being the units of space and time. "Suppose, first, that $\lambda$ is two inches and $t$ ten seconds, then $16 \pi^{2} \mu^{\prime} t \lambda^{-2}=$ $1 \cdot 256$ and $c: c_{0}:: 1: 0 \cdot 2848$, so that the height of the waves which varies as $c$ is only about a quarter of what it was; accordingly the ripples excited on a small pool by a puff of wind rapidly subside when the exciting cause ceases to act. Now suppose that $\lambda$ is 40 fathoms, or 2880 inches, and that $t$ is 86,400 seconds, or a whole day. In this case $16 \pi^{2} \mu^{\prime} t \lambda^{-2}$ is equal to only 0.005232 , so that by the end of an entire day, in which time waves of this length would travel 574 English miles, the height would be diminished by little more than one two-hundredth part in consequence of friction. Accordingly the long swells of the ocean are but little allayed by friction, and 
at last break on some shore situated at the distance of perhaps hundreds of miles from the region where they were first excited."

When the wave is very long it is easy to give a short demonstration of its most useful properties. And this is the more advantageous, for, as we shall presently see, the tide is nothing more than an exceedingly long wave.

Suppose we have a rectilinear channel of uniform section; let $y$ be the elevation of the water above the mean level at a time $t$, and at a distance $x$ measured along the channel from the origin of measurement. Let $k$ be the greatest value of $y$. Let $h$ be the depth of the channel, $\lambda$ the length of the wave travelling along the canal. Then by hypothesis $\lambda$ is very great. Suppose the particle of water which when undisturbed to have been at a distance $x$ from the origin, to be at the distance $x+\mathrm{X}$ at the time $t$. First let there be two imaginary planes placed at the beginning and ending of the long wave. The mass* of water elevated above the mean level will be comparable with $\lambda k$, while the water which has passed the two imaginary planes will be comparable with $h \mathrm{X}$. These two quantities, then, must be of the same order; hence

$$
k \text { is of the order } \frac{h \mathrm{X}}{\lambda} \text {; }
$$

and, as $\lambda$ is very great, this must be very much smaller than $\mathrm{X}$. Now $\mathrm{X}$ is itself supposed a small quantity; hence $k$ may be altogether rejected when compared with $\mathbf{X}$, and we must suppose the elevations performed by the horizontal motions of the fluid pressing together some parts, and thus raising it above the general level. So far as the vertical motion is concerned, we may consider the fluid in equilibrium, and hence may use the ordinary equations of hydrostatics to find the pressure at any depth.

We shall now show that if we assume the displacement

* Camb. Dub. Mathematical Journal, Nov. 1849. 
$\mathrm{X}$ of the particle which was situated at the distance $x$ from the origin to be represented by

$$
\mathrm{X}=a \sin (n t-m x),
$$

that then all the conditions required for a possible motion will be satisfied. If this expression agree with the initial conditions and all the other circumstances of the motions, it will represent the actual motion. The whole of the reasoning is, step for step, the same as that for the motion of sound in a tube.

Since this form of $\mathbf{X}$ is independent of the depth, the water will be agitated to the bottom, and the particles once in a vertical plane will always remain in one. If then two planes be taken at a distance $d x$ when the fluid is at rest, the mass of water between them will always remain the same. But this mass before the motion was $h d x$, and at the time $t$ it is

$$
\begin{aligned}
& (h+y)(d x+d \mathrm{X})=h d x, \\
& \therefore y=-h \frac{d \mathrm{X}}{d x}-y \frac{d \mathrm{X}}{d x} ;
\end{aligned}
$$

the last term is very small, for both $y$ and $\mathrm{X}$ are small, and therefore may be rejected; hence

$$
\begin{aligned}
y & =-h \frac{d \mathrm{X}}{d x} \\
& =a m h \cdot \cos (n t-m x) .
\end{aligned}
$$

If we consider any element $d x$ of the fluid, the forces that make it move are the pressures on its two ends. These are, by hydrostatics,

$$
g(y+z) \text { and } g(y+d y+z),
$$

where $z$ is the depth of the element beneath the mean surface. Hence the moving force is

$$
\begin{aligned}
& g d y, \\
& \text { c c } 4
\end{aligned}
$$


and the mass moved is $d x$; hence the accelerating force is

$$
\begin{gathered}
g \frac{d y}{d x} \\
\text { or } \quad-a m^{2} h g \sin (n t-m x) \\
\text { or } \quad-m^{2} h g \mathrm{X} ;
\end{gathered}
$$

hence the force varies as the displacement. This is well known to lead to the very law assumed for $\mathbf{X}$, provided

$$
\sqrt{m^{2} h g}=n ;
$$

hence the velocity of the wave, which we know is $\frac{n}{m}$, must be $\sqrt{g h}$.

The general equation for the motion of any long wave may be obtained by a generalisation similar to that employed in the proportion corresponding to this in sound. The resulting equations are even the same: we arrive at

$$
\begin{aligned}
\eta & =-h \frac{d \mathrm{X}}{d x} \\
\frac{d^{2} \mathrm{X}}{d t^{2}} & =g h \cdot \frac{d^{2} \mathrm{X}}{d x^{2}},
\end{aligned}
$$

and whatever be the form of the wave, provided only it be very long, and the height of the wave be small compared with the depth of the water, the velocity of transmission will be always the square root of the product of the depth and gravity.

If each particle of the fluid be under the action of forces, the motion may still retain the characteristics of a wave. Such a wave, however, is called a "forced wave," and there is no necessary relation between the velocity of such a wave and the depth of the water. As an example, let each particle be acted on by a horizontal force

$$
\mathrm{F}=f \cdot \sin .(i t-m x) \text {. }
$$


Let us assume the displacement

$$
\begin{gathered}
\mathrm{X}=a \sin .(i t-m x) \\
\therefore \mathrm{F}=\frac{f}{a} \mathrm{X} .
\end{gathered}
$$

But as we have already seen, the water seeking to recover its level causes a horizontal force equal to

$$
-m^{2} h g \mathrm{X} \text {; }
$$

hence the whole force is

$$
-\left(m^{2} h g-\frac{f}{a}\right) \mathrm{X} .
$$

By the same reasoning as before, this coefficient must be equal to $i^{2}$, or

$$
\begin{gathered}
m^{2} h g-\frac{f}{a}=i^{2} ; \\
\text { hence } a=-\frac{f}{i^{2}-m^{2} h g},
\end{gathered}
$$

or the motion in the horizontal direction will be given by

$$
\mathrm{X}=-\frac{f}{i^{2}-m^{2} h g} \cdot \sin (i t-m x),
$$

and the elevation of the water by

$$
y=-\frac{f m h}{i^{2}-m^{2} h g} \cos (i t-m x) .
$$

This proposition will be required when we come to consider the theory of tides. It will also serve to explain in some degree the motion of the waves generated by a boat moving along the surface of a canal. It is not a complete explanation, for the waves observed by Scott Russell were solitary waves; whereas we have supposed in the above reasoning that there is an infinite succession of waves 
following each other. However, we may suppose the grand features of the motion to be similar in the two cases. The above expressions show that the sign of $\mathrm{F}$ will be the same as or different from that of $\frac{d y}{d t}$ according as $\frac{i}{m}$, the velocity of the wave, is greater or less than $\sqrt{g h}$. Taking the former supposition, $\mathrm{F}$ must be positive, or act in the direction of propagation where the water is rising, that is, along the anterior surface of the elevation of the wave, and must act in the opposite direction where the water is falling, that is, along the posterior surface of the elevation. If, on the contrary, the velocity of the wave is less than $\sqrt{g h}$, then $\mathrm{F}$ must act in the direction of propagation along the posterior slope, and in the opposite direction along the anterior slope. A boat can only push the water forwards; hence it must be on that side of the slope on which a forward force is required; that is, it will travel behind or before the wave, according as its velocity is less or greater than $\sqrt{g h}$. There will be no force acting on the other slope of the wave; but the wave will undergo a trifling change of form, and thus similar effects will be produced whether this force does or does not act.

If the velocity of the boat is $\sqrt{g h}$, no force is necessary. If the boat ride on the crest of the wave, it acts on both slopes. If these actions were equal, the particles would have their changes of motion accelerated in front and retarded in rear, and thus the whole change would be the same as if the forces did not act. But the head of the boat, Airy observes, presses the water more than the stern; hence, on the whole, we have a small accelerating force acting on the anterior slope. Hence, that the boat may ride evenly on the crest of the wave, the velocity must be a little greater than $\sqrt{g h}$. This is true in practice.

Many mathematicians have investigated the effects of gradual changes in the depth and breadth of the canal on a 
series of long waves propagated along it. If $h$ and $b$ be the variable depth and breadth, the result arrived at is that the altitude of the wave varies *

$$
\text { inversely as } \sqrt[4]{h} \cdot \sqrt{b} \text {. }
$$

Professor Airy has demonstrated that waves cannot be propagated, strictly speaking, along a canal of variable depth. He shows that if it were possible, we would be led to an equation that cannot be satisfied. Hence, "one at least of the two conditions on which the equations of Hydrodynamics are founded must fail. These are the continuity of the fluid and the equality of pressure in all directions. While the continuity holds, the equal pressure must exist, from the nature of the fluid. Therefore the continuity must cease, or the water become broken. This appears to be the explanation of the broken water which is usually seen on the edge of a shoal, or a ledge of broken rocks, although the whole is covered, perhaps deeply, with water." The Astronomer Royal mentions the instance of Newfoundland, where, when the waves are high, there is a surf caused by the breaking of the waves over the edge of a shoal 500 feet deep. A similar breaking is observed about the line of "no soundings" (that is, where the water suddenly becomes deeper than 600 feet, which at some distance borders the British isles (Encyc. Met., art. Tides and Waves). The wave, on reaching a part of its course where the depth changes suddenly, breaks, because the velocity of the top part forward and of the hollow backwards, is too great to travel with the relocity proper to the new depth. The top part therefore, tumbles forwards into the hollow. The degree of breaking is less and less the longer the wave and the less the change of depth. If the depth alter very slowly, the wave may be considered as only slightly breaking, and is propagated as a changing wave.

* Green on the Motion of Wares, Trans. Camb. Phil. vol. vi. 
Professor Airy has also investigated the manner in which the wind acts to raise the waves. His theoretical conclusions are as follows:- " It is to be understood that either from preceding disturbances, or from the trifling inequalities of the wind while the water is smooth, there are very shallow undulations upon the water. When the wind begins to act, it will at first only increase the height of the waves in every part, and during this time the heads of the waves will be broken. But after a time the waves beginning with the windward shore will be (for a short distance at least) so much increased, that the power of the wind will merely maintain them in that state without any increase; but for all the sea in advance, the wind will still be raising the waves. But as the waves successively obtain that height which corresponding, according to our result, with the height which the wind can just maintain, these waves will no longer be increased, but the waves in advance will still be increased. Thus a wind of given intensity, however long it blows, can only raise the waves at a given point to a certain height; which height, however, will depend upon the distance of that point from the windward shore." 


\section{NOTE IX.}

THE THEORY OF THE TIDES.

II. Since Newton's time many philosophers have turned their attention to the theory of the tides. Newton left it in so imperfect a state that much remained to be done. We shall therefore in the briefest possible manner mention a few of those steps that have been since made. It would require a treatise by itself to discuss them completely. A traveller about to visit a new country looks at the map first: we intend to furnish this map, and must refer to other works those who may desire to study so interesting a subject.

There are at present three theories of the tides. They are usually called, the equilibrium theory, the theory of Laplace, and the ware theory. The differences of these theories will be best understood by considering them separately.

(1.) In the equilibrium theory, the rotation of the earth is supposed to have no effect on the form of the tide, so that the times and heights of high water, and all the other circumstances of the tides, at any place may be calculated on the supposition that both the earth and the moon remain fixed in the position they are in at the moment under consideration. Whether this assumption be legitimate, or not, is a question we shall presently have to consider. The whole equilibrium theory is built on the assumption of its truth.

The general explanation of the tides, according to this theory, is very simple. The moon attracts both the earth 
and the water on the earth. But she attracts the water immediately under her with greater force than she attracts the centre of the earth, and she attracts the centre of the earth with greater force than the water on the opposite side of the earth. But the tides are formed by the position the water assumes relative to the earth. We must therefore consider this as reduced to rest by a force supposed to be applied to every particle of the earth and water equal and opposite to the force with which the moon attracts the centre of the earth. The particles of water immediately under the moon will therefore be drawn towards the moon; those immediately opposite will appear to be drawn from the moon. Thus the water will rise and form a high tide both on that side next the moon and also on the other. Let us now suppose the whole earth covered with water. Each particle of the fluid is under the action of, first, the attraction of the earth, and, secondly, the attraction of the stratum of liquid surrounding the earth: this will depend on the form its surface will assume under, the action of all the forces; thirdly, the disturbing force of the luminary. The form of the water must be determined by means of the equations of fluid equilibrium. The result obtained is that the form will be very nearly a spheroid whose longer axis points to the luminary. If $\theta$ be the zenith distance of the moon at any place and any instant, $c$ her distance, $a$ the mean radius of the earth, $a^{\prime}$ the radius of the solid nucleus of the earth, $\mathrm{E}$ and $\mathrm{M}$ the masses of the earth and moon, $\rho, \rho^{\prime}$ the mean density of the earth and the density of the sea; then the height of the tide at the given place and at the given time will be

$$
\frac{\frac{5}{4} \overline{\mathrm{E}} \cdot\left(\frac{a}{c}\right)^{3} a}{1+\frac{2 \pi a^{\prime 3}}{\mathrm{E}} \overline{\rho-\rho^{\prime}}} \cdot\left(3 \cos ^{2} \theta-1\right) .
$$

The greater the depth of the ocean, or the less $\alpha^{\prime}$ is, the greater is the height of the tide. 
The values of these several ratios are as follows:

$$
\frac{\mathrm{M}}{\mathrm{E}}=\frac{1}{74}, \frac{a}{c}=\frac{1}{60}, \mathrm{E}=\frac{4 \pi}{3} \rho a^{\prime 3},
$$

whence the coefficient is nearly

$$
\frac{1 \cdot 1}{\frac{5}{3}-\frac{\rho^{\prime}}{\rho}} \text { feet. }
$$

By observation $\rho^{\prime}=\frac{1}{5} \rho$ nearly, and in this case the height of the tide is small. But if $\rho^{\prime}$ be much greater, this expression soon becomes considerable, and if the density of the sea had been much greater than that of the earth, the tides would have been very high.

We see also that the height of the tides caused by different luminaries varies as their masses, and inversely as the cubes of their distances.

The coefficient being called $h$, the greatest elevation of the tide will manifestly be $2 h$, and the greatest depression below the spherical surface will be $h$. The elevation is therefore double the depression. But it must be remembered that this spherical surface is not the mean surface of the ocean at the place in question, but the surface as it would be if undisturbed by any luminary.

There will be a similar expression for the tide caused by the sun, and the two together may be expressed by the formula

$$
\begin{aligned}
& \left(1-3 \sin ^{2} \lambda\right)\left\{\mathrm{A} \cdot\left(\frac{c}{c_{0}}\right)^{3}\left(\frac{3}{2} \cos ^{2} \delta-1\right)+\mathrm{A}^{\prime}\left(\frac{c^{\prime}}{c_{0}^{\prime}}\right)^{3}\left(\frac{3}{2} \cos ^{2} \delta^{\prime}-1\right)\right\} \\
& +\frac{3}{2} \sin 2 \lambda\left\{\mathrm{A}\left(\frac{c}{c_{0}}\right)^{3} \sin 2 \delta \cdot \cos (l-\gamma)+\mathrm{A}^{\prime}\left(\frac{c^{\prime}}{c_{0}^{\prime}}\right)^{3} \sin 2 \delta^{\prime} \cos \overline{l-\gamma^{\prime}}\right\} \\
& +\frac{3}{2} \cos ^{2} \lambda\left\{\mathrm{A}\left(\frac{c}{c_{0}}\right)^{3} \cdot \cos ^{2} \delta \cos 2(l-\gamma)+\mathrm{A}^{\prime}\left(\frac{c^{\prime}}{c_{0}^{\prime}}\right)^{3} \cos ^{2} \delta^{\prime} \cos 2 \overline{l-\gamma^{\prime}}\right\}
\end{aligned}
$$

where $l, \lambda$, are the longitude and latitude of the place, $\gamma, \delta$, the right ascensions and declinations of the moon, $c_{0}, c$, her 
mean and actual distances, $\mathrm{A}$ the coefficient of $3 \cos ^{2} \theta-1$ in the preceding formula when the moon is at her mean distance. Accented letters apply to the sun in the same way as unaccented do to the moon.

In this expression we see three kinds of terms. First, those of very long period, and which are not dependent on the hour of the day. These arise from the first line in the above series, and cause differences in the mean elevation of the water, depending on the declination and distances of the disturbing bodies. As the expression contains the factor $3 \sin ^{2} \lambda-1$ there will be no such terms or inequalities for any place whose latitude is $\sin ^{-1} \sqrt{ } \frac{1}{5}$.

Secondly. The terms in the second line going through their values in about a day, they form therefore a diurnal tide. This has no existence for any place on the equator or at the pole, and is greatest in latitude $45^{\circ}$. There will be variations in the magnitude of this tide, depending on the changes of declination and distance of the heavenly bodies.

Thirdly. The terms in the third line go through their values twice in about a day; they form together, therefore, a semi-diurnal tide. This has no existence at the poles, and is greater the nearer the place is to the equator.

The value of this theory may be best stated nearly in the words of the Astronomer Royal:- "The most conspicuous tide on the coasts of Europe at least is the semidiurnal. The acceleration or retard of this tide on the moon's transit does not at one port in a hundred agree in any measure with the result of this theory." "The absolute elevation of the tide is great at one port and small at another, without any relation to the quantity calculated from theory. The proportion of the elevations, however, at the same port in different stages of the lunation agree pretty well with the theory (though not equally at all ports), yet the critical phenomena (spring and neap tides) occur later than the theory gives them." "The peculiar phenomena of river tides are not touched on by this theory." "The diurnal tide ought in these latitudes to be equal, 
or nearly equal, to the semi-diurnal tide. Yet in the Thames it is absolutely insensible, and in other ports, as well of England as of other ports of Europe and America, though discoverable, it is not notorious, and has only been found from the observations made by men of science. It has been found to be very conspicuous at some places near the equator, and some places near the pole, where it ought not to be discoverable, or scarcely discoverable. The tides of longer period have scarcely heen observed."

"Altogether this is one of the most contemptible theories that was ever applied to explain a collection of important physical facts. It is entirely false in its principles, and entirely inapplicable in its results. Yet, strange as it may appear, this theory has been of great use. It has served to show that there are forces in nature following laws which bear a not very distant relation to some of the most conspicuous phenomena of the tides; and what is more important, it has given an algebraic form to its own results, divided into separate parts analogous to the parts into which the tidal phenomena may be divided, admitting easily of calculation and of alteration, and thus at once suggesting the mode of separating the tidal movements, and affording numerical results of theory with which they are to be compared. The greatest mathematicians and the most laborious observers of the present age have agreed equally in rejecting the foundation of this theory, and comparing all their observations with its results. And till theories are perfect (a thing scarcely to be hoped for in any subject, and less in the tides than any other), this is one of the most important uses of theory."

(2.) The next theory to be considered is that of Laplace. In this the phenomena of the tides are treated as a question of hydrodynamics. It is manifest that no true theory can proceed on any other supposition. We may, as usual, suppose the centre of the earth reduced to rest by the application to every particle of the earth and sea a sufficient force. This we can do, for we are only concerned with the motion of the sea relatively to the earth. The 
two luminaries must then be supposed to travel round the earth in respectively a month and a year. Then the problem is to determine what motion will take place in any wide expanse of ocean when acted on by these forces, the earth and sea being supposed to have a rotation round a fixed axis.

But such is the difficulty of the investigation, that if we wish to get any result at all, we must make some suppositions that are not quite applicable to the earth. We must suppose the earth to be entirely covered by water, whose depth is the same along any parallel of latitude, which may be expressed by the formula

$$
\gamma=l\left(1-q \cos ^{2} \theta\right)
$$

where $\theta$ is the polar distance of the parallel of latitude, $l$ the depth at the equator, $q$ a constant depending on the rate at which the depth decreases as we approach the pole. The investigations of Laplace are very difficult: the same results have been obtained by a different but simpler process by Professor Airy in his article on Tides and Waves in the Encyclopædia Metropolitana.

The result of Laplace * is that there are " trois espèces d'oscillations." As these unite without confounding themselves, they can be considered separately.

Des oscillations de la première espèce.-These are tides of long period, and depend on the positions of the luminaries, the depth of the sea as compared with the radius of the earth, the time of rotation of the earth, the force of gravity at its surface, and the latitude of the place, but not on the hour of the day. Formulæ are found to express their magnitude, and these, when no allowances are made for friction and other causes of resistance, are not the same as those given by the equilibrium theory. Laplace, however, supposing the resistance to vary as the velocity, and the tide to be exceedingly small (an assumption founded on observation), shows that we may calculate these oscilla-

* Mec. Celeste, vol. ii. p. 313. 
tions as if the fluid at each moment put itself in equilibrium under the action of the luminary which attracts it. And the error is less, the slower the motion of the luminary; it is therefore insensible for the sun, and we may even assume it true for the moon. This is calculated on the supposition that the resistance varies as the velocity, but it will be, clearly, also true whatever be the law of the resistance.

Des oscillations de la seconde espèce. - These are oscillations which go through their period in about a day, and constitute therefore properly a diurnal tide. It is found that this tide does not exist either at the equator or the poles, and it is greatest about latitude $45^{\circ}$. It will disappear in every latitude if the depth of the sea be uniform, - a result as remarkable as it was unexpected. But this only refers to the elevation of the water. The diurnal variations of the horizontal motions caused by these terms would still remain. The expression for the elevation of the water is found to change sign with $q$; so that if the water be shallower at the poles than at the equator, and its depth be less than seven miles, low water occurs at the transit of the luminary, if deeper at the poles, high water; provided the transit of the luminary takes place on the same side of the equator as the place of observation; and the reverse occurs if the transit takes place on the opposite side.

Des oscillations de la troisième espèce.-These oscillations go through their period in about half a day. They constitute a semi-diurnal tide, having two high waters and two low waters every day. If the depth vary as the square of the cosine of the latitude, the expression for the elevation of the tide shows that there will be low or high water at any place at the moment when the luminary crosses the meridian, according as the depth of the sea is less or greater than about seven miles. Laplace has also investigated the nature of these tides in a sea of uniform depth, but the Astronomer Royal has pointed out an error in his process, D $\mathrm{D} 2$ 
the effect of which is to vitiate his results, and, therefore, also, all the numerical calculations founded on them.

This theory is certainly a wonderful step in advance. The only defects are those that the imperfection of our mathematical knowledge renders imperative. The results show how false that assumption is on which the equilibrium theory is built. That theory could never lead to the result that there must be, under certain circumstances, low water directly under the luminary. The theory of Laplace is, indeed, founded on suppositions as to the depth of the sea; but still we may expect these results to bear some analogy to the phenomena we actually meet with. Further, only the principal terms are considered, and thus the effects on the tides caused by the difference of the motions of the sun and moon are not calculated.

(3.) The third theory may be called the wave theory. It is most successful exactly where all the other theories become so inapplicable as evidently to be quite useless. Its peculiar merit is the discussion of river tides and the explanation of the various phenomena produced by barriers, changes of depth and breadth in the various channels through which the tide has to pass. It is not altogether inadequate to the discussion of certain cases of ocean tides. The problems considered are of two kinds. First, the tides formed in very long canals, the waters of which are acted on by the forces of the sun and moon. These results will be afterwards found to bear some analogy to the case of certain oceans as they exist in nature, bounded by their continental shores. Secondly, the tides formed in rivers which are so short that the action of the disturbing bodies on their waters may be neglected, and the tide may be considered to be freely propagated up the river from the disturbance caused at its mouth by a tidal sea.

This theory is not to be considered as contradictory of that of Laplace. Two truths cannot be opposed to each other. On the contrary, Laplace's equations are required in some of the investigations of this theory. The dif- 
ference is simply this. Laplace's theory aims at determining the tides as formed in extensive sheets of water, and therefore a solution of the Hydrodynamic Equations is taken that is adapted to such a case. The wave theory aims at discussing the tides as formed in long canals, and therefore a solution of the equations is taken as suited to such a case. It is to the labours of Professor Airy that much of the progress that has been made in this subject is due. I may refer to his work in the Encyc. Met. for the demonstration of most of the theorems alluded to in the following sketch.

Let us begin by considering Newton's case of the motion of water in an equatorial channel acted on by the sun, supposed to revolve in the equator. That part of the disturbing force which acts perpendicular to the water, will produce but little effect compared with the tangential force, the weight of a column of fluid equal to the depth acted on by the central force being infinitely less than that of so long a column as a quarter the circumference of the globe acted on by the tangential force. We shall therefore neglect the central force. On applying Laplace's equations it can be proved that the motion in a canal will be the same as if the earth were reduced to rest, and an equal but opposite angular velocity impressed on the disturbing body. By Lunar Theory, the tangential force is known to be

$$
\mathrm{X}=-\frac{3 \mu b}{2 \mathrm{D}^{3}} \cdot \sin 2(p t-\phi),
$$

where $b$ is the radius of the earth, $\mu$ the sun's mass, D its distance from the earth, $p$ the relative angular velocity of the sun and earth, and $\phi$ any angle determining the position of the particle. Then, by the proposition demonstrated in the chapter on waves, the altitude will be

$$
\mathrm{Y}=-\frac{3}{4} \frac{\mu}{\mathrm{D}^{3}} \cdot \frac{k b^{2}}{p^{2} b^{2}-g k} \cdot \cos ^{2}(p t-\phi),
$$

showing that if the depth $k$ of the sea be less than $\frac{p b^{2}}{g}$, 
or be less than 14 miles, there will be low tide immediately under the luminary.

In this instance there was only a semi-diurnal tide, but when the solar path is no longer restricted to the equator, and the channel is any position on the earth, we have both diurnal tides and tides of longer period. The diurnal tide does not exist when either the canal is equatorial, or the luminary is on the equator. When it exists, the phase at the instant of transit depends on whether the depth of the sea be less than $3 \frac{1}{2}$ miles, and the luminary on the opposite side of the meridian or not. If both these hold or both fail, there will be low water at zenith transit; if one fails and the other holds, high water.

There are also tides of long period; that is to say, the relative mean level in different parts of some canals is not the same as if the disturbing body did not act. The mean level thus depends on the position of the luminary.

The state of the tide will manifestly be affected by friction; it appears that if we wish to calculate the height of the tide, at any time $t$, we are to proceed as if calculating the height (on the supposition of no friction) at a later time $t+\alpha$, with the luminaries in the position they were in at a preceding time $t-\beta$, where $\alpha$ and $\beta$ are two quantities given by the theory, and depending on the amount of the friction.

In considering the effects of two bodies, we see that, according to this theory, they do not produce effects which have the same ratio as in the equilibrium theory. If in that theory, $\rho$ be the ratio of the lunar to the solar high tide, and $\rho^{\prime}$ the ratio given by the wave theory, then

$$
\frac{\rho^{\prime}}{\rho}=\frac{n^{2} b^{2}-g k}{n^{\prime 2} b^{2}-g k}
$$

where $n, n^{\prime}$ are the angular velocities of the sun and moon relative to a fixed line on the earth, $b$ the radius of the earth, and $k$ the depth of the sea. This ratio will be different according to the different values of $k$. This explains the fact, that in different seas the ratio of the 
lunar and solar tides is not constant, but varies with the depth of the sea.

It would take too long to enter into all the applications of this theory to oceanic tides. If we could consider the Atlantic as a canal running nearly north and south, the tides in it would be nearly stationary. This is certainly not the case. The South Sea might be considered as a canal running east and west; in that case there would be a forced tide wave travelling along it. The only adrantage of this theory in its application to oceanic tides, is the simplicity of its analytical processes. It is even possible to consider the effect of friction. The seas to which we apply its results are not canals, but we only use them as suggestive of the general characters of the motion.

The theory is most successful in determining the tides in rivers. The water at its mouth being disturbed by the tides formed in the open sea, that disturbance will be propagated, with unaltered period, up the river with a velocity proper to the depth, viz., the square root of product of the mean depth and the force of gravity ( $32 \cdot 18 \mathrm{ft}$.) This disturbance is a long wave; when the top part of the wave passes any point, it forms high water, and when the lower part, low water at that place. The length of the wave of course depends on the distance up the river travelled by the front part of the wave formed at the mouth of the river, while the other parts are being formed. There may even be many high and low tides on the same river at once. Thus La Condamine observed about twenty places on the Amazon where there was high water with low water at the same time at intermediate places. It is also possible, as this long wave is propagated up the river, that the low water at some place a considerable distance up the river may be higher than the high water at the sea. This occurs on the Thames and most rivers.

This propagation of the tide is not the transference of a body of water up the river, but the motion of a form. 
We have seen that when a wave travels, along a uniform channel, that the water is moving in the direction in which the wave travels, so long as the water is above its mean level, and in the opposite direction so long as it is below its mean height. This is also the case with the tide, except so far as this circumstance may be modified by the varying depth, breadth, \&c., of the river. It is a common error to suppose that the flow up the river ceases when the tide begins to fall. The flow in a uniform canal continues for three hours after tide. But if the depth and width be decreasing, if there be any friction among the particles of water, if there be any impediments to the progress of the wave, as a barrier or a series of bridges, the theory shows that this interval will be much less, and observation confirms the result, for the mean interval at which slack water follows high or low tide at Deptford has been shown*. to be thirty-seven to forty minutes.

The height of the tide wave also experiences many alterations due to the varying circumstances of the river. If the tide be stopped by a barrier, or if we are considering the tides in a gulf like the Bay of Fundy, theory shows that there will be tide waves reflected from the barrier, and the height of the tide at the extremity may be greatly increased. If the breadth or depth decrease, the height of the tide will be increased, particularly by any decrease of the former. On entering any river, then, we may expect the tide to be greatly increased. The magnitude of this change depends on the breadth of the wave, and this again depends on whether the tide be formed by the sun or moon; hence the ratio of the tides formed by the two luminaries will not be exactly the same at all places. But when the tide has entered the river, the friction of the particles of water causes the disturbance to diminish in geometrical progression as it goes up the river. "At the entrance of the Bristol Channel the whole rise

* Phil. Trans. 1842. 
at spring tides is 18 feet, at Swansea 30 feet, at Chepstow about 50 feet." "At Newnham it is reduced to 18 feet, and it is still less at Gloucester."

The form of the wave itself is also changed in its progress up the river, if the channel is so shallow that the height of the wave bears a sensible proportion to the depth. It is in this case necessary to carry our solution of the Hydrodynamic Equations to a second or third approximation. When this is done we find that the high water travels up the river with a greater velocity than the phase of low water; the wave becomes steeper in front, and long and gentle in rear. Thus it takes less time for the tide to rise than to fall. "In the St. Lawrence at 40 leagues below Quebec, the rise and fall occupy equal times; at 6 leagues below Quebec, the rise occupies fire hours and the fall seven hours; at twenty leagues above Quebec, the rise occupies three hours and the fall nine hours." The rule given by theory is, that the excess of the time of the water falling over the time of rising is six times the product of the time occupied by the tide wave in passing from the open sea to the station under consideration, and the ratio of the rise of the tide above the mean depth to the mean depth. In this change of form we find an explanation of the bore. Two things, says Professor Airy, are necessary for its formation. There must be a large tide rising with rapidity, and the channel of the river must be bordered with a great extent of flat sands near the level of low water. The tide rises rapidly in the centre of the river, but the water is not broken. But, the rise being very rapid, the water is elevated above the sands faster than it can cover them, and therefore rushes over them with a great velocity and a broken front. As the tide proceeds up the river, a still more extraordinary change of form takes place; the middle of the rear becomes less and less steep; it is at last horizontal, and finally slopes the other way. Thus for every high tide at the mouth there may be two unequal high tides at a distance up the river. In accordance with this, Mr. Russell has observed a double 
tide on the Dee. On going to a third approximation, triple tides are found possible, and this occurs on the Forth. When there are barriers in the river, as the land at the termination of the Gulf of Fundy, the reflected wave is such that there is no horizontal motion at the barrier, and that the tidal elevation at the mouth, caused partly by the sea and partly by the reflected wave, shall altogether be equal to the tide in the sea itself. Hence just as in the case of sound waves in a tube, there will be " nodes" and "loops," if I may so call them. At some places there might be no tide, and at others there will be a rising and falling of the water, but no wave will be propagated along the channel. This is called a stationary tide. The usual case is that which corresponds to the "fundamental node." There will be high water at all parts of the gulf at the same moment, and the tide will be greater the greater the distance from the mouth, and at the termination will be double that at the mouth. But when there is friction in the water, this is not exactly the case. It can be shown that then a tide wave does roll up the river with a velocity different from that proper to the depth, and the end of the flow follows close upon high water. The height of the tide also may or may not increase as it travels along, according to circumstances.

In canals adjoining two tidal seas in which high water does not occur at the same moment, the motion of the water must be found by the same kind of reasoning. But as the result is complicated, we shall not consider this case.

The motion of the tide in channels deeper in the middle than at the sides, as, for instance, the English Channel, has also been considered by Professor Airy. The velocity of the phase is greater in the centre than along the sides. The tide wave will, therefore, become slightly convex, and the velocity being always normal to the front, it will assume such a shape that the part of the normal intercepted between two consecutive positions shall be proportional to the velocity of the wave along that normal. Thus the 
tide curve along the shore will assume a position nearly parallel to the line of coast. The phenomena of the motion in mid-channel will therefore be the same as that in ordinary channels, and the direction of the current will change only when the water is at its mean height. But near the coast there will be a reflected wave from the coast, and the phenomena of motion will more resemble that of the tides in a short gulf; the direction of the current will therefore change at high and low water. At places intermediate, intermediate phenomena will take place. Thus the direction of the current will be intermediate between the direction of the current at the centre of the channel and that along the shore. A little consideration will show that on one side, the current at any spot will, in one complete period from high to low water, go round every point of the compass in the direction of the hands of a watch, and on the other side in the opposite direction. The former phenomena are found to occur on the English side of the Channel, the latter on the French side.

Such are our present theories of the tides. It may be said that we have almost obtained a complete explanation of river phenomena. The great difficulty is with the ocean. In mathematical language we can solve the question of the motion of the tide in one dimension but not in two. Philosophers have been of late very assiduous in collecting the "facts" of oceanic tides, rightly considering that such knowledge will enable us greatly to advance the theory. Many interesting papers on this subject will be found in the Philosophical Transactions, and to these we must refer our readers.

The first step was the construction of what is called "co-tidal lines." These are curves drawn through all the places on the globe which have high water at the same instant. By drawing these lines for the successive hours of the day we can trace the progress of the tide wave over the whole globe. A beautiful map of these will be found in Johnston's Physical Atlas. It appears that the tide wave travels east to west in the great South Seas, as a 
forced wave following the moon. On reaching the Cape of Good Hope, one tide wave is sent up the Atlantic northwards, and another continues its course into the Pacific, and travels westwards and northwards up the west coast of America in a very remarkable manner. The tide wave in the Atlantic travels, therefore, from the south northwards, and arrives at England fourteen hours after it left the Cape. It here sends branches into the English and other channels that environ England, while the chief wave continues its progress northwards, sending other tide waves, travelling from north to south, down the same channels. Two tide waves arrive, therefore, at the mouth of the Thames, and the London tide is compounded of these two. One has travelled along the south coast in seven hours, and the other has gone all round Scotland and descended the German Ocean in about twenty hours. The difference of these intervals is about twelve hours; thus the two semi-diurnal waves arrive in nearly the same phase and strengthen each other, and the two diurnal waves in opposite phases and destroy each other. There is therefore at London no diurnal variation of the tide. These two tides, though they meet at the Thames, do not travel in exactly opposite directions. They both travel a little eastwards, in direct opposition to the motion of the moon. They are both also greatly modified by the many shoals found in those seas. As may be supposed there are points where the two tides meeting in opposite phases, even the semi-diurnal tide will be destroyed. Captain Hewitt has discovered such a point. It is situated about latitude $52 \frac{1}{2}^{\circ}$; there is simply an alternate tidal current, but no elevation of the water.

The tide wave in the Atlantic is probably partly formed in the Atlantic and partly derived from the South Seas. If it were entirely formed in the Atlantic, as the length of that sea from south to north is greater than its breadth, the tide wave would have a tendency to travel partly along its length, with a very irregular velocity, and partly along its breadth. If the tide were entirely derived from the 
Southern Ocean, the tide wave would travel along its length with a velocity proper to its depth. This, as may be supposed, is very irregular. The mean velocity of the tide wave would indicate a mean depth of three and a half miles.

Again, if we suppose that a tide wave would be reflected from the northern coasts of America, and regard the Atlantic as a gigantic closed gulf leading out of the South Sea, the velocity of the tide wave would not merely depend on the depth. Until we know more of the nature of the bottom of the sea, we cannot determine theoretically the motion of the tide wave.

By theory, the diurnal tide wave should be very small near the equator and poles. But this is not the case. The reason is manifest. We hare neglected, in our theory, the effect of the configuration of the coast. The importance of this is manifest from the slight sketch we have given of the progress of the tide over the ocean.

It is impossible for us to do more than merely allude to the effect of wind, shoals, \&c. on the tides. Neither can we enter on the question of aërial tides. That such tides must exist is evident, for the attraction of the luminaries will disturb the air as well as the water. The extent of these oscillations have even been determined by very accurate observations of the barometer. The variations of pressure, as indicated by the barometer, also affect the ocean tides in a remarkable manner. Daussy, Lubbock, and Birt have investigated the amount, and shown that a rise of one inch of barometer will cause a depression of the tides of 7 inches in London, 11 at Liverpool, and 13 at Bristol. 

A P P E ND IX. 



\section{A P PENDIX.}

\section{No. I.-Galileo.}

SoME uncertainty exists respecting the decree or sentence against Galileo, and the Copernican System. The advocates of the Romish Church, indeed, deny that it was pronounced against the doctrine of the earth's motion and the sun's rest, and affirm that the sentence was against Galileo personally, on account of his breach of the promise which he had made to Paul V. (Borghese), and the performance of which he evaded by giving the doctrine in the form of a dialogue. It is also alleged that the sentence is pronounced by the Inquisition, and not by the Holy See. Of this there can be no doubt; but it sets forth a previous "declaration of the theological qualifiers, made by desire of His Holiness, as well as of the Inquisition," that the Copernican system is "absurd and philosophically false and formally heretical, beeause expressly contrary to the Holy Scriptures." This seems to go beyond the mere assertion that it is only false as being unscriptural. There is, however, no doubt that the alleged infallibility of the Holy See is confined to matters of faith; and thus its advocates have some ground for their assertion, that the heretical nature of the doctrine was alone set forth both in the sentence on Galileo and in the previous proceedings. The supposition that he was subjected to 
torture in order to obtain his recantation, appears to rest on no foundation, except the use of the terms rigorous examination (rigoroso esame), said to be the form in which torture is referred to by those sentences. But the story is completely negatived by Galileo's own letter: giving an account of the manner in which he had been treated, acknowledging the respect paid to him, and the lenity of his imprisonment, or rather nominal detention, in his friend the Archbishop of Pisa's (Picolomini's) palace. (Tiraboschi, Lett. 1tal. tom. viii.* lib. ii. p. 147.) It is probable that the story of his whispering to a friend "E pur si muove," ("And it moves for all that,") when he rose from his knees, on which he had made the recantation, rests upon no better foundation. Nothing can be more unlikely, at least, than his choosing such a moment for this pleasantry - a moment of great though forced humiliation, when he had in the most solemn manner called God to witness, that " he abjured, cursed, and detested" the positions which he entirely believed; nothing more unlikely than his exposing himself to the risk of his words being discovered by immediate examination of the person whom he addressed.

But it seems still somewhat doubtful how far the sentence upon Galileo has been reversed. His "Dialogue" had by decree of the "Congregation of the Index" been put into that list of forbidden books; and Leo XII. (Genga) nearly thirty years ago ordered it to be expunged from that list. Mr. Drinkwater (Life of Galileo, p. 64. $\%$ ) states that it had not been erased in 1828. Mr. Lyell (Principles of Geology, p. 56, edition, 1853), considers that this assertion is inconsistent with the account which he received from Professor Scarpellini at Rome, in 1828,

* Viviani, who is described as devoted to Galileo more than a son to a father, and who attended his master for the last three years of his life (Montucla, ii. 290.), "gave not the least countenance to the exaggerated accounts of his treatment.

† Published by the Useful Knowledge Society in 1829. 
that Pius VII. (Chiaramonte) had assembled the Congregation "which repealed the decree against Galileo and the Copernican system," with only one dissentient voice. It is, however, possible that the Index might still retain the name of the book. It must also be remembered that nothing can be more inconsistent with the usual proceedings and policy of the Roman See, than making such an admission of error as is involved in the repeal of a sentence pronounced, and purporting to proceed upon the ground of heresy. There can be no other explanation given of such a man as Benedict XIV. (Lambertini), the friend and patron of Boscovich, the correspondent of Voltaire and other literary men, himself an eminent cultivator of letters, and remarkable for the liberality of his opinions, suffering the sentence on Galileo to continue the disgrace of his church.

It must, however, be borne in mind, that probably soon after Galileo's condemnation, certainly for the last century or more, the prohibition to teach the Copernican system was little more than nominal; nothing else was required than to term it the Hypothesis, and not the doctrine, or theory, of the earth's motion. The declaration of the Fathers (Leseur and Jacquier) accordingly calls it an $\mathrm{Hy}$ pothesis. They regard the proceedings in Galileo's case, (to which it is manifest that their words refer) as a decree against the motion of the earth. But many have suspected that this is a covert attack on those proceedings; and certainly, considering the time of the declaration appearing, when Benedict XIV. was in every way promoting science and letters, and pursuing generally the most liberal and enlightened policy, this supposition is not without plausibility.-It must be added that whenever the outward deference to the decree of the Consistory was shown, the doctrine, under the name of Hypothesis, was openly taught, with the utmost freedom in giving the proofs whereon it rested. 
No. II. (p. 58).-Another Demonstration. TAKING the expression $\frac{\mu}{r^{2}}$ for the force and making it $=$ the general form $\frac{h^{2} r}{p^{3} \mathrm{R}}$ where $h$ is constant, we have $\frac{h^{2} r}{p^{3} \cdot \mathrm{R}}=\frac{\mu}{r^{2}}$. This is a differential equation of the second order, and it can have but one general integral, and that can contain only two arbitrary constants. Such an integral has been found; for it has been shown that any conic section with the focus in the centre of force satisfies the equation. The nature of the case shows that any singular solution is out of the question. We may also arrive at this result by reversing the process in p. 49. Substituting for $R, \frac{h^{2}}{p^{3}} \times$ $\frac{d p}{d r}=\frac{\mu}{r^{2}}$ and $\frac{d p}{p^{3}}=\frac{\mu}{h^{2}} \frac{d r}{r^{2}}$, and integrating, $\frac{1}{p^{2}}=\frac{2 \mu}{h^{2}}$ $\left(\frac{1}{r}+\mathrm{C}\right)$. This is a well known property of all conic sections; but to show that no other curves possess it, put $\frac{1}{r}=u$; then since $\frac{1}{p^{2}}=u^{2}+\overline{\frac{d u}{d \theta}}^{2}$, therefore $\left.\frac{\overline{d u}^{2}}{d \theta}\right|^{2}+u^{2}=$ $2 \frac{\mu}{h^{2}} \cdot u+\mathrm{C}$, and therefore $\frac{d u}{\sqrt{\mathrm{C}+\frac{2 \mu u}{h^{2}}-u^{2}}}=d \theta$. 
The integral of this, calling $\mathrm{C}+\frac{\mu^{2}}{h^{4}}=\mathrm{L}^{2}$, is $\cos ^{-1} \frac{u-\frac{\mu}{h^{2}}}{\mathrm{~L}}$ $=\overline{\theta-\alpha}$, or $\frac{1}{r}=u=\frac{\mu}{h^{2}}+\mathrm{L} \cos \overline{\theta-\alpha}$, the general equation to a conic section.

\section{No. III.-Force VARYiNg INVERSELY as the DISTANCE.}

IT is remarkable that what at first sight seems to be the most simple of all the cases, that of the central force varying inversely as the distance, or of $m=1$ in $\frac{\mu}{r^{m}}$, should be found so much the most difficult of solution, and that, whether the proportion of $\frac{1}{r}$ enters into motion related to one centre only or to more centres than one. Herman, in the Phoronomia, turns away from it, merely observing that his formula fails when $m=1$. Clairaut, in his excellent commentaries on the Principia, his additions to Madame du Chatelet's translation, deduces, chiefly from the Propositions of the Second and Eighth Sections (Lib. I.), a general differential equation for the curve described by a body under the influence of a centripetal force as $Y$, a function of the radius vector; and the equation is therefore a polar one. It involves the integration of $f \mathrm{Y} d y$. Consequently, when $\mathrm{Y}=\frac{1}{y}$, the case we are now considering, the integral contains an unmanageable logarithm; 
for the equation becomes $b^{2} f^{2} d x=\frac{d y}{y\left(2 \mathrm{~B}-2 \int \frac{d y}{y}\right)^{\frac{1}{2}}}$ $=\frac{d y}{y^{2}\left(2 \mathrm{~B}-\log y^{2}\right)^{\frac{1}{2}}}$. He makes no mention of this case, as, like Herman and most others, he seems unwilling to approach it ; undoubtedly, however, such is the application of his formula.

Keil, in his paper on Central Forces in Phil. Trans. 1708, p. 174., gives the case of the force as $\frac{1}{r}$ and reduces it to finding $\frac{d}{(b-\log r)^{\frac{1}{2}}}=\mathrm{P}$, the perpendicular to the tangent.

By one process grounded on Prop. XLI. Lib. I., this result is obtained for the case of $\frac{1}{r}$, that is $\frac{1}{\left(x^{2}+y^{2}\right)^{\frac{1}{2}}}$ $\iint \frac{2 d x d^{2} x+2 d y d^{2} y}{d t^{2}}=\int 2 \mu \frac{(x d x+y d y)}{x^{2}+y^{2}}$, or $\int \frac{d x^{2}+d y^{2}}{d t^{2}}-2 \log \left(x^{2}+y^{2}\right)-c=0$; and $d t^{2}$ being $=\frac{(y d x-x d y)^{2}}{c^{2}}$, the equation becomes $\int \frac{d x^{2}+d y^{2}}{(y d x-x d y)^{2}}$ $-\frac{\mu}{c^{2}} \log \left(x^{2}+y^{2}\right)+\frac{v}{c^{2}}=0$.

The process grounded on the formula $f=\frac{h r}{2 p^{3} \cdot \mathrm{K}}$ is, if possible, more hopeless; for this gives

$$
\begin{aligned}
& \frac{h\left(y^{2}+(x-c)^{2}\right)^{\frac{1}{2}} \times\left(d x d^{2} y-d y d^{2} x\right)}{2(y d x-x d y+c d y)^{3}} \\
= & \frac{1}{\left(y^{2}+(x-c)^{2}\right)^{\frac{1}{2}}}, \text { or } h\left(y^{2}+(x-c)^{2}\right)\left(d x d^{2} y-\right.
\end{aligned}
$$


$\left.d y d^{2} x\right)=2(y d x-x d y+c d y)^{3}$, or $\frac{h d y}{d x}=$ $\int \frac{2(y d x-x d y+c d y)^{3}}{d x^{2}\left(y^{2}+(x-c)^{2}\right)}$.

The difficulty follows $\frac{1}{r}$ wherever that proportion enters into the investigation. Thus in the problems connected with different centres, when it is found that forces varying as $\frac{1}{r^{2}}$ and $\frac{1}{q^{22}}$, being combined with forces varying as the distance directly, or as $r$ and $q$, give an elliptic orbit, the resultant of the latter forces passes through the centre, and the locus of that resultant is the opposite semi-ellipse, and so of a circle. But when the proportion is $\frac{1}{r}$ and $\frac{1}{q}$, (also if the force towards each centre is as the radius vector to the other centre, ) the resultant passes through innumerable points to an opposite curve, sometimes of a different kind, although each resultant differing in its direction from all the others, and in the case of the circle, from the diameter, is equal to the one passing through the middle point of the line joining the two centres. In this case, therefore, there is no combined action of the forces $\frac{1}{r^{2}}$ and $\frac{1}{q^{2}}$, or $\frac{1}{r^{5}}$ and $\frac{1}{q^{5}}$ or of their several resultants, with the resultant of $\frac{1}{r}$ and $\frac{1}{q}$, as there is in the case of $\frac{r}{m}$ and $\frac{q}{m}$, but the several forces act wholly in the direction of the radii vectores severally.

It evidently appears to be a more simple and natural combination that the two sets of forces should diminish 
with the distance increasing, as in $\frac{1}{r^{2}}$ and $\frac{1}{q^{2}}$ combincd with $\frac{1}{r}$ and $\frac{1}{q}$, than that one set should decrease and another increase with the distance, as in $\frac{1}{r^{2}}$ and $\frac{1}{q^{2}}$ with $r$ and $q$, in which case there must even be an extinction of force at one point, where (taking the sum of the forces instead of their resultant) $\frac{m}{r^{2}}+\frac{m}{q^{2}}=\frac{r+q}{m}$, or $r$ is as in the equation $\cdot r^{3}+\frac{q^{3}-m^{2}}{q^{2}} r^{2}=m^{2}$. Of course the value of $q$ would be the same; and the resultant (more accurately taken to measure the increase of the force) would at one value give the two sets of forces as counterbalanced.

The younger Euler (J. A. Euler) has a paper in the Berlin Mem. 1760, p. 250, upon the action of a central force decreasing as the distance, in the case of the attracted body's descent towards the centre, and states the reason of this problem being insoluble except by arcs or logarithms. He finds that taking $a=$ the height from which the descent begins, $f=$ that at which the centripetal force is equal to the gravity of the attracted body, the time of descent towards the centre is $=\frac{1}{\sqrt{f}} \int \frac{d y}{\sqrt{\log \frac{a}{y}}}, y$ being the distance from the centre.

No. IV.

CENTRAL FORCES TO MORE THAN ONE POINT.

1. IT is to be lamented that Sir I. Newton did not treat the problem of forces directed to more fixed points than 
one, as to two such points, either in the same or different planes from the body acted on. This is the fundamental point in considering disturbing forces when the centres are not fixed, which makes the problem more complicated and difficult. It is, however, sufficiently so even where the centres are fixed.

2. That the subject must have attracted his attention there can be no doubt. He had gone so much into the more difficult inquiries respecting disturbing forces that he must have fully considered the somewhat simpler; what may be termed the fundamental, case of fixed centres. Indeed, a paper communicated to the Royal Society in 1769 (Phil. Truns. p. 74.) contains a demonstration by W. Jones, an intimate friend of Newton, of a proposition on this subject, which Machin had immediately after Sir Isaac's death given to the translator of the Principia. Machin had observed on the want of some investigation of the motion of forces directed to two centres, as required to explain the motions of planet and satellite, which gravitate to different centres, in a word the problem of the Three Bodies. The proposition of Machin and Jones goes but a very little way to supply the defect complained of. It is confined to the case of the line joining the two centres being in different planes from the line of projection; it is that the triangle formed by the radii rectores and the line joining the two centres or fixed points, describes equal solids in equal times round that line; and the demonstration is similar to that of the first proposition, of equal areas in equal times when a single force is directed to one centre. It seems reasonable to conclude, that Newton had, upon full consideration, found the full investigation of the subject beyond the powers of the calculus as it then existed. It is at least certain that, though he might have mastered it, he never could have delivered his results synthetically as in the Principia.

3. The solutions on disturbing forces generally consider one force as acting in the one direction, that of the radius 
vector, and another in a line perpendicular to that radius vector. Thus Clairaut (Mem. Acad. 1748, p. 435.) gives these equations $r d^{2} v+2 d r d v=\Pi d x^{2}$

$$
r d v^{2}-d^{2} r \quad=\Sigma d x^{2}
$$

$v$ being the radius vector, $v$ its angle with the axis, $d x$ the differential of the time, $\Pi$ the force to the centre, $\Sigma$ the disturbing force. So D'Alembert (Mem. Acad. 1745, p. 365.) takes the same course, and obtains an equation to the orbit in question, depending on the integration of $\int \Pi d z$, $\Pi$ being the disturbing force acting in a line perpendicular to the radius vector, and $z$ the circular arc described with a radius equal to the distance between the centre of force and the vertex of the orbit. This assumes, however, that the orbit is itself nearly circular.

4. If $\mathrm{P}=$ distance of $\mathrm{E}$ (Earth) from Moon (M)'s quadrature, $s=$ sin. angle of rad. vec. $r$ with the perpendicular to $a$, the distance of $\mathrm{E}$ from $\mathrm{S}$, the Sun;

$v=$ velocity of $\mathrm{M}$; then $v d v=\frac{r d \mathrm{P}}{\mathrm{P}}+\frac{3 \mathrm{P}^{2} m n s d s}{a^{3}}$,

supposing the motion of $\mathrm{M}$ to be almost uniform. Here one of the forces acting on $\mathrm{M}$ is directed towards $\mathrm{E}$ and is $=\frac{E+M}{M E^{2}}+\frac{S \times M E}{S M^{3}}$; the other force is in a line parallel to $\mathrm{SE}$ or $a$, and is $=\frac{\mathrm{S} \times \mathrm{SE}}{\mathrm{S} \mathrm{M}^{3}}-\frac{\mathrm{S}}{\mathrm{SE}^{2}}$. It was in consequence of this investigation that Clairaut for some time announced, as did also Euler and D'Alembert, that there was a material error in the Newtonian theory of the Moon's motion. The error, which afterwards was found to arise from their having omitted the consideration of certain quantities, was acknowledged by Clairaut three sears later (Mem. Acad. 1748, pp. 421.434.), but no one 
can read that paper without feeling that the acknowledgment was too coldly made, after he had gone so far as to suppose that the whole Nertonian doctrine was overthrown, and to propose a new law of $\frac{1}{r^{2}}+\frac{1}{r^{4}}$, the whole of this arising from his own error. It is to be remarked, however, that the investigation of 1745 was in all respects most accurately conducted, and must have led to the same result as in 1748 but for the supposition that certain quantities might safely be neglected. Even in 1745 Clairaut, upon Newton's assumption of the excentricity of M being nothing, comes to his conclusion that the proportion of the axes is as 69 to 70 .

4. Legendre treats the subject very fully, as far as regards two centres, and also confining himself to the forces being inversely as the square of the distance (Exercises de Calcul. Integral. part iv. sect. 2.). He deduces from his analysis several theorems, two of which he regards as very remarkable. The first apparently strikes him in this light, because it shows the same orbit to be produced by the combined action of the two forces directed towards two foci, as either force would produce acting on the body, and directed to one of the foci. If $\mathrm{V}$ is the velocity at the vertex of the ellipse which would make the body describe that curve when acted upon by the force directed to one focus, $v$ the relocity at the same point which would make the body describe the ellipse when acted upon by the other force directed to the other focus; then if the two forces act together upon the body, and $I$ is the initial velocity, or velocity of projection, it will describe the same ellipse, provided $\mathrm{I}^{2}=\mathrm{V}^{2}+v^{2}$.

5. The other theorem follows from his integration which gives the expression for the time. It is that if two equal forces act upon the body directed to the two foci, and the masses of the attracting bodies consequently are equal, the revolving body will describe the ellipse in a shorter peri- 
odic time, will move more swiftly, than if the whole mass were placed in one focus and acted from thence upon the revolving body. He takes the example of the tangent of the orbit with the axis making an angle of $30^{\circ}$, and finds the periodic time shorter in the proportion of nearly 78 to 100 when the attracting mass is divided into two, one acting in each focus, than when both combined act from one focus,

6. What renders this problem of more centres than one so difficult, is that the resultants of the forces pass through different points, and that they vary by a law which differs in each case, as the locus of their extremities is a different curve. Take the least complicated, but still full of difficulty, that of two fixed points as the centres of force, and take the instances in nature of the forces being inversely as the square of the distance; the radius vector to one point being $r$, the force $\frac{m}{r^{2}}$; to the other point the radius vector $q$, the force $\frac{m}{q^{2}}$. Now the force which acts on the body being the resultant of these two, and these forces not being as $r$ and $q$, the diagonal does not pass through the middle point of the line joining the two centres; except in the single point of the orbit where $r=q$, and even then it may not reach that line, for it is $\frac{\sqrt{2} \cdot m}{r^{2}}$. At every other point it runs in a different direction. Let $S$ and $S^{\prime}$ be the two fixed points; $\mathrm{S} \mathrm{P}=r$, and $\mathrm{S}^{\prime} \mathrm{P}=q$. Then $\mathrm{P} a$ being taken $=\frac{m}{r^{2}}$, and $\mathrm{P} a^{\prime}=\frac{m}{q^{2}}$, the resultant at that point $\mathrm{P}$ bisects $a a^{\prime}$ and is $\mathrm{P}$, and produced, $\mathrm{P} \mathrm{M}$ cutting the axis. From hence may be seen how complicated would be the analysis, how next to impossible the geometrical construction of the locus of $\mathrm{P}$, by referring the lines $\mathrm{PM}$ to 
$\mathrm{SS}^{\prime}$ as an axis. We know indeed that one of the forces $\frac{m}{r^{2}}$ or $\frac{m}{q^{2}}$ acting towards $\mathrm{S}$ or $\mathrm{S}^{\prime}$, the locus of $\mathrm{P}$ is an ellipse ;

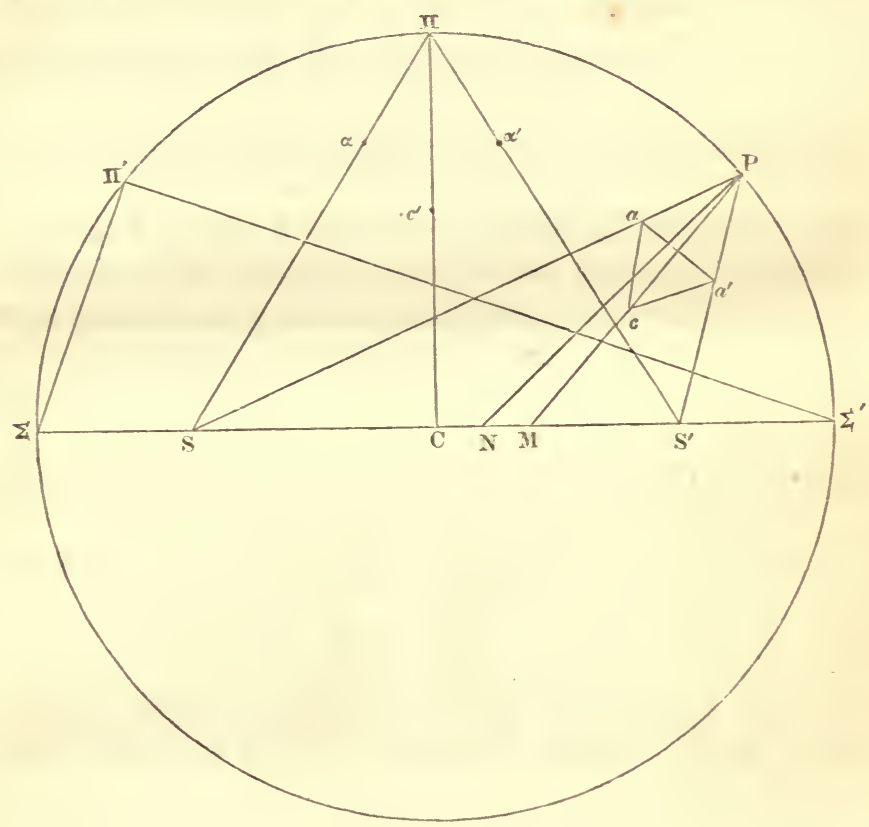

but it would not follow that if both forces acted the same curve would be the locus. That the force would be different is certain, because it would be as $\mathrm{P} c$, and not as either $\mathrm{P} a$ or $\mathrm{P} a^{\prime}$. But it may be said that the curve also would be different. Let us, howerer, suppose the case of the curve, whatever it be, cutting the axis $\mathrm{S} \mathrm{S}^{\prime}$ produced at $\Sigma$ and $\Sigma^{\prime}$, points equally distant from $S$ and $S^{\prime}$, so that $S \Sigma=S^{\prime} \Sigma^{\prime}$; also that the angle and the initial velocity of projection from $\Sigma$ and $\Sigma^{\prime}$ is the same, and further that the 
attraction as the mass is the same from $S$ and $S^{\prime}$, or that the mass of the body in $S$ and in $S^{\prime}$ is the same; then it seems impossible to avoid the conclusion that an ellipse, and the same ellipse, must be described; because one of the forces alone acting from $\mathrm{S}$, as $\frac{1}{r^{2}}$, would give the ellipse passing through $\Sigma$ and $\Sigma^{\prime}$; and the other force alone acting from $\mathrm{S}$, as $\frac{1}{q^{2}}$, would give the ellipse passing through the same points $\Sigma$ and $\Sigma^{\prime}$; and the initial velocity * and angle of projection would prevent any difference in the length of the conjugate axis; and in the middle point answering to the centre $\mathrm{C}$, the equality of $r$ and $q$ and of $\mathrm{P} a$ and $\mathrm{P}^{\prime}$ would make the diagonal $\mathrm{P} c$ coincide with the conjugate axis. But a further combination of forces may be supposed in this case; two forces acting towards the points

$\mathrm{S}$ and $\mathrm{S}^{\prime}$ and in the proportion of $r$ and $q$, or $\frac{r}{m}$ and $\frac{q}{m}$.

How will this addition affect the locus of $\mathrm{P}$ ? It should scem, for a reason similar to that before given, that the curve would remain the same; for the two new forces

$\frac{r}{m}$ and $\frac{q}{m_{\imath}}$, acting in $r$ or $q$ or $\mathrm{PS}$ and $\mathrm{PS}^{\prime}$ respectively,

their resultant must, if there were none other acting, pass through the middle point $\mathrm{C}$, between $\mathrm{S}$ and $\mathrm{S}^{\prime}$; and as we know that a force acting from that point, and in proportion to the distance from that point, causes the body to move in an ellipse whose centre is that point, and $r+q$ being.constant, the ellipse must have the same axis and coincide

* The condition of Legendre (mentioned in page 427 ), that $\mathrm{I}^{2}=\mathrm{V}^{2}+v^{2}$ is supposed to hold; for otherwise the centrifugal force would not be sufficient to balance the centripetal. 
with the ellipse produced by the combination of the forces $\frac{m}{r^{2}}$ and $\frac{m}{q^{2}}$.

7. This had appeared to be a necessary consequence of the conditions stated, but not as at all proving the velocity to be the same in the ellipse, when described by one force $\frac{m}{r^{2}}$ or $\frac{m}{q^{2}}$, or when described by the combined action of both, or when described by the combined action of $\frac{r}{m}$ and $\frac{q}{m}$, or of $\frac{m}{r^{2}}, \frac{m}{q^{2}}, \frac{r}{m}$, and $\frac{q}{m}$; because in all those cases the velocity will be different, and particularly the action of $\frac{m}{r^{2}}+\frac{r}{m}$ with $\frac{m}{q^{2}}+\frac{q^{7}}{m}$ will occasion adifferent velocity in each point from that occasioned by $\frac{m}{r^{2}}+\frac{m}{q^{2}}$. Thus to take the velocity at one point answering to $C$. If $\Pi \alpha$ and $\Pi \alpha^{\prime}$ be taken as $\frac{m}{r^{2}}$ and $\frac{m}{q^{2}}$, the diagonal $\Pi c^{\prime}$ is the force of $\frac{m}{r^{2}}$ and $\frac{m}{q^{2}}$ combined, $\Pi C$ is the resultant of $\frac{r}{m}$ and $\frac{q}{m}$ combined (supposing $m=1$ ). Therefore the velocity in $\Pi$ will be as $\Pi c^{\prime}+\Pi \mathrm{C}$, when all the forces act, and only as $\Pi c^{\prime}$, when the two former act alone, and as $\Pi \mathrm{C}$ when the two latter act alone.* But the curves appear to be the same in each case.

8. These consequences seeming to follow from a con-

* The difference in velocity is easily obtained, in comparing the effect of one force and of the combined forces, from the equation $v^{2}=\Sigma(f \times$ half chord osculating circle, the chord being $=\frac{2 p . \mathrm{R}}{r}, p=$ perpendicular to the tangent, and $\mathrm{R}=$ radius of curvaturc. 
sideration of the conditions stated, but without a full and rigorous investigation, it was very satisfactory to find that Lagrange had arrived at the same conclusion in one case of his solution of the problem of two fixed centres (Mcc. Anal. pt. ii. sect. 7. ch. 3.) That solution is marked throughout with the stamp of his great.genius. Euler had, in the Berlin Memoirs for 1760, treated the case of the inverse square of the distance and the centres and orbit being in the same plane. Lagrange's solution is general for the force being as any function of the distance, and of $x, y, z$, being the coordinates. Pressed by the great difficulties of the problem, and the impossibility of a general solution, he first confines himself to the inverse square of the distance (p. 97.), and a general integration being still impossible, even after obtaining a differential equation with the variables separated, he makes a supposition which enables him to obtain two particular integrals (p. 99.), and this gives for the orbit an ellipse in the one case and an hyperbola in the other, with the foci in the two centres of force; and it follows, he observes, from the investigation, that the same conic section which is described in virtue of a force to one focus, acting inversely as the square of the distance, or to the centre and acting in the direct ratio of the distance, may be still described in virtue of three such forces ("trois forces pareilles*"), tending to the two foci and to the "centre." Ho adds: "ce qui est très remarquable" (p. 101.). It having appeared to many persons that a portion of the demonstration was not so rigorous as might be desired, M. Serret has very ably and satisfactorily supplied the defect (Mec. $A n$. tom. ii. note iii. p. 329. ed. 1855), but he arrives at the same

* It is plain that "pareilles" does not mean lof the same kind as $\frac{1}{q^{2}}$ and $\nu$; for he resolves the force to the centre into two acting to the foci, and calls the whole forces $\frac{\alpha}{r^{2}}+2 \gamma r$ and $\frac{\beta}{q^{2}}+2 \gamma q$. 
result. There is also given a very important generalisation of Lagrange's solution, and of Legendre's theorem already mentioned, by M. Ossian Bonnet. (16id. note iv.)

9. The same reason already given proves that if, instead of two points not in the trajectory we take two in it, as $\Sigma$ and $\Sigma^{\prime}$, and refer the forces to those two, and make the forces $\frac{m}{r^{5}}$ and $\frac{m}{q^{5}}$ in $\Sigma \Pi^{\prime}$ and $\Sigma^{\prime} \Pi^{\prime}$ respectively, and the angle of projection and initial force the same, the same circle will be described by the body; and that if two other forces also act on it, as $\Sigma \Pi^{\prime}$ and $\Sigma^{\prime} \Pi^{\prime}\left(\right.$ or $\frac{r}{m}$ and $\frac{q}{m}$ ) the same circle will be described by the joint action of the forces. This is even a more remarkable consequence than the other; because the forces acting to the centre would of course give a uniform motion, and those acting to the points in the circumference an accelerated motion, and the forces combined will give an accelerated motion. At the middle point $\Pi$, the velocity will be, if only the forces $\frac{m}{r^{5}}$ and $\frac{m}{q^{5}}$ act, as $\frac{\sqrt{m}}{2 a^{2}}$ if the forces $\frac{r}{m}$ and $\frac{q}{m}$ also act, it will be as $\sqrt{\frac{m}{4 a^{4}}+\frac{2 a}{m}}$. It must, however, be addled, that Lagrange's solution does not contain this case, of the circle and two points in the circumference, and there is very great difficulty in applying to it his analysis. Indeed, it appears that if the problem be worked upon the datum of $\mathrm{R}=\frac{\alpha}{r^{5}}+2 \gamma r$, and $\mathrm{Q}=\frac{\beta}{q^{5}}+2 \gamma q$, there is no possibility of obtaining an expression freed from the integral sign $(\mathcal{C})$ in the same way as Lagrange does from his equation, founded upon the datum $\mathrm{R}=\frac{\alpha}{\gamma^{2}}+2 \gamma r$ and $\mathrm{Q}=\frac{\beta}{q^{2}}$ 
$+2 \gamma q ; m=-2$, and consequently $m+2=0$ seems necessary to his process.

There seems reason to suppose that the kind of reasoning on which we have relied as to the identity of the trajectories had influenced Legendre in confining his investigation to the case of curves which have not infinite branches. $\mathrm{He}$ expressly says (Ex. de Calc. Int. 11. 372.), that he confines himself to curves where the orbit is restricted to a definite space. Certain it is, that the reasons applied to the identity in the case of curves returning into themselves is wholly inapplicable to curves having infinite branches.

10. The extreme complication of the problem arising from the resultants passing through innumerable points in the axis has been above noted, as regards the case of two forces only $\frac{m}{r^{2}}$ and $\frac{m}{q^{2}}$. When we add the other two $\frac{r}{m}$ and $\frac{q}{m}$ the complication is not considered by Lagrange to be increased (p. 99.), and probably it is not as regards the analytical investigation. But it certainly is increased as regards the geometrical construction; for we then have to take the resultant of $\mathrm{P} c$ with $\mathrm{P} \mathrm{C}$ (which is the resultant of $r$ and $q$ ), and this will carry the ultimate diagonal representing the whole force applied to $\mathrm{P}$ beyond the axis $\mathrm{S} \mathrm{S}^{\prime}$. Lagrange indeed does not take $\mathrm{P} \mathrm{C}$ into his analysis, because he supposes the forces $r$ and $q$ to act in the same line of the radii vectores with the forces $\frac{m}{r^{2}}$ and $\frac{m}{q^{2}}$. But this would cause these radii vectores to be produced, and make their resultant also fall below the axis. It can hardly be doubted that these considerations weighed with Sir Isaac Newton, in disinclining him to the investigation of a problem which could afford no hope of a geometrical, or of any synthetical solution. That he had deeply considered the subject of attraction to various 
centres, in the more difficult case of moveable centres is certain. The justly celebrated LXVIth proposition of the First Book affords ample proof of it; and indeed the LXIVth proposition comes so near the subject of this note, that it may be correctly said to contain the grounds both of Clairaut's and Legendre's more full investigation.

11. In connection with this subject Lagrange expresses great admiration of a theorem of Lambert, which no doubt is remarkable, that in ellipses (the central force being as $\frac{1}{r^{2}}$ ) the time taken to describe any arc depends only on the transverse axis, the chord of the arc, and the sum of the radii vectores at its extremities. We may observe, in passing, that the vanishing of the expression for the conjugate axis in some fundamental formulæ connected with the ellipse, for example, the subtangent, gives rise to other curious properties of the curve similar to the one noted in this theorem, which is itself related to that peculiarity. (See a porism arising from this circumstance; Life of Simson, p. 154.) The same theorem had occurred to Lagrange himself, in examining the problem of deflecting forces to two centres; it is indeed derivable immediately from the case of that problem when one force vanishes and the centre connected with it is in an arc of the ellipse; for then the radius vector belonging to that centre becomes the chord. But Euler, long before either of them, in 1744, had given the theorem for parabolic arcs, which they only extended to elliptic arcs, and had published it in the Berlin Mem. 1760. Yet when Lambert claimed it as his own in 1771, and Lagrange gave him the honour of it in 1780, Euler, though he lised three years after, never thought of reminding them of his prior claims. It was thus, too, with the first of analysts, respecting the extension of the Differential Calculus to that of Partial Differences (Life of D'Alembert, p. 466.), by far the greatest step in mathematical science which 
has been made since the age of Newton and Leibnitz, if it have not a rival in the calculus of variations, the honour of which also is shared by him with Lagrange.

12. It must be observed that when in 1771 (Berlin Mem.) Lambert extended the theorem to elliptic arcs, he was ignorant of Euler having anticipated him as to parabolic arcs. But Lagrange truly states (Mec, Anal. ii. 28., ed. 1855), what shows that all of them had been anticipated by Newton. For in the IV. and V. Lemmas of the Third Book he had very distinctly given the whole materials of the proposition as far as parabolic arcs are concerned.

Lagrange notes the uses of the theorem, and observes upon the remarkable circumstance of the time not depending at all on the form of the ellipse, provided the transverse axis remains the same. This must have frequently recurred to his recollection, when engaged in those great investigations which show the connection that the transverse axis remaining unchanged, has with the permanency of the system.

13. He further remarks upon another consequence of the conjugate axis, or the form of the orbit, not affecting the time; namely, that the conjugate wholly disappearing, and the orbit becoming rectilinear, the theorem applies to the time of falling to the centre, on the centrifugal force or that of projection ceasing to act. (Berlin Mem. 1778.) But Newton's VIth Lemma, to which he does not refer, in some degree anticipated this also.

14. The great difficulty of the problem of several centres, has been stated. Euler was clearly of this opinion, and he was the first that undertook the solution. After speaking of the general problem (Berlin Mem. 1760, p. 228.) as alike important and difficult, he confines himself to the case of two bodies in fixed positions, acting upon a third, which moves in the plane of those disturbing bodies; in a word, to the motion of a body drawn towards two fixed centres. He says that, whoever undertakes the 
solution of this less difficult problem "will find difficulties almost as insurmountable as in the great fundamental problem of astronomy ; " and adds that, after making many fruitless attempts, he had at last been led to a solution by the accident of an error into which he had fallen in his investigation. What he proposes is to find the cases in which the curve is algebraical ; there being, according to the conditions, an infinite variety, most of them transcendental. He considers, however, that if this case of two bodies in fixed centres, and in the same plane with the body attracted, should be incapable of solution, the general problem must prove still more so. Nothing can exceed the clearness of his investigation; and the ingenious subtlety of the contrivances by which he facilitates the reduction of his differential equations to those of a lower degree. Of this Lagrange expresses great admiration, who, in giving a solution of the case in some respects more extended, but in others less, became fully sensible of the diff.culties of the process, and whose investigation is less luminous than his great predecessor's. Euler reduces his investigation to the integration of the equation

$$
\frac{\mu d x}{\sqrt{x+x^{3}}}=\frac{\nu d y}{\sqrt{y+y^{3}}} ;
$$

and obtaining the relation between the angles made by the two radii vectores with the axis. It is clear that $\mathrm{La}-$ grange's solution is obtained by another course altogether.

No. V.-Leibnitz's Dynamical Tracts.

EArLY in 1689, about a year and a half after the publication of the Principia, there appeared in the Acta Eruditorum, of Leipsic, two papers of Leibnitz, entitled, "G. G. L. Schediasma de Resistentia Medii et Motû projectorum gravium in medio resistente," and "Tentamen 
de Motuum Coelestium Causis." As these tracts coincide in their subjects and in many of their doctrines with the propositions of Sir I. Newton, it has been held by some, and apparently by Sir Isaac himself, that their author had obtained from those propositions the substance at least of his own. This is certainly a grave charge against Leibnitz; inasmuch as he affirms that he had not seen the Principia when he wrote his two papers, admits that he had seen an abridgment of it in general terms, and expresses regret at not having seen the work itself. $\mathrm{He}$ further states his inability to comprehend by what methods the most important of Newton's discoveries, as described in the abridgment, the elliptical motion of the heavenly bodies, is demonstrated mathematically from the data obtained by observation, and the law of attraction governing that motion; a proposition which Leibnitz gives as deduced by himself with the aid only of the differential calculus. The whole statement, though not in direct terms, yet by manifest implication, represents that he had made his investigation, and arrived at the result, without any further knowledge of the Principia than the fact of Sir I. Newton having obtained the same result by his process, whatever it might be.

It cannot be denied, therefore, that the strongest proof is required to authorise the belief of his having seen the Principia, and borrowed his propositions from thence. But instead of proofs there appear to exist only certain suspicious circumstances. His statement (Act. Erud. 1689, January, p. 36.) is perhaps too particular in describing his absence from home on his official journey. Newton says that a copy of the Principia had been given immediately after its publication, to Facio Duiller, a young mathematician, a friend of Huygens and of his own, for the purpose of its being sent to Leibnitz; but there is no evidence that it reached him. (Macclesfield MS. Paper in Newton's handwriting. Rigaud's Historical Essay, App. Nos. XIX. XX.) The same paper of Newton 
charges Leibnitz with having endeavoured to appropriate Mouton's discovery by denying that he had seen his work before he made the discovery himself.*

It must unfortunately be added, that Leibnitz's conduct in the controversy relating to the invention of the calculus, leaves an unfarourable impression. There was great disingenuousness, to give it no harsher name, in John Bernouilli's proceedings, by the confession of his own family; and Leibnitz, who had encouraged him, betrayed the secret confided to him of his authorship, for the mere purpose of grasping at an advantage, by means of the authority which Bernouilli's great name in the mathematical world gave to his decision against Newton, whom he had opposed by anonymous writings to please his patron. All this, however, we must admit, only affords ground for entertaining suspicions; and the proof required must be sought for in the internal evidence of the works compared together.

The fact of the abstract having appeared in the same work the June preceding is admitted by him, as is his having read it. The account, however, which it contains of the Principia is exceedingly general; none of the investigations are given of the propositions which it states that the work enunciates. We can only consider it as showing that the truths of which it gives a concise summary, are proved by the application of mathematical reasoning to the known phenomena; and a person so learned in this science as Leibnitz could not have read that the Principia treated of the descriptions of various trajectories, particularly the conic sections, according to various data (juxta varia data, p. 308.), without perceiving at once that this must refer to dynamical considerations. But especially must he have perceived in what manner Newton had conducted his investigations, when he found it stated (p. 310.) that the heavenly

* This was probably Mouton's method of interpolation for places between those calculated, instead of proportional parts. 
motions were explained by the propositions in the three first sections of the First Book, and still more particularly (p. 311.), that from the phenomena and the things contained in that First Book, the demonstration was given of the elliptical orbits, the principle of gravitation, and the law of the inverse square of the distances, in the case of all planets and their satellites. It is manifest, therefore, that, without having seen the Principia, Leibnitz may have been so far enlightened by the view given of Newton's labours, as to be set upon applying the differential calculus to dynamical investigations, and to the motions of the heavenly bodies as the most important of all. He found, from the account of Newton's work, that he had succeeded in solving the great problem by mathematical investigation. He never had made any such attempt before; he now made it when he found Newton had successfully made it; and to a certain extent he himself succeeded.

A great difficulty arises in examining these propositions of Leibnitz and comparing them with Newton's, from the singular manner of using the letters in the diagrams and referring to them, as well as from the inaccurate printing. It however appears clearly enough that there are inconsistencies between different parts of his investigations; and Newton, when he breaks off with the words "Newtoniana tantum descripsit suo more, ac describendo nonnumquam-" after, in partly the same terms, having charged him with imitating fluxions and then erring in his imitation from not well understanding that method, appears to have intended making a similar remark upon his copying the dynamical propositions. (Rigaud, App. XIX.XX.) No doubt, if taken literally, and using the words centrifugal force in the sense in which Newton and indeed all others use it, there seems the greatest inaccuracy in the position that it varies inversely as the cube of the distance or of the radius vector, this being only true if the curve is a circle. But when we find that by conatus centrifugus he means what would be the centrifugal force in a circle 
whose radius is the radius vector at the given point of the curve, there is no error, and indeed he obtains his result in the same way as Newton does, nor could it well be obtained otherwise. His XVth proposition equally coincides with the equation to the centripetal force deduced from the VIth proposition of Book I. of the Principia; and the resolving the forces into two, one of which is in the direction of the radius vector, is according to that proposition.* By harmonical motion he means motion whereby equal curves are described by the radius vector in equal times; of which he gives no demonstration. Though we may be surprised with several other coincidences, it must be remembered that Leibnitz had the great benefit of Huygens' theorems on centrifugal forces; and if it be alleged that he threw his propositions into the form of dealing with centrifugal forces, the circumstance just adverted to will account for it without the suspicion that he did so to distinguish his investigations from those of the Principia. Still less have we a right to suggest that the attempt at reducing the whole within the scope of the hypothesis of vortices was made to conceal his knowledge of the Principia. It without doubt originated in the favour still entertained generally in that day for the Cartesian philosophy, of which not only Huygens was a zealous supporter $\dagger$, but Euler himself a disciple, half a century later. $\ddagger$

Upon the whole, we may affirm that the internal evidence is insufficient to support the charge. $\S$

* The exact agreement of his XVth prop. with our equation $\frac{d^{2} r}{d t}-\left.r \frac{\overline{d \theta}}{d t}\right|^{2}=$ centripetal force, $\theta$ being the angle of the radius vector with the axis, is to be noted among other coincidences.

† Letter to Leibnitz against the principle of gravitation, 1690 .

$\ddagger$ Mém. Acad. Paris, on the Tides, 1740 .

§ It should seem that M. Biot has, though with some hesitation, arrived at the same conclusion, from several passages of his most learned and valuable papers in the Journal des Savants, 1852; a paper which deserves to be 
diligently studied by all who take an interest in the history of the mathematical parts of the Newtonian philosophy. It was occasioned by the truly admirable publication of Mr. Eddleston (The Newton and Cotes Correspondence); and it throws important light upon some points that had not been sufficiently examined. No more profound admiration can be cherished for Newton than this eminent mathematician constantly and warmly expresses; nor can any disciple find the least reason to complain, unless, perhaps, he might dissent from the observation (p. 275.) on the inferiority of the Newtonian notation and supposition of generation compared with the Leibnitz plan, as if on the latter alone the calculus of partial differences could have been invented. The investigations from pp. 458. to 476., and from 522. to 535. are of great importance; they strikingly illustrate the facilities afforded by analytical process in the solution of dynamical problems; they, indeed, show how the propositions of Sections II. and III. (Lib. I.), are easily deduced from one general analytical formula, and may afford new ground for the supposition of those who think that Newton investigated algebraically, and demonstrated geometrically. M. Biot inclines strongly to the belief that the abstract of the Principia in the Acta Eruditorum, referred to in the text, was the work of Newton himself.

THE END. 





\section{DAY USE}

\section{RETURN TO DESK FROM WHICH BORROWED}

ASPOPMOMY, MATHEMATICS

AST.. STATISTICS LIBRARY

This publication is due on the LAST DATE stamped below.

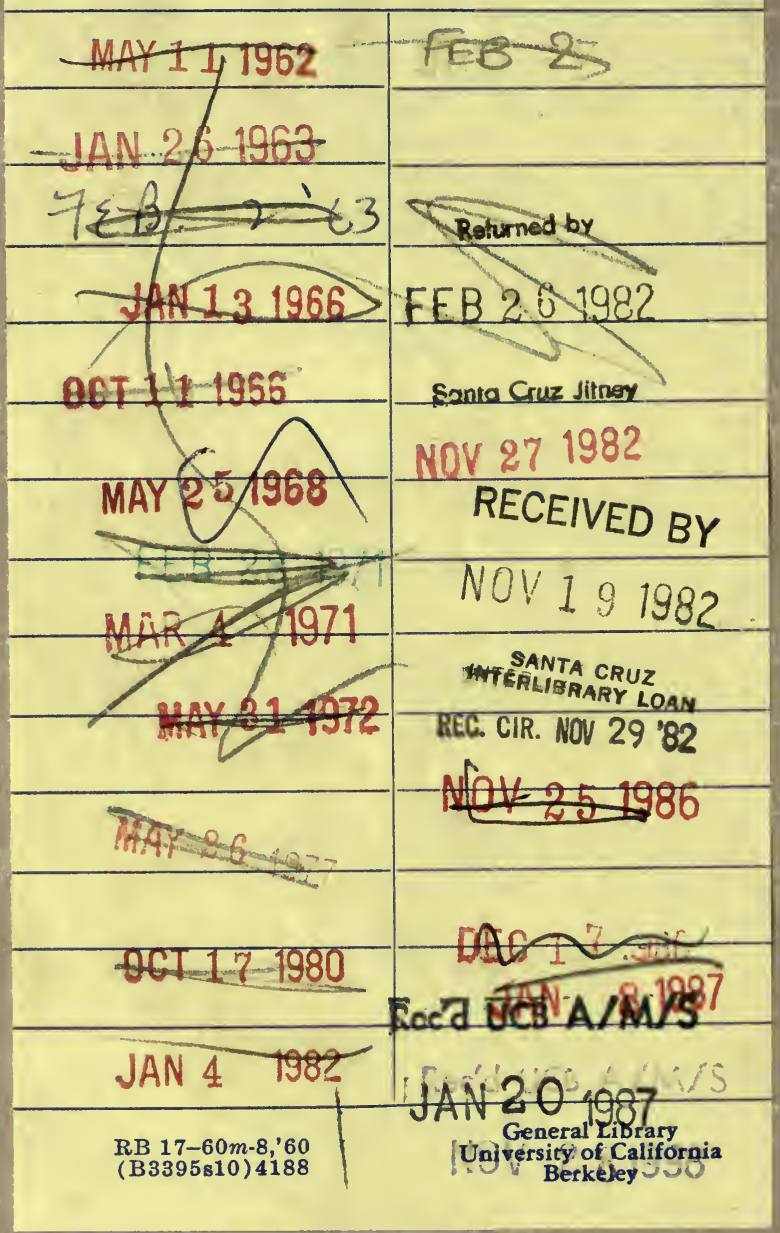



